
André Steiner

Komplexes Erzählen - Literatur auf 2+n-ter Stufe

Literaturtheorie | Band 3 
In Erinnerung an Lars Gustafsson (1936-2016)

André Steiner (Dr. phil.), geb. 1961, lehrt Literaturwissenschaft mit dem Schwerpunkt Neuere Deutsche Literatur an der Universität Bremen. Er ist Mitglied des Instituts für kulturelle Deutschlandstudien (ifkud) auf dem Bremer Campus. Seine Forschungsinteressen sind die Beziehungen zwischen Autor-Identität und literarischem Text, interkulturelle Literatur sowie Komplexität im Film. 
André Steiner

\section{Komplexes Erzählen - Literatur auf 2+n-ter Stufe}

Zu einer Theorie literarischer Komplexität 
Gefördert mit Mitteln der Staats- und Universitätsbibliothek Bremen

\section{Bibliografische Information der Deutschen Nationalbibliothek}

Die Deutsche Nationalbibliothek verzeichnet diese Publikation in der Deutschen Nationalbibliografie; detaillierte bibliografische Daten sind im Internet über http://dnb.d-nb.de abrufbar.

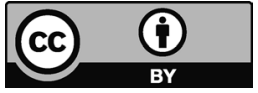

Dieses Werk ist lizenziert unter der Creative Commons Attribution 4.o Lizenz (BY). Diese Lizenz erlaubt unter Voraussetzung der Namensnennung des Urhebers die Bearbeitung, Vervielfältigung und Verbreitung des Materials in jedem Format oder Medium für beliebige Zwecke, auch kommerziell. (Lizenztext:

https://creativecommons.org/licenses/by/4.o/deed.de)

Die Bedingungen der Creative-Commons-Lizenz gelten nur für Originalmaterial. Die Wiederverwendung von Material aus anderen Quellen (gekennzeichnet mit Quellenangabe) wie z.B. Schaubilder, Abbildungen, Fotos und Textauszüge erfordert ggf. weitere Nutzungsgenehmigungen durch den jeweiligen Rechteinhaber.

\section{Erschienen 2021 im transcript Verlag, Bielefeld (C) André Steiner}

Umschlaggestaltung: Maria Arndt, Bielefeld

Umschlagabbildung: André Steiner, Wandbild im Bahnhof Voyageurs, Sousse/ Tunesien

Druck: Majuskel Medienproduktion GmbH, Wetzlar

Print-ISBN 978-3-8376-5849-1

PDF-ISBN 978-3-8394-5849-5

https://doi.org/10.14361/9783839458495

Buchreihen-ISSN: 2702-4458

Buchreihen-eISSN: 2703-0202

Gedruckt auf alterungsbeständigem Papier mit chlorfrei gebleichtem Zellstoff. Besuchen Sie uns im Internet: https://www.transcript-verlag.de Unsere aktuelle Vorschau finden Sie unter www.transcript-verlag.de/vorschau-download 


\section{Inhalt}

0. Komplexes Erzählen - Vorbemerkung $\ldots \ldots \ldots \ldots \ldots \ldots \ldots \ldots \ldots \ldots \ldots \ldots$

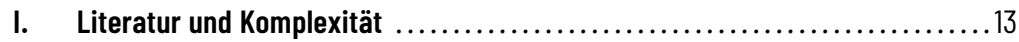

II. Komplexes Erzählen in Alban Nikolai Herbsts Roman

Buenos Aires. Anderswelt ...................................... 19

III. Aspekte der Rezeption komplexer Erzähltexte ..................... 33

IV. Vorläufige Bilanz - Gründe für das gegenwärtige Forschungsdefizit ....... 39

V. Der totale Roman als Vorläuferidee des komplexen Erzählens ............. 43

VI. Das Prinzip der alternativen Chronologien -

Komplexes Erzählen in Lars Gustafssons

Roman Herr Gustafsson persönlich ........................... 47

VII. Kybernetische Rückkopplung und Erinnerung

in Marcel Prousts Recherche ........................................ 59

VIII. Mehrstelligkeit und rückläufige Kausalität

als Modi der komplexen Erzählung

IX. Vom Umgang mit diskursiver Komplexität im Kontext emotionaler und kognitiver Partizipation an der komplexen Erzählung............... 75

X. Transkulturelles vs. hermeneutisches Modell des Verstehens unter Bedingungen der Komplexität 
XI. Narration in Echtzeit - Komplexes Erzählen in Navid Kermanis Roman Dein Name

XII. Inwiefern komplexes Erzählen zu einer veränderten Wahrnehmung der Lebenswirklichkeit von Lesern beitragen kann

XIII. Zum Problem der Übertragbarkeit von Begriffen aus der Theorie komplexer Systeme in den literaturwissenschaftlichen Diskurs

XIV. Das medial in sich eingeschlossene Subjekt -

Zum Verhältnis von Literatur und Leben unter Bedingungen der Echtzeit ... 123

XV. Zur Interaktion von Jetztzeit und geschichtlicher Vergangenheit Komplexes Erzählen in Alexander Kluges Lebensläufen

XVI. Erzählung und simulierte Wirklichkeit -

Komplexes Erzählen in Bodo Morshäusers Berliner Simulation

XVI. Genese von literarischer Bedeutung

durch strukturelle Selbstähnlichkeit

XVIII. Wende zum fraktalen Erzählen -

Reinhard Jirgls Roman Abschied von den Feinden

XIX. Fazit

XX. Quellenverzeichnis

XXI. Sachregister 


\title{
0. Komplexes Erzählen - Vorbemerkung
}

\author{
Der Geist der literarischen Erzählung ist \\ der Geist der Komplexität.
}

Peter Bieri

Das in der vorliegenden Studie behandelte Thema komplexes Erzählen ist neben der Verbindung zur Komplexitätsforschung auch vor dem Hintergrund eines verbreiteten Zeitgefühls zu verstehen. Es geht um das mitunter diffuse Gefühl eines globalen Zusammenhangs der Dinge, mit anderen Worten, um die Empfindung, dass zwar alles mit allem zusammenhängen mag, dass aber das Wissen fehlt, wie genau. Dass alles mit allem zusammenhängt, erfährt man in regelmäßigen Abständen aus den Medien in Wissenschafts-Talkshows wie SCOBEL (3sat) ${ }^{1}$ oder Radiosendungen wie EsSAY UND DISKURS (DLF), aber es ist nicht Teil unseres Erlebens in der Alltagswirklichkeit. Dort ist es eher so, dass das Eine mit dem Anderen nichts zu tun zu haben scheint. Beides scheint irgendwie wahr zu sein und für beides finde ich, wenn ich nur danach suche, Bestätigungen.

Auch hier kommt es offenbar darauf an, aus welcher Perspektive die Dinge betrachtet werden. Ob dies aus der Sicht des einzelnen Subjekts oder des intellektuellen Diskurses geschieht, macht einen großen Unterschied. Auf der Ebene des Diskurses gerinnt die Formel des »alles hängt mit allem zusammen« spätestens seit den 9oer Jahren zur Prämisse jedes Nachdenkens über komplexe Zusammenhänge, die diskursübergreifend und in globalem Maßstab inzwischen zum Gegenstand eines neuen Diskurses, der Komplexitätsforschung, geworden sind. Auf der Ebene subjektiven Erlebens manifestiert sich hingegen das genaue Gegenteil des postulierten, allumfassenden Zusammenhangs: Ich habe gerade kein Bild mehr vom Ganzen. Postulierte Zusam- 
menhänge sind nicht mehr wirklich wahr, sondern nur noch möglich, bzw. haben den Status von Prognosen. ${ }^{2}$

Dass alles mit allem zusammenhängen soll, kann ich für mich selbst folglich nicht verifizieren, solange das Bezugssystem mein subjektives Erleben von Wirklichkeit ist. ${ }^{3} \mathrm{Zu}$ einer solchen allumfassenden, ganzheitlichen, globalen Realität habe ich, abgesehen von den Hiobsbotschaften der Medien, gar keinen oder eben höchstens virtuellen Kontakt. Meine eigene, medienunabhängige Erfahrung von Wirklichkeit sagt mir hingegen, dass das eine mit dem anderen oft genug gar nichts zu tun hat, dass man mitunter in Wissenschaft und Technik Lösungen für winzige Einzelprobleme findet, die aber auf das große Ganze keinerlei Auswirkungen haben und untereinander nicht anschlussfähig sind. Mathias Greffrath hat dieses Lebensgefühl mit Olga Tokarczuk in einem Radioessay wie folgt auf den Punkt gebracht:

»Wir beginnen die Welt nur noch in Stückchen zu sehen, alle getrennt voneinander, in kleine Teilchen, getrennt voneinander, und die Wirklichkeit in

2 So spricht auch der Philosoph Markus Gabriel davon, dass es einen solchen Cesamtzusammenhang schlicht nicht gibt, weder als Gesamtheit der Tatsachen (metaphysisch) noch als Cesamtheit der Dinge, aus denen die Welt als ganze bestünde. Trocken konstatiert er: »Es hängt schlicht nicht alles mit allem zusammen; es gibt keinen Allzusammenhang [...] «. Markus Gabriel: Fiktionen, Berlin: Suhrkamp 2020, S. 36 f. Gegenstände, auch fiktionale, existieren bei ihm in sog. Sinnfeldern, die anthropologisch gedacht von der menschlichen Einbildungskraft eigens erzeugt werden.

3 Ist im Folgenden von subjektiv, Subjekt bzw. Subjektivität die Rede, so ist damit der Bedeutung nach gemeint (1) Personalität als verkörperte und moralisch zurechenbare Subjektivität sowie (2) Individualität als die Fähigkeit von Subjekten, eine unverwechselbar singuläre Biografie und (3) ein eigenes Urteil, eine eigene Sichtweise, einen eigenen Ceschmack zu haben. Vgl. ex negativo Manfred Frank: Ansichten der Subjektivität, Berlin: Suhrkamp 2012, S. 353. Es schließt sich daran, wie sich zeigen wird, ein Sprechen über Sichtweisen an, die perspektivisch different sein können, wie etwa die zwischen der ersten und der dritten Person Singular, welche in der natürlichen Sprache - wie auch in literarischen Texten - pronominal unterschiedlich markiert sind. Wendet sich die aus einer solchen Perspektive gemachte Beobachtung auf sich selbst zurück, so entsteht daraus eine Verdoppelung der subjektiven Instanz, die aus der propositionalen Zweistelligkeit (jeder Beobachtung) von Beobachter (Subjekt) und Beobachtetem (Objekt) resultiert. Diese Verdoppelung wird als innere Wahrnehmung von Lesern, analog zur Vorstellung als Produkt der Einbildungskraft, eine wichtige Rolle bei der Bewertung eigener Lektüreerfahrungen und daraus für das eigene Leben zu ziehenden Schlüssen/zu fällenden Entscheidungen spielen. 
der wir leben, bestätigt dies Gefühl: Ärzte behandeln uns nach Fachgebieten, die Steuern, die wir zahlen haben nichts zu tun mit dem Pflug, der den Schnee räumt auf der Straße zur Arbeit, unser Mittagessen hat nichts zu tun mit der industriellen Farm, und mein neues Top mit einer schäbigen Fabrik irgendwo in Asien. Alles ist getrennt, alles lebt getrennt, ohne Verbindung.< Olga Tokarczuk drückt ein verbreitetes Lebensgefühl aus: Alles dringt auf mich ein, aber ich habe kein Bild, kein Gefühl für das Ganze mehr und nichts kann ich ändern. $\ll^{4}$

Beschrieben wird eine Welt aus Partialobjekten, die kein Ganzes mehr ergibt, weil sie sich grenzenlos erweitern lässt und genau so erlebt wird. Eine Welt, in der nicht nur die Gegenstände ohne Bezug auf einen gemeinsamen Sinnhorizont, verdinglicht und abgetrennt, ähnlich wie in der metaphysischen Malerei Giorgio de Chiricos, sondern auch das menschliche Leben selbst jede humane Implikation zu verlieren droht. Diese Koinzidenz kann als sinnfälliger Ausdruck für die Überforderung des Einzelnen durch Phänomene des Komplexen verstanden werden, für deren allerdings nur vorläufige, theoretische Bemeisterung selbst die Wissenschaft nur durch kooperative Verknüpfung verschiedener Diskurse und Disziplinen in der Lage ist.

Wenn ich andersherum die unterstellten Zusammenhänge nicht verstehe, werde ich auch die Frage nach dem Warum meines Handelns in einer zuweilen überkomplex gewordenen und deshalb fragmentiert erlebten Lebensrealität kaum beantworten können. Ein solches Nicht-Verstehen ist regelmäßig verbunden mit der Unfähigkeit zur Versprachlichung des damit zusammenhängenden Erlebens. Wenn ich keine Sprache dafür habe, was mit mir vorgeht, weil die Umstände und größeren Zusammenhänge, in denen dies geschieht, undurchschaubar werden, bin ich den blinden Kräften des Zufalls ausgeliefert. Und es wäre nicht übertrieben, angesichts einer solchen Überforderung durch Komplexität von einer zeitgenössischen Variante symbolischer Dekapitation zu sprechen, die in der deutschen Sprache auch mit der verharmlosend euphemistischen Wendung, jemand habe seinen Kopf verloren, zum Ausdruck gebracht werden kann. Aus diesem Grund wird mit erneuter Dringlichkeit nach Sinn und Bedeutung singulärer, subjektiver Identität innerhalb einer hochgradig vernetzten, pluralisierten, in weiten 
Bereichen globalisierten Lebenswelt gefragt. Freilich vor dem Hintergrund des Problems, auf welche Weise komplexes Erzählen dem subjektiven Bedürfnis von Lesern (das allzu oft Ausdruck objektiver (Zwangsverhältnisse sein kann) nach Veränderung eigener Befindlichkeit oder Erweiterung des eigenen Horizonts innerhalb unverstandener Lebenslagen gerecht zu werden vermag.

Wendet man sich im gegebenen Zusammenhang der Literatur zu, so mag es sich im Grunde fast von selbst verstehen, dass, sobald die Weltverhältnisse komplexer werden, die Literatur ihrerseits komplexer werden muss, will sie die gewordenen Verhältnisse angemessen reflektieren. Doch ist damit noch keine Antwort auf die Frage nach den je spezifischen Manifestationen von Komplexität im einzelnen Werk gegeben. Mit anderen Worten, nach der Sprache bzw. den erzählerischen Mitteln, die für die oben formulierte Erfahrung gefunden werden. Denn diese können als eigentlicher Gegenstand wissenschaftlicher Neugier der vorliegenden Arbeit betrachtet werden. Des Weiteren liegt es nahe, dass doch eigentlich jedes literarische Schreiben, auch wenn es nicht so komplex sein mag, wie die im Folgenden $\mathrm{zu}$ besprechenden Romane, schon durch den Leser, der in einer vielfältiger gewordenen Wirklichkeit lebt, mit mehr Bedeutungsmöglichkeiten aufgeladen wird, als noch zuvor der Fall. Daher sucht der im weiteren Verlauf zu entfaltende, diskursive Ansatz, anders als Gérard Genette bei seinem Entwurf einer Literatur auf zweiter Stufe, ${ }^{5}$ nach Relationen, die den literarischen Text und seine integralen Figurationen von Form und Inhalt in Richtung seiner Genese aufseiten des Autors wie des Rezipienten erweitern. Diese beiden Positionen innerhalb des umfassenderen Systems literarischer Kommunikation werden dabei als Subsysteme begriffen, deren Komplexität ihrerseits berechtigt, von verschiedenen Stufen/Ebenen zu sprechen, die als einzelne genommen, wie sich zeigen wird, selbst in den Bereich kritischer Komplexität hineinreichen.

So ist der im Titel genannte Zusatz >Literatur auf zwei plus n-ter Stufe als Hinweis auf Genettes Konzept der Hypertextualität gemeint, bei der sich Motive und Strukturen eines gegebenen literarischen Textes zumeist diachron mit ihren historisch früheren Manifestationen intertextuell überlagern. Im Unterschied zu Genettes Betrachtungsweise, die zwar auch von einer fortwährenden Zirkulation der Texte ausgeht, bei der die alten Werke ständig 
in einen neuen Sinnkreislauf eingespeist werden, ${ }^{6}$ verschiebt die vorliegende Untersuchung den Fokus hin zu den Prozeduren der Erzeugung von Sinn und Bedeutung, beobachtet aus den Perspektiven des Schreibenden wie des teilnehmenden Lesers. Dabei wird zunächst zwar auch auf das Niveau der literarischen Diegese mit den dazugehörigen inhaltlichen und formalen Bestimmungen rekurriert. Anschließend wird diese Stufe jedoch in Richtung der beiden genannten Positionen überschritten, die sich allerdings, in je eigener Weise, als rekursiv, d.h. instantan mit dem Schreibprojekt/der Lektürevorlage, rückgekoppelte erweisen. ${ }^{7}$

6 Vgl. ebd., S. $534 \mathrm{f}$.

7 Das Stufenmodell wird noch um eine Ebene komplexer, sobald die Differenz zwischen den Lesarten individueller Leser mit berücksichtigt wird. Dazu schreibt Vilém Flusser: »Der Text ist bedeutungsıvolk, und diese Fülle wird erst von jedem seiner Leser auf eigene Weise ausgebeutet (gedeutet). Ein Text ist umso bedeutungsvoller, je größer die Zahl seiner Lesarten. «Vilém Flusser: Die Schrift. Hat Schreiben Zukunft?, Frankfurt a.M.: Fischer 1992, S. 36. 



\section{Literatur und Komplexität}

Mit Blick auf die Strukturen des Erzählens, die bisher in der Forschung selbstreferenziell oder auch selbstreflexiv genannt wurden, ${ }^{1}$ lässt sich bei einer Erweiterung der Perspektive auch vom komplexen Erzählen sprechen. Ein Begriff, der von Dietmar Mieth im Zusammenhang der narrativen Ethik gebraucht wurde und hier in den Kontext von systemischer Komplexität und literarischem Erzählen eingeführt werden soll. ${ }^{2}$ Komplex bedeutet dann nämlich, dass nicht nur die Bedingungen des Erzählaktes selbst, sein Zustandekommen, seine ursächlichen Begründungszusammenhänge und sein Prozesscharakter im literarischen Text mit reflektiert werden, ${ }^{3}$ also das, was man mit

Zur Definition des Begriffs vgl. Dorea Dauner: Literarische Selbstreflexivität. Dissertation, Universität Stuttgart 2009. Online einsehbar unter $h t t p: / / d x$.doi.org/10.18419/op us-5332 vom 19.05.20. Sie unterscheidet in ihrer Arbeit zwischen produktions- und rezeptionsorientierter Selbstreflexivität. Zum Verhältnis der beiden Terme selbstreferenziell und selbstreflexiv vgl. besonders das Kapitel »1.1. Vorbemerkungen«, in: Michael Scheffel: Formen selbstreflexiven Erzählens. Eine Typologie und sechs exemplarische Analysen, Tübingen: Niemeyer 1997, S. 9 f. Selbstreferenziell stammt aus dem Diskurskontext des Radikalen Konstruktivismus der 9oer Jahre und bezieht sich dort auf die kognitiv-neuronale Organisationsform des Cehirns als autopoietischem System. Der Begriff ist dann u. a. von der Literaturwissenschaft als Synonym für selbstreflexiv adaptiert worden.

2 Vgl. zur Definition des komplexen Erzählens Dietmar Mieth: Moral und Erfahrung I. Grundlagen einer theologisch-ethischen Hermeneutik, 4. überarb. und ergänz. Neuaufl., Fribourg: Universitätsverlag 1999, S. 102-105.

3 Cemeint ist damit, dass wie in E.T.A. Hoffmans Serapions-Brüder nicht nur die Erzählung oder das Märchen als solches präsentiert werden, sondern der Leser durch die Beschreibung der Umstände, die zur Narration geführt haben, Wichtiges über den Kontext des Erzählaktes erfährt. Dies geschieht durch die Einbettung in einen erzählerischen Rahmen, aus dem jeweils hervorgeht, wer aus der Gemeinschaft der versammelten Erzähler nun mit dem Vortrag an der Reihe ist. Auch hier handelt es sich gegenüber einfachen Formen des Erzählens um eine Zunahme von Komplexität, denn 
einem Begriff aus der Chaostheorie seine Randbedingungen nennen könnte, sondern dass zudem mit mehr Emphase auf eine grundlegende Eigenschaft literarischen Erzählens verwiesen wird: Seine Fähigkeit nämlich narrativ auch dort noch Zusammenhänge herstellen zu können, wo alle anderen Modelle der Welterklärung statt Kohärenz und Kontinuität nur Differenz und Diskontinuität, sprich Zusammenhanglosigkeit und Nicht-Identität, erkennen können. Dies alles freilich unter Berücksichtigung noch zu erörternder Aspekte der Rezeption, die bei der kreativen Aneignung literarischer Texte wirksam werden. ${ }^{4}$

So bleibt, bis auf Weiteres, dem Erzählen vorbehalten, wonach man besonders in der Physik oder neuerdings auch der Soziologie vergeblich gesucht hat: Die Vereinheitlichung mehrerer Teilsysteme, resp. Teiltheorien zum Modell einer Ganzheit von Welt/Wirklichkeit bzw. Gesellschaft oder zumindest das Vermögen, Teilbereiche, resp. Teilerzählungen so miteinander zu verknüpfen, dass die Übergänge plausibel anschlussfähig sind für immer weitere Fortsetzungsgeschichten einer einmal begonnenen, pluralisierten Form der Theorie wie der Narration.

Dass es schon seit längerer Zeit so etwas wie eine Herausforderung durch Komplexität in vielen Bereichen des Wissens und der Gesellschaft gibt, davon zeugt die Entwicklung eines eigenen Diskurses mit der Bezeichnung Komplexitätsforschung. ${ }^{5}$ Hier nun lässt sich eine Verbindung zum Erzählen über den für alle komplexen Phänomene geltenden »Verzicht[...] auf eine zentrale Instanz ${ }^{6}$ herstellen. Dass eine solche zentrale Funktionsstelle weder in modernen Gesellschaften noch in komplexen neuronalen, ökonomischen oder

ähnlich wie in den Wissensbereichen, von denen noch die Rede sein wird, werden nunmehr die Randbedingungen, die zur Entstehung eines Phänomens - hier der Narration - beitragen, in die Gesamtdarstellung mit einbezogen. Vgl. dazu auch meinen Beitrag »Zyprian oder Zombie?«, in: Norman Kasper/Gert Theile (Hg.), Asozialität und Aura. Wolfgang Hilbig und die Romantik, Leiden et al.: Fink 2017, S. 78 f.

4 So lässt sich die zuvor benannte Fähigkeit der Literatur zur Fusion verschiedenartigster Realitäten neuerdings in der Blending-Theorie auch damit erklären, dass im Prozess der Hybridisierung von mindestens zwei mentalen Repräsentationen, die sich sogar widersprechen können, neue, emergente Bedeutungen resultieren. VgI. dazu Kap. IX.

5 Einer der wichtigsten Vertreter ist sicher der mittlerweile emeritierte Philosoph und Wissenschaftstheoretiker Klaus Mainzer. Einen Überblick über die verschiedenen Forschungsansätze gibt er in seinem UTB-Band Klaus Mainzer: Komplexität, Paderborn: W. Fink 2008.

6 Armin Nassehi: Die letzte Stunde der Wahrheit. Kritik der komplexitätsvergessenen Vernunft, Hamburg: Sven Murmann 2017, S. 89. 
Klimamodellen besetzt ist, hat dazu geführt, dass sich die Zusammenhänge, die zur Erklärung dieser Phänomene bemüht werden, nicht mehr einfach linear darstellen, sprich erzählen lassen. Gebraucht werden dazu komplizierte Verfahren aus der statistischen Informatik, der Thermodynamik und der Chaostheorie, die jeweils nach unterschiedlichen Logiken funktionieren und aus diesem Grund innerhalb einer ganzheitlichen Vorstellung von Welt/Wirklichkeit oder Gesellschaft nicht miteinander kompatibel sind. Man braucht jedoch diese Instrumente, um das Wie der Prozesse zu erklären, weil es - ähnlich wie in der Erzählforschung, wo man aus diesem Grund die Instanz des Erzählers eingeführt hat - nicht ausreicht zu sagen, das Gemeinte geschehe einfach von selbst.

Denkt man über die Möglichkeit einer Analogisierung zwischen dem für komplexe Systeme charakteristischen Verlust der zentralen Instanz und bestimmten Mustern literarischen Erzählens nach, so sind es neben den Strukturen, die Dieter Wrobel in seiner Studie über Analogien zwischen Chaostheorie und literarischem Text untersucht hat, ${ }^{7}$ zunächst zwei grundlegende Erzählformen, die dafür in Betracht kommen. Gemeint ist einmal das polyphone (vielstimmige) Erzählen wie etwa im Roman Mutmaßungen über Jakob (1959) von Uwe Johnson, bei dem sich mehrere Erzählerstimmen in der Rekonstruktion des Romangeschehens gegenüberstehen. ${ }^{8}$ Dann ein Erzählen, das durch die Heterogenität der erzählten Welt(en) auf die Komplexität der modernen Gesellschaft antwortet, indem auch im Roman »[d]ie unterschiedlichen Logiken, Intelligenzen und Funktionen «, wie Armin Nassehi mit Bezug auf die gesellschaftlichen Verhältnisse schreibt, »sich wechselseitig je nach eigenen Verarbeitungsregeln wahr[nehmen] und [...] dadurch ein System [erzeugen], das nicht aus einem Guss ist. «"

Gekoppelt an Nassehis Theorem für Komplexität, dass etwas, hier der literarische Text, gedacht in seiner Komplexion umfassender Darstellung von (fiktionaler) Wirklichkeit und kognitiven Prozessen der Rezeption wie

7 Dieter Wrobel: Postmodernes Chaos - Chaotische Postmoderne. Eine Studie zu Analogien zwischen Chaostheorie und deutschsprachiger Prosa der Postmoderne, Bielefeld: Aisthesis 1997. Ich gehe auf typische Strukturen komplexer Systeme wie etwa Fraktale, Bifurkation oder den Schmetterlingseffekt im literarischen Text hier noch nicht ein. Dieter Wrobel hat dies in seiner Studie am Beispiel von Sebald, Modick und Krüger getan. Es soll hier zunächst nur um die Instanz des Erzählers gehen. Vgl. Wolfgang Emmerich: Kleine Literaturgeschichte der DDR, erweiterte Neuausgabe, Berlin: Aufbau 2000, S. 151.

9 A. Nassehi: Letzte Stunde der Wahrheit, S. 120. 
der Produktion, simultan unterschiedliche (Bedeutungs-)Zustände annehmen kann, mithin ein Selbes sich unterschiedlich beschreiben lässt (Mehrfachkodierung), ${ }^{10}$ ergibt sich zudem aus einem solchen Design, dass die literaturwissenschaftliche Analyse nicht beim Text und seinen Binnenstrukturen stehen bleiben kann, sondern seine kognitiv-systemischen Voraussetzungen aufseiten des Autors sowie seine verhaltensrelevanten Wirkungen beim Leser ${ }^{11}$ - verstanden jeweils in ihrer Prozessualität - mit berücksichtigen muss. So kommt es, dass im Anschluss an die Erörterungen des vorliegenden Abschnitts, ausgehend von Mieths Ansatz zum komplexen Erzählen, der Möglichkeit einer neuen lebensweltlichen Orientierung durch die Rezeption komplexer Erzähltexte das Wort geredet wird. Weil es dabei $\mathrm{zu}$ systemischen Überschreitungen zwischen Text und - hier besonders im Fokus - der empirischen Wirklichkeit des Lesers kommt, ist der Subjektbegriff, der dabei zugrunde gelegt wird, derjenige einer empathischen Projektion realer menschlicher Subjektivität in die Figuren der miteinander verschalteten Ebenen der zu untersuchenden Erzähltexte von Alban Nikolai Herbst bis Reinhard Jirgl.

Unterstellt wird dabei, dass es auf der Seite der Produktion analoge Prozesse gibt, die mit der Übertragung der Subjektivität des Autors in seinen Text zusammenhängen. Fraglich ist nun, ob eine solche mimetische Übertragung, wie sie als Phänomen auch im Roman Buenos Aires. Anderswelt von Herbst zwischen dem Ich-Erzähler und einer der erzählten Figuren stattfindet, unter der Voraussetzung der operationellen Geschlossenheit bedeutungsgenerierender kognitiver Systeme nicht mindestens problematisch wird und eigent-

\footnotetext{
10 Vgl. ebd.

11 Wenn i.F. auf den Leser Bezug genommen wird, so versteht sich dies im Sinne einer »Teilnahmehandlung[...] an Literarischer Kommunikation«, wie sie in der ETL (Empirische Theorie der Literatur) von Siegfried J. Schmidt vorgeschlagen wird. Siegfried ] Schmidt: Grundriß der Empirischen Literaturwissenschaft, Frankfurt a.M.: Suhrkamp 1991, S. 167. Obgleich davon auszugehen ist, dass die Geschlechtszugehörigkeit empirischer Subjekte, die im System literarischer Kommunikation die Position des Lesers einnehmen, in je spezifischer Weise zu einem uneinheitlichen Rezeptionsverhalten führt, ist dieser Aspekt nicht Gegenstand der vorliegenden Untersuchung und bleibt daher außer Betracht. Ein solcher an der Cendertheorie orientierter Ansatz müsste an anderem Ort eingehender behandelt werden. Da es sich hier um eine eher systemische Betrachtungsweise handelt, wird entsprechend auch auf Markierungen des sozialen Geschlechts wie Gender Gap oder Gender-Sternchen im Kontext der Rezeption verzichtet und immer nur vom Leser im generischen Maskulinum die Rede sein.
} 
lich durch ein neues Paradigma ersetzt werden müsste. Geprüft wird deswegen die Annahme, dass die eigentümliche Potenzialität literarischer Texte, die in komplexer Weise erzählen, wohl gerade in der rückwirkenden Austauschbeziehung zwischen ihren systemischen Komponenten (Autor - Text - Leser) besteht, aus der dann Sinn/Bedeutung erst hervorgeht und die auch als Emergenz begriffen werden kann. ${ }^{12}$ Dabei werden Wolfgang Isers Überlegungen zum Paradigma der Emergenz mit ihren verschiedenen Modalitäten in den Textanalysen zugrunde gelegt, um auf diese Weise die besondere Form der Interaktion zwischen Leser und komplexer Erzählung angemessen beschreiben zu können.

Zudem werden Aspekte aus der neurowissenschaftlichen Kreativitätsforschung (Linke) in die Argumentation einfließen, nicht nur um Mieths Thesen für den vorliegenden Zusammenhang zu plausibilisieren, sondern auch, um in komplexitätstheoretischer Hinsicht die Möglichkeit multipler Sichtweisen auf den gleichen Text thematisch angemessen zu explizieren. Im Verlauf der weiteren Arbeit wird es nicht nur darum gehen, nach Berührungspunkten zwischen den verschiedenen Diskursen zu suchen, die gemeinsam zum Verständnis des komplexen Erzählens beitragen können. Darüber hinaus sollen die unterschiedlichen methodischen Ansätze aus der Komplexitätsforschung (Mainzer), der Soziologie (Nassehi), der Neuroethik (Linke), der Rezeptionsästhetik (Iser), der kognitiven Literaturwissenschaft (Schmidt, Hiergeist, Wege, Scheffer) sowie der Neuro-, Subjekt- und Medienphilosophie (Varela, Virilio, Baudrillard, Flusser), wo möglich, in einen interdisziplinären Dialog gebracht werden, um auf diese Weise über die Grenzen der einzelnen Diskurse hinweg einen Beitrag zur Theorie literarischer Komplexität leisten zu können. Dabei wird sich zeigen, dass es so etwas wie letzte Lösungen nicht geben kann, dass vielmehr die Wissensakteure aktiv mit der Perspektivendifferenz der beteiligten Diskurse werden umgehen lernen müssen. ${ }^{13}$

12 Vgl. dazu den Aufsatz von Siegfried ]. Schmidt.: »Über die Rolle von Selbstorganisation beim Sprachverstehen«, in: Wolfgang Krohn/Cünter Küppers (Hg.), Emergenz: Die Entstehung von Ordnung, Organisation und Bedeutung, Frankfurt a.M.: Suhrkamp 1992, S. 293-333. Zum Problem der operationellen Ceschlossenheit kognitiver Systeme siehe im gleichen Band die Ausführungen von Gerhard Roth:»Entstehung von Bedeutung im Cehirn«. Vgl. dazu mit Bezug auf die Perspektivendifferenz in der Cesellschaft A. Nassehi: Letzte Stunde der Wahrheit, S. 200. 



\section{Komplexes Erzählen in Alban Nikolai Herbsts Roman Buenos Aires. Anderswelt}

Sucht man in der deutschsprachigen Gegenwartsliteratur ein Beispiel für die im ersten Abschnitt mit Rekurs auf Nassehi gegebene Beschreibung einer heterogenen Erzählwelt, so findet sich eine solche Konstellation im Roman Buenos Aires. Anderswelt von Alban Nikolai Herbst. ${ }^{1}$ Dort ist in der Modellwelt Garrafff ein Team der Firma Cybergen - neben Alban Herbst, ${ }^{2}$ die Figuren Sabine Zeuner und Herbert Lerche - mit der Programmierung simulierter Welten beschäftigt. Aus Sicht jeder einzelnen dieser Welten ist nicht entscheidbar, ob

1 Es handelt sich um den zweiten Teil der Anderswelt-Trilogie, die mit Thetis. Anderswelt (1998) beginnt und mit Argo. Anderswelt (2013) endet. Intertextuell steht die RomanTrilogie innerhalb der Fantastik mit den Neuromancer-Romanen William Gibsons in Zusammenhang.

2 Hier sei angemerkt, dass die Herstellung eindeutiger Differenz in Bezug auf die Identität des Signifikats der Zeichenkette >Alban/Nikolai/Herbst bzw. seiner Variationen vom Autor bewusst subvertiert wird. Es kommt auf diese Weise zu einer Multiplizität des Autornamens, die vor dem Hintergrund postmoderner Ästhetik zu verstehen ist, welche - mit Baudrillard gesprochen - den simulativen Charakter gegenwärtiger Wirklichkeitsproduktion und -rezeption mit berücksichtigt. Vgl. dazu Christoph Jürgensen: »Ins Netz gegangen - Inszenierungen von Autorschaft im Internet am Beispiel von Rainald Goetz und Alban Nikolai Herbst«, in: Christoph Jürgensen/Gerhard Kaiser (Hg.), Schriftstellerische Inszenierungspraktiken - Typologie und Geschichte, Heidelberg: Winter 2011, S. 403-422, hier S. 417 unten. Die mehrfache Zuschreibbarkeit des Autornamens steigert sich innerhalb einer Poetik der Komplexität noch dadurch, dass sie nicht auf die genannten Romane beschränkt bleibt, sondern gerade in der Interrelationalität, also den medienübergreifenden Wechselbeziehungen zwischen den webbasierten, schriftlichen Produktionen Herbsts (z.B. das Weblog Die Dschungel. Anderswelt) und den herkömmlich in Buchform erschienenen, erzählerischen Texten zum Ausdruck kommt. Vgl. ebd., S. 416 f. 
es sich um eine programmierte, also informatisch erzeugte oder um eine natürliche Welt handelt. Im Folgenden soll es daher um die Frage gehen, wie sich der Übergang bzw. Wechsel zwischen den Welten im Erleben des Subjekts spiegelt. Damit ist insbesondere die Relation zwischen dem erzählenden Programmierer Alban Herbst und seinem »kybernetischen Nachstellmodell ${ }^{3}$ Hans Erich Deters gemeint, wo mitunter übergangslos, also ohne Absatz oder Inquit-Formel, von einem Satz zum nächsten die Referenz des erzählenden Subjekts, d.h. die Identität des Erzählers, wechselt. ${ }^{4}$ Ein Beispiel dafür findet

Alban Nikolai Herbst: Buenos Aires. Anderswelt, Berlin: Berlin Verlag 2001, S. 179. Wie bereits Innokentij Kreknin ausgeführt hat, lässt sich Hans Erich Deters als Spaltfigur des Autors Alban Nikolai Herbst deuten. In diesem Sinn hat sich jedenfalls Herbst selbst in dem Aufsatz Eine Beichte: Wie ich zum Schreiben kam (2002) geäußert. Neben diesem Text stützt sich Kreknin bei der Herleitung der verwickelten Selbst-Poetik von Herbst auf dessen Grimmelshausen-Preisrede (1995), verliehen für Wolpertinger oder Das Blau (1993), wo bereits davon die Rede ist, dass zwischen dem empirischen Ich des Alban Nikolai Herbst und dem Autor gleichen Namens rigide getrennt werden müsse. Die dort gegebene Begründung, dass der Autor als ein anderer zum empirischen Ich hinzutrete und entsprechend in der dritten Person Sg. angesprochen werden müsse, wird dann noch einmal iteriert, indem Deters, der als Figur dem Roman Die Verwirrung des Cemüts (1983) entspringt und als Hauptfigur in Wolpertinger wieder auftritt, laut Herbst mit ihm selbst einen Pakt über die Weitergabe der Autorfunktion geschlossen hätte: »Denn was ich zu meinen Texten beigetragen habe, ist lediglich mein Name. Dafür hat Hans Deters mich bezahlt und bezahlt mich noch."So Herbst in Eine Beichte. Zit. nach Innokentij Kreknin: Poetiken des Selbst. Identität, Autorschaft und Autofiktion am Beispiel von Rainald Coetz, Joachim Lottmann und Alban Nikolai Herbst, Berlin et al.: De Gruyter 2014, S. 371. Demnach wäre Deters der wirkliche Autor der literarischen Fiktionen Herbsts, für die er sozusagen vertraglich den eigenen Namen an Deters abgetreten hätte. Vgl. für den Zusammenhang insgesamt ebd., S. $370 \mathrm{f}$.

Neben diesen beiden Formen sind freilich noch andere Spielarten komplexen Erzählens denkbar. Den Versuch einer allgemeinen Definition macht Dietmar Mieth: »Unter komplexem Erzählen verstehe ich Formen des Erzählens, die die komplexe Erfahrung der Lebenswirklichkeit widerzuspiegeln versuchen. Formen des komplexen Erzählens entstehen z. B., wenn sich verschiedene Handlungsstränge miteinander kreuzen, wenn die Zeitebenen des Ceschehens sich verschieben, wenn die Perspektiven der Personen ständig wechseln, wenn Analysen und Reflexionen miterzählt werden." D. Mieth, Moral und Erfahrung, S. 102. Mieth bezieht sich im Folgenden auf die Josephromane Thomas Manns als Versuch der Übertragung und Neuanpassung einer ursprünglich biblischen Ceschichte auf die Verhältnisse des gegenwärtigen, komplexer gewordenen Bewusstseins sowie auf Musils Mann ohne Eigenschaften, in dem sich Ratio und Cefühl auf komplexe Weise begegnen. Vgl. ebd., S. 103 f. Dass komplexes Erzählen nicht zwingend mit der Großform Roman verbunden ist, hat Rodiek am Bei- 
sich im Kapitel ... die Unendlichkeit, wo Deters nach einem Ausflug in der labyrinthischen Simulation Garrafff (W3) mithilfe eines abgespulten Fadens in seine Wohnung in der Berliner Dunckerstraße zurückkehrt:

»Endlich stand er auf, benommener noch, als mich zuvor die Halluzinationen gemacht hatten, und stieg langsam und nachdenklich die Treppen nach oben. Das Schild war noch da. Herbst \& Deters Fiktionäre. Ich versuchte, es mit den Fingern abzuziehen; es war nicht verschraubt, nur geleimt, aber mit Sekundenkleber oder sowas, denn auch, als ich es mit einem Schraubenzieher abhebeln wollte, den ich zwischen Blechkante und Türholz stemmte, löste sich das widerliche Ding um keinen Viertelmillimeter. Vor Anstrengung fing Deters zu schwitzen an [...]. $\ll^{5}$

Die zitierte Passage ist nur eines von vielen Beispielen dafür, ${ }^{6}$ dass es unter Bedingungen fortgeschrittener Komplexität im Roman zu einer Verschmelzung der Ebenen von sogenannter realer und simulierter Wirklichkeit kommt, die sich sprachlich im übergangslosen Wechsel der Perspektive von der 3. in die 1. Person Singular und wieder zurück manifestiert. Das Er (Deters) und das Ich (Herbst) im Textausschnitt befinden sich erlebnismäßig dann zwar auf der gleichen Ebene, ${ }^{7}$ repräsentieren aber Identitäten, die auf ontologisch verschiedenen Ebenen angesiedelt sind: Der Avatar im System und sein Programmierer außerhalb davon in der intradiegetisch scheinbar realen Welt sind sowohl logisch als auch hierarchisch voneinander unterscheidbar. So sollte es zumindest sein. ${ }^{8}$ Was eigentlich aufgrund der Hierarchie zwischen den beiden Realitäten ausgeschlossen ist, passiert eben

spiel der Kurzgeschichte Bifurcaciones des spanischen Erzählers José Maria Merino gezeigt. Vgl. Christoph Rodiek: Untersuchungen zur spanischen Kurzgeschichte der Cegenwart (1980-2010). Definitionen, Analysen, Fallstudien, Frankfurt a.M.: Peter Lang 2012, S. 213-226.

5 A.N. Herbst: Buenos Aires, S. 176 f.

6 Weitere Beispiele finden sich ebd., S. 169 und 175.

7 Anders als ich räumt Bobzin zwar ein, dass es sich um einen Sprecherwechsel handelt, meint aber, dass beide Personalpronomen sich zugleich auf Deters bezögen im Sinne des Verweises »auf verschiedene Möglichkeiten und vielleicht auch verschiedene Welten. « Henning Bobzin: Von Bremen in die Anderswelt. Über Identität und Realität in Prosahauptwerk, Poetik und Weblog von Alban Nikolai Herbst. Dissertation, Universität Göttingen 2015, S. 309.

8 Scheinbar deshalb, weil sich aus der Binnenperspektive, wie gesagt, simulierte und reale Wirklichkeit nicht unterscheiden lassen. 
doch, indem sich, so die These, in Herbsts Fantasie die eigene Identität mit der des von ihm informatisch erzeugten Doppelgängers austauscht. ${ }^{9}$ Ein für Mediennutzer eigentlich typisches Phänomen, das sogar jeder Fernsehzuschauer kennt, wenn sich die eigene Fantasie unentwirrbar mit den im Film gesehenen Charakteren verquickt. All das deutet jedoch im Roman darauf hin, dass die von den Programmierern kodierten kybernetischen Welten, Deters Realwelt (W1) und die Anderswelt (W2), nicht so sind, wie von ihnen gedacht, sondern sich anders verhalten, eben wie sich selbst beobachtende und organisierende Systeme, was beim Ich-Erzähler Herbst zu einem Anflug von Panik führt. Sie wären demnach, wie es im Roman heißt, nicht von ihnen geschaffen und hätten im Grunde schon immer existiert. Es sei nur gelungen, einen Zugangskode zu finden und damit die Möglichkeit, sie zu betreten und mit ihnen zu verschmelzen. ${ }^{10}$

Diese Beobachtung erweist sich insofern als aufschlussreich, als, wie eingangs erläutert, die unterschiedlichen Logiken der Komponenten komplexer Systeme realiter verhindern, dass es zu einem übergangslosen Austausch von Information an den Schnittstellen kommen kann. Wie Armin Nassehi mit Bezug auf das System Gesellschaft gezeigt hat, kommt es an den Stellen zu Interdependenzunterbrechungen, also zu Perspektivendifferenz und damit Bezugsproblemen, wo Funktionssysteme mit verschiedenen Eigenlogiken aufeinandertreffen. ${ }^{11}$

Anders im Roman, wo, solange das Subjekt außer Betracht bleibt, die künstlichen Welten einfach immer wieder umprogrammiert werden können und wo selbst die generierende Matrix des manifesten Textes als Produkt einer programmierten Oberfläche erscheint, wie deutlich wird, wenn ein merkwürdiger Kode den Fließtext durchbricht. ${ }^{12}$ Es sind dies die Stellen, wo aufgrund von Programmfehlern die im Text evozierte, erzählte Wirklichkeit ein- bzw. abbricht. Dies wiederum ist simultan gekoppelt an den Bewusstseinsprozess des Ich-Erzählers Alban Herbst, der just während dieser Unterbrechungen,

Folgt man der Darstellung von Bobzin, so erweisen sich Deters Realwelt (W1) und die Anderswelt (W2) aus dem Vorgängerroman Thetis nunmehr als Computersimulationen in der Welt Garrafff (W3), also der von mir so bezeichneten intradiegetisch realen Welt, in der sich die Beelitzer Firma Cybergen und das Personal der Programmierer einschließlich Herbst befinden. Vgl. Bobzin: Von Bremen in die Anderswelt, S. 294 U. 301.

10 Vgl. A.N. Herbst: Buenos Aires, S. 151.

11 Vgl. A. Nassehi: Letzte Stunde der Wahrheit, S. 89 f.

12 Vgl. A.N. Herbst: Buenos Aires, S. 16 f., 27, 32 f. und passim. 
an denen zugleich die Schnittstelle zwischen informatisch erzeugter Systemrealität und intradiegetisch realer Wirklichkeit sinnfällig wird, von leichten Verwirrungszuständen heimgesucht wird. ${ }^{13}$ Oft ist dieser Shift, wie erläutert, verbunden mit einem Wechsel der Erzählperspektive, die interne Fokalisierung des Ich-Erzählers wechselt in die externe eines personalen Erzählers. ${ }^{14}$ Auf diese Weise wird eine Differenz erzeugt, die ansonsten bei reiner Fokussierung auf den Bewusstseinsprozess eines erzählenden Ichs verschwinden würde.

$\mathrm{Da}$ in der Realität verschiedene Funktionssysteme, wie bereits gesagt, aufgrund ihrer logisch-semantischen Geschlossenheit nicht ohne weiteres sinnvoll miteinander kommunizieren können, erscheint es keinesfalls zufällig, dass in der Theorie wie auch im Roman dem Computer die Rolle der integrierenden Instanz zukommt. So ist es bei Nassehi, mit Dirk Baecker, der Computer, der »durch seine Fähigkeit der Rekombinierbarkeit von Unterschiedlichem $\aleph^{15}$ hinter der sichtbaren Realität eine neue Wirklichkeit aus statistischen Zusammenhängen, Wechselwirkungsprozessen und Strukturähnlichkeiten erzeugt, die Beobachter letztlich überfordere. ${ }^{16}$ Wenn es im Roman als Form der Narration, mit Ricœur gesprochen, zu einer »Synthesis des Heterogenen $\ll^{17}$ kommt, so ist dies zu verstehen als erzähltheoretische

14 Vgl. ebd., S. 16 f. Hier verliert Alban Herbst Hans Deters von seinem Monitor in Beelitz, dem Standort der Firma Cybergen, für die Herbst als Programmierer und wissenschaftlicher Mitarbeiter arbeitet. Er wird dabei von Beutlin, einem holomorphischen Mitarbeiter der SZK (Sicherheitszentrale Koblenz) in Wiesbaden wiederum per Kamerasteuerung und Bildschirm beobachtet. Schon daraus ergibt sich eine komplexe Struktur der Beobachtung eines Beobachters, wie sie etwa in der Systemtheorie von Luhmann eingeführt wurde, auf den sich Nassehi auch in seinen Überlegungen zur gesellschaftlichen Komplexität bezieht.

15 A. Nassehi: Letzte Stunde der Wahrheit, S. 142.

16 Vgl. ebd. Dies ließe sich auch mit McLuhans Vorstellung der »Medien als Übersetzer« formulieren, also etwa der Übertragung von Lautsymbolen in elektronische Information. Dabei kommt es jeweils zu einer Erweiterung des natürlichen, menschlichen Körpers durch technische Innovation. So kann man mit McLuhan den Computer bzw. die damit verbundene Technologie als Erweiterung des zentralen Nervensystems verstehen. Vgl. dazu Marshall McLuhan: Die magischen Kanäle. Understanding Media, 2. erweit. Aufl., hrsg. von Gerti Fietzek und Michael Glasmeier, Dresden et al.: Verlag der Kunst 1995. S. 96-103.

17 Paul Ricœur: Zeit und Erzählung, Bd. 1: Zeit und historische Erzählung, übers. aus d. Franz. von Rainer Rochlitz, München: W. Fink 1988, S. 7. 
Beschreibung der eingangs benannten Fähigkeit der Erzählung, über ontologische Grenzen hinweg sinnhaft Zusammenhänge zu entwerfen. Dies erfolgt über die Erzeugung geschichtenförmiger narrativer Identitäten in dem weiten Sinne, dass, wie Norbert Meuter schreibt, »heterogene Handlungen und Ereignisse zur >zeitlichen Einheit einer vollständigen und umfassenden Handlung`verknüpft werden. $\ll^{18}$ Beide, der Computer wie die Erzählung, leisten also Vergleichbares, nur in völlig verschiedenen Geltungsbereichen - der Computer in der wissenschaftlich-technischen Welt (objektiv), die Erzählung in der zwischenmenschlichen Kommunikation (subjektiv)-, indem sie heterogene Bestandteile $\mathrm{zu}$ einem Ganzen integrieren.

Im Roman Buenos Aires. Anderswelt nun werden beide Arten der Erzeugung von Information bzw. Sinn/Bedeutung miteinander gekoppelt. Wie erläutert, kommt es im Medium der erzählenden Sprache zu einer Verschmelzung informatisch eigentlich getrennter Identitäten, indem nämlich Deters plötzlich auf der Ebene von Herbst erscheint. Im Text heißt es dazu: »- Er war der erste Avatar, der seinen definierten Bezugsrahmen verließ. [...] Welt und Anderswelt verschmolzen. Und dann - erst mit Deters, nun mit Broglier - hatten die Cyberwelten auf Garrafff zugegriffen, als wären wir selbst in einen Zeitbruch gerutscht. ${ }^{19}$

Besonders augenfällig wird die Durchdringung der Welten - diesmal wieder in umgekehrter Richtung - gegen Ende des Romans, wo Herbst die Identität von Deters aus der Anderswelt Buenos Aires löscht, um sich selbst an dessen Stelle in das Café Silberstein zu kopieren, wohin Deters unterwegs war. Es findet ein raumzeitlicher Bruch in der Erzählung statt, der erzähltechnisch dadurch realisiert wird, dass das Ende des 7. Abschnitts im Kapitel ... die Unendlichkeit ${ }^{20}$ erst im letzten Kapitel Vom Silberstein zur Dunckerstraße $e^{21}$ seine Fortsetzung findet:

»Ich lief und stolperte, riß Deter's Klamotten vom Stuhl an mich, mußte mich noch einmal bücken, die Eurocard war runtergerutscht ... dann weg ... dann wieder eine Tür aufgerissen und $(B A, 229)[\ldots]$ Trennen. Ein weiterer Schritt.

18 Norbert Meuter: Narrative Identität. Das Problem der personalen Identität im Anschluß an Ernst Tugendhat, Niklas Luhmann und Paul Ricœur, Stuttgart: M \& P Verlag 1995, S. 129.

19 A.N. Herbst: Buenos Aires, S. 98.

20 Ebd., S. 229.

21 Ebd., S. 257. 
Rechts eine moderne SushiBar, links zweidrei Tische mit hohen Stühlen barhockerar Vorraum, so müde. Elend müde. Das Zeug an mich gerafft. Unterm rechten Arm. Warum schwank ich so? Noch die Eurocard in den Hans Erich Deters das also ich. Seine Klamotten unterm Arm, meine noch an: Die Welt. Ich muß mich umziehen. $\ll^{22}$

Die Auslassungen bzw. Apokopen sind wieder Zeichen der Verwirrung des Protagonisten, der einen raumzeitlichen Shift durchmacht. ${ }^{23}$ Was hier zudem deutlich wird, ist, dass die informatische Schnittstelle ins Erleben des Subjekts verlegt wird. Dies wird noch einmal wichtig werden bei der Frage nach möglichen Deutungsanschlüssen, bei denen komplex rückgekoppelte Beobachtungszusammenhänge mit den daraus resultierenden doppelten bzw. Mehrfachverweisungszusammenhängen eine Rolle spielen.

Der erzähltechnische Sprung entspricht dabei dem, was der Autor Herbst in seinen Poetik-Vorlesungen zum Kybernetischen Realismus als Grundstruktur seines Erzählens bezeichnet, nämlich »Regelkreise[n]«, die als Zyklen »im

22 Ebd.

23 Entscheidend ist nun nicht nur, wie dieser Shift erzählerisch ausgestaltet wird, sondern welche Konsequenzen sich damit über das Erleben des Subjekts hinaus für die Vorstellung einer kohärenten Wirklichkeit ergeben. Der Wechsel des Gerichtet-seins (der Perspektive) von einem Wahrnehmungszentrum (Herbst) in das andere (Deters) hinüber ist bei ANH (empirischer Autor Alban Nikolai Herbst) seit seinem ersten Roman Die Verwirrung des Cemüts ein wesentlicher Gegenstand der erzählerischen Ausgestaltung von Intersubjektivität bzw. deren Simulation, die sich nicht auf der Ebene verbaler Kommunikation vollzieht, sondern im Denken und in der Imagination, also im Raum der Innerlichkeit des Ich-Erzählers resp. der erzählten Figur stattfindet. VgI. Alban Nikolai Herbst: Die Verwirrung des Gemüts, München: List 1983, S. 9, 12, 19, 24 , $58 \mathrm{f}$, 64,66 und passim. Während das Subjekt den Shift von einem Bezugssystem ins andere als momentanen Verlust des eigenen Standorts, gewissermaßen als Dekontextualisierung oder gar Dekonstruktion des eigenen Weltbezugs erlebt, resultiert allgemein gesprochen als Effekt eine Pluralisierung von Wirklichkeit bzw. ihrer Wahrnehmung, die damit in eins fällt. Erweitert man dieses Modell aus der Erzählung, so ergibt sich daraus eine Vorstellung von Wirklichkeit, die sich mit der je besonderen Wahrnehmungsperspektive aller einzelnen Subjekte multipliziert, sodass sich gerade die Idee ihrer Einheitlichkeit von hier aus betrachtet als Fiktion erweist. Wirklichkeit in diesem Sinn ließe sich erst im Prozess des Aushandelns im Rahmen intersubjektiver, kommunikativer Akte herstellen und kann folglich nicht als Cegebenheit vorausgesetzt werden. 
Verhältnis zueinander[...] springen «. ${ }^{24}$ Dies kann vorderhand als Spielart der nicht-linearen Narration verstanden werden, die wiederum typisch für komplexes Erzählen ist. Räumlich gedacht lässt sich diese Struktur auch als Spirale vorstellen, deren Zeitschlaufen jedoch nicht an den Anfang zurückführen, sondern sich »irreversibel über fortlaufende Metaebenen auf dem Zeitstrahl voranbewegen.« Die daraus resultierende Wirkung beschreibt Herbst folgendermaßen: "Hat man den Eindruck, ein Erzählzyklus sei wieder an seinem Anfang angekommen, so haben sich doch sämtliche Beteiligten und hat sich auch das grundlegende Setting verändert. $\ll^{25}$ So reflektiert das Ende des Romans zwar den Anfang, der auch vorm Café Silberstein spielt, wiederum mit Herbst und Deters, gemäß der poetologischen Maxime »[k]eine Bewegung in dem einen Bereich ohne Spiegelung in anderen (Bereichen) ${ }^{26}{ }^{26}$ die das Prinzip kybernetischer Rückkopplung für das literarische Schreiben übernimmt, jedoch sind alle anderen Parameter nicht mehr identisch.

Interessanterweise bleibt dieses Prinzip nun nicht auf den literarischen Text beschränkt, sondern greift über auf das, was ich zu Beginn des laufenden Kapitels seine Randbedingungen genannt habe. Es geht darum, wie Herbst schreibt, dass »die Entstehungsprozesse selbst Teil des Kunstwerks sein müssen«. Dazu zählt natürlich das Autoren-Ich, welches, wie er weiter ausführt, »in einer adäquaten nachpostmodernen Dichtung notwendigerweise selber zum Gegenstand des Romans wird, zu einer Figur, die sich von den anderen Figuren nicht mehr strukturell unterscheidet $«{ }^{27}$ Dies ist letztlich wohl der Grund dafür, warum eine Figur namens Alban Herbst auf der intradiegetischen Ebene des Romans erscheint, der Autor damit also eine absteigende, metaleptische Grenzüberschreitung von der Extra- auf die Intradiegese unternimmt und in seinen eigenen Text migriert. ${ }^{28}$ Doch geht die komplexitätserweiternde Hereinnahme von extradiegetischer Wirklichkeit noch weiter, wenn der Autor davon spricht, dass alles, was ihm während des Schreibens

\footnotetext{
24 Alban Nikolai Herbst: Kybernetischer Realismus. Heidelberger Vorlesungen, Heidelberg: Manutius 2008., S. 81.

25 Ebd., 82.

26 Ebd., 81.

27 Ebd., 91.

28 Zum Phänomen der erzählerischen Metalepsen und den damit verbundenen semantischen Gehalten vgl. Sonja Klimek: Paradoxes Erzählen. Die Metalepse in der phantastischen Literatur, Paderborn: Mentis 2010, S. 70. Zur absteigenden Metalepse, wie sie hier vorliegt, vgl. insbesondere ebd.
} 
geschieht, in den Text hineingenommen werde. ${ }^{29}$ Tendenziell gilt das auch für die Wirklichkeit als Allgemeinbegriff, der im Kybernetischen Realismus, laut Herbst, den lebenswirkenden Prozess sämtlicher wechselwirkenden, äußeren und inneren Einflüsse, die während des Schreibprozesses mitlaufen, meine. ${ }^{30}$

Dass es im Roman Buenos Aires. Anderswelt keine Zentralperspektive gibt, sondern stattdessen verschränkte Beobachtungsverhältnisse, d.h. ein Reziprokwerden von Beobachten und Beobachtet-Werden, entspricht dem eingangs konstatierten Fehlen einer zentralen Instanz in komplexen Systemen. ${ }^{31}$ Etablieren sich im Roman aufgrund dieser Konstellation nach Bobzin »Beobachternetzwerke «, ${ }^{32}$ die dazu führen, dass die Programmierer in Garrafff (W3) von ihren eigenen Simulationen beobachtet werden können, ${ }^{33}$ so ergeben sich daraus verschiedene, im Roman realisierte Kombinationen. ${ }^{34}$ Doch damit nicht genug, auch Möglichkeiten, die, wie Bobzin schreibt, ausgelassen werden aber gleichwohl bestehen, »indem beispielsweise Deters Goltz dabei beobachtet, wie dieser Herbst dabei beobachtet, wiederum Deters zu beobachten. $\ll^{35}$

In diesem Fall handelt es sich um einen komplex zurückgekoppelten Beobachtungszusammenhang, bei dem nicht mehr entscheidbar ist, welche Ebene der Beobachtung hierarchisch über den genannten anderen Positionen angesiedelt wäre. ${ }^{36} \mathrm{Da}$ das, was hier beobachtet wird, nicht mehr eindeutig zuschreibbar ist, wäre dies nun ein Beispiel dafür, »dass etwas gleichzeitig unterschiedliche Zustände annehmen kann ${ }^{37}$ - hier der Romantext inklusive der in ihm angelegten möglichen (Bedeutungs-)Zustände -, was nach

Vgl. A.N. Herbst: Kybernetischer Realismus, S. 92.

30 Vgl. ebd., S. 99.

31 Beides, sowohl der Verzicht auf eine Zentralperspektive als auch die Verschränkung der Beobachtungsverhältnisse, wird von Kreknin sowie von Bobzin mit Malsch eingeräumt. Vgl. I. Kreknin: Poetiken des Selbst, S. 368 f. Vgl. außerdem Bobzin, Von Bremen in die Anderswelt, S. 308.

Ebd., S. 306.

33 Vgl. ebd., S. 305.

34 Vgl. ebd.

35 Ebd., S. 306.

36 Das Modell eines solchen rückgekoppelten Beobachtungszusammenhangs findet sich bereits in Die Verwirrung des Cemüts. Dort heißt es: »Das Modell: Wie ich mir denke, wie Falbin sich Laupeyßer denkt. Wie ich mir denke, wie Falbin sich Laupeyßer denkt, wie dieser mich denkt. Wie Laupeyßer sich denkt, wie ich mich denke. Soviel ist klar.«A.N. Herbst: Verwirrung des Cemüts, S. $73 \mathrm{f}$.

37 A. Nassehi: Letzte Stunde der Wahrheit, S. 120. 
Nassehi ein Hauptkriterium für Komplexität darstellt. ${ }^{38}$ Das gilt sowohl für die komplex rückgekoppelte Beobachtung als auch für die Durchdringung der Identitäten von Herbst und Deters.

Diese Möglichkeit, verschiedene Sinnzusammenhänge an den gleichen Text anzuschließen, lässt sich zudem mithilfe des Aspektsehens veranschaulichen, wie es von Wittgenstein philosophisch im Kontext der Interpretation sprachlicher oder auch musikalischer Bedeutungen herangezogen wird. ${ }^{39}$

38 Vgl. ebd. Nassehi beschreibt dies mithilfe der Metapher der verteilten Intelligenz. Diese erfordert Kommunikation zwischen Komponenten mit unterschiedlichen Selektivitäten, die eben Signale nur nach eigenen Regeln interpretieren. Information entsteht also erst dann, wenn Signale an Schnittstellen im Sinne der angeschlossenen Komponente, d.h. nach ihren Regeln, sinnvoll verarbeitet werden können. Umgekehrt bedeutet es, dass das gleiche Signal, wenn es an unterschiedlichen Stellen, also durch verschiedene Komponenten interpretiert (dekodiert) wird, auch Unterschiedliches bedeutet. Vgl. ebd., S. 119 f. Man spricht in diesem Zusammenhang auch von Mehrfachkodierung bzw. »Polykontexturalität«. Ebd., S. 67.

39 Vgl. Joachim Schulte: Wittgenstein. Eine Einführung, Stuttgart: Reclam, 2009, S. 203210. Das Aspektsehen ist, obwohl darauf selten rekurriert wurde, bereits in der surrealistischen Malerei von Salvatore Dali als Methode der Darstellung umgesetzt worden. In Cemälden wie Spanien (1938), Der große Paranoiker (1936), Endloses Rätsel (1938) oder Die drei Lebensalter (1940) kann der Betrachter zwischen zwei Möglichkeiten der Wahrnehmung wählen bzw. changieren. Diese von Dali selbst so sogenannte paranoisch-kritische Methode wechselt also bereits auf der Ebene der ikonischen Darstellung vom Modus der Eindeutigkeit in den der Mehrdeutigkeit. Man kann etwas sso oder so s sehen, wie ein in einem Paradox oder Widerspruch gefangener naiver Beobachter, der die Trivialität des Eindeutigen im Denken nicht in Richtung einer weiteren, damit komplexeren Identität zu transzendieren vermag. Hier zeigt sich, dass gegensätzliche Wahrnehmungen gleichzeitig wahr sein können, wenn der Wahrnehmungsinhalt mehrfachkodiert ist und daher gleichzeitig unterschiedliche Bedeutungszustände annehmen kann (s.o.). Grundsätzlich sind natürlich die mehrfachkodierten Kippfiguren der natürlichen, visuellen Wahrnehmung zu unterscheiden von den Signalstrukturen, die an den Schnittstellen zwischen künstlichen (digitalen) Systemen und Benutzersubjekten desambiguiert werden müssen. Das Phänomen als solches ist auch aus dem linguistischen Sprachverstehen, einem Teilprozess der Sprachverarbeitung, bekannt, wo sowohl auf phonetischer als auch auf der Ebene des schriftlichen Zeichens material gleiche Bedeutungselemente (Laute bzw. Lettern) je nach Verwendung im System einer bestimmten Sprache anders desambiguiert werden. Selbst auf Wortebene gibt es in seltenen Fällen identische Lautverkettungen, die jedoch von Sprachsystem zu Sprachsystem andere Bedeutungen haben. Allein aufgrund der Möglichkeit, dass sprachliche Elementarteilchen gleichzeitig unterschiedliche Bedeutungszustände annehmen können, entsteht eine Herausforderung durch Komplexität. 
Daraus ergibt sich mindestens ein doppelter Verweisungszusammenhang, der einmal der von Nassehi geprägten Formel für Mehrfachkodierung entspricht, dass etwas gleichzeitig unterschiedliche Bedeutungszustände annehmen kann und sich zum andern, gestalttheoretisch und damit rezeptionsästhetisch gewendet, vergleichbar den Wittgensteinschen Kippfiguren, unter verschiedenen Aspekten sehen lässt.

So spricht neben seiner ironischen Haltung vor allem der Umstand, dass der Wechsel zwischen den Schnittstellen ins Erleben des erzählenden Subjekts verlegt ist, dafür, dass sich die Identitäten lediglich in der Fantasie des Erzählers austauschen. Auf diese Weise kann der zugrundeliegende, psychische Wirkzusammenhang für das vorderhand erzählerisch dargestellte, futuristische Szenario in den Fokus gerückt werden. Denkbar wäre jedoch auch, dass die dehierarchisierende Wirkung komplexer Beobachtungszusammenhänge, wie sie im Roman entfaltet werden, aus der technisch weit fortgeschrittenen Architektur von Computernetzwerken resultiert. Beide Möglichkeiten lassen sich, wie bereits angedeutet, natürlich auch verschränken, wobei allerdings die Grenzen zwischen verschiedenen Geltungsbereichen, wiederum metaleptisch, überschritten werden. So stellt der Romantext zweifellos die erzählerische Fantasie des realen, textexternen Autors Alban Nikolai Herbst dar. Intradiegetisch sind die technischen Bedingungen jedoch so weit fortgeschritten, dass die beschriebenen Phänomene des Ebenenwechsels unabhängig davon auftreten. Verschränkt werden beide Perspektiven durch die Figur Alban Herbst, der als Alter ego des Autors jenes Szenario psychisch erlebt. Dies ergibt rezeptionsästhetisch betrachtet eine Konstellation, die als Beobachtung dritter Ordnung ${ }^{40}$ verstanden werden kann: Der Leser beobachtet den Autor, der seine Figur beobachtet, die wiederum, indem sie beobachtet, eine Unterscheidung zwischen realer und simulierter Wirklichkeit zu machen versucht.

Wenn Komplexität im Roman wie in der modernen Gesellschaft durch exakt solche Strukturen geprägt ist, stellt sich mit Nassehi die Frage, wie »die Konflikte zwischen den unterschiedlichen Intelligenzen und Funktionen als Übersetzungskonflikte zu rekonstruieren sind . ${ }^{41}$ Dies trifft genauso auf die Akteure im Roman zu, die wie Markus Goltz und Alban Herbst mit dem Pro-

40 Der Begriff versteht sich als Iteration der Beobachtung zweiter Ordnung aus Niklas Luhmanns Theorie der Beobachtung.

41 A. Nassehi: Letzte Stunde der Wahrheit, S. 199. 
blem konfrontiert sind, »wer welchem Bezugssystem entstammt $«{ }^{42} \mathrm{Kommu-}$ nikation zwischen den Ebenen, wie im Fall von Herbst und Deters, ist nur als Übersetzung denkbar, und zwar nicht im Sinne einer simplen Koppelung unterschiedlicher ontologischer Domänen, sondern vielmehr als Management von disruptiven Unterbrechungen, wie sie Herbst bei seiner Flucht aus Garrafff (W3) zurück in Deters Realwelt Buenos Aires (W1) erlebt.

Geht man davon aus, wie sich Komplexität und die damit verbundenen disruptiven Brüche, siehe Herbst, anfühlt, wie sie erlebt wird, so ist Komplexität als gesellschaftliche und individuelle Herausforderung zumeist mit einer Überforderung verbunden, die das Subjekt, etwa in der Arbeitswelt des Neoliberalismus, mit widersprüchlichen Anweisungen konfrontieren - zum Beispiel durch gleichzeitige Beschäftigung in verschiedenen Branchen - und vom Ich als zentraler Instanz der Lebensführung nicht mehr integriert werden können. Es fragt sich, wie der damit verbundene Verlust an Autonomie, verstanden als Fähigkeit zur Selbstermächtigung, zurückgewonnen werden kann. Im Roman spiegelt sich dies, indem das Subjekt zum Schauplatz psychischer (Herbst) und physischer Transmutationen (Borkenbrod) wird. Systemgrenzen und Beobachtungshorizonte verschränken sich in einer Weise, die zwar überraschende Vieldeutigkeit mit sich bringt, ohne dass jedoch daraus vorderhand weitere Handlungsmöglichkeiten entspringen. Es resultiert vielmehr »ein Darstellungsproblem « ${ }^{43}$ für die Komplexität der Verhältnisse, von dem auch Nassehi spricht, weil die Analyse des Problems nicht in eine appellative Form zu bringen ist, die mit konkreten Forderungen verbunden werden könnte. ${ }^{44}$ Es werden Möglichkeiten durchgespielt, die aber an der herrschenden Entwirklichung und dem Verlust homogener, autonomer Subjektivität nichts ändern. So fragt sich etwa, warum Herbst Deters am Ende des Romans löscht und sich selbst an seine Stelle kopiert. Verbindet sich damit nicht die Hoffnung, dass kybernetische Operationen auf die Existenz des Subjekts selbst angewendet, nicht vielleicht zu ungeahnten Freiheiten jenseits des Materiellen führen könnten?

Im Roman jedoch nimmt diese Möglichkeit kaum Gestalt an. Stattdessen werden am Schluss, als Herbst zurück in der Dunckerstraße 86, der Adresse von »Herbst \& Deters Fiktionäre«, den leeren Briefkasten öffnet und wieder verschließt, alle in den verschiedenen Simulationen gleichzeitig stattfin-

\footnotetext{
42 A.N. Herbst: Buenos Aires, S. 101.

43 A. Nassehi: Letzte Stunde der Wahrheit, S. 201.

44 Vgl. ebd.
} 
denden Ereignisse in parodistischer Absicht zitiert und zudem die Idee eines Identitätswechsels in den simulierten Welten karikiert, ${ }^{45}$ die von Herbst, wie im gewöhnlichen Leben, auch hier nur durch eine Überdosis Alkohol realisiert werden kann. Die uneigentliche Redeweise der Parodie verbirgt hier, dass es im Zustandsraum der Simulation keinen Standort mehr gibt, der es gestatten würde, von außen zu beobachten, wie die Avatare/Gegenstände mit ihren Repräsentationen übereinstimmen. Das Subjekt ist zwar in einem sich immer weiter ausdifferenzierenden, fraktalen Raum entgrenzt, die von ihm erlebte Wirklichkeit aber deswegen entregelt, mit anderen Worten chaotisch komplex. Unter den Bedingungen weit fortgeschrittener Digitalisierung, wie sie das Szenario des Romans entfaltet, ist die Möglichkeit des Subjekts, mit sich selbst zu kommunizieren, überhaupt noch Kontakt zu sich zu bekommen, nicht mehr anders zu haben als mithilfe komplexer Übersetzungsstrategien. Somit ließe sich auch das Handeln von Herbst allenfalls als Versuch verstehen, zu sich selbst zu kommen, weil die Möglichkeit, sich unmittelbar erreichen zu können, eben nicht mehr gegeben ist. In diesem Sinn wäre es auch, wie zuvor erläutert, eine Reaktion gegen den drohenden Verlust von Autonomie durch eine übermächtig bzw. unberechenbar gewordene Technologie, mithin ein Akt der Selbstermächtigung. 



\section{Aspekte der Rezeption komplexer Erzähltexte}

Im Anschluss an die im engeren Sinn literarische Analyse liegt es nahe, sich an dieser Stelle eingehender mit der ästhetischen Wirkung komplexen Erzählens auf den Leser zu befassen. Interessanterweise fehlen nicht nur für diese Fragestellung, sondern überhaupt für den Begriff der Komplexität, sowohl im engeren Bereich der Literaturwissenschaft als auch für den Bereich ästhetischer Phänomene allgemein, bisher weitgehend Beiträge aus der Forschung. ${ }^{1}$ Literaturwissenschaftlich betrachtet ist die Forschungslage sogar mehr als dünn. In Ermangelung einschlägiger Publikationen ist deswegen von Mieths These der vielfältigen Erweiterung von Erfahrung, Verstehen und Handlungsmotivationen beim Leser auszugehen. Bezeichnend ist hier auch, dass Mieths Ansatz kein genuin literaturwissenschaftlicher ist, sondern aus der Ethik, genauer gesagt der narrativen Ethik, kommt. Erzählen allgemein und komplexes Erzählen im Besonderen wird also verortet im Zusammenhang des Handelns und dabei mit bestimmten Funktionen assoziiert, die vom Unterhaltungsinteresse bis zur Weitergabe von Erfahrung und der Motivation für bestimmte Handlungsweisen reichen. ${ }^{2}$

Mieth äußert sich nun über die Wirkung resp. Funktion des komplexen Erzählens in folgender Weise:

»Ein offensichtliches Merkmal der Komplexität des Erzählens ist die wechselseitig kritische Relation und partielle Aufhebung der Vielschichtigkeit der

$1 \quad$ Von dieser Lage geht auch Albrecht Koschorke aus, der in seiner Einleitung zum Tagungsband Komplexität und Einfachheit Definitionsvorschläge für ästhetische Komplexität macht, auf die ich mich im Folgenden noch beziehe. Vgl. Albrecht Koschorke (Hg.): Komplexität und Einfachheit. DFG-Symposium 2015, Stuttgart: J.B. Metzler 2017, S. 1-10, hier $6 \mathrm{f}$.

2 Vgl. D. Mieth: Moral und Erfahrung, S. 97-102. 
Perspektiven, die in den Erzählgang eingeflochten werden. Dabei geht es jedoch nicht einfach um Harmonie und Ausgewogenheit, sondern um eine bestimmte Weise der Provokation, die es vermeiden soll, mit dem Erzählvorgang Proselyten von Überzeugungen und Nachahmer von Handlungen zu schaffen. Dass komplexes Erzählen oft als destruierend erscheint, hat mit diesem seinem provokativen Charakter zu tun. Der implizite Leser soll kein geschlossenes Weltbild erhalten, er soll ständig erneut angetrieben werden, aus fixiertem Verstehen aufzubrechen und neue Möglichkeiten der Erfahrung, des Verstehens und der Motivation zu suchen. $\ll^{3}$

Was in dieser Beschreibung auffällt ist zweierlei. Zunächst, dass es bei der Lektüre komplexer Erzählungen selbst wieder zur Reduktion kommt, weil die u.U. vielfältigen Perspektivendifferenzen als mitlaufende $\mathrm{zu}$ verwirrend wären und den notwendigen Kohärenzaufbau empfindlich stören müssten. Schließlich, dass der Leser, weil er im Text immer wieder mit dieser Differenzerfahrung konfrontiert wird, sich einer beständigen Provokation ausgesetzt sieht, die eine vorschnelle Verfestigung des Verstehens, der Erfahrung und sogar der eigenen Handlungsmotivationen über den Text verhindern soll. Es wird also einer Dynamisierung das Wort geredet, die sich vielleicht, denkt man an die Anderswelt-Romane von Herbst, als Leseerlebnis von ineinander stürzenden, sich verschlingenden Identitäten (wahrgenommen als Intensität miteinander konkurrierender Kohärenzen) umschreiben ließe und somit den disruptiven Erfahrungen von Mediennutzern wie auch der Hauptfigur in Buenos Aires. Anderswelt entspräche.

Eine solche verunsichernde oder gar beunruhigende Leseerfahrung, die je nach lebensweltlichem Kontext sogar, wie Mieth meint, zur geistigen Mobilisierung des Lesers führen kann, wäre sicher für ein Lesepublikum, das Lektüre eher im Sinn einer zerstreuenden Freizeitbeschäftigung versteht, kaum angemessen. Im Zusammenhang der kreativen Aneignung von literarischen Texten ist sie jedoch äußerst wünschenswert. Ähnlich wie beim kreativen Umgang mit Situationen im Alltagsleben oder in der Auseinandersetzung mit der Umwelt kommt es hier, wie wir u.a. aus der Neuroethik wissen, darauf an, »eine bestehende Situation anders zu deuten. « ${ }^{4}$ So erläutert der Neurophilosoph Detlev Linke weiter, dass im kreativen Prozess »nicht einfach aus dem

3 Ebd., S. 104.

4 Detlev B. Linke: Die Freiheit und das Gehirn. Eine neurophilosophische Ethik, München: C.H. Beck 2005. S. 23. 
Nichts etwas Neues erzeugt wird, sondern vielmehr das Gegebene neu interpretiert wird. $\aleph^{5}$ Damit kann die Fähigkeit zur »Uminterpretation«, ${ }^{6}$ d.h. »etwas Gegebenes in einen neuen Interpretationszusammenhang [zu übersetzen] «, ${ }^{7}$ als wesentlich für Kreativität allgemein bzw. für kreatives Lesen im Besonderen betrachtet werden. Dass es sich bei dieser Aktivität nicht nur um eine rein kontemplative Haltung handelt, sondern um die Voraussetzung für ein verändertes Handeln, wird einsichtig, wenn man sich klar macht, dass mit der Uminterpretation von Gegebenheiten, hier dem erzählten Geschehen eines literarischen Textes, zumeist ein Perspektivenwechsel resp. eine Perspektivenerweiterung verbunden ist. Auf welche Weise der zuvor untersuchte literarische Text vom Leser beides verlangt, dürfte am Beispiel des Wechsels der Erzählperspektive von der 3. in die 1. Person, der Mithereinnahme von Randbedingungen seiner Entstehung in den Text und schließlich der komplex rückgekoppelten Beobachtungszusammenhänge deutlich geworden sein.

Bringt man dieses Ergebnis versuchsweise einmal mit dem zusammen, was Alexander Koschorke als Maß ästhetischer Komplexität definiert, so ergeben sich daraus überraschende Korrespondenzen. Angelehnt an kybernetisch-sozialwissenschaftliche Definitionen des Komplexen formuliert er entsprechend systemisch: »Das Maß für ästhetische Komplexität wäre dann die Verwebungsdichte möglicher oder aktualisierter Relationen zwischen den Elementen, die das Kunstwerk umfasst. $\ll^{8}$ Hier liegt es nahe an den komplex rückgekoppelten Beobachtungszusammenhang zwischen Deters, Goltz und Herbst zu denken, bei dem, wie gezeigt, unterschiedliche Ebenen überschritten bzw. miteinander verschränkt werden. Aber auch die Interpretation des Referenzwechsels beim Austausch der Personalpronomen sowie die metaleptische Hereinnahme der Identität des realen Autors in die Diegese können in diesem Sinn als Elemente des literarischen Textes verstanden werden, die mit Nassehi simultan unterschiedliche Bedeutungszustände annehmen können, weil sie sich unterschiedlich relationieren lassen. So ist Alban Herbst, wenn auch leicht verkürzt, gleichzeitig der Name des realen Autors und der Figur des Programmierers in Garrafff, und so kann das erzählte Geschehen erlebnismäßig, wie erläutert, sowohl Deters als auch Herbst und über die Namensgleichheit sogar dem realen Autor zugeschrieben werden, während

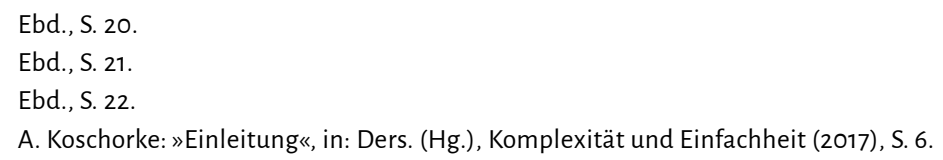


bei der Beobachtungskaskade der Inhalt der Beobachtung allen beteiligten Beobachtern zukommt. Das alles macht das Erzählen komplex, denn je nachdem, welche Perspektive gerade eingenommen wird, wechselt auch die Sicht auf die erzählte Welt, erscheinen Beziehungen $\mathrm{zu}$ anderen Figuren bzw. zu den gleichen Figuren anders oder gar nicht.

Auch hier bietet sich wieder ein Konnex zur Hirnforschung an. Wenn die zuvor beschriebene Dynamik aus der Kollision verschiedener Sichtweisen resultiert, so findet sich ein vergleichbares Phänomen bei der Projektion von Abbildungsprozessen im menschlichen Gehirn. Auch dort kommt es zur »Kollision« bzw. zum »Clash «, ${ }^{9}$ wie Linke schreibt, wenn sich formierende Abbildungsprozesse auf bereits strukturierte Neuronensysteme projizieren wollen. ${ }^{10}$ Kreative Lösungen, d.h. in unserem Zusammenhang neue Sichtweisen bzw. Perspektivierungen, entstehen dann, wenn durch die Steigerung der inneren Aufmerksamkeit auf solche Kollisionen neue, bisher nicht wahrgenommene Zusammenhänge sichtbar werden. ${ }^{11}$ Auf der Ebene des Bewusstseins entspricht diesem Geschehen in der Hirnphysiologie ein vergleichbarer Zusammenprall. Es kommt, wie Linke ausführt, zu einem »Clash der Vorstellungen ${ }^{12}{ }^{12}$ Dabei entstehen Vorstellungen nicht einfach aufgrund der Kohärenz von Nervenreizen, ausgelöst beispielsweise durch die Regelmäßigkeit sprachlicher Rhythmen, sondern gerade durch die Kollision verschiedener, miteinander konkurrierender Kohärenzen. ${ }^{13}$ Dies bedeutet im Grunde, dass die Entstehung von Vorstellungen konsubstanziell mit einem solchen Clash verbunden ist, der für kurze Zeit Chaos im Gehirn auslöst. Demnach ist das Gehirn sogar, um überhaupt neue Vorstellungen bilden zu können, auf die Erzeugung von Chaos angewiesen. ${ }^{14}$ Solche ungeordneten Zustände, die zwi-

\footnotetext{
9 D.B. Linke: Freiheit und Gehirn, S. 49.

10 Vgl. ebd.

11 Vgl. ebd.

12 Ebd., S. 67.

13 Vgl. ebd., S. 49.

14 Einen vergleichbaren Gedankengang entwickelt Johannes Wagemann, der auch davon spricht, dass die Bildung von Bewusstsein, d.h. einer kohärenten, kognitiven Ganzheit des Selbst, erst durch Wahrnehmungskrisen herausgefordert wird, welche dessen Integrität immer wieder in Frage stellen. Deshalb spricht er davon, dass das Cehirn gerade nicht Ordnung, sondern im Cegenteil Chaos erzeugt, »nicht Zusammengeführtes, sondern Einzelnes, Dekomponiertes.«»]ohannes Wagemann über Nahtoderfahrungen, das Bewusstseinsorgan und überforderte Schimpansen«, in: Matthias Eckholt, Kann sich das Bewusstsein bewusst sein? Gespräche mit Dirk Baecker et al., Heidelberg: Carl-Auer 2017, S. 158-178, hier S. 169. Er versteht Bewusstsein, das er als umfas-
} 
schen sich formierenden Vorstellungsgestalten auftreten, werden benötigt, damit der Vorstellungsablauf nicht stereotyp, sondern flexibel verläuft, d.h. auch Raum für Korrekturprozesse bleibt. ${ }^{15}$ Analog zur Chaostheorie könnte man dann auch davon sprechen, wie Linke mit Skarda und Freeman ausführt, dass durch Zunahme einer chaotischen Dynamik an den Instabilitätspunkten der Übergang zu einer neuen Ordnung generiert wird. ${ }^{16}$

Übertragen auf die Lektüre des Romans Buenos Aires. Anderswelt lässt sich nun feststellen, dass es auch dort zu den benannten Kollisionen in Form von Konflikten bei der Kohärenzbildung kommt. Ob der Leser imstande sein wird, die zuvor genannten Deutungsalternativen an den Instabilitätspunkten des Textes, also den Stellen, wo es zu Interdependenzunterbrechungen kommt, zu selektieren, wird auch davon abhängig sein, inwiefern er bereit bzw. überhaupt in der Lage ist, seine inneren Zustände und Empfindungen, die den Prozess der Lektüre begleiten, zu bewerten. Wenn er den Clash unterschiedlicher Kohärenzen in der erzählten Wirklichkeit gar nicht realisiert, wird es auch in seinem Bewusstsein nicht zu konkurrierenden Vorstellungsbildungen kommen. Nimmt er die Kollisionen jedoch wahr, stellt sich die Frage, wie er sie beurteilt, d.h. hier im Zusammenhang des Textes interpretiert. Diese Fokussierung auf innere Prozesse, die beim Leser durch alternative Kohärenzen angestoßen werden, kann vermutlich als wichtigste rezeptionstheoretische Implikation komplexen Erzählens angesehen werden. Verläuft dieser Prozess erfolgreich, dies wäre dann der Fall, wenn die Lektüre trotz auftretender Widerstände resp. Verständnisschwierigkeiten nicht abgebrochen wird, so entwickelt sich beim Leser eine Fähigkeit zur Selbstbeobachtung, die systemisch als Beobachtung zweiter Ordnung anzusprechen ist. ${ }^{17}$ Der Leser beobachtet, was während der Lektüre in ihm vorgeht, bewertet die vom Text hervorgerufenen Vorstellungen, ergänzt Leerstellen, zieht Schlussfolgerungen etc. Dies wäre dann auch eine Beschreibung für das, was man als performatives Lesen bezeichnen kann. Dazu gehört die Auseinandersetzung mit performativen Widersprüchen, die an den Stellen auftreten, wo, wie gesagt, verschiedene

sendes Erleben von Sinnstrukturen beschreibt, abgekoppelt von der Materialität des Gehirns im Sinne eines globalen Zustands, gar einer kosmischen Qualität, weil es nirgendwo, auch nicht im Cehirn, lokalisierbar sei. Vgl. ebd., S. 175.

16 Vgl. ebd., S. 72 f., 78 u. 82.

17 Der Begriff stammt aus Niklas Luhmanns Theorie der Beobachtung. Vgl. dazu Detlev Krause: Luhmann-Lexikon. Eine Einführung in das Cesamtwerk von Niklas Luhmann, 2. vollständig überarb., erweit. und aktual. Aufl., Stuttgart: Enke Verlag 1999, S. 56-63. 
Vorstellungen miteinander konkurrieren und es deswegen zu verwirrenden Interferenzen kommt. ${ }^{18}$

18 Performatives Lesen gewinnt sicher in den Fällen an Relevanz, wo die Rezeption des literarischen Textes mit der gegenwärtigen Lebenssituation des Lesenden eine produktive Verbindung einzugehen vermag in dem Sinn, dass ausgehend vom Rezipienten ein emergenter Prozess angestoßen wird, der sich im besten Fall in Richtung der Erzeugung neuer Bedeutung und damit entsprechend neuer Kohärenz auswirkt. Dabei können vom Rezipienten bisher nicht wahrgenommene Verhaltensoptionen geprüft werden, welche im Prozess des Auspendelns eigener Lektüreerlebnisse und kognitiv dissonanter Verhältnisse im eigenen Leben hervorgehen, die geglättet werden wollen. 


\section{Vorläufige Bilanz - Gründe für das gegenwärtige Forschungsdefizit}

Wie sich nunmehr abzeichnet, gilt der Satz, dass eine Zunahme von Komplexität in der Wirklichkeit gleichbedeutend mit der Steigerung von Komplexität in den Verfahren der Komplexitätsbewältigung ist, ${ }^{1}$ auch für die literaturwissenschaftliche Analyse. Für das komplexe Erzählen trifft somit zu, dass insgesamt auch die Deutungs- resp. Anschlussmöglichkeiten an verschiedene Kontexte zunehmen, wenngleich es im Rezeptionsvorgang aus Gründen des Kohärenzaufbaus prozessgemäß auch zur Reduktion kommen kann. ${ }^{2}$ Das Fehlen einer zentralen Instanz, die dem Leser die Entscheidung darüber abnähme, was in der erzählten Welt real, was fiktiv bzw. der Fantasie des Erzählers geschuldet ist, führt zur Bildung unterschiedlicher, einander partiell widersprechender Hypothesen. Der kommunikationstheoretische Befund Nassehis, dass etwas je nach Kopplung simultan unterschiedliche Bedeutungszustände annehmen kann, führt dabei zusammen mit der gestalttheoretischen Einsicht, dass sich das Gleiche unter verschiedenen Aspekten sehen lässt, zu unterschiedlichen Sinnverkettungen bzw. Verweisungszusammenhängen oder auch konfligierenden Kohärenzbildungen. Im vorliegenden Fall kommt

Vgl. A. Koschorke: »Einleitung«, in: Ders. (Hg.), Komplexität und Einfachheit (2017), S. 2.

2 Damit es nicht zu einer paranoiden Dynamik der Überbietung von Komplexität durch noch komplexere Methoden ihrer Bewältigung kommt, ist zu bedenken, dass Komplexitätssteigerung und ihre Reduktion als miteinander verschränkt gedacht werden müssen. Sie bilden ein Begriffspaar, dessen Positionen miteinander interagieren. Denn dies geschieht innerhalb eines Denkens, das, wie Niklaus Largier feststellt, »immer neu Komplexität produziert, wo es sich selbst als Vereinfachung darstellt. « Niklaus Largier: „Sektion 1: Praktiken der Einfachheit. Einführung«, in: Koschorke (Hg.), Komplexität und Einfachheit (2017), S. 11-18, hier S. 15. 
es zur Kollision zwischen fantasiebasiertem Modus (Austausch der Identitäten von Herbst und Deters in Herbsts Fantasie) und systeminterner Interdependenzkopplung (es gibt technisch realisierte Schnittstellen zwischen den verschiedenen simulierten Welten). ${ }^{3}$ Die Verschränkung beider Möglichkeiten erlaubt nun den Vergleich zu den für komplexe Systeme typischen Fluktuationen, die beim Übertritt in eine neue Phase an den Bifurkationspunkten (Phasenübergänge) auftreten. ${ }^{4}$

Wie geschildert, ist dies der Fall, als sich Alban Herbst, bedroht durch die Möglichkeit der Avatare, die Grenze der simulierten Welten zu überschreiten, $\mathrm{zu}$ retten versucht, indem er sich aus Garrafff löscht und an die Stelle von Deters in dessen Realwelt kopiert. Die rein technische Beschreibung des Vorgangs im Roman lässt zunächst vom disruptiven Chaos im Inneren des Subjekts nichts ahnen, ${ }^{5}$ das aber dann zum Schluss am eingestreuten Programmkode und den fragmentierten Wahrnehmungen des Protagonisten kenntlich ist. Da jedoch auch W1 nur eine künstliche Welt ist, scheint dieser Versuch, sich als autonomes Subjekt retten zu wollen, von vornherein zum Scheitern verurteilt, die Handlung von Herbst somit eine reine Kurzschlusshandlung, so stellt es sich auch im Roman dar, mithin eine systeminterne Rückkopplung des informatischen Netzwerks, aus dem es kein Entkommen mehr gäbe. Ob sich das Ganze nun in der - zugegeben verzweifelten - Fantasie des Erzählers und - iterativ erweitert - in der des realen Autors Herbst (als Randbedingung mit allen Implikationen) abspielt oder rein künstlich realisiert wäre, die Figur des Erzählers Herbst selbst ein Avatar, die Schaltkreise seiner Neuronen

3 Es handelt sich hier um die sogenannten »Lappenschleusen«. Vgl. dazu das Beiheft von Buenos Aires. Anderswelt betitelt WurmVorsatz, S. IX.

Zur besseren Anschaulichkeit kann dazu das Feigenbaumdiagramm aus dem WIKIPEDIA-Artikel »Feigenbaum-Konstante« herangezogen werden, https://de.wikipedia.org/w iki/Feigenbaum-Konstante vom 16.02.2021. Vgl. auch die Darstellung, die ich im Kontext der Analyse nicht-linearer Szenarios im Film verwendet habe. André Steiner: $\gg$ Filmisch inszenierte Simultaneität als Mittel der Darstellung nichtlinearer Szenarios«, in: Philipp Hubmann/Till Julian Huss (Hg.), Simultaneität. Modelle der Cleichzeitigkeit in den Wissenschaften und Künsten, Bielefeld: transcript 2013, S. 381-399, hier S. 391.

5 Hier wird zudem deutlich, wie die Vorstellungswelten von Informatik und Biologie fusioniert werden: „Deters' Sachen, aus der Archivdatei, verschob Herbst in die letzten paar Kilobytes vorm SILBERSTEIN. Erst freilich hatte er eine Selbstkopie auf F verfertigt, sich dann in das Datenneuron eingeführt, aus welchem Deters gelöscht worden war, wonach ein vorherprogrammierter ZeitschaltungsBefehl ihn seinerseits aus Garrafff löschte.«A.N. Herbst: Buenos Aires, S. 228. 
technisch assemblierte wären - wird an diesem Punkt unentscheidbar. Dieses Protosystem der Interpretation oszilliert gewissermaßen zwischen diesen beiden Möglichkeiten, hat also schließlich einen mehr oder minder stabilen Zustand erreicht, in dem chaostheoretisch betrachtet die beschriebenen Attraktoren das Geschehen in der Rezeption bestimmen. Komplexe Phänomene, hier das komplexe Erzählen, und die Muster ihrer Interpretation resp. Bewältigung spiegeln sich, das wird nun sichtbar, gewissermaßen auch im Maß ihrer Differenziertheit ineinander.

Es stellt sich damit die Frage, ob nicht die zuvor von Koschorke übernommene Definition ästhetischer Komplexität zu kurz greift, wenn sie sich auf die Dichte der Bezugnahmen zwischen den Elementen innerhalb eines Werks beschränkt. Müsste nicht vielmehr, wiederum mit Koschorke, das Entstehen von Komplexität durch die Integriertheit der Elemente erweitert werden im Sinne einer Lockerung dieser Relationen durch Interdependenzunterbrechung resp. Erzeugung von Unbestimmtheit? ${ }^{6}$ Wenn etwas, so hat die vorangegangene Analyse genau das bestätigt. Komplexes Erzählen entfaltet seine Wirkung nicht allein durch eine Erhöhung der wechselseitigen Relationen zwischen den Elementen, also ihrer (Re-)Kombinierbarkeit, sondern durch ihre Öffnung hin auf eine Vielheit möglicher Deutungsanschlüsse. Wie anders sollte sonst die Fantasietätigkeit des Lesers angestoßen werden und wie sonst sollte er imaginativ Verbindungen zu seiner eigenen Lebenswelt herstellen können. Zudem hat sich gezeigt, dass ein Verständnis des literarischen Textes als komplexes Phänomen die Kompetenzen der literaturwissenschaftlichen Analyse im engen Sinn überschreitet, indem Einsichten aus der Komplexitätsforschung, der narrativen Ethik und den Neurowissenschaften in die Untersuchung einfließen. Genau dies wäre auch als Ermöglichungsbedingung der Hereinnahme der genannten Paradigmen in den Diskurs der Literaturwissenschaft bzw. umgekehrt des Anschlusses der Literaturwissenschaft als Teildisziplin an die Komplexitätsforschung zu verstehen. Anders käme man eben angesichts der Herausforderung durch Komplexität nicht weiter.

Bei einem Blick zurück am Ende des vorliegenden Kapitels fällt auf, dass, wie bereits angedeutet, übrigens nicht nur in der germanistischen Literarturwissenschaft bisher kaum oder gar nicht auf das Phänomen Komplexität bzw. komplexes Erzählen im engeren Sinn reagiert wurde. Bedenkt man diese

6 Vgl. A. Koschorke: »Einleitung«, in: Ders. (Hg.), Komplexität und Einfachheit (2017), S. $7 \mathrm{f}$. 
Lücke in der Beschäftigung mit dem Thema vonseiten der eigentlich zuständigen Fachdisziplin, drängen sich einem Fragen nach den möglichen Gründen dafür auf. Naheliegend ist wohl, dass die Gegenstände des forschenden Interesses zwischen einer sich textbasiert verstehenden Literaturwissenschaft und der Komplexitätsforschung, die auf eine sich dramatisch verändernde Dynamik von Natur und Gesellschaft antwortet, zu verschieden anmuten, als dass eine systematischere Annäherung zielführend auszufallen verspräche.

Etwas optimistischer stimmt hingegen der Umstand, dass die Zahl der literarischen Texte, die sich unter dem Gesichtspunkt von Komplexität analysieren lassen, langsam zunimmt. Zu nennen wären in der deutschsprachigen Literatur etwa die Romane Navid Kermanis, insbesondere Dein Name, dessen Untersuchung unter diesem Aspekt in einem späteren Kapitel ansteht. Ob die Unvorhersehbarkeit komplexer Phänomene als zu bewältigende Unüberschaubarkeit, sowohl der literarischen wie der (literatur-)wissenschaftlichen Arbeit, sich dabei eher hemmend oder, im Sinne eines Anreizes, förderlich auswirken wird, ist schwer zu sagen. Vermutlich aber wird es aus Gründen dringlicher Problembewältigung auch vonseiten der Literatur zunehmend Einlassungen auf die Herausforderung durch Komplexität brauchen. Dabei könnte womöglich die Selbstwirksamkeit der Autoren gerade in Hinsicht auf die Klimakatastrophe und ihre Eindämmung noch eine Steigerung erfahren. ${ }^{7}$ Es bliebe damit also auch für interessierte Literaturwissenschaftler in Zukunft noch viel zu tun.

7 Bis in die jüngere Zeit gibt es auch in der deutschsprachigen Cegenwartsliteratur durchaus Einlassungen auf das Thema des Klimawandels. Man denke etwa an Jakob Haslingers Phi Phi Island (2007), Illja Trojanows EisTau (2011) oder Roman Ehrlichers Malé (2020). Es handelt sich um Texte, die von der Journalistin Sieglinde Ceisel zur Climate fiction gezählt werden, einem Cenre, das durch Autoren wie George Turner (Sommer im Treibhaus 1991), Daniel Quinn (Ismael 1992), Barbara Kingsolver (Das Flugverhalten der Schmetterlinge 2012), Maja Lunde (Die Ceschichte der Bienen 2015) oder T.C. Boyle (Ein Freund der Erde 2019) bekannt geworden ist. Vgl. https://www.deutschlandfunkkultur.de/climate-fiction-co-der-klimawandel-als-rand thema-in-der.1270.de.html?dram:article_id $=458378$ vom 13.02.2021. 


\section{Der totale Roman als Vorläuferidee des komplexen Erzählens}

Steht in der etablierten Erzähltheorie der Text mit seinen Strukturen im Vordergrund, ${ }^{1}$ so verschiebt sich der Fokus, sobald dieser nur zu einem Faktor unter mehreren innerhalb des Feldes der literarischen Kommunikation wird, also zu einem Teil innerhalb eines umfassenderen, damit komplexeren Ganzen. Haben wir zuvor bereits die Wirkungen des komplexen Erzählens auf den Leser und - in komplexitätstheoretischer Hinsicht - auch die Implikationen des realen Autors (als Randbedingungen) in seinen Text am Beispiel des Romans Buenos Aires. Anderswelt erörtert, so lässt sich nun sagen, dass das komplexe Erzählen deswegen als höherstufig bzw. als emergentes Phänomen begriffen werden kann, weil es Erzählen nicht auf den manifest vorliegenden Text reduziert. Es bewegt sich damit nicht in Richtung Reduktion, sondern geht den diametral entgegengesetzten Weg. Der Begriff des komplexen Erzählens ergibt sich in unserem Sinn also erst als Auftauchen von neuen Eigenschaften auf einer höheren Ebene der Betrachtung (Emergenz) des im Vergleich mit dem individuell-einzelnen Text komplexeren Ganzen, also der Rezeption seiner einzelnen Bedeutungen sowie seines Gesamtsinns als auch seiner Entstehungsbedingungen, die sich mit der Person des realen Autors und seines Engagements in der realen Wirklichkeit verbinden lassen.

Dieser Zusammenhang ist mindestens für die frühen Romane des peruanischen Erzählers Mario Vargas Llosa nachgewiesen, ${ }^{2}$ der - neben Heimito tinez und Scheffel vertreten wird. Vgl. Matias Martinez; Michael Scheffel: Einführung in die Erzähltheorie [1999], 11. überarb. u. aktual. Aufl., München: C.H. Beck 2020.

2 Vgl. dazu das Kapitel zur Theorie des Romans in Thomas M. Scheerer: Mario Vargas Llosa - Leben und Werk. Eine Einführung, Frankfurt a.M.: Suhrkamp 1991, S. 63-85. 
von Doderer - für die eigene literarische Arbeit den Begriff des »totale[n] Ro$\operatorname{man}[\mathrm{s}] \ll,{ }^{3}$ gewissermaßen eine Vorläuferidee des komplexen Erzählens, verwendet hat. Bei einem Vergleich mit den Merkmalen komplexen Erzählens bei Mieth, wo besonders auf die Erweiterung des intellektuellen Horizonts der Leser sowie einer potentiellen Veränderung ihrer Wirklichkeitswahrnehmung abgehoben wird, und der kognitiven Implikation des Autors in seinen Text im Kybernetischen Realismus Alban Nikolai Herbsts, fällt auf, dass hier, anders herum, die Unzufriedenheit des Schriftstellers mit der ihm begegnenden Wirklichkeit als Motivation der literarischen Produktion begriffen wird. ${ }^{4}$ Der Autor des totalen Romans ist nach Vargas Llosa jemand, den die soziale Wirklichkeit, deren Ungerechtigkeit er aus eigener Anschauung durch die Herkunft aus unterprivilegierten Verhältnissen kennt, nicht befriedigt und der sich deshalb in ständigem Widerspruch zu ihr befindet. Sein Schreiben ist dabei zweierlei. Zunächst Protest gegen die schlechte, in seinen Augen verbesserungswürdige, soziale Realität. Dann jedoch auch der Versuch, im Schreiben den Gründen »für die notwendige Verarbeitung eigenen Erlebens « ${ }^{5}$ auf die Spur zu kommen, wie Thomas Scheerer schreibt, um auf diese Weise den Zwiespalt zwischen eigener Subjektivität und umgebender Realität zu bewältigen. Die als sozial engagiert begriffene Produktion hat, mit anderen Worten, Wurzeln in der Biografie des Autors. Es handelt sich dabei zumeist um »eine ganze Serie [von] Frustrationen ${ }^{6}$ im Verlauf der eigenen Sozialisation zum Schriftsteller, d.h. bei dem Versuch, das eigene Leben ganz in den Dienst der Literatur zu stellen. Ein Thema, mit dem sich Vargas Llosa auch in eigenen Untersuchungen der ihm in dieser Hinsicht nahestehender Autoren Flaubert und García Márquez beschäftigt hat. ${ }^{7}$ So geht es auch ihm darum, die Identität zu erfüllen, die ihm neben den anderen Teilidentitäten als Literaturkritiker für das Radio, Romantheoretiker, Essayist und Wissenschaftler als am meisten erstrebenswert erscheint. ${ }^{8}$

Dass man etwas tut, ohne dass man es selbst wirklich noch will, also einfach nur damit weitermacht, was man sowieso tut und zuvor schon getan hat,

3 Ebd., S. 75.

4 Vgl. ebd., S. 66 unten.

5 Ebd., S. 67.

6 Ebd., S. 68.

7 Es handelt sich um Die ewige Orgie. Flaubert und »Madame Bovary« sowie García Márquez. Historia de un deicidio.

8 Vgl. dazu Th.M. Scheerer: Vargas Llosa, S. 64 f. 
auch wenn man spürt, dass es nicht so weitergehen kann, ist das Zeichen für eine Krise. Obwohl etwas Wesentliches fehlt, das Gefühl nämlich, dass das eigene Handeln in Hinsicht auf die Zukunft Sinn hat, ist man gefangen in den Ansprüchen und Erwartungen der Gesellschaft, die, siehe Vargas Llosa, mit der eigenen materiellen Sicherung der Existenz, dem Gelderwerb zu tun haben. ${ }^{9}$ Da sich die Gesellschaft durch das Handeln ihrer Mitglieder reproduziert, und umgekehrt das einzelne Subjekt in seiner reproduktiven Aktivität, der Erwerbsarbeit, auf die Anerkennung der Gesellschaft angewiesen ist, sind die Zyklen von gesellschaftlicher und subjektiv-individueller Reproduktion so miteinander verzahnt, dass es für den Einzelnen mit einem gewissen Risiko verbunden ist, wenn er sich entscheidet, die alte Identität in Richtung auf eine neues In-der-Welt-Sein zu transzendieren. Und dies, ohne genau zu wissen, ob bzw. wie das überhaupt geht, ob also, wie im Fall Vargas Llosa, die eigene Identität sich von der eines Theoretikers hin zu einem Autor dessen, was er den totalen Roman nennt, erweitern ließe.

9 Vgl. ebd., S. 65. 



\section{Das Prinzip der alternativen Chronologien - Komplexes Erzählen in Lars Gustafssons Roman Herr Gustafsson persönlich}

Dass Identität als Emergenzphänomen des literarischen Textes, ob nun aufseiten des Lesers (als perspektivische Erweiterung bzw. Anschluss eigener lebensweltlicher Erfahrungen und damit evtl. verbundener Verhaltensänderung) oder der des Autors (im Sinne der Verarbeitung eigenen Erlebens im Schreibprozess), eine wichtige Rolle spielt, wird auch am Beispiel des schwedischen Dichter Lars Gustafsson, besonders in seinem Roman Herr Gustafsson persönlich, der als frühes Beispiel für autofiktionales Erzählen gelten kann, ${ }^{1}$ deutlich. Der Protagonist, ein junger Schriftsteller mit der Identität des realen Autors, der zudem im Erzähltext den gleichen Namen trägt, leidet in ähnlicher Weise wie Vargas Llosa an einer Wirklichkeit, die ihm sinnlos erscheint. Dies betrifft vor allem die sozialen Beziehungen, die er als kalt und unverbindlich erlebt. Er vergleicht sich deshalb mit »einem mittelalterlichen Homunkulus«, der abgeschlossen von der Wirklichkeit »in einem Glaskolben « ${ }^{2}$ überdauert. Während die Welt sich am Ende der 6oer Jahre um ihn her stark verändert, verharrt er auf seinem »Beobachtungsposten « und analysiert die gesellschaftlichen Kämpfe »[v]on der Kulisse aus «, ${ }^{3}$ jedoch ohne empathische Teilnahme. Ulrike Sander spricht in ihrer Monografie Ichverlust und fiktionaler

Zum autofiktionalen Erzählen vgl. Frank Zipfel: »Autofiktion«, in: Dieter Lamping (Hg.), Handbuch der Literarischen Gattungen, Stuttgart: Kröner 2009, S. 31-36. Außerdem Ders.: »Autofiktion. Zwischen den Grenzen von Faktualität, Fiktionalität und Literarität?«, in: Simone Winko et al. (Hg.), Grenzen der Literatur. Zu Begriff und Phänomen des Literarischen, Berlin et al.: De Gruyter 2009, S. 283-314.

2 Lars Gustafsson: Herr Gustafsson persönlich, übers. aus d. Schwedischen von Verena Reichel, Frankfurt a.M.: Fischer 1985, S. 13.

3 Ebd., S. 29. 
Selbstentwurf auch von einer »inneren >Kristallisation «.$^{4}$ Gemeint ist damit die innere Erstarrung emotionaler Persönlichkeitsanteile aus »Angst vor dem kalten vernichtenden Blick der anderen. $\ll^{5}$ Zwar ist er als Schriftsteller und Chefredakteur der Literaturzeitschrift Bonniers Litterära Magasin durchaus anerkannt und hat Kontakte zu Intellektuellen in ganz Europa wie Janos Elbert (Budapest) und Hans Magnus Enzensberger (Berlin), doch hindert ihn das Gefühl der Beliebigkeit des eigenen Handelns angesichts einer Wirklichkeit, in der es keinen teleologisch vorgeordneten Sinn mehr gibt, in der deswegen Vorstellungen von einem zu erreichenden Ziel austauschbar und willkürlich erscheinen, soziale Bindungen einzugehen.

Der Zweifel, der mit diesem Bewusstsein existenzieller Verunsicherung einher geht, reicht so tief, dass das schreibende Ich auf sich selbst zurückgeworfen wird und zunächst bei sich selbst verharrt. An diesem Punkt befindet sich der Ich-Erzähler in der Wartehalle des Frankfurter Flughafens, die er metaphorisch als »dunklen und finsteren Wald « beschreibt, in dem »der rechte Weg abhanden gekommen war. ${ }^{6}$ Wenn es einen Satz zuvor heißt: »Hier beginnt nun ein Roman. Gott weiß, wie er enden wird[...]«, so wird daran deutlich, dass die Schreib-Motivation des Autors unmittelbar aus dem Gefühl des Verirrtseins, des Nicht-weiter-Wissens resultiert. Die an diesem Punkt abbrechende Kontinuität des Subjekts, verbunden mit der tiefgreifenden Infragestellung seiner Identität, führt dazu, dass die Suchbewegung in das Medium der literarischen Erzählung übertragen und darin fortgesetzt wird. So erklärt sich, dass am Beginn der Erzählung in der Erzählung, es handelt sich hier um eine Form der komplexen Metalepse, ${ }^{7}$ der Autor wichtige Stationen seines Lebensweges, bis hin zu seiner Anstellung im Haus Bonnier, rekogniziert, um dann mit dem Erzählen des gegenwärtigen Erlebens in der Wartehalle und während des Flugs nach Berlin, wo er die unvorhergesehene Bekanntschaft der Philosophie-Dozentin Johanna Becker macht, fortzufahren.

Es zeigt sich, dass die existenzielle Orientierungslosigkeit, die sich mit dem Gefühl der Beliebigkeit eigenen Handelns verbindet und metaphorisch als Verirrtsein in einem dunklen Wald beschrieben wird, mit bisher nicht

$4 \quad$ Ulrike-Christine Sander: Ichverlust und fiktionaler Selbstentwurf. Die Romane Lars Gustafssons, Göttingen: Vandenhoeck \& Ruprecht 1998, S. 375.

Vgl. ebd.

L. Gustafsson: Herr Gustafsson persönlich, S. 11.

7 In der Typologie nach Klimek handelt es sich um eine Möbiusband-Erzählung der Kategorie 3a. Vgl. S. Klimek: Paradoxes Erzählen, S. 380-382. 
wahrgenommenen, d.h. nicht realisierten Aspekten der Emotionalität des Erzählers zu tun hat. Das Erlebnis, das ihn aus seiner emotionalen Erstarrung befreit, besteht nun überraschenderweise darin, dass er sich zum ersten Mal so wahrgenommen fühlt, wie er ist, mit all seinen bisher hinter der Fassade des erfolgreichen Intellektuellen verborgenen Ängsten und Schwächen. Die Person, die ihm das Gefühl von Geborgenheit, von menschlicher Wärme, von Anerkennung, gar Respekt und Verständnis für seine Befindlichkeit bis hin zu mütterlicher Liebe vermittelt, ist niemand anders als Johanna Becker, neben der er unbekannterweise im Flugzeug zu sitzen kommt. Dass dieser Moment des Wahrgenommenwerdens, der im gefühlten Blick des anderen auf ihn selbst besteht, ganz offenbar mit besonderer Intensität erlebt wird, kommt nicht zuletzt auch darin zum Ausdruck, dass das Verb >sehen im folgenden Zitat kursiv gedruckt ist: »Jetzt war ich gesehen [Herv. i.O.] worden; aus der tiefsten Gleichgültigkeit, aus der totalen Nichtigkeit, die für mich das natürliche und selbstverständliche Ende des Dezenniums bedeutete, war ich zu verzweifelter Aufmerksamkeit erwacht, als mich schließlich jemand angesehen hatte. $\ll^{8}$

Die Philosophie-Dozentin wird so zu seiner Anima, die ihn im Vorgang des wechselseitigen »Gesehenwerdens und Sich-sehen-Lassens « ${ }^{9}$ zum ersten Mal begreifen lässt, wer er selbst eigentlich ist und was ihm aufgrund dieser Identität zu tun aufgetragen ist. ${ }^{10}$ Während der Ich-Erzähler fasziniert »ihre

8 L. Gustafsson: Herr Gustafsson persönlich, S. 35. Ein ähnliches Phänomen findet sich übrigens in einer Erzählung von Wolfgang Hilbig. Auch in Der Heizer (1980) begegnet man einer Wahrnehmungskonstellation, bei der die Markierung des Verbs sehen in gleicher Weise wie in Gustafssons Text darauf hindeutet, dass es sich nicht um eine gewöhnliche visuelle Wahrnehmung handelt, sondern um ein mit besonderer Intensität verbundenes Erleben, das weit darüber hinausgeht. In Hilbigs Fall handelt es sich jedoch um eine innere Schau, d.h. um geistiges Sehen, eine Vorstellung also, die ich selbst in einem Aufsatz über den literarischen Text als Schauplatz der Arbeit an der eigenen Identität, versuchsweise im Sinne einer autoskopischen Halluzination nach Metzinger interpretiert habe. Vgl. André Steiner: »Eine andere Gegenwart? - Annäherungen zwischen Erzähltheorie und Neurowissenschaft«, in: Revista de Filologia Alemana 22(2014), S. 11-29, hier S. 18.

9 U.-Chr. Sander: Ichverlust, S. 188.

10 Den Vorrang des Angesehenwerdens vor dem Sehen bei der Genese des Selbstbewusstseins erläutert Hartmut Böhme am Beispiel von Sartre und Hubert Fichte: „An der Dialektik des Sehens und Angesehenwerdens entwickelt Sartre die immer prekäre Konstitution des Selbstbewußtseins. Doch ist problematisch, daß Sartre von der Situation des Sehens, nicht von der Situation des Angesehenwerdens ausgeht, also davon, 
Augen, ihre wunderbaren Augen ${ }^{11}$ bemerkt, liest er darin »etwas Ernstes, etwas, das mich aufforderte: ich solle die Welt verändern, oder zumindest ihrer Veränderung nicht entgegenarbeiten, etwas, das mir sagte, dass diese Augen nicht nur mich wahrnahmen, sondern auch meinen Platz in der Geschichte, in der Wirklichkeit [...] «. ${ }^{12}$ Wenn er im Anschluss die gesellschaftlichen Umbrüche der 6oer Jahre Revue passieren lässt - die Bekanntschaft mit Revolutionären der FNL, den Vietnamkrieg, den Beginn der Studentenbewegung in den Metropolen der westlichen Welt und die enttäuschende Rückkehr zu der »durch Gesetze geregelte[n] Ordnung ${ }^{13}{ }^{13}$ so wird über diese Beschreibung deutlich, dass seine subjektive Befindlichkeit (der emotionalen Erstarrung) im Grunde Ausdruck der gesellschaftlichen Entfremdung und der Lüge ist, die nach einer Zeit der gelebten Utopie, in welcher der Traum dabei war, Wirklichkeit zu werden, nur umso spürbarer zurückgekehrt ist. ${ }^{14}$

Zugleich mit dieser Einsicht entsteht ein Zweifel an der Fähigkeit der Sprache, die Wirklichkeit so abzubilden, wie sie ist. Sie erscheint nun, ähnlich wie bei Adorno, als Teil eines Verblendungszusammenhangs, das den Einzelnen nicht zu sich kommen und die Wirklichkeit nicht erkennbar werden lässt, sondern eher verschleiert. Der Erzähler verwendet dafür im Text die Metapher des Risses. Wenn es dort heißt: »Die Risse weiteten sich, immer größer wurde der Abstand zwischen Sprache und Wirklichkeit: da war eine Lügenmaschinerie am Werke, und die Lüge breitete sich aus wie eine Infektion und drang in alle Ecken und Ritzen ein [...], « $^{15}$ so dürfte klar sein, dass sich eine solche ideologisch verbrämte Sprache - zweckorientiert im Sinne politischer Ideologie und wissenschaftlicher Theorie oder der Sprache der Werbung in

dass ein Ich eine Situation als Wahrnehmungszentrum organisiert. Und wenn in diese egozentral strukturierte Wahrnehmungswelt ein Anderer eintritt, dann erst entsteht das Spiel von Sehen und Angesehenwerden, in der bei Sartre sich das Selbstbewußtsein bildet. Von Fichte her aber ist zu sagen: das Primäre ist nicht das Sehen, sondern das Angesehenwerden. Das ist auch das Richtigere. Die Grundsituation, was auch Jacques Lacan erkennt, ist nie der Blick, sondern das Angeblicktwerden. Dieses hat eine ambivalente Struktur: im Angeblicktwerden wird das Ich sich selbst als Gabe gegeben ebenso wie es der Verwerfung ausgesetzt werden kann [...].« Hartmut Böhme: Hubert Fichte. Riten des Autors und Leben der Literatur, Stuttgart: J.B. Metzler 1992, S. 128.

11 L. Gustafsson: Herr Gustafsson persönlich, S. 14.

12 Ebd., S. 15.

13 Ebd., S. 25.

14 Vgl. ebd., S. 21 und 25-29.

15 Ebd., S. 34. 
den audiovisuellen Medien - nicht dazu eignet, einen neuen Selbstentwurf innerhalb der literarischen Fiktion zu entfalten.

Wenn der Erzähler durch das Schreiben des Romans, der hier metaleptisch verschachtelt, strukturell genauer als Möbius-Band einer Erzählung in der Erzählung, aus den verkrusteten gesellschaftlichen Verhältnissen in Richtung auf eine neue Sinnfindung für das eigene Leben auszubrechen versucht, so ist mit dieser Veränderung der eigenen Identität, der Befreiung aus dem Gefängnis der Ich-Bezogenheit, zugleich die Suche nach einer neuen Sprache und neuen Formen des literarischen Ausdrucks verbunden. Die Bewegung des Textes ist also eine doppelte. Sie betrifft zunächst das, worum es im Roman substanziell geht, nämlich die Möglichkeit der Befreiung des eigenen Lebens von den lebensverneinenden Kräften der Stagnation und Erstarrung, die den Erzähler »auf eine Irrfahrt durch die Städte, Flugplätze und Bahnhöfe Zentraleuropas ${ }^{16}$ bis in den Turm von Cannobio am Lago Maggiore führt, wo er beginnt den Roman niederzuschreiben, den der Leser, indem er den Autor auf seiner »Wanderung zurück in die vergangenen Jahre ${ }^{17}$ bis an diesen Punkt begleitet, damit bereits gelesen hat, obwohl er doch hier gerade erst das Licht der Welt zu erblicken scheint.

Formal drückt sich diese scheinbar paradoxe Konstruktion in dem performativen Widerspruch aus, dass das Ende des Romans eben nicht nur das Ende ist, sondern zugleich der Anfang - die Beschreibung mit der die Binnenerzählung endet ist identisch mit dem Incipit des Rahmens, jene schließt nahezu bruchlos an diesen an, beide unterscheiden sich nur durch die typologische Markierung kursiv/recte und die Anführungszeichen, die den Text im Text fassen und damit den metaleptischen Sprung zwischen den identischen, letzten und zugleich ersten Sätzen des Romans erkennbar werden lässt. ${ }^{18}$ Aufschlussreich ist nun, dass die metaleptische Figur der Möbiusband-Erzählung nicht nur als gewissermaßen technische Anweisung an den Leser zu verstehen ist, den Text mehr als nur ein einziges Mal lesend zu durchlaufen, sondern zugleich die »Nullstelle des Diskurses ${ }^{19}{ }^{19}$ also die Umstände seiner Entste-

16 Ebd., S. 134.

17 Ebd., S. 65.

18 Die Sätze, über die das Ende mit dem Anfang rekurrent gesetzt wird, lauten: „Über den Flußtälern von Rhein und Main lag die blaugraue Schicht der schwefeldioxydfarbenen Luft wie ein Deckel. Frankfurts chemische Industrie mischte eine tückische kleine Dosis von tödlichem Gift in die Luft. «Ebd., S. 169 und 9.

19 Wolfgang Iser: Emergenz. Nachgelassene und verstreut publizierte Essays, hrsg. von Alexander Schmitz, Konstanz: University Press 2013, S. 42. 
hung, ${ }^{20}$ markiert. Damit ist dessen Ermöglichung, die eigentlich außerhalb seiner selbst liegt, sprich beim Autor resp. Herausgeber, in den Diskurs selbst hineingezogen und wird durch das Mise en abyme der Erzählung in der Erzählung erst sichtbar. Die Migration der Nullstelle in den Diskurs (der Erzählung) hat nun, wie Wolfgang Iser in seinen Ausgangsüberlegungen zum Paradigma der Emergenz schreibt, eine »Zerstörung des Textgewebes« zur Folge, die »Textualität von ihrer als selbstverständlich vorausgesetzten Trägerschaft für anderes« befreit und damit »als Ermöglichung jedweder Darstellung « ${ }^{21}$ erst wahrnehmbar macht.

Nimmt man den Aspekt der Begründung resp. Legitimierung des Schreibens, verstanden im Sinne des Hervorbringens von (Erzähl-)Diskursen, mit hinzu, so gilt es, das, was historisch durch die Herausgeberfiktion an "Autorität für die Darstellung erborgt wird «, ${ }^{22}$ nun aus dem Verhältnis des realen Autors zu bestimmten Strukturen der von ihm hervorgebrachten Narration, besonders dem Erzähler, zu gewinnen. Tritt der Autor selbst in die Legitimierungsfunktion der auktorialen Instanz ein, so werden also die von ihm generierten Erzählerfiguren in der Weise operationalisierbar, dass sie das erzählte Geschehen in je spezifischer Weise auslegen, es perspektivieren, um es dem Leser zu realisierendem Nachvollzug anzubieten. ${ }^{23}$ Ist nun die Identität zwischen textexternem, realem Autor und der Figur des (Ich-)Erzählers in der Narration, wie im Fall des vorliegenden Romans von Gustafsson, gewollt identisch, so wird sie zum Gegenstand eines Spiels zwischen beiden Komponenten, wird als fixe Idee einer unveränderlichen Substanz, eines sich immer gleich bleibenden Persönlichkeitskerns abgelöst vom Prozess des beständigen Hervorbringens, für den hier das Schreiben des Romans als Synekdoche

20 Der Ich-Erzähler beginnt mit der Niederschrift Ende Februar 1970 in einem Turm bei Cannobio, einem Dorf im Herzogtum Novarra (vgl. L. Gustafsson: Herr Gustafsson persönlich, S. 85 f. u. 169) und endet im Juli des gleichen Jahres in Väster Våla (vgl. ebd., S. 160), einem kleinen Ort in Västmanland, östlich des Sees Åmmänigen unweit von Västeras, der Geburtsstadt des Schriftstellers Lars Gustafsson.

21 W. Iser: Emergenz, S. 43.

22 Ebd., S. 42.

23 Vgl. ebd. Ich referiere hier auf Isers Darstellung, um zu zeigen, auf welche Weise die verschiedenen Komponenten des literarischen Textes (Autor-Erzähler-Leser) im Modus der Rückkopplung, die eine Modalität von Emergenz ist, zusammenwirken. Modalitäten bzw. Veranlassungen von Emergenz sind nach Iser: 1. Generativer Einschnitt, 2. Rekursivität, 3. Rückläufige Kausalität, 4. Mehrstelligkeit, 5. Nullstelle. Vgl. ebd., S. 43. Außerdem ist dort jeder Modalität ein eigenes Kapitel gewidmet. 
steht, in dem Identität als narrative, sich beständig (re-)produzierende gedacht wird.

Damit die Frage nach Identität, ihrer transitorischen Veränderbarkeit, als emergentes Phänomen der textuellen Darstellung deutbar wird, kommt es hier besonders darauf an, die Rückkopplungsstruktur des Textes nicht nur einfach als simple metaleptische Grenzüberschreitung aufzufassen, sondern sie operativ hin zur Möglichkeit alternativer Chronologien des Erzählens der gleichen bzw. einer ähnlichen, diesmal in die Zukunft entworfenen Geschichte zu erweitern - wiederum ein Paradox, denn die neuerliche (Re-)Produktion/Lektüre stünde in diesem Fall ja noch bevor! ${ }^{24}$ Die rückgekoppelte Struktur des Textes insgesamt ließe sich dann nämlich so verstehen, dass der Output der Bemühungen des Erzählers in Form der letzten Zeilen des Romans Herr Gustafsson persönlich als neuer Input zu einer Reécriture wie Relecture fungieren könnte, zumindest was die Darstellung gewisser Episoden betrifft, in denen das Gefühl der Beliebigkeit eigenen Handelns vorherrschend war. Die Zerstörung des Textgewebes, von der Iser spricht, ist somit der Preis für die Aktivierung der Nullstelle im Raum des Diskurses selbst. Was an seine Stelle tritt, ist nichts anderes als die Idee des kybernetischen Regelkreises und seine Übertragung auf das literarische Erzählen, das damit einer Logik nachträglicher Kausalität zu folgen beginnt, wie sie auch für die Konstruktion von personaler Identität in Akten der Narration (narrative Identität) charakteristisch ist.

Wie Iser weiter schreibt, entstehen durch die Interaktion von Input und Output »Kombinationsverhältnisse, die sich in Kaskaden von Möglichkeiten entladen. $\aleph^{25}$ Man kann sich vorstellen, dass dadurch, wie erörtert, eine Ver-

Die Idee der alternativen Chronologien des Erzählens ist angelehnt an das Konzept der stimmigen Geschichten von Griffith aus dem Roman Les particules élémentaires (dt. Elementarteilchen) von Michel Houellebecq. Wie Thomas Klinkert in seiner Monographie Epistemologische Fiktionen gezeigt hat, handelt es sich dabei um eine Transposition der Heisenbergschen Unschärferelation auf die erinnernde Rekonstruktion eines Lebens durch Michel Djerzinski, eine der Hauptfiguren des Romans. Vgl. Thomas Klinkert: Epistemologische Fiktionen. Zur Interferenz von Literatur und Wissenschaft seit der Aufklärung, Berlin: De Gruyter 2010, S. 326-328. Auch in diesem Zusammenhang geht es darum, dass es im (erzählten) Erinnern keine eindeutige Referenzbeziehung auf das erinnerte Geschehen gibt. Anstelle der einen Geschichte, die definitiv wahr wäre, tritt eine Vielzahl von möglichen Versionen, die, analog zur hypothetischen Geschichte eines Elektrons, dessen Parameter aufgrund der beobachterabhängigen Unschärfe nicht genauer bestimmbar sind, als »plausible, konsistente Hypothesen « eines Lebens fungieren. Ebd., S. 328. 
flüssigung der Vorgaben des Textes eintritt, die sich besonders auf die Rollenidentität übertragen lässt, die als eigene vom Erzähler ohnedies als erstarrt, lebensfern und deswegen veränderungsbedürftig kritisiert wird. Denkt man nun die Überlagerung der Input-Output-Vorgänge hinzu, weil viele von ihnen simultan ablaufen - im Kontext kultureller Selbsthervorbringung (des Menschen) gibt es kein geregeltes Nacheinander wie in der Regelungstechnik $^{26}$-, so dürften sich auf diese Weise bei einer Relektüre des gleichen Textes im zeitlich gewandelten Kontext einer Leserbiografie zu einem späteren Zeitpunkt (potenziell) durchaus andere Lesarten, ja möglicherweise alternative Chronologien des erzählten Geschehens ergeben. ${ }^{27}$ Diese Praxis ist auch bereits in der Struktur des Romans angelegt, insofern dieser aus zahlreichen Fragmenten der Erinnerung des Erzählers mosaikartig zusammengesetzt ist, und nur im Groben einer linearen Chronologie folgt, die im Erzählen bis an den Gegenwartspunkt der erzählten Welt führt, den Beginn der Niederschrift des Romans, um dann neuerlich in die Erinnerung einzutauchen. Insgesamt beruht die Logik der alternativen Chronologien darauf, dass der Leser sich, möglicherweise auch bereits während des ersten Lektüredurchlaufs, ganz sicher aber im zeitlichen Verlauf einer ganzen Leserbiografie, in vielerlei Hinsicht, was das Wissen, die Erfahrung, das ästhetische Interesse betrifft, verändert und dass diese Wandlungen wiederum zurückwirken auf die Interaktion mit dem Text. Dies kann in der Weise geschehen, dass Lektüre-Ereignisse plötzlich in anderem Licht erscheinen, deswegen anders rekontextualisiert

26 Vgl. ebd., S. 79.

27 Produktionsästhetisch gewendet findet sich das Phänomen der alternativen Chronologie im Sinn von multiplen Ereignisverläufen auch im neueren Film. Ein Beispiel dafür ist VANTACE PoINT, dt. 8 BlickWIN Kel (USA 2007, R: Pete Travis). Der Plot des Films, ein Attentat auf den amerikanischen Präsidenten während eines Anti-Terror-Gipfels im spanischen Salamanca, wird aus acht verschiedenen Perspektiven (Blickwinkel) dargestellt. Je Blickwinkel wird eine andere Chronologie des Ceschehens präsentiert, die sich mehr oder weniger simultan zu den übrigen ereignet. Nach jedem Durchlauf findet eine Zeitumkehr statt und das Geschehen beginnt mit einem neuen Loop, diesmal aus einer anderen Perspektive, von vorn. Zusammengenommen generiert der Zuschauer aus den verschiedenen Perspektiven auf das Ereignis eine Synthese des Cesamtgeschehens. Dies wäre für eine einzige Chronologie zu komplex, wäre darin nicht unterzubringen. Erst aus der Zusammenschau der Vielzahl der Varianten ergibt sich für den Rezipienten die Möglichkeit den Überblick, den sogenannten Vantage point, zu generieren. Vgl. dazu A. Steiner: »Filmisch inszenierte Simultaneität«, in: Hubmann/Huss (Hg.), Simultaneität (2013), S. 386-394. 
bzw. neu bewertet werden müssen oder vielleicht sogar aus dem Bewusstsein verschwinden, wenn auf einmal neue Zusammenhänge in den Fokus der Aufmerksamkeit rücken. ${ }^{28}$

28 Hier wird bereits deutlich, dass die Wirklichkeit im Roman nicht mehr begriffen wird als mimetische Darstellung einer Folge von Ereignissen, die einmal so geschehen und nachträglich nicht veränderbar sind. Anstelle dieser Auffassung tritt die Vorstellung, dass das Wirkliche durch Modelle ersetzt wird, Modelle, die darüber entscheiden, was als wirklich gilt und was nicht. Wenn, wie in Lars Gustafssons Roman, der manifeste Text mit sich selbst rückgekoppelt wird, so handelt es sich dabei um ein solches Modell von Wirklichkeit. Die dazugehörige Theorie der Simulation hat Jean Baudrillard in Der symbolische Tausch und der Tod [1976] hergeleitet. So ist auch die Idee der alternativen Chronologie im Sinne eines Modells (aufseiten der Rezeption) zu verstehen, das sich im Rahmen seiner simulativen Evaluation durch den Leser realisiert, wobei sowohl die Daten des Textes als auch der aktuellen Weltwahrnehmung in den Syntheseprozess einfließen. Damit wiederum korrespondiert der Begriff des »Erlebnismodell[s]«, den Vilém Flusser in Anlehnung an die Modelle der Simulation als eigentlichen Zweck literarischer Texte in die Diskussion gebracht hat: »Dichten ist das Herstellen von Erlebnismodellen, und ohne solche Modelle würden wir beinahe nichts wahrnehmen können. Wir wären anästhesiert und würden - auf unsere verkümmerten Instinkte angewiesen-blind, taub und gefühllos herumtorkeln müssen. Die Dichter sind unsere Wahrnehmungsorgane.« V. Flusser: Die Schrift, S. 66. Auf das Konzept der Simulation wird später noch im Zusammenhang der Erörterung von Echtzeit und literarischem Erzählen am Beispiel des Romans Dein Name von Navid Kermani zurückzukommen sein.

Hier soll darauf hingewiesen werden, dass der zugrundeliegende theoretische Ansatz bereits in der Erzählung Die Berliner Simulation von Bodo Morshäuser Berücksichtigung gefunden hat. Auch dort geht es exakt um den erörterten Zusammenhang der Substitution unmittelbar erlebter Wirklichkeit durch Modelle der Simulation. So heißt es im Text: »Nicht die Ereignisse, sondern die Modelle werden wiederholt. Längst dominieren sie, was passiert. Die Emotionen sind festgelegt auf jeweils zwei Möglichkeiten. In diesen Modellen sollen wir bleiben wie in einem Hamsterrad, denn in ihnen bleibt nichts wirklich, nur die Simulation.«Bodo Morshäuser: Die Berliner Simulation [1983], Berlin: Berliner Zeitung/Paperview 2007, S. 78. Auch das Subjekt ist darin bereits weitgehend dekonstruiert, wenn im Weiteren davon die Rede ist, »daß es Zeugnis ausgelaugten abendländischen Denkens sei, die Kategorie des Zentrums zu bemühen, es gebe nur noch Schaltkreise und Fangnetze.«Ebd., S. 99. Das Subjekt als Konvergenzzentrum, wie es etwa auch in der Neurobiologie heuristisch angenommen wurde, scheint damit verabschiedet. Es erweist sich nunmehr lediglich als Produkt von Modellen, Konstrukten bzw. Diskursen, in denen es erst entsteht und hat somit keine eigene Substanz mehr. Dies wird auch deutlich an einer Stelle der Narration, wo der Ich-Erzähler vergeblich versucht, einen Brief an seine Geliebte Charlot zu schreiben. Angesichts der unüberschaubaren Pluralität von Möglichkeiten des kommunikativen Ausdrucks ge- 
Einmal mehr »erweist sich Rückkopplung als Modalität von Emergenz «, ${ }^{29}$ wie bereits Iser festgestellt hat. Es wundert daher nicht, dass er bereits in seinem frühen, klassisch gewordenen Hauptwerk aus den 70er Jahren Der Akt des Lesens das Verhältnis zwischen Leser und Text in genau dieser Weise beschrieben hat, nämlich im Sinne einer kybernetischen Rückkopplung, die sich in seinen Worten auch als »Servomechanismus ${ }^{30}$ fassen lässt. Der literarische Text »verkörpert demnach ein Inventar von impulssetzenden Zeichen (Signifikanten)«, die im Lektüre-Prozess eine ständige Rückwirkung im Leser auslösen, derart, dass dieser "seine Vorstellungen in den Wirkungsvorgang eingibt. ${ }^{31}$ Ein solches Feedback hat nicht nur den Sinn, dass die mit der Denotation sprachlicher Zeichen verbundene Unvorhersagbarkeit austarierbar wird - ich kann im Vorhinein nicht wissen, was ein Text bedeutet, dies ist erst nach seiner Lektüre möglich -, sondern zudem auch die Korrektur eines zunächst vom Leser selbst abweichend gebildeten Signifikats ermöglicht. ${ }^{32}$ Die Bildung alternativer Chronologien durch den Leser ließe sich dann auch so erweitern, dass die Relektüre nicht mehr linear im strengen Sinn verlaufen muss, sondern spontan irgendwo im Text beginnen kann, wo er zufällig aufgeschlagen wird. Auf diese Weise wird die Aufmerksamkeit auf dekontextualisierte Passagen des Textes gelenkt, die dann möglicherweise anders desambiguiert werden als bei einer streng linearen (Erst-)Lektüre, die vorn beginnt und am Schluss endet, ohne sich ein Vor- bzw. Zurückspringen zu erlauben. ${ }^{33}$

lingt es ihm nicht, seine Intention mit der Handlung der Notation zu synchronisieren. Er blickt bereits aus der Perspektive der Nachträglichkeit auf sein gescheitertes Unterfangen, als sei das Briefeschreiben mit Papier und Stift noch vor Beginn der Epoche allgemeiner Digitalisierung bereits ein Anachronismus geworden, sodass es zu keiner Realisation mehr kommen kann: »lch kann keinen Brief an Charlot formulieren. [...] Die vielen Auslegungsmöglichkeiten verursachen ein Schwindelgefühl, und quer übers Blatt ziehe ich den Schlußstrich, zum xten Mal. Ich mache den Fehler, an die lesende Charlot zu denken, während ich ihr einen Brief schreibe. Ich möchte genau sein, aber es gibt keine Genauigkeit in den Prozessen. Genauigkeit ist im besten Fall genau einen Augenblick zu spät.«Ebd., S. 24.

W. Iser, Emergenz, S. 78.

30 Wolfgang Iser: Der Akt des Lesens. Theorie ästhetischer Wirkung. 4. Aufl. München: Fink 1994, S. 111.

31 Ebd., S. 110.

32 Ebd., S. $110 \mathrm{f}$.

33 In analoger Weise spricht auch Erika Fischer-Lichte davon, dass die von jeder Art der kausalen Verkettung befreiten Elemente einer Aufführung nur noch bedeuten, was sie 
Entsprechend zu der von Siegfried J. Schmidt im Rahmen der Empirischen Literaturwissenschaft (ETL) vorgeschlagenen Kategorie Polyvalenz ergeben sich bei der Rezeption literarischer Texte nicht nur in semantischer Hinsicht »unüblich komplexe kognitive Operationen«. Es erweist sich zudem im sozialen Vergleich, wie Schmidt weiter schreibt, »daß der Ergebnistyp kognitiver Operationen angesichts literarischer Phänomene unvorhersehbar ist. $^{34}$ Das Phänomen unvorhersehbarer Ereignisverläufe aus der Theorie komplexer Systeme findet sich also im vorliegenden $\mathrm{Zu}$ sammenhang modifiziert durchaus wieder (nicht nur als abweichende oder alternative Chronologie!). Dies versteht sich insofern, als die Ergebnisse der biologischen Kognitionsforschung belegen, dass Sinn und Bedeutung nicht einfach als Wahrnehmungsangebote literarischen Texten entnommen werden können, da »Wahrnehmung ein konstruktiver Prozess und kein >die Wirklichkeit repräsentierender Akt ist ${ }^{35}$ Dies bedeutet einmal mehr, dass der Leser mit seiner individuellen Identität »als empirische Instanz der Sinnkonstruktion ${ }^{36}$ viel stärker berücksichtigt werden muss. So sind es Prozesse der Selbstorganisation (selbstreferenzielle Operationen), aus denen Sinn und Bedeutung anlässlich der Auseinandersetzung mit dem literarischen Text im kognitiven System des Rezipienten emergieren. Es verwundert dann kaum noch, dass diese von Leser zu Leser unterschiedlich verlaufen (können).

selbst sind, auf diese Weise selbstreferenziell werden und gerade dadurch »im wahrnehmenden Subjekt eine Fülle von Assoziationen, Vorstellungen, Gedanken, Erinnerungen, Gefühlen hervorzurufen vermögen und ihm Möglichkeiten eröffnen, sie zu anderen Phänomenen in Beziehung zu setzen. «Erika Fischer-Lichte: Ästhetik des Performativen, Frankfurt a.M.: Suhrkamp 2004, S. 243. 



\section{Kybernetische Rückkopplung und Erinnerung in Marcel Prousts Recherche}

Denkt man nun vom Diskurs literarischen Erzählens weiter zum eng damit verbundenen Erinnerungsdiskurs, so fragt sich, inwiefern auch für diesen das Prinzip kybernetischer Rückkopplung relevant sein mag. Wenn, wie Iser betont, die unausgesetzte Veränderung aller menschlichen Lebensbereiche durch Emergenz bewirkt wird, das Emergente somit zu einer Universalie wird, ${ }^{1}$ so liegt es nahe, dies auch für den Prozess des Erinnerns zu reklamieren, sofern es sich dabei um ein erneutes Hervorbringen, i. e. die nachträgliche Bearbeitung eines bereits Erlebten, handelt, und nicht um eine Identifikation mit gesetzten Inhalten. $\mathrm{Zu}$ berücksichtigen ist dabei wohlgemerkt wiederum, »daß sich«, wie Iser schreibt,

»[...] das Emergente niemals an sich, sondern immer nur im Verbund mit etwas anderem fassen läßt. Eine solche Gebundenheit konstituiert dann auch den Cestaltwandel des Emergenten, das in seinen Operationen von dem bedingt ist, worin es sich auswirkt. Es wird daher unterschiedliche Modalitäten des Hervorbringens geben, je nach dem Bereich, in dem das Emergente zur Erscheinung kommt. $\ll^{2}$

$\mathrm{Da}$ es sich in unserem Fall um kulturelle, genauer gesagt literarische Prozesse von Selbsthervorbringung handelt, worin das Erinnern eine zentrale Rolle spielt, wird sich auch Emergenz in spezifischer Weise der Modalitäten bedienen, die für den genannten Diskurskontext grundlegend sind. Diese wären, wie bereits erläutert, rekursive Rückkopplung, rückläufige bzw. nachträgliche

1 Vgl. W. Iser: Emergenz, S. 44.

2 Ebd., S. 37. 
Kausalität, Einführung der Nullstelle in den Diskurs sowie Mehrstelligkeit, die als Begriff am literarischen Beispiel noch erörtert werden muss.

Nun ist für den genannten Zusammenhang, zumindest für die Literaturgeschichte der Moderne, das bekannteste Beispiel sicher das MadeleineErlebnis des Ich-Erzählers Marcel aus dem ersten Band der Proustschen Recherche. Wenn Iser Emergenz ganz allgemein als Modalität fasst, »wie etwas in die Welt kommt [...], das es bisher nicht gab «, ${ }^{3}$ so kann man darin den Versuch sehen, der unerklärlichen Grundlosigkeit des Daseins etwas entgegenzusetzen, das den entzogenen Grund, die unzugängliche »Ursprungswirklichkeit « ${ }^{4}$ nachträglich besetzt. Eine solche »retroaktive Besetzung der Grundlosigkeit «, ${ }^{5}$ die Iser generell für den Mythos und seine immer fortgesetzte Bearbeitung zugrunde legt, ${ }^{6}$ lässt sich indessen auch für den Erinnerungsvorgang sinnvoll adaptieren, sofern man darin eine nicht abschließbare »rückwärts gewendete Besetzung des Entzogenen « ${ }^{7}$ erkennen mag. So bleibt das, was potenziell im Akt des Erinnerns rekogniziert werden kann, solange durch das Vergessen dem Bewusstsein entzogen, bis es im Prozess senso-materieller Vergegenwärtigung von neuem zum Leben erweckt wird. Diesen Vorgang der Instantiierung des Vergangenen ist in Prousts Recherche mit dem Madeleine-Erlebnis verknüpft. Indem der Erzähler Marcel den sensuellen Reiz des Geschmacks der zusammen mit einem Schluck Tee eingenommenen Madeleine wahrnimmt, wird ein Strom innerer Bilder getriggert, der ihn zurück in seine Kindheit nach Combray führt. Somit stellt der Geschmack der Madeleine, dessen Bekanntschaft er einst durch seine Tante Léonie gemacht hatte, den Schlüsselreiz dar, der ihm ganz unerwartet, daher eher zufällig und nun auf Vorschlag seiner Mutter durch den neuerlichen Genuss einer Madeleine, Einlass in »das unermeßliche Gebäude der Erinnerung « ${ }^{8}$ gewährt.

Hier nun besteht die Möglichkeit, den Erinnerungsvorgang, wie er von Proust geschildert wird, zumindest in seiner Form als Mémoire involontaire, in komplexitätstheoretischer Hinsicht als emergentes Phänomen aufzufassen, indem nämlich der ursprüngliche Reiz des Madeleine-Geschmacks im

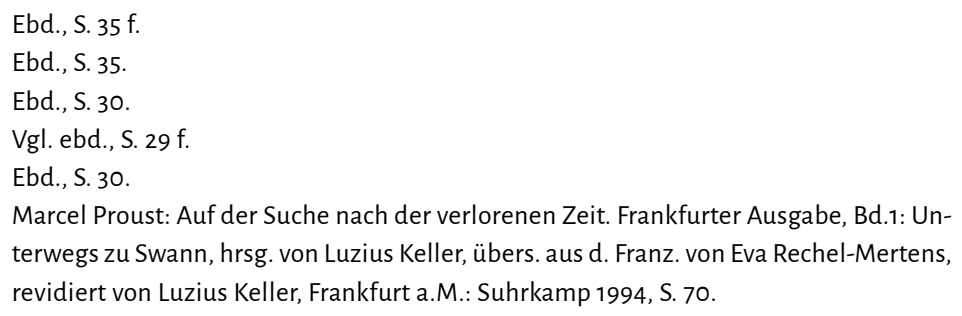
terwegs zu Swann, hrsg. von Luzius Keller, übers. aus d. Franz. von Eva Rechel-Mertens, revidiert von Luzius Keller, Frankfurt a.M.: Suhrkamp 1994, S. 70. 
Sinne des Outputs (einer vergangenen, gustatorischen Sensation) nunmehr als Input und damit Initial einer Erinnerungskaskade im szenisch-autobiografischen Gedächtnis fungiert, die am Anfang einer umfassenden Rekursion steht, in deren Verlauf die verloren geglaubte Welt der Kindheit in ihrer Totalität von neuem ersteht. ${ }^{9}$ Vergegenwärtigt man sich zudem, dass der IchErzähler sich am Ende des Romanzyklus der Recherche anschickt, genau jenen Roman niederzuschreiben, ${ }^{10}$ den der Leser, ist er ihm bis an diesen Punkt ge-

Als Schlüsselreiz fungieren dabei nicht nur der Ceschmack der Madeleine, sondern auch das Geräusch eines Löffels sowie die taktile Wahrnehmung der Unebenheit von Bodenplatten. Wie im folgenden Textausschnitt deutlich wird, geht es dabei um die Korrespondenz zwischen aktueller Gegenwartswahrnehmung und erlebter Vergangenheit, die sich auch als Input-Output-Relation der daran gekoppelten Erinnerungskaskade verstehen lässt. Dieses Erleben ist zudem verbunden mit dem, was man als Erweiterung der Identität des Erzählers bezeichnen kann, die sich gewissermaßen über die Zeit erhebt und dabei erfährt, dass die Zeit ihr nichts anhaben kann: »Ich glitt sehr rasch über all das hinweg, denn weit zwingender rief mich die Aufgabe, den Grund jenes Clücks, jener Art von Cewißheit zu suchen, mit der sie sich aufdrängte, eine Suche, die ich früher stets verschoben hatte. Dieser Grund nun begann sich mir zu offenbaren, wenn ich jene beseligenden Eindrücke untereinander verglich, denn sie hatten untereinander gemeinsam, daß ich das Geräusch des Löffels an dem Teller, die Ungleichheit der Bodenplatten oder den Ceschmack der Madeleine zugleich im gegenwärtigen Augenblick und in einem entfernten Augenblick wahrnahm, und zwar in einem Maße, daß die Vergangenheit auf die Gegenwart übergriff und ich nicht mehr mit Bestimmtheit wußte, in welcher von beiden ich mich befand; in Tat und Wahrheit war es so: Das Wesen, das dann in mir diesen beglückenden Eindruck empfand, empfand ihn darin, was dieser zu einem früheren Zeitpunkt und jetzt an Cemeinsamem hatte, darin, was er an Außerzeitlichem hatte; es war ein Wesen, das nur dann in Erscheinung trat, wenn es aufgrund einer solchen Identität zwischen Cegenwart und Vergangenheit in das einzige Lebenselement versetzt wurde, in dem es existieren und die Essenz der Dinge genießen konnte, das heißt außerhalb der Zeit. [...] Als einziges hatte es die Macht, mich die früheren Tage, die verlorene Zeit, wiederfinden zu lassen, während die Bemühungen meines Cedächtnisses und meines Verstandes dabei immer scheiterten.« M. Proust: Suche nach der verlorenen Zeit, Bd. 7: Die wiedergefundene Zeit, S. 264-266.

Auch wenn im Fall von Proust der Beginn der Niederschrift nicht Teil der Erzählung ist, so steht er doch kurz davor, mit ihr zu beginnen. Vgl. ebd., S. $516 \mathrm{f}$. Körperlich und seelisch geschwächt und von der »Idee des Todes « (ebd., S. 519) heimgesucht ist er sich jedoch unsicher, ob er noch dazu imstande sein wird. Dann stellt er sich vor, als wäre es bereits vollbracht, wie er ans Werk geht: »Was ich selbst zu schreiben hatte, war anderes und längeres, und es richtete sich auch an mehr als nur eine Person. Ja, es war lang, was ich zu schreiben hatte. Am Tag würde ich höchstens versuchen können zu schlafen. Wenn ich arbeitete, würde es nur nachts geschehen können. Doch es würde 
folgt, in der nachvollziehenden Lektüre bereits antizipiert hat, so ergibt sich daraus eine Anschlussmöglichkeit, die über den Modus nachträglicher Kausalität auf den strukturell vergleichbaren Text von Gustafsson verweist. Hier wie dort geht es um eine rekursiv rückgekoppelte Struktur des Erzählens, die sich in der Rezeption, also bei der Rekonstruktionsarbeit des Lesers, in der beschriebenen Weise auswirkt.

Aufgegeben ist bei Gustafsson allerdings der von Proust gewiss weitgehend eingelöste Anspruch totaler Reanimation von Vergangenheit im Innenraum des erzählenden Subjekts, zusammen mit der Darstellung der »Gesetzmäßigkeiten, unter denen das Vergangene wieder gegenwärtig werden kann. «" An seiner Stelle findet sich bei Gustafsson nicht mehr das initiale, mit großen Glücksgefühlen verbundene Erlebnis der in der Erinnerung wiedergefundenen Zeit, das einem widerfährt bzw. geschenkt wird. Als Motivation des schreibenden Subjekts tritt hier die Suche nach der Wahrheit eines bisher nicht gelebten Lebens und den nicht eingelösten Möglichkeiten gesellschaftlicher Veränderung. Der damit verbundene Affekt ist nicht der des Glücks, sondern der der Trauer. Das Schreiben bekommt für den Ich-Erzähler die Bedeutung, die in der Psychologie auch als Trauerarbeit bezeichnet wird. ${ }^{12}$ Sie kommt mit der Niederschrift des Romans insofern an ein Ende, als damit die Vergangenheit des scheibenden Subjekts ästhetisch durchgearbeitet und gestaltet vorliegt und mit der Veröffentlichung, die selbst freilich nicht mehr zur Histoire des Romans gehört, Teil des gesellschaftlichen Prozesses der Aufarbeitung der im Text verhandelten Periode bis zum Anfang der 7oer Jahre wird.

vieler Nächte bedürfen, vielleicht hundert, vielleicht tausend. Ich aber würde in der Angst leben, nicht zu wissen, ob der Herr meines Ceschicks, der weniger nachsichtig als Sultan Scheriar war, am Morgen, wenn ich meine Erzählung unterbrach, mein Todesurteil noch etwas aufschieben und mir erlauben würde, am nächsten Abend darin fortzufahren.«Ebd., S. $520 \mathrm{f}$.

Der Brockhaus Literatur: Schriftsteller, Werke, Epochen, Sachbegriffe, 2. völlig neu bearb. Aufl., hrsg. von der Lexikonredaktion des Verlags F.A. Brockhaus, Leipzig et al.: Brockhaus 2004, S. 656, hier Lemma »Proust, Auf der Suche nach der verlorenen Zeit«.

12 Vgl. dazu auch L. Gustafsson: Herr Gustafsson persönlich, S. 44 und 169. 


\section{Mehrstelligkeit und rückläufige Kausalität als Modi der komplexen Erzählung}

Kehren wir mit der Absicht, die bis hierhin am Beispiel der Werke von Gustafsson und Proust erläuterten Modalitäten von Emergenz auch für Alban Nikolai Herbsts Anderswelt-Roman Buenos Aires zu ergänzen und bei dieser Gelegenheit auch den noch ausstehenden Term Mehrstelligkeit einzuführen, noch einmal zurück an den Anfang der Untersuchung. Wenn Iser Recht hat mit der These, dass »kybernetische Schleifen [...] die Infrastruktur gegenwärtiger Welt [bilden] «, ${ }^{1}$ so lässt sich dies mit Mieth, sofern komplexes Erzählen die (über-)komplex gewordene Lebenswirklichkeit widerpiegeln solle, ${ }^{2}$ auf fiktionale Wirklichkeit und Figurenidentität erweitern.

Wie gesehen, gibt es in Herbsts Roman Austauschbeziehungen rekursiver Natur zwischen den künstlich programmierten Welten (W1 bzw. W2) und der Simulation Garrafff (W3), die aus Sicht des Programmierers Herbst wirklich zu sein scheint, weil er auf dieser Ebene realiter als Angestellter der Firma Cybergen beschäftigt ist. Die Rückkopplung wird sprachlich über den Wechsel der Personalpronomen angezeigt (von der 3. zur 1. Person und wieder zurück), der semantisch als Vermengung bzw. Wechsel der Figuren-Identitäten von Herbst und Deters verstanden werden kann. Erzähltheoretisch könnte man das Phänomen auch so beschreiben, dass es zu einer metaleptischen Überschreitung der erzählten Welten kommt, deren Schnittstellen allerdings aus der Bewusstseins-Perspektive des Ich-Erzählers Herbst wahrgenommen (Mitsicht) und nicht denotativ im Text markiert werden. Es kommt somit zu einer rekursiven Rückkopplung zwischen programmierter Figur und Programmierer im Bewusstsein des Erzählers, der zugleich der Programmierer ist. Dies realisiert sich in Form einer Überlagerung der Input-Output-

1 W. Iser: Emergenz, S. 82.

2 Vgl. D. Mieth: Moral und Erfahrung, S. 102. 
Vorgänge, die quasi simultan ablaufen, wie am Beispiel der Leser-Interaktion in Gustafssons Möbiusband-Erzählung bereits aufgezeigt. Aufschlussreich ist hier nun, dass Schreiben und Lesen, Produktion und Reproduktion, nicht mehr eindeutig den Funktionen von Autor (textextern oder -intern) und Leser (textextern) zugeordnet werden können, sondern das Schreiben, sobald es sich in einem technischen Sinn interaktiv realisiert, selbst Teil von informatischer Wirklichkeit wird, deren Modus der Regelkreis ist. Wenn, wie im vorliegenden Kontext der Programmierung simulierter Welten, das Programmieren zur Semantik des Verbs >schreiben dazugehört, denn dies ist genau die Aktivität, mit der Herbst als Alter ego und `Quelle` des Protagonisten Hans Erich Deters befasst ist, dann steht >programmieren metonymisch auch für das literarische Schreiben. Doch umgekehrt realisiert in einem technischen Sinn das computergestützte, informatische Netzwerk, in dem die Programme die künstlichen Welten, sowohl des Romans als auch der (virtuellen) Realität (im Allgemeinen), ja erst generieren, die Funktion, die textextern vom Rezipienten mithilfe seiner Imagination wahrgenommen wird.

Rückläufige Kausalität ist hier somit auf eine andere, letztlich sogar zweifach andere Weise als bei Gustafsson im Text wirksam. Diesmal nicht dadurch, dass der Weg zum Ziel des Erzählbeginns narrativ entfaltet wird, also die Voraussetzungen/Ursachen, die in der Person des textexternen wie intradiegetischen Autor-Erzählers liegen, zum Gegenstand des Erzählens gemacht werden, sondern zunächst dadurch, dass der Erzähler-Programmierer Herbst versucht, die kybernetischen Operationen auf sich selbst anzuwenden, die zur Existenz seines Avatars Deters geführt haben, indem er sich aus W3 löscht, in W1 an die Stelle von Deters kopiert und auf diese Weise wieder eins, also eine homogene Identität zu werden versucht, was aus den genannten Gründen freilich scheitert. Damit ist gemeint, dies sei hier nochmals mit Herbsts eigenen Worten betont, der »Kontaktverlust $\aleph^{3} \mathrm{zu}$ eigenen Lebensfunktionen, zu sich selbst, verstanden als autonomes Individuum. Ein simpler Copy-andpaste Befehl, dessen Bedeutung jedoch einen »Hof « ${ }^{4}$ bildet, wie Herbst sagen würde. Er ist damit nicht nur im Sinn einer einfachen numerischen ZeichenManipulation $\mathrm{zu}$ verstehen, wie sie schon in jedem Textverarbeitungspro- 
gramm zur Verfügung steht, sondern genauso als selbstreferenzielle Operation an der eigenen Identität. ${ }^{5}$

Rückläufige Kausalität wirkt sich nun nicht nur innerdiegetisch auf die beschriebene Weise aus, sondern wiederum durch die Hereinnahme der Nullstelle des Diskurses in den Text, jedoch anders als bei Gustafsson durch das Einfließen »der zeitlichen objektiven Geschehen im Leben des Autors, die $\mathrm{zu}$ einem miterzählten Gegenstand des Romanes werden «, ${ }^{6}$ in die Fiktion und nicht durch eine nachträgliche Mise-en-abyme-Struktur. Darauf deutet im Sinn einer Synekdoche bereits die Teilidentität der Namen des Protagonisten Alban Herbst sowie des textexternen, empirischen Autors Alban Nikolai Herbst $(\mathrm{ANH})^{7}$ hin, der selbst wiederum ein Pseudonym der bürgerlichen Person Alexander Michael von Ribbentrop ist. Dieser Austausch scheint in der Tat fast simultan sich zu ereignen und wäre durch selbstreferenzielle, in den neuronalen Netzen seines kognitiven Systems rückgekoppelte Operationen des textexternen Autors ipse in der Auseinandersetzung mit dem gerade entstehenden Text erklärbar - eben als Prozess der Genese von Sinn resp. Bedeutung, der sich in den mannigfachen Austauschbeziehungen zwischen Herbst und Deters auf der innerdiegetischen Ebene widerspiegelt. Hält man sich des Weiteren an das von Herbst (ANH) eingeführte Paradigma der »Regelspirale[...] «, ${ }^{8}$ so wird die Input-Output-Relation rekursiver Rückkopplung hier folglich textintern mimetisch adaptiert und nicht im strengen Sinn von Korrektur/Kontrolle eines identischen, menschlichen Verhaltens oder einer präzise definierten Aufgabe maschinellen Lernens, die nur im Rahmen strenger, objektiver Vorgaben realisierbar wären.

So versteht es sich, dass anstelle des linearen Erzählens zyklische Bewegungen der Narration treten, die nie an den Anfang zurückführen, deren scheinbare Anfänge und Enden also nie identisch, sondern nurmehr ähn-

So scheidet die Interpretation des Geschehens als Teleportation aus, wo - wie in Derek Parfits Gedankenexperiment - eine Person mithilfe eines Teletransporters unter Wahrung der personalen Identität faktisch von der Erde auf den Mars kopiert werden kann, weil es sich in dem beschriebenen, erweiterten Sinn um einen kognitiven Prozess handelt, der auf Inferenz (intendierte Inferenz) beruht. Vgl. Derek Parfit: Reasons and Persons. Oxford: University Press 1986, S. 200 f.

6 A.N. Herbst: Kybernetischer Realismus, S. 90.

7 Wird das Kürzel ANH verwendet, so steht es für den empirischen Autor Alban Nikolai Herbst.

8 A.N. Herbst: Kybernetischer Realismus, S. 81. 
lich sind. ${ }^{9}$ Wenn Herbst (ANH) sagt, »Identität ist geschlossen, Ähnlichkeit hingegen offen ${ }^{10}{ }^{10}$ so ist genau dies der Grund dafür, weswegen sich im Kybernetischen Realismus nicht das Identische einer einmal so erzählten oder zu erzählenden Geschichte reproduziert. Vielmehr ist das, was dem polyperspektivischen Romangeschehen zugrunde liegt, es vorantreibt, »Struktur selber - und damit Prozeß, nicht etwa steuernder Wille [des Autors, d.V.]«. ${ }^{11}$ Es muss allerdings anschlussfähig sein im Prozess des Erzählens und, weil das Emergente nicht zum Produkt gerinnt, ${ }^{12}$ das neu Hinzukommende das bereits Hervorgebrachte im Sinne immer wieder neuer Transformation sowohl verändert reproduzieren als auch transitorisch erweitern. Wenn, wie Herbst schreibt, "auf die Einheitlichkeit der Perspektive zugunsten des Prozesses verzichtet werden [muß] «, kommt es deswegen auf der Seite des Rezipienten ebenso zu »widersprechenden Leseerfahrungen ${ }^{13}$, wie auf der Seite des Schreibenden zu einer Art polymorpher Auflösung homogener Identität, bei der auch eine Vernachlässigung von Gegensatz/Widerspruch wie im psychoanalytischen Primärprozess stattfindet. ${ }^{14}$ Lesenden, denen die Synthese der erzählten Wirklichkeit unter diesen strukturellen Bedingungen nicht gelingt, bricht »die Einheitlichkeit der Romanerfahrung grundsätzlich auseinander. $\aleph^{15} \mathrm{Um}$ dies zu verhindern, muss der Leser seine rein konsumierende Haltung aufgeben, sich gewissermaßen in den Text hineinbegeben. Dies geschieht über die imaginative Besetzung von Leerstellen wie etwa dem eingestreuten Programmkode, der zugleich Ausdruck für einen technischen Systemfehler und ein bestimmtes Erleben des erzählenden Subjekts ist. So ist es zu verstehen, wenn Herbst darauf insistiert, dass er den Leser als Angesprochenen ebenso wie sich selbst als Ansprechenden im Sinne einer Rolle

9 Vgl. ebd., S. 85.

10 Ebd.

11 Ebd., S. 111. Vgl. dazu auch folgende Textstelle zum Verhältnis von Künstler und Werk: »[E]s ist dann tatsächlich nicht mehr von ihm (geschaffen), sondern von etwas durch ihn, etwas, das durch ihn hindurch wirkte. Wobei Sie bitte nicht annehmen wollen, ich spräche von jemandes anderen Willen; es sind vielmehr Strukturen, die hier wirken, Muster, ähnlich den Figurationen immer derselben Grundmodelle von Formung in der Natur.«Ebd., S. 25.

12 Vgl. W. Iser: Emergenz, S. 78.

13 A.N. Herbst: Kybernetischer Realismus, S. 88.

14 Ebenso wäre auch die Vertauschung bzw. das Zusammenfallen der Identitäten von Herbst und Deters auf der innerdiegetischen Ebene dafür ein Beispiel.

15 A.N. Herbst: Kybernetischer Realismus, S. 88. 
in seine Bücher hereingenommen habe. ${ }^{16}$ Auf diese Weise wäre es in der Tat denkbar, den gesamten Prozess literarischer Produktion und Rezeption über Regelkreise vermittelt und ohne Referenzbeziehung im klassischen Sinn zu beschreiben. ${ }^{17}$ Referenz wäre damit nicht als externes (Umwelt-)Ereignis unterstellt, sondern im Sinn eines systemeigenen Zustands etabliert. ${ }^{18}$

Komplexitätstheoretisch deutet dies indessen auf eine Zunahme der Komponenten literarischen Erzählens hin, die $\mathrm{zu}$ berücksichtigen sind. Wenn nicht nur die Strukturen des literarischen Textes und das in ihm Dargestellte, sondern auch das objektive Geschehen im Leben des Autors während des Schreibprozesses zu einem Gegenstand des Romans wird, und zudem, weil es zum zeitgenössischen Geschehen dazugehört, auch die objektive Geschichtszeit des Lesers, ${ }^{19}$ so kommen als fünfte Komponente die selbstreferenziellen Operationen im kognitiven System des Autors hinzu. Diese Komponenten zusammen setzen den Text mit der textexternen Wirklichkeit in ein Verhältnis, bei der die eigentliche Romanhandlung als letzter rein textinterner Bezugspunkt fungiert. Bei Herbst (ANH) sind diese Komponenten Teil einer Matrix, ${ }^{20}$ wie sie auch aus anderen komplexen Systemen, wie etwa der Proliferation von Tierpopulationen in der Populationsgenetik, bekannt ist. Matrices bestehen hier wie dort aus mehrwertig gebundenen Komponenten, die sich in bestimmter Weise kombinieren lassen und so statistische Aussagen über den zukünftigen Verlauf eines Systems erlauben. Herbst nun adaptiert dieses Verfahren für das literarische Erzählen (Schreibprozess), das ihm in ähnlicher Weise auf Wirklichkeit rekurriert wie die biometrische Statistik, indem nämlich durch deren Operationen sich bestimmen lässt, »was wird «. ${ }^{21}$ In der Tat scheint es auch aus komplexitätstheoretischer Sicht plausibel, das bisher global als Prozess qualifizierte Geschehen, das mit der Autopoiese der komplexen literarischen Erzählung (als emergenter Realität) verbunden ist, in dieser Weise, nämlich über die Interaktion von Komponenten innerhalb einer Matrix, $\mathrm{zu}$ operationalisieren.

\footnotetext{
16 Vgl. ebd.

17 Vgl. dazu W. Iser: Emergenz, S. 79.

18 Vgl. Günter Küppers/Wolfgang Krohn: »Selbstorganisation. Zum Stand einer Theorie in den Wissenschaften«, in: Dies. (Hg.), Emergenz (1992), S. 22.

20 Vgl. ebd., S. 98.

21 Ebd.
} 
So ist es auch genau diese Interaktion von Komponenten, die Iser in seinem Essay über Emergenz als »Mehrstelligkeit «22 bezeichnet. Er macht dazu sogar genaue Angaben, wie man sich die Interaktion vorzustellen habe. So ist davon die Rede, dass das Spiel dieser Mehrstelligkeit nach einer aleatorischen Regel verläuft, je nachdem, welche der Komponenten dominant und welche untergeordnet sei. ${ }^{23}$ Nicht nur, dass die Komponenten oder Faktoren innerhalb eines mehrstelligen Systems gegeneinander spielen, sondern auch dass die Modellierung der Faktoren untereinander dem Zufall unterliegt, bedingt, dass derart etwas Unvorhergesehenes, etwas, das es zuvor nicht gab, in die Welt kommen kann. Auch bei Iser sind, ähnlich wie bei Herbst, die Faktoren textinterne wie textexterne. Er nennt »Erzähler, Charaktere, Fabel sowie de[n] intendierte[n] Leser ${ }^{24}$ textintern und versteht Einflussgrößen aus der kulturellen und sozialen Umwelt als textextern. ${ }^{25}$ In der Rezeption durch den Leser treten diese Faktoren in Wechselbeziehung zueinander, woraus sich ein übergreifender Horizont bildet. »Dieser kann«, schreibt Iser, »die Bedeutung des Textes, die vermittelte Erfahrung, die Form der Kommunikation, die inszenierte Vergangenheit, die Sozialkritik und vieles mehr sein. ${ }^{26}$ Ein solcher »Endhorizont [...] « ${ }^{27}$ ist indessen nicht mehr auf die Komponenten rückführbar, wirkt aber im Sinne rückläufiger Kausalität auf diese zurück, indem die Bedingungen des Hervorbringens nachträglich durch das Hervorgebrachte verändert werden. ${ }^{28}$ Wie das zu verstehen ist, dürfte nicht nur am Beispiel der Regelkreise bei Herbst (ANH), sondern auch der alternativen Chronologien in Gustafssons autofiktionalem Roman deutlich geworden sein. Auf diese Weise entsteht auch bei Iser eine »Vernetzung von Komponenten ${ }^{29}{ }^{29}$ deren Zusammenspiel erst das hervorbringt, was zuvor als für sich stehende und nur bedingt miteinander vermittelbare, ontologisch verschiedene Bereiche des literarischen Textes (Diskurs), der textexternen sozialen und kulturellen Realität (Diskursumwelt) sowie dessen, was der literarische Text zur Darstellung bringt (mimetischer Gegenstand), galt. ${ }^{30}$ Diese von Iser stam-

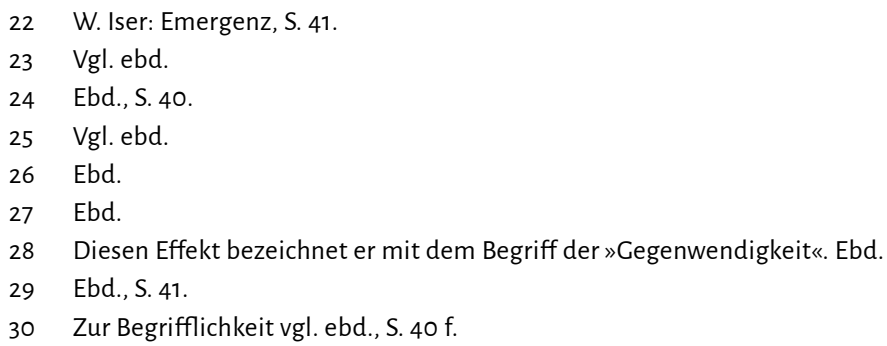


mende Modellierung der Vernetzung mit den dazugehörigen Begriffen wäre komplexitätstheoretisch nun zu spezifizieren.

Denkt man sich, ausgehend vom textexternen Autor, die Interaktion der Komponenten einmal vertikal verkettet, so ergibt sich in Richtung des erzählten Geschehens der Romanhandlung (Diegese) ein iterativer Prozess der Wiederholung auf verschiedenen Ebenen des Systems. Es kommt zu einer Transgression der Grenze zwischen literarischem Text und der textexternen Identität des Autors, in deren Verlauf dieser sich, wie beschrieben, innerhalb der Matrix des Systems "als eine sich mit allen übrigen Figuren permanent wandelnde Figur ${ }^{31}$ in seinen eigenen Text einschreibt. Diese Transgression wiederholt sich iterativ in der Konstellation Alban Herbst (ErzählerProgrammierer) und Hans Erich Deters (erzählte resp. programmierte Figur) zwischen den intradiegetischen Ebenen W3 und W1. Damit wäre das Subjekt aus komplexitätstheoretischer Sicht ein fraktales, das sich auf mehreren, vertikal ineinander verschachtelten Ebenen (Dimensionen) wiederfindet, wiedererkennt, sofern es, wie im Fall ANH - Alban Herbst - Hans Deters, gebrochen in verschiedenen, horizontalen Strukturebenen (Levels von textexterner Realität und intradiegetischen, virtuellen Welten) durch Selbstähnlichkeit aufeinander bezogen bleibt.

Auf der anderen Seite zeigt sich mehr und mehr, dass komplexes Erzählen Hinweise für die Lektüre braucht, wie die Texte gewinnbringend zu lesen seien. Nicht nur, dass der Leser bei der Konstitution des Textes eine eminente Rolle spielt, indem er vor dem Hintergrund seiner subjektiven Wirklichkeit (in analoger Weise wie der Autor beim Schreiben) die Textbedeutung(en) im Lektüreprozess konstruiert. Dabei kann der Text im besten Fall als Instrument innovativer Verhaltenskoordinierung fungieren, mindestens aber im Sinn eines Kognitionsanlasses, bei dem in nicht voraussagbarer Weise Texte rezipiert werden. Der Leser wird zudem, siehe Herbst (ANH), auch durch den empirischen Autor in den Roman mit hineingenommen, wo er im Sinne einer Komponente der als Matrix gedachten, autopoietischen Genese der literarischen Erzählung (als emergentes Geschehen) mit anderen Komponenten, die im Text aufscheinen, interagiert. ${ }^{32}$ Es geht dabei des Weiteren für den Leser als Rolle innerhalb literarischer Kommunikation nicht darum, die >richtige Textbedeutung zu ermitteln, nicht um semantische Objektivität. Angestrebt

31 A.N. Herbst: Kybernetischer Realismus, S. 117.

32 Vgl. ebd., S. $91 \mathrm{f}$. 
wird vielmehr, wie Schmidt schreibt, "auf der Ebene der Kommunikatbildungsprozesse [...] zu einem befriedigenden, als sinnvoll, kohärent und angemessen empfundenen kognitiven Resultat zu kommen $"{ }^{33}$ Dabei kommt es im kognitiven System des Rezipienten, analog zu dem des Autors, zu einer Verkettung selbstreferenzieller Zirkel, die Verstehen als emergentes Phänomen hervorbringen, sodass die Zahl der Komponenten innerhalb des mehrstelligen Systems komplexen literarischen Erzählens noch um eine auf sechs erhöht werden müsste.

Geht man jedoch davon aus, dass Textverstehen nur dann gegeben ist, wenn »die Aktanten ihre Sinnerwartungen erfüllt sehen «, ${ }^{34}$ wie weiter argumentiert wird, so lässt sich auf diese Weise kaum verständlich machen, wie eine Erweiterung des Horizonts, bis hin zur Möglichkeit einer neuen, lebensweltlichen Orientierung des Lesers, durch komplexe Erzähltexte bewerkstelligt werden soll. Diese Möglichkeit ließe sich nur dann sinnvoll in Anschlag bringen, wenn, zugleich mit der durch komplexitätsbedingte Überforderung ausgelösten Perturbation (etwa durch den Clash der Kohärenzen, wie Linke ihn beschreibt), die Faszination durch den im literarischen Text aufscheinenden Horizont beim Leser ausreicht, um sein kognitives System in Richtung auf ein innovatives Verstehen und möglicherweise Verhalten zu triggern. Dass damit eine Krise bisherigen Sinnverstehens und somit eine Dezentrierung des Subjekts verbunden sein kann, ja vielleicht sogar muss, erweist sich jetzt als im Grunde unumgänglich. Dass andersherum der literarische Text allein einen solchen Prozess auszulösen in der Lage wäre, ist andererseits doch kaum denkbar. Dazu bedarf es bestimmter Dispositionen

33 Siegfried ]. Schmidt: »Über die Rolle von Selbstorganisation und Sprachverstehen«, in: Krohn/Küppers (Hg.), Emergenz (1992), S. 293-333, hier S. 309. Der Prozess der Kommunikatbildung, anders gesagt von Verstehen, wird hier mit Gebhard Rusch folgendermaßen als »Qualität des Erlebens eines Vorgangs oder Sachverhalts konzipiert. Wird dieses Erleben als eindeutig, mit bisherigen Erfahrungen übereinstimmend, in sich stimmig und sicher erfahren, so daß sich ein gutes Cefühl von der Angemessenheit und Qualität des Erlebens einstellt und folgen auf dieses Gefühl die erwarteten Verhaltenskonsequenzen, dann liegt Verstehen vor ... Und in diesem Sinn ist Verstehen eine Leistung, die der einzelne Organismus autonom auf der Crundlage seines sinn- und bedeutungsstiftenden (Bezugs-)Systems kognitiver Strukturen erbringt. « Ebd., Fußnote 23. Kommunikatbildung versteht sich somit als selbstorganisierender Prozess, resultierend »aus der selbstreferentiellen Verkettung kognitiver Einzeloperationen, die intellektuelle, affektive und assoziative Komponenten integrieren.«Ebd., S. $318 \mathrm{f}$. 
des Subjekts, die durch Innovationsschübe technischer Natur, negative psychosoziale Einflüsse oder ökonomische wie politische Zwänge zur Migration aus vertrauten Lebenszusammenhängen u.Ä. getriggert werden. Insgesamt Faktoren, die zudem oft in komplexer und damit unvorhergesehener Weise miteinander wechselwirken. Halten wir jedoch fest, dass, sobald das Subjekt, wie im Fall der kybernetischen Poetik, als fraktales vorliegt, dies bereits als Ausdruck weitgehender Dezentrierung betrachtet werden kann. Einmal an diesem Punkt angelangt, ließe sich vielleicht eher davon sprechen, dass das Subjekt sich beständig auf der Spur ist bzw. bleibt, dass es in Formen zeitlicher Zerdehnung vorliegt, wie sie auch für die literarische Kommunikation typisch sind und sich folglich in einem essenzialistischen Sinn aufgrund der radikalen Verzeitlichung von Identität seit der ausgehenden Moderne niemals 'wirklich hat. Wie im Roman Buenos Aires. Anderswelt sucht es nach Zeichen und Spuren eigener Aktivität wie Identität auf den verschiedenen, raumzeitlich parallelen Ebenen simulierter Realität. Der universale Zusammenhang, dass alles mit allem zusammenhänge, von dem eingangs bereits die Rede war, läuft folglich über das Subjekt bzw., im Sinn einer Schaltstelle, durch es hindurch. ${ }^{35}$

Geht man des Weiteren mit Schmidt davon aus, dass das Verstehen literarischer Texte, ja Sprachverstehen generell, nicht einfach so geschieht und weder als Zustand noch als Struktur hinreichend beschreibbar ist, so fragt sich, was aufseiten des Lesers, neben dem Erlebnis der Angemessenheit und Kohärenz, den Perturbationen entgegengesetzt werden kann, welche die Rezeption komplexer Texte erschwerend begleiten. Die empirische Verstehensforschung antwortet darauf, dass Verstehen als zielgerichteter, aktiver Vorgang aufgefasst werden kann, in dessen Verlauf »einem Text ein mentales Modell zugeordnet und bewertet [wird] «, ${ }^{36}$ wie auch in den bisherigen Textanalysen geschehen. Textverstehen lässt sich so als ganzheitlicher, mentaler Konstruktionsakt modellieren, wobei die Modellkonstruktion durch Interaktion zwischen Textinformation und Vorwissen des Lesers erfolgt. ${ }^{37}$ Ein sol-

35 Er kann rein objektiv nicht bestehen. So kann man zwar annehmen, dass auf dem Niveau reiner Gegenständlichkeit eine universelle Verkettung von Ursache-WirkungsBeziehungen existiert, jedoch können die sich nicht selbst erkennen, geschweigen denn verstehen. Dazu bedarf es eines wahrnehmenden Bewusstseins, mithin sich selbst bewusster Subjektivität. S.]. Schmidt: »Selbstorganisation und Sprachverstehen«, in: Krohn/Küppers (Hg.), Emergenz (1992), S. 294.

37 Vgl. ebd. 
ches holistisches Verständnis von Textverstehen wendet sich gegen die hermeneutische Vorstellung von Verstehen als »Bedeutungsentnahme aus einem Text «. ${ }^{38}$ Demnach determiniert der bedeutungstragende Text schon den Verstehensvorgang. Dem steht die Vorstellung einer Interaktion von Sprecherwissen und Textinformation des empirischen Ansatzes entgegen, die Verstehen als subjektzentrierten, strategie- und effizienzorientierten Prozess beschreibt. ${ }^{39}$ Die semantische Dimensionierung von Texten ist folglich sine qua non an die kognitiven Operationen des jeweilig Verstehenden gebunden. Fragt man nach der teleologischen Dimension dieses Prozesses, so lässt sich diese im Anschluss an das bisher Gesagte als intentionaler Vorgang begreifen, »der darauf abzielt, die den Rezipienten umgebende Welt durchsichtig, intelligibel zu machen ${ }^{40}{ }^{\circ}$ Die Zielorientierung besteht, mit anderen Worten, in der Verbesserung der Adaptation des eigenen Verhaltens an die Verhältnisse der komplexer gewordenen Lebenswelt.

Die Frage nach dem, was Dichtung in der gegenwärtigen, durch und durch mediatisierten, komplexen Realität noch soll, lässt sich also von hier aus durchaus plausibel beantworten. Es wundert nicht, wenn Herbst (ANH) mit seiner Konzeption von Literatur im Kybernetischen Realismus in die gleiche Kerbe schlägt: »Weltzusammenhänge sind kompliziert, sind hochkomplex - dieser Komplexität hat sich eine Dichtung, die unserer Zeit noch einigermaßen entsprechen will, zu stellen - ihr muß sie einen Ausdruck verleihen, der zugleich sinnlich fühlbar ist. ${ }^{41}$ Wenn bei ihm das literarische Schreiben im Sinne eines selbstorganisierenden Prozesses mehrwertig gebundene Kausalketten in Bewegung setzt bzw. von einer Wechselwirkung zwischen Komponenten innerhalb einer Matrix die Rede ist, ${ }^{42}$ so findet mit Iser beim Leser ein analoger Vorgang statt, der sich kognitionstheoretisch zudem als zielorientiert und in ähnlicher Weise intentional entpuppt, sobald es um das kognitive Verstehen im Text aufscheinender, komplexer Lebenswirklichkeiten geht. Somit kann Literatur durch die beschriebenen, komplexen Prozesse der Rezeption, besonders durch die Fähigkeit zur kreativen Uminterpretation, Subjekte für bisher so nicht wahrgenommene

\footnotetext{
38 Ebd., S. 295.

39 Vgl. ebd.

40 Ebd.

41 A.N. Herbst: Kybernetischer Realismus, S. 112.

42 Vgl. ebd., S. 91.
} 
Zusammenhänge in einer immer komplexer werdenden Lebenswelt sensibilisieren. Und selbst dort, wo Leser wie Autoren in einem objektiven Sinn mit der Erfahrung auswegloser Negativität konfrontiert sind, an der sie als einzelne nichts ändern können, man denke an Solschenizyn, Levi, Beckett oder Camus, beginnt die Literatur an diesen Grenzen zu arbeiten, indem sie darüber spricht. Grenzen, die im Sinn subversiver Lektüre auch durch die Kommunikation der Leser untereinander wenn nicht transzendiert, so doch wenigstens wahrnehmbar gemacht werden können. 



\section{Vom Umgang mit diskursiver Komplexität im Kontext emotionaler und kognitiver Partizipation an der komplexen Erzählung}

Es ist hier nun der Punkt erreicht, sich noch einmal klar zu machen, dass $\mathrm{Zu-}$ nahme von Komplexität, wie sich auch im nächsten Abschnitt am Beispiel der komplexer gewordenen, internen Verfasstheit transkultureller Subjekte mit Welsch zeigen wird, zugleich durch ein Anwachsen der Möglichkeiten, dieses Phänomen wissenschaftlich zu erklären resp. es zu verstehen, beschreibbar ist. Dies gilt nun ebenso für das Phänomen des Verstehens als solchem, das es zweifellos gibt, das man jedoch auf verschiedene Weisen erklären kann. Ob nun kognitionstheoretisch, konnektionistisch oder hermeneutisch, ${ }^{1}$ es zeigt sich an dieser Stelle aufs Neue, dass der Zunahme von Komplexität aufseiten des Phänomens ein Anwachsen der Methoden/Diskurse zu seiner Bewältigung korrespondiert. ${ }^{2}$ Analog verhält es sich etwa auch mit den Perso-

Es können hier aus Cründen der Beschränkung längst nicht alle methodischen Ansätze zur Erklärung von Verstehen erwähnt werden. Selbst innerhalb des kognitiven Ansatzes gibt es noch eine Vielzahl von Varianten, die Verstehen u.a. als Errechnen von Eigenwerten kognitiver Systeme ( $H$. v. Foerster), Konstruktion viabler Kognitionen ( $E$. v. Clasersfeld), rekursives Selbstverstehen (K. Krippendorff), Kommunikatkonstruktion (S.]. Schmidt) sowie Fremd- und Selbstattribution (G. Rusch) modellieren. In der Lingusitik und Sprachpsychologie wird Verstehen weitgehend als Informations- bzw. Textverarbeitung konzipiert. Dabei wird auf der Basis von frames und scripts als mentalen Modellen von der subjektiv konstruierten, mentalen Repräsentation wahrgenommener Cegenstände ausgegangen. Vgl. dazu Cebhard Rusch: »Verstehen«, in: Ansgar Nünning (Hg.), Metzler Lexikon Literatur- und Kulturtheorie. Ansätze - Personen Grundbegriffe, 2. überarb. u. erweit. Aufl., Stuttgart et al.: ].B. Metzler 2001, S. 658. interagieren, zeigt sich auch an Sandra Richters Weltgeschichte der deutschsprachigen Literatur (2017). Neben dem Clobalisierungsdiskurs überlagern sich hier in vielfältiger 
nalpronomen, deren Bedeutung und Funktion je Diskurs verschieden sind. Das Ich oder das Er/Sie, die im literarischen Text die Stelle des Subjekts vertreten, sind weder identisch mit denen der Neurowissenschaften noch der Philosophie des Subjekts und ebenso wenig der Gesellschaftswissenschaften. Über die Personalpronomina kreuzen sich somit auf dem Feld der literaturwissenschaftlich-narratologischen Analyse, zu der die vorliegende Studie gehört, die genannten Diskurse. Die Pronomen sind als Bedeutungsträger in den verschiedenen theoretischen Kontexten bzw. Frames je unterschiedlich kodiert, bedeuten also je etwas anderes, mit u.U. auch identischen Teilbedeutungen (Konnotationen). ${ }^{3}$ Etwas Gleiches bedeutet folglich in verschiedenen Wissenszusammenhängen etwas anderes, was wiederum die klassische Definition für Mehrfachkodierung ist, wie sie analog von Nassehi im Zusammenhang der Definition von Komplexität verwendet wird, nämlich als Zustand (Bedeutung), der zugleich mehrere weitere Zustände (Bedeutungen) annehmen und nicht kausal aus der Vergangenheit hergeleitet werden kann. Es geht damit um diese Schnittstellen zwischen den Diskursen, die man in der Absicht, verschiedene Theorien aneinander an-oder miteinander kurzzuschließen, vielleicht ebenso gut als Schaltstellen bezeichnen könnte, um auf diese Weise das zu erzeugen, was man als Resonanz bezeichnet, nämlich das Mitschwingen verschiedener Kontexte bei der Bestimmung eines umfassenderen Phänomens, hier des komplexen Erzählens. ${ }^{4}$

Vor dem Hintergrund einer solchen Dispersion und der damit verbundenen, sich stetig fortsetzenden Ausdifferenzierung des Wissens stellt sich freilich auch die Frage, ob Wissen unter diesen Bedingungen überhaupt noch allgemein gültig sein kann oder nicht mehr und mehr partikular wird. Findet sich Wissen dann nur noch in Form situativer Einbettung als Embedded knowledge (kontextgebundenes Wissen)? So ließe sich beispielsweise die Frage, was versteht man eigentlich genau unter der Bedeutung der Personal-

Weise nationale und fremdländische Literaturgeschichten, Transkulturalismus, Übersetzungstheorie und Kulturgeschichte.

In ähnlichem Zusammenhang spricht Koschorke auch von Doppelkonditionierungen, die aus der Überlagerung zweier bzw. mehrerer Zeichenregimes resultieren. Da die Zeichenregimes wechselseitig unassimilierbar bleiben, weil ein Übersetzungsschlüssel zwischen den aufeinandertreffenden Ordnungsprinzipien fehlt, kommt es zu epistemischen Konflikten, die perennieren, weil sie sich nicht auflösen lassen. Vgl. Albrecht Koschorke: Wahrheit und Erfindung. Crundzüge einer Allgemeinen Erzähltheorie, 2. Aufl., Frankfurt a.M.: S. Fischer 2012, S. 368 f.

4 Zur Resonanz zwischen Diskursen vgl. D. Wrobel: Postmodernes Chaos, S. 18. 
pronomen der 1. und 3. Person Singular, so allgemein formuliert dann kaum mehr sinnvoll stellen. Man muss sich nun zur Beantwortung der Frage schon selbst in die betreffende Situation begeben, die je Wissenszusammenhang sehr unterschiedlich ausfallen kann, um die damit verbundenen Erfahrungen (im Sinne von Wittgensteins Sprachspielen) überhaupt machen zu können. Dieser Hinwendung zu Situated learning (situationsgebundenes Lernen) bzw. Embedded knowledge, die mit einer Relativierung, gar Abwertung dessen einher geht, was in der Kognitions- und Lernpsychologie als Inert knowledge (träges Wissen $)^{5}$ bezeichnet wird, entspricht gerade in der neueren Rezeptionsforschung einem zunehmenden Interesse an der performativen Beteiligung des Lesers am Text durch emotionale Partizipation. Dieser Ansatz ist verbunden mit einer Abwendung von traditionellen Formen der Literaturrezeption, genannt seien hier literaturwissenschaftliche Hermeneutik, Biografismus und psychoanalytische Lesarten. Es geht dabei vor allem darum, dass der Leser jenseits des intelligiblen Verstehens von Sinn und Bedeutung vor allem über die emotionale Partizipation am Text aktiviert wird. Es kommt also weniger auf die Übertragung bzw. Konstruktion von Information/Wissen an, sondern in erster Linie auf das (Mit-)Erleben, der im Text dargestellten Inhalte. Wie Teresa Hiergeist gezeigt hat, geschieht dies vor allem über »textspezifische Beteiligungsstruktur[en] «, ${ }^{6}$ die im Sinn einer Mithandlungsoption als Atmosphäre, Gefühl oder Motivation vom Leser in Interaktion mit dem Text generiert werden. Dies bedeutet mit anderen Worten, dass literarische Texte nicht länger autonom existieren, sondern sich zu einem großen Teil im Rahmen der Lektüre als interaktiver und performativer Prozess konstituieren. ${ }^{7}$

Dabei fungieren sowohl die Idee der Offenheit des Zeichens (Derridas Differance, Ecos unendliche Semiose) als auch die Vorstellung der Lektüre als Form der Écriture (Barthes), bei der die selektive Wahrnehmung des Lesers als kreativer Akt der Auswahl verstanden wird, als Voraussetzungen für ein Verständnis des Lesens als produktiver Tätigkeit. Somit ist von einer grundlegenden Pluralität des literarischen Textes auszugehen. ${ }^{8}$ Bedeutung gibt es

Zum Zusammenhang von kontextgebundenem Wissen, situationsgebundenem Lernen und trägem Wissen vgl. https://de.wikipedia.org/wiki/Tr\%C3\%A4ges_Wissen vom 16. 02.2021 .

6 Teresa Hiergeist: Erlesene Erlebnisse. Formen der Partizipation an narrativen Texten, Bielefeld: transcript 2014, S. 13.

7 Vgl. ebd.

8 Vgl. ebd., S. 28 
folglich nur noch in der Mehrzahl und nicht mehr in einem absoluten Sinn. Dies wiederum hat zur Folge, dass die »Diversifikation der Interpretationsmöglichkeiten « ${ }^{9}$ zur Bedingung sine qua non der Kommunikation über literarische Texte wird und aus diesem Grund die Vorstellung vom Roman als abgeschlossenes Bedeutungskonstrukt mit transzendentalem Sinn endgültig der literaturexegetischen Vergangenheit angehört. Komplexitätstheoretisch betrachtet, kommt es nun durch die Einsicht in die Abhängigkeit der Literaturinterpretation von der Conditio des Lesers, die sowohl historisch als auch gesellschaftlich und individuell-situativ zu denken ist, zu einer Erhöhung des Komplexitätsgrades. Dies ist umso naheliegender, als das komplexitätsreduzierende Ursache-Wirkungs-Prinzip zwischen Text und Leser mehr und mehr der komplexeren Vorstellung einer rekursiven Interaktion zwischen beiden weicht. $^{10}$

Damit wird in der Tat die Position der empirischen Literaturwissenschaft, deren Erkenntnisinteresse sich bereits in Richtung Aneignungsvorgang des Lesers verschoben hatte, in wesentlichen Aspekten erweitert und vertieft. Wenn nunmehr der performative Erlebnischarakter der Rezeption im Vordergrund steht, werden damit sowohl die Idee der Kommunikation einer Bedeutung zwischen Autor und Leser als auch die Vorstellung eines Wirkungstransfers vom Werk auf den Rezipienten (Reiz-Reaktions-Schema) als exklusive Muster der Erklärung rezeptiver Prozesse weitgehend obsolet. Begründet wird dies mit der Kritik, dass im ersten Fall der Sender (Autor) in der Übermittlungssituation real abwesend sei und im letzteren die Textinformation die Reaktion des Lesers zu sehr determinieren würde. ${ }^{11}$ Solange der Lektürevorgang im Wesentlichen Sinn- und Bedeutungsfindung bleibt (wie vorzugsweise in der Hermeneutik), kommt es demnach noch nicht zur emotionalen Partizipation des Lesers im Sinn von Hiergeist. Dazu wäre die Erfassung der Präsenzdimension literarischer Texte und somit die Verlagerung der Beteiligung des Lesers in den Roman hinein erforderlich. Anders bliebe, wie Hiergeist weiter ausführt, der Erlebnischarakter literarischen Lesens unberücksichtigt. ${ }^{12}$

\footnotetext{
9 Ebd.

10 Vgl. ebd., S. 29.

11 Vgl. dazu ebd., S. 33-41.

12 Vgl. ebd., S. $32 \mathrm{f}$.
} 
Insgesamt lässt sich somit eine Tendenz weg vom »Bedeutungszentrismus ${ }^{13}$ hin zu einer interaktiven Präsenzerfahrung (Gumbrecht) mit literarischen Texten konstatieren. ${ }^{14}$ Dies bedeutet jedoch nicht, dass die kognitive Partizipation völlig hinter der affektiven Involvierung des Rezipienten zurücktritt. Für ein umfassendes, interaktives Zusammenspiel von Text und Leser sind neben der Emotion nach wie vor kognitive Aspekte (Verstehen von Sinn und Bedeutung bzw. deren Konstruktion durch Aufnahme und Verdichtung informationeller Daten des Textes) als Modus der Beteiligung am Text unverzichtbar, denn erst durch sie entsteht beim Leser eine mentale Repräsentation der erzählten Welt. Dies geschieht, indem leserseitig sprachliches, kulturelles, literarisches und situationelles Wissen mit dem Textangebot rekombiniert und auf diese Weise das Erlebnis einer sinneren Welt < produziert werden kann. Als weitere Modi der Beteiligung, die einander nicht ausschließen, sondern sich gegenseitig bedingen, kommen die evaluative Partizipation, in deren Verlauf der Leser die im Text dargestellten Figuren und $\mathrm{Zu}$ sammenhänge bewertet und diese mit seinem eigenen, inneren Normsystem abgleicht und schließlich die ästhetische Partizipation hinzu, bei der die Einheit der vorhergehenden Partizipationsmomente in Form einer Synthese als Meta-Erlebnis etabliert wird.

Es geht folglich darum, ein neues Bewusstsein für die Bandbreite der Beteiligungsmöglichkeiten am Text zu schaffen. Wenn der literarische Text gewissermaßen erst durch das Mithandeln des Lesers auf emotionaler, kognitiver, evaluativer und ästhetischer Ebene entsteht, bekommt nicht nur das Lesen dadurch Handlungscharakter im performativen Sinn, sondern es kommt rezeptionstheoretisch aufgrund der Zunahme der beteiligten Fähigkeiten zu einer Erhöhung der Komplexität des Gesamtprozesses. Wenn der Rezipient des Weiteren durch den »Erlebniswert der Sprache «, ${ }^{15}$ über die ikonische Vergegenwärtigung des Gelesenen und die Ausbildung kognitiver Partizipationsmuster in eine ähnliche, emotional-affektive und kognitiv-epistemische Disposition versetzt werden kann wie der Erzähler bzw. die Figur im literarischen Text, ist dies wiederum die Voraussetzung dafür, sich evaluativ zu dessen Gefühlen, Meinungen, Urteilen und Sichtweisen zu verhalten. ${ }^{16}$ Dabei ist die

\footnotetext{
13 Ebd., S. 41.

14 Vgl. ebd., S. 41-44.

15 Ebd., S. 371.

16 Vgl. ebd.
} 
Qualität der emotionalen Beteiligung ausschlaggebend dafür, wie die Bewertung des Protagonisten ausfällt, wird also dann positiv ausfallen, wenn die affektive Partizipation positiv besetzt ist. Je nach Intensität und Qualität des erlebten Gefühls kommt es zu einer Annäherung oder zu einer Distanzierung im Fall der negativen Bewertung bzw. der völligen Unzugänglichkeit des Romangeschehens. Das Verhältnis von Nähe und Distanz zwischen Leser und Figur bewegt sich dabei auf einer Skala mit einer großen Vielfalt an Möglichkeiten, die vom völligen Eintauchen in die Welt der Erzählung (Immersion) bis hin zur Verstörung reicht, wenn es zur Bildung widersprüchlicher Emotionen kommt und das Gesamtgeschehen nicht stimmig synthetisiert werden kann. Bei vollständiger Einfühlung und Identifikation kann die erzeugte Nähe hingegen so unmittelbar sein, dass der Rezipient kaum mehr zwischen Fiktion und Realität zu unterscheiden vermag. Schließlich wird eingeräumt, dass die Partizipation nicht notwendigerweise an eine Figur gekoppelt ist. Denkbar ist ebenso gut die Nähe zur Erzählsituation als solcher, zur Atmosphäre der erzählten Welt (Effet de réel nach Roland Barthes) oder die Übereinstimmung mit den Wertungen der erzählenden Instanz. ${ }^{17}$

Wenn Hiergeist abschließend von »der Unermesslichkeit der Interaktionsangebote « zwischen Leser und Text, »den zahllosen unterschiedlichen Positionen, die der Leser gegenüber narrativen Texten einnehmen kann ${ }^{18}{ }^{18}$ spricht, so ist dies zugleich ein Beleg dafür, dass die Erweiterung der Perspektive über die textimmanente Betrachtungsweise hinaus, zu einem starken Anwachsen der Komplexität, verstanden als Möglichkeit der Kombination divers skalierbarer Positionen, führt. Dabei wird das Prinzip unidirektionaler Wirkung/Verursachung (vom Autor zum Text, vom Text zum Leser) weitgehend abgelöst von der Vorstellung einer prozesshaften Wechselwirkung (Interaktion) zwischen den genannten Instanzen, sei es als Produktionslogik, die sich als kybernetischer Regelkreis versteht (ANH, Gustafsson, Proust), oder im Sinn der performativen Interaktion des Rezipienten mit dem Text (Hiergeist). Wiederum ist es so, dass die systemische Ausgliederung der literarischen Kommunikation als Gesamtphänomen, bestehend aus den Partitionen Autor, Text und Leser, in verschiedene Teildiskurse (Produktionsästhetik, Narratologie, Rezeptionsforschung), zu einer weiteren Ausdifferenzierung der gesonderten Perspektiven/Aspekte des Phänomens erzählender Literatur beigetragen hat. Jeder dieser Zweige ist mittlerweile

17 Vgl. dazu insgesamt ebd., S. 372-377.

18 Ebd., S. 377. 
so umfangreich, dass kaum jemand noch in der Lage wäre, das gesamte Feld der Beiträge zu überschauen.

Zunahme von Komplexität lässt sich folglich nicht festmachen an der Entwicklung in einem isolierten Teilbereich. Sie teilt sich aufgrund interaktiver Kommunikationsprozesse nahezu unmittelbar oder mit geringer zeitlicher Verzögerung in allen möglichen Direktionen mit, wobei aufgrund der Simultaneität gegenwärtiger digitaler Kommunikationsformen der Ursprung/Ausgangspunkt der Entwicklung nicht mehr eindeutig zurückverfolgt werden kann. Aufgrund dieser Lage ist auch davon auszugehen, dass die Zunahme von Komplexität in der Lebenswelt, wie bereits eingangs prognostiziert, sich in der beschriebenen Weise auf die Rezeption literarischer Texte auswirkt, auch wenn diese als solche vielleicht nicht einmal übermäßig komplexe Binnenstrukturen aufweisen. Eine komplexer gewordene Wirklichkeit bietet wohl für nahezu jede literarische Erzählung mehr Möglichkeiten des Anschlusses/der Konnektion an neue, in ihr selbst ursprünglich gar nicht angelegte Kontexte, die aus der Wirklichkeit des Lesers kommen, als noch zuvor. Wenn Hiergeist am Schluss ihrer Ausführungen resümiert, dass die Lektüre den Leser aufgrund der genannten Partizipationsmomente verändern könne und sogar einräumt, dass er danach nicht mehr der gleiche sei wie davor, ${ }^{19}$ so kann man dies durchaus im Sinn des lebensverändernden Potenzials komplexer literarischer Texte nach Mieth verstehen. Lektüre ist demnach nicht nur in der Lage, den Leser emotional zu bewegen und ästhetisch $\mathrm{zu}$ faszinieren, sondern ihn darüber hinaus aus fixiertem Verstehen $\mathrm{zu}$ neuen Möglichkeiten kognitiver Erkenntnis und schließlich, wie schon Sartre wusste, sogar zu gesellschaftlichem Engagement zu motivieren.

Wenn bisher davon gesprochen wurde, dass der Leser die Welt des Textes auf je subjektive Weise mit seiner eigenen Wirklichkeit verknüpft und dass eben daraus, sofern dies in Form eines sich selbst verstärkenden, positiv rückgekoppelten Prozesses geschieht, das entsteht, was man als Emergenz bezeichnet, nämlich etwas - neue Einsichten, Ideen, Handlungsoptionen -, das als solches noch nicht in den Komponenten, aus denen es hervorgegangen ist, enthalten war, so ist fraglich, wie dieser Prozess der Fusion semantischer Einheiten aus dem literarischen Text mit den affektiven sowie kognitiven Gegebenheiten des Lesers sich im Einzelnen gestaltet. In der neueren, kognitionslinguistischen Theorie bezeichnet man diesen Fusionsvorgang 
auch als Blending. Gemeint ist damit die Fähigkeit, mindestens zwei kognitive Repräsentationen/Räume (Spaces) aus verschiedenen konzeptuellen Bereichen als Input im Prozess des Blendings (Fusion, Mischung) so miteinander zu (re-)kombinieren, dass daraus ein Blend mit emergenten Eigenschaften resultiert. ${ }^{20}$ Dabei ist dieser Vorgang nicht nur für den vorliegenden Zusammenhang relevant, also die Frage, wie neue Bedeutung, neuer Sinn im Rahmen der Rezeption literarischer Texte generiert wird, sondern darüber hinaus, wie Mark Turner annimmt, im Grunde in allen Lebensbereichen, besonders aber in den Wissenschaften und Künsten, sobald es darum geht, durch Kreativität neue Lösungen für ein Problem zu finden. ${ }^{21}$

Wie Sophia Wege gezeigt hat, ist unter den vier verschiedenen Typen des Blendings für die Literaturwissenschaft der sog. Double-scope-blend am interessantesten, weil aus ihm ein emergentes Produkt resultiert, das sich "aus widersprüchlichen mentalen Konzepten zusammensetz[t]«. ${ }^{22}$ Auf diese Weise lässt sich auch die Fähigkeit der Literatur zur Fusion verschiedenartigster Realitäten erklären, auf die eingangs bei der Engführung von Komplexität und Literatur zum komplexen Erzählen hingewiesen wurde. Des Weiteren wird vor diesem Hintergrund verständlich, wie die Entstehung fiktiver Identitäten erklärt werden kann. Ja, im Grunde lässt sich so das Fiktive des literarischen Textes, etwas anders als es Iser mit seiner Vorstellung der Grenzüberschreitung getan hat, ${ }^{23}$ generell als Fusion der kontrafaktischen Fiktion mit der faktualen, lebensweltlichen Realität des Lesers bestimmen. ${ }^{24}$ Interessant

20 Vgl. dazu Sophia Wege: »Die kognitive Literaturwissenschaft lässt sich blenden. Anmerkungen zum Emergenz-Begriff der Blending-Theorie«, in: Roman Mikuláš/Sophia Wege (Hg.), Schlüsselkonzepte und Anwendungen der Kognitiven Literaturwissenschaft, Münster: Mentis 2016, S. 243-260, hier S. $243 \mathrm{f}$.

21 Vgl. ebd., S. 247. Mark Turner gilt neben Gilles Fauconnier als Vater des BlendingPrinzips. Vgl. dazu Gilles Fauconnier/Mark Turner: The way we think. Conceptual blending and the minds hidden complexities, New York: Basic Books 2002.

22 S. Wege: »Kognitive Literaturwissenschaft«, in: Mikuláš/Wege (Hg.), Schlüsselkonzepte (2016), S. 245.

23 Vgl. Wolfgang Iser: Das Fiktive und das Imaginäre: Perspektiven literarischer Anthropologie, Frankfurt a.M.: Suhrkamp 1991, S. 18-23.

24 Des Weiteren kann man auf diese Weise, wie Natalia Igl hervorgehoben hat, das Verhältnis von inner- und außertextuellem Raum in literarischen Erzählungen mit narrativer Rahmen-Binnen-Struktur (z. B. Herausgeberfiktionen), wie sie seit der Romantik bekannt sind, gewinnbringend analysieren. Vgl. Natalia Igl: »Romantische RahmenBinnen-Konstruktionen als >Mapping`von inner- und außertextuellen Räumen«, in: Mikuláš/Wege (Hg.), Schlüsselkonzepte (2016), S. 81-100. Beide Bereiche können mit- 
ist nun, dass man in der kognitiven Literaturwissenschaft von einer gleichartigen »kognitiven Prozessierung von Literatur und Welt ${ }^{25}$ ausgeht. Dieser Ansatz geht bereits auf Bernd Scheffer zurück, der auch »von einer Parallelität von Wahrnehmung und Interpretation ${ }^{26}$ gesprochen hat. Gemeint ist damit, dass sowohl der reale als auch der fiktionale Raum auf der Grundlage weitgehend identischer, kognitiver (Wahrnehmungs-)Prozesse in einer »[e]ndlos autobiographischen Tätigkeit der Wahrnehmung « ${ }^{27}$ erzeugt werden und dass dies die Voraussetzung dafür ist, dass überhaupt eine Interaktion zwischen literarischem Text und der Lebenswirklichkeit des Rezipienten zustande kommen kann. In beiden Fällen sind es mentale Repräsentationen, die zur Bildung von Vorstellungsinhalten führen, die im einen Fall jedoch nicht senso-motorisch mit dem aktuellen Wahrnehmungs- und Bewegungsraum des Subjekts rückgekoppelt, sondern im Rahmen der Lektüre imaginativ entfaltet wird. ${ }^{28}$

Komplexitätstheoretisch zeichnet sich, wie schon am Beispiel der sich kreuzenden Diskurse deutlich wurde, wiederum ab, dass es zur Erklärung der Fusion verschiedener Wirklichkeitsbereiche über den/im literarischen Text mehrere Ansätze gibt. Man mag sich fragen, ob damit, über die Pluralität der Ansätze hinaus, ein wesentlicher Erkenntnisgewinn verbunden ist, wenn man für die Verschmelzung von inner- und außertextuellem Raum je nach Kontext nun die Blending-Theorie, den Begriff der Randbedingungen aus der Theorie komplexer Systeme, die n-dimensionalen Skalierungen der fraktalen Geome-

einander kombiniert werden und sich dabei überlagern, etwa wenn in einer Vorrede durch den Rahmenerzähler Erzählraum und erzählter Raum, die eigentlich durch die Rahmen-Binnen-Struktur klar voneinander getrennt sind, metaleptisch in Beziehung gesetzt und sich miteinander aus- bzw. vertauschen können. Beispiele dafür wären E.T.A Hoffmanns Die Serapions-Brüder (1819/21) sowie Wolfgang Hilbigs Erzählung Der Brief (1981).

25 Ebd., S. 85.

26 Bernd Scheffer: Interpretation und Lebensroman. Zu einer konstruktivistischen Literaturtheorie, Frankfurt a.M.: Suhrkamp 1992, S. 7.

27 Ebd., S. 24. Bei Scheffer findet sich literaturwissenschaftlich gewendet auch die aus der neurobiologischen Kognitionsforschung stammende Einsicht, dass Sinn und Bedeutung vom Subjekt des Rezipienten ipse durch selbstreferenzielle Prozesse (Zirkel) erzeugt werden. Er verwendet dafür den Begriff der Selbstbeschreibung: »WirklichkeitsKonstruktionen (und Textbedeutungen) werden in einem Prozeß der Selbstbeschreibung erzeugt und aufrechterhalten. « Ebd.

28 Vgl. dazu auch N. |gl: »Romantische Rahmen-Binnen-Konstruktionen«, in: Mikuláš/ Wege (Hg.), Schlüsselkonzepte (2016), S. 85. 
trie oder eine Komponenten-Matrix heranzieht. ${ }^{29}$ Offenbar nicht in dem Sinn, dass sich daraus ohne weiteres ein Master-Diskurs entwickeln ließe (bzw. als emergente Wirkung hervorginge), der alle genannten Teildiskurse zu integrieren in der Lage wäre. Dies wäre nur dann vorstellbar, wenn man das Design der Komplexitätsforschung adaptieren könnte, wo es ja auch verschiedene, an sich eigenständige Diskurse aus verschiedenen Wissensbereichen gibt, die unter den Hinsichten der Berechenbarkeit, der Wahrscheinlichkeit, der Information, der Systemdynamik u.a. im Master-Diskurs der Komplexitätsforschung subsumiert und dabei entlang der genannten Kriterien gewissermaßen vereinheitlicht werden. Für das Feld literarischer Komplexität käme freilich nur ein Anschluss an den übergeordneten Diskurs ästhetischer Komplexität in Frage, wie ihn Koschorke bereits ins Gespräch gebracht hat.

29 Gleiches gilt für die Seite des Subjekts der Rezeption. Ob endlos autobiografische Tätigkeit der Wahrnehmung (B. Scheffer), unendliche Semiose (U. Eco) oder endloser Aufschub einer finalen Bedeutung/eines finalen Sinns in der Differance (]. Derrida), je nach Paradigma verschiebt sich der Fokus bzw. ändert sich der Kontext, wobei das Phänomen, hier die Unabschließbarkeit von Bedeutungs- und Sinnbildungsprozessen, weitgehend das gleiche bleibt. 


\section{Transkulturelles vs. hermeneutisches Modell des Verstehens unter Bedingungen der Komplexität}

Bedenkt man im gegebenen Zusammenhang das, was Wolfgang Welsch als "interne Transkulturalität der Individuen " ${ }^{1}$ bezeichnet hat, also den Umstand, dass in gegenwärtigen Gesellschaften die Menschen zunehmend »durch mehrere kulturelle Herkünfte und Verbindungen « geprägt sind, so kann das als weitere Erklärung für die Zunahme innerer Pluralität und der damit zusammenhängenden komplexen Relationen, die ein Subjekt herzustellen vermag, verstanden werden. Auch hier kommt es analytisch zu einer Transgression oder, genauer gesagt, $\mathrm{zu}$ einer Durchdringung subjektiver Identität diesmal mit verschiedenen kulturellen Mustern bzw. ethnischen Zugehörigkeiten. Nach Welsch lässt sich eine solche komplexe Verquickung unterschiedlichster Elemente weder auf der gesellschaftlichen Makroebene noch auf der Mikroebene des individuellen Subjekts kaum mehr zeitgemäß auf Basis des älteren interkulturellen Kulturmodells erklären, das mit Herder noch davon ausgeht, dass Kulturen sich rein als intern homogene Monaden gegenüberstehen, ohne dass die Möglichkeit einer Vermischung real bestünde. Dieser "Differenz-Fiktion ${ }^{3}$ setzt Welsch die Vorstellung einer realen kulturellen Durchdringung entgegen, die gerade in der Gegenwart durch Prozesse der Globalisierung eine starke Beschleunigung erfahren hätte. ${ }^{4}$ Demnach leistet diese reale Entwicklung schon das, was der interkulturelle

Wolfgang Welsch: »Was ist eigentlich Transkulturalität?«, in: Dorothee Kimmich/Schamma Schahadat (Hg.), Kulturen in Bewegung. Beiträge zur Theorie und Praxis der Transkulturalität, Bielefeld: transcript 2012, S. 31.

2 Ebd., S. 30.

3 Ebd.

4 Vgl. ebd., S. 35. 
Dialog durch hermeneutische Annäherung und "Horizontverschmelzung « 5 erst ermöglichen soll, nämlich die Möglichkeit des gegenseitigen Verstehens einander zutiefst fremder Kulturen. Dabei verhindere die im interkulturellen Dialog gemachte Voraussetzung der Inkommensurabilität der Kulturen letztlich gerade ein tieferes Verständnis, da die Hermeneutik des Verstehens immer gebunden sei an den Horizont der eigenen Herkunftskultur und diesen, indem sie alles auf ihn zurückführe, nie transzendieren könne. ${ }^{6}$

Der interkulturelle Dialog erweist sich so bereits in seinen theoretischen Implikationen als selbstwidersprüchliches Unterfangen. Die Realität der Globalisierung zeigt hingegen, so ließe sich ergänzen, dass in allen wesentlichen Bereichen von der Ökonomie bis zum Bildungsaustausch, wenn nicht gegenseitiges Verstehen hermeneutischer Tiefenstrukturen, zumindest doch koordiniertes Handeln möglich ist. So gesehen ließe sich der hermeneutische Ansatz des interkulturellen Denkens, wenn er alles im Rahmen der Herkunftskultur verstehbar machen will, sogar als Komplexität reduzierend begreifen. Anders der Transkulturalismus, der auf den fahrenden Zug der globalen Entwicklung aufgesprungen ist und angesichts der zunehmenden Herausforderungen durch Komplexität auf die gegenseitige Kommunikation im Medium der neuen Lingua franca des Englischen setzt, das als am stärksten globalisierte Sprache weit weniger als das Deutsche an ein beschränktes Territorium und damit einen beschränkten Wirkungskreis gebunden ist. Verlässt man das Bezugssystem deutscher Sprache und Kultur, so wird schnell erkennbar, dass der größte Teil erzählender Literatur, die global rezipiert wird, auf Englisch erscheint. Globalisierung in diesem Sinn ist jedoch eine weitgehend andere Kategorie als Interkulturalität, wie sie in der Germanistik verstanden wird, wo etwa Beziehungen zwischen Zentrum und Peripherie, das Denken in Ähnlichkeiten, die Destabilisierung von Identitäten, die Undefinierbarkeit von Alterität, die Verschränkung verschiedener Blickpunkte innerhalb dessen, was sich als Histoire croisée verstehen lässt, eine wichtige Rolle spielen. Globale Verwertbarkeit des Schreibens, das sich nicht mehr für die Heterogenität erlebter Wirklichkeit interessiert, wäre gemessen an der Komplexität dieser Zusammenhänge hingegen gleichbedeutend mit einem Verlust an Individualität, worauf schon Peter Gossens in einem Aufsatz über Neue Weltli- 
teratur hingewiesen hat. ${ }^{7}$ Ein solcher Blick von nirgendwo hat zur Folge, dass Autoren sich nicht mehr an authentischen Erfahrungen mit kulturell verortbaren Bezügen, sondern in erster Linie an der weltweiten Markttauglichkeit ihres Produkts orientieren.

Vorderhand scheinen damit, solange man sich im Bereich des Warenverkehrs, des Handels mit Geschäftsmodellen und Dienstleistungen, der internationalen wirtschaftlichen und politischen Verflechtungen, also der mehr oder weniger verdinglichenden bzw. handlungsgebundenen Kommunikation bewegt, der kombinatorischen Zunahme von Komplexität keine Grenzen gesetzt zu sein. Es fragt sich nun aber, ob dieser Ansatz auch dann noch plausibel bleibt, wenn es sich nicht um gewöhnliche Handelswaren, sondern um kulturelle Güter handelt, die, wie im Fall der erzählenden Literatur in einer nicht-globalisierten Sprache, wie es das Deutsche ist, jenseits der Grenzen des deutschsprachigen Sprach- und Kulturraums mit dem Problem der Nichtrezipierbarkeit konfrontiert sind. Hier kommt der Umstand ins Spiel, dass sprachliche Gemeinschaften durch ihren weitgehend exklusiven, semantischen Kode dem Verstehen durch Gruppen mit fremder Sprache unzugänglich sind. Sie erweisen sich - bildhaft gesprochen - als nach innen gewissermaßen abgeschottet durch den Abgrund ihrer sprachlichen Bedeutungen.

Generell betrachtet wäre dies der Punkt, wo eine interkulturelle Betrachtungsweise doch ihre Berechtigung findet. Dies spiegelt sich auch in der Bezeichnung des literaturwissenschaftlichen Diskurses, der zumeist als Interkulturelle und seltener als Transkulturelle Germanistik bezeichnet wird, weil die Sprache der literarischen Texte, mit denen sie sich beschäftigt, eben Deutsch ist, auch wenn das, was in den Texten verhandelt wird, kulturell komplexer, mithin hybrid, also transkulturell sein mag. So bleibt das sprachliche Medium als Residuum eines essenzialistischen Verständnisses von Sprache resp. Kultur hier relevant für eine eher interkulturelle Sichtweise. Hinzu kommt, dass für alle Angehörigen der sprachlich-kulturellen Mehrheitsgesellschaft, die tendenziell eher monolingual sozialisiert wurden, als Adressaten dieser Literatur der interkulturelle Ansatz die zutreffende Beschreibung darstellt, weil es bei ihnen enge Grenzen des fremdsprachigen und -kulturellen Verstehens geben wird. So zeigt sich zwar mit Welsch, dass logisch mit dem interkulturellen Modell ein vitiöser Zirkel verbunden ist. 
Gleichwohl ist dieser für das Verstehen literarischer Texte im gegebenen Zusammenhang hermeneutisch notwendig.

Dies wird auch deutlich, wenn man in einer Engführung von Hermeneutik und Komplexität die in beiden Kontexten wichtige Teile-Ganzes-Relation aus der Mereologie mit einbezieht. ${ }^{8}$ Will ich den literarischen Text als Bestandteil einer kulturellen Formation verstehen, so muss ich das Ganze, den kulturellen Hintergrund kennen, muss mit ihm vertraut sein, damit es zu einer Verschmelzung der Horizonte von Text und Leser kommen kann. Wenn nun aber der literarische Text Elemente enthält, die aus einer fremden Kultur stammen, müssen vom Leser Bezüge hergestellt werden, die den Kontext der eigenen Kultur transzendieren. Wie gesehen, ergibt sich dabei als Gradmesser für die systemische Komplexität nicht nur die Zahl der im Text verwendeten Elemente (Figuren, Schauplätze, Zeitebenen, Motive usf.) und das Maß der Verknüpfungen zwischen ihnen, sondern es kommen vom Rand her, von den Positionen des Autors wie des Lesers Aspekte hinzu, die eine rein textimmanente Betrachtungsweise zu verkürzt erscheinen lassen. Geht man rein hermeneutisch davon aus, dass jedes Verstehen, sei es textbezogen oder nicht, sich strukturell über die Relationierung der Teile zu einem Ganzen vollzieht ${ }^{9}$ (also anders als bei Welsch nicht nur durch einen konnektionistischen Anschluss an einen global wirksamen Prozess), so müsste man das Ganze, den Hintergrund der eigenen Kultur, mereologisch gesehen eben dadurch erweitern, dass zunächst fremde Elemente hinzugefügt und akkulturiert werden können. Da das Medium, in dem dies geschieht, ja die deutsche Sprache bleibt, sollten interessierte Leser aus der Mehrheitsgesellschaft damit keine größeren Schwierigkeiten haben. Die größere Leistung bei der Bewältigung des komplexen Szenarios zwischen fremder und eigener, Herkunfts- und Zielkultur dürfte auf Seiten der Autorinnen und Autoren liegen, die als Grenzgänger im Chaos der real gegebenen, sprachlich-kulturellen Komplexität mithilfe der Literatur Brücken bauen, die sich auch für die monolingualen Mitglieder der Mehrheitsgesellschaft als tragfähig erweisen. Nicht zuletzt kann es ihnen auf diese Weise gelingen, am kulturellen und damit sozialen Leben der Zielkultur teilzunehmen, ja vielleicht sogar einen

8 Mereologie ist die Lehre vom Teil und vom Ganzen.

9 Vgl. dazu Rüdiger Ahrens: »Hermeneutik«, in: Nünning (Hg.), Lexikon Literatur- und Kulturtheorie (2001), S. 244-247, hier besonders S. 246. 
Platz im zunehmend interkulturell werdenden Gedächtnis der Kulturnation einzunehmen. ${ }^{10}$

10 Vgl. zum neuen Begriff des interkulturellen Gedächtnisses Dominik Zink: Interkulturelles Gedächtnis. Ostwestliche Transfers bei Saša Stanišić, Nino Haratischwili, Julya Rabinowich, Richard Wagner, Aglaja Veteranyi und Herta Müller, Würzburg: Königshausen \& Neumann 2017, S. 36-41. Am Beispiel der genannten Autorinnen und Autoren untersucht Zink Erinnerungsphänomene, die bisher von der transnationalen bzw. transkulturellen Erinnerungsforschung nicht erfasst wurden und daher »drohen zwischen den Kontexten - zwischen den Kulturen - verloren zu gehen.« Ebd., S. 41. Auch in seiner Arbeit geht es, wie in der vorliegenden Studie, um Zusammenhänge, die bisher in der Forschung noch kaum erfasst wurden. 



\section{Narration in Echtzeit - Komplexes Erzählen in Navid Kermanis Roman Dein Name}

Navid Kermanis Dein Name ist nicht nur ein Roman der interkulturellen Literatur, bei der die interne Transkulturalität, sprich Komplexität, der erzählten Figuren eine wichtige Rolle spielt, sondern zugleich einer der ersten großen Echtzeitromane in medientheoretischem Sinn, wo das Verhältnis von literarischer Produktion und der dabei real vergehenden Zeit zum Gegenstand des literarischen Erzählens selbst wird. ${ }^{1}$ Verschwindet unter Bedingungen der telematischen Echtzeit, wie Paul Virilio sagt, das Intervall, also die Strecke zwischen der Erzeugung/Sendung des Signals und seiner Ankunft, ${ }^{2}$ so nähern

Vgl. dazu den Anfang des Romans: »Es ist Donnerstag, der 8. Juni 2006, 11:23 Uhr auf dem Laptop, der einige Minuten vorgeht, also 11:17 Uhr ungefähr oder, da er den Satz noch schreibt, 11:18 Uhr.« Navid Kermani: Dein Name [2011], 3. Aufl., Reinbek: Rowohlt 2015, S. 7. Indirekt macht Kermani selbst in seinen Frankfurter Poetik-Vorlesungen darauf aufmerksam, wenn er konstatiert: „Das natürliche Medium, die Welt in Unordnung zu erfassen, wie sie in unsere Wahrnehmung tritt, scheint das Internet zu sein, das Schreiben in Echtzeit ermöglicht. « Navid Kermani: Über den Zufall. Jean Paul, Hölderlin und der Roman, den ich schreibe. Frankfurter Poetikvorlesungen, München: Hanser 2012, S. 32. Bereits Rainald Goetz hat in seinem Roman-Tagebuch Abfall für alle, zunächst als Internet-Blog geführt und dann 1999 als Buch mit dem Untertitel Roman eines Jahres publiziert, mit diesem Verfahren experimentiert.

2 Zum Verschwinden des raumzeitlichen Intervalls zugunsten des Intervalls der Art Licht vgl. Paul Virilio: Fluchtgeschwindigkeit, 2. Aufl., Frankfurt a.M.: Fischer 2001, S. 2429. Folgt man Virilios Überlegungen, so wird unter Bedingungen telematischer Echtzeit das Raum- und Zeitintervall der Chronologie zugunsten der Chronoskopie (Zeit der unmittelbaren Belichtung) überschritten, bei der das räumliche Intervall, die Strecke, zugunsten eines ubiquitären Jetzt verschwindet. Es »kommt zur Entstehung einer wirklich paradoxen Kultur, in der alles ankommt, ohne daß es notwendig wäre, sich physisch fortzubewegen, oder überhaupt nur wegzugehen.« Ebd., S. 33. Das der Bewegungslosigkeit hingegebene Subjekt, das seine Realitätserfassung an Bildschirme, Sonden und Detektoren delegiert hat, ist mental nicht länger im Hier und Jetzt erleb- 
sich im literarischen Text die Zeit des Erzählens/Erzählzeit (Zeit, die für die Genese des Signals/Texts vonnöten ist) und die erzählte Zeit weitgehend einander an, ja werden tendenziell identisch mit dem Effekt, dass die Zeit sich zusammenzieht auf den Punkt der instantanen Gegenwart, des augenblicklichen Jetzt, in dem die erzählte Figur namens Navid Kermani (NK) lebt und den Roman schreibt, den der Leser als abgeschlossenes Werk bereits vor sich hat. Dieser performative Widerspruch wird im Text entfaltet und erscheint zugleich als eigentliche Quelle der enormen Produktivität eines Autors, der im »Roman, den ich schreibe «, ${ }^{3}$ wie es im Text immer dann heißt, wenn die Perspektive von der 3. in die 1. Person wechselt, als Fingerzeig darauf, dass die innerdiegetische Figur und der extradiegetische Autor vom Verfasser als tendenziell identische Identitäten angelegt sind, als sein eigener Alter ego auftritt. Dem Text gelingt es auf diese Weise so zu tun, als werde der Leser Zeuge der langwierigen Entstehung eines Romans, bei dem nicht von vornherein klar ist, dass es sich überhaupt um einen Roman handelt, ${ }^{4}$ der jedoch in diesem Augenblick in der Welt des Lesers bereits als fertiges Produkt vorliegt. Der Leser nimmt dabei die erzählte Welt aus der Perspektive der Hauptfigur NK wahr (interne Fokalisierung), ${ }^{5}$ dessen Identität mit dem realen, extradiegetischen Autor gleichen Namens, schränken wir es ein, zumindest nahegelegt wird.

Die genannten Eigenschaften sind zugleich Merkmale autofiktionalen Erzählens, das, neben der rein technischen Beschreibung als metaleptischer

ter phänomenaler Gegenwart präsent, sondern abwesend im virtuellen Raum eines kommutativen Anderswo. Ein solches Subjekt wäre, zugegeben, allerdings kaum mehr ein Leser, vielmehr ein an Fernwahrnehmung und Fernsteuerung interessiertes Wesen. Die (Wahrnehmungs-)Daten mit denen es beschäftigt ist, wären auch keine ZeichenBedeutungen (Sinnträger der Schrift) im herkömmlichen Sinn mehr, sondern vielmehr Signale bzw. Positionen innerhalb der Steuerungsmechanismen von Prozessströmen.

3 Während es zunächst noch heißt: »Er muß sich beeilen. Ich will den Roman nicht schreiben[...]«, (ebd., S. 10) bekennt sich der Erzähler wenige Zeilen später: »Davor ist zu bedenken, ob der Roman, den ich also schreibe, auch Djavad Ketabi bedenkt [...]«. Ebd., S. 11.

»Wüßte er bereits, daß er einen Roman schreibt [...].«N. Kermani: Dein Name, S. 8. Zunächst handelt es sich von der Schreibintention eher um tagebuchartige Aufzeichnungen, die erst sehr spät im Zusammenhang der Verhandlungen mit dem Verlag zu einem Roman werden. Darauf hat bereits Swen Schulte Eickholt hingewiesen. Vgl. Ders.: »Dein Name«, in: Michael Hofmann/Klaus von Stosch/Swen Schulte Eichholt, Navid Kermani, Würzburg: Königshausen \& Neumann 2019, S. 103-130, hier S. 103.

5 Zur internen Fokalisierung vgl. M. Martinez/M. Scheffel: Erzähltheorie, S. 63-66. 
Grenzüberschreitung zwischen extra- und intradiegetischem Raum, wieder im Sinne einer Fusion mentaler Repräsentationen mit resultierenden emergenten Wirkungen beschreibbar ist. Nämlich als Verschmelzung der beiden ontologisch verschiedenen Bereiche der Fiktion (innerdiegetisch) mit der Realität des Autors Navid Kermani (extradiegetisch) im Bewusstsein des Lesers zu einer höherstufigen, d.h. komplexeren Vorstellung von Wirklichkeit, die um den Anschluss an die Wirklichkeit des Rezipienten erweitert wäre. Hinzu kommt nun, dass der Leser, wie angedeutet, sozusagen unter Bedingungen der Echtzeit, über den Fokus des mitlaufenden Gegenwartspunktes (der Erzählung), der sich analog einer Trajektorie (Weltlinie) ${ }^{6}$ im raumzeitlichen Kontinuum der erzählten Welt als unvorhersehbarer Prozess entfaltet, am Alltagsleben des Protagonisten NK teilnimmt. Der Eindruck enormer zeitlicher Verdichtung des erzählten Geschehens ist dabei, neben der überbordenden Fülle der täglichen Aktivitäten des Protagonisten, das Resultat der Auflösung herkömmlicher, zeitlicher Chronologie. Die zeitlichen Modi Vergangenheit und Zukunft werden in einer sich endlos (aus-)dehnenden Gegenwart aufgelöst und so in löslichem Zustand, gewissermaßen als Surrogat-Extrakte, frei verfügbar. ${ }^{7}$ Die Zukunft, die sich real ja der menschlichen Bestimmung in

6 Gemeint ist die Bahnkurve eines Körpers in einem (komplexen) physikalischen System, im erweiterten Sinn eine Kurve im n-dimensionalen Phasenraum.

7 Auch Paul Virilio spricht mit Bezug auf das von den Postmodernisten verkündete Ende der Geschichte davon, dass »die Augenblicklichkeit und die Allgegenwart die uralte Dreiteilung von Vergangenheit-Gegenwart-Zukunft eliminieren. «Paul Virilio: Der Futurismus des Augenblicks, übers. aus d. Franz. von Paul Maercker, Wien: Passagen Verlag 2010, S. 47. Auf diese neue Erfahrung von Zeit nimmt auch Vilém Flusser Bezug. Wie er schreibt, bekommen die Zeitindizes unter Bedingungen zunehmender Digitalisierung eine neue Bedeutung. Sie werden relativ. Vgl. V. Flusser: Die Schrift, S. 132. Kommt es mit Virilio zum Verschwinden des raumzeitlichen Intervalls zwischen Sender und Empfänger, so bestätigt Flusser im Grunde durch seine Überlegungen zur veränderten Wahrnehmung von Zeit, was als alternative Chronologie am Beispiel von Custafssons Roman diskutiert wurde und als Möglichkeit der Zeitumkehr in Kluges Ceschichte und Eigensinn noch zu erörtern ist. In dem Moment, wo das historische Bewusstsein, das von linearer Sukzession bestimmt war, vom Modus digitaler Simulation abgelöst wird, kommt es auch zu einer Umkehr überkommener, zeitlicher Abfolge gemäß Vergangenheit, Gegenwart und Zukunft. Dazu schreibt er: "Zwar mag uns die Relativitätstheorie beim Erlernen dieser neuen Bedeutung behilflich sein, aber wir müssen sie existentialisieren. Versuchen wir das, dann sind wir weniger vom Verschlucken des Raums durch die Bilder und mehr von der Umkehrung des Zeitablaufs beeindruckt: nicht mehr aus der Vergangenheit zur Zukunft, sondern aus der Zukunft der Cegenwart entgegen.«Ebd. 
wesentlichen Hinsichten entzieht, wird im progressiven Immer-nur-weiter des gegenwärtigen Erzählmoments als Richtung der erzählerischen Bewegung operationalisiert bzw. in den kurzfristigen Planungen von Terminen des Protagonisten wie Lesungen, Radiointerviews und Fernsehauftritten, Besuchen bei Freunden und gemeinsamen Unternehmungen mit der Familie usf. antizipiert. ${ }^{8}$ Die Vergangenheit indessen erscheint in Form von Elementen aus der Lebensgeschichte/Biografie des realen, empirischen Autors Navid Kermani, der autobiografischen »Selberlebensbeschreibung «" seines Großvaters und der eingestreuten Nekrologe befreundeter Intellektueller. ${ }^{10}$

Damit kann, indem Geschehen und Begebenheiten aus dem Leben des realen Autors in den Prozess der autopoietischen Genese der literarischen Fiktion einfließen, in analoger Weise wie in ANHs Roman Buenos Aires. Anderswelt die Nullstelle des Erzählens in den Text migrieren und durch Reflexion des Diskurses auf sich selbst Wahrnehmbarkeit erst erheischen. Es kommt im Sinne des Blendings wiederum zu einer Fusion verschiedener Komponenten im Raum der literarischen Erzählung. Daher rührt zu weiten Teilen der Eindruck der Überfülle des Romans, bei der ein Ereignis das nächste jagt, eine Begebenheit sich an die folgende anschließt und es immer wieder Probleme mit der Überwindung räumlicher Distanzen zwischen den vielen Terminen und Verabredungen gibt. Einer Überfülle, die von fern an das Barock erinnern mag, jedoch der überwältigenden Komplexität einer erlebten Gegenwart unter Echtzeitbedingungen geschuldet ist. Der Eindruck der nahezu pausenlos kontinuierlichen Aktivität des Protagonisten verdankt sich dieser Verflüssigung der zeitlichen Ebenen im Fusionspunkt des erzählenden Bewusstseins,

Vgl. dazu S. Schulte Eickholt: »Dein Name«, in: Hofmann/v. Stosch/Schulte Eickholt, Navid Kermani (2019), S. 108: »Dein Name ist im Präsens erzählt, was Unmittelbarkeit generiert und den Roman der Zukunft öffnet [...].«

N. Kermani: Dein Name, S. 33. Vorbild ist dabei die gleichnamige Autobiografie von Jean Paul, der in seiner Konjektural-Biographie mit dem Titel Selberlebensbeschreibung Jugenderinnerungen notiert hat. Auch bei ihm geht es um die Frage nach dem Ich, vor allem darum, wie es sich für den Schreibenden als Innenraum im Unterschied zur äußeren Welt darstellt. Kermani bezieht sich an vielen Stellen im Roman auf das Werk von Jean Paul, weil es in ähnlicher Weise die Beziehung zwischen Schreiben und Leben reflektiert, wie es der Protagonist in Dein Name tut.

10 Diese beginnen mit dem Nekrolog für István Eörsi (ebd, S. 16-19) und setzen sich bis zum letzten Eintrag für Mohammad Mehriar (ebd., S. 1214-1217) verstreut über das ganze Werk hindurch fort. 
dessen Gegenwart im Roman als räumliche Kontiguität der im Text aufeinander folgenden, alltäglichen Begebenheiten tendenziell endlos entfaltet wird. Dies mag Rezensenten, wie Andreas Isenschmid, dazu bewogen haben, im Fall von Dein Name von einer Verwandlung der eigenen "Weltwahrnehmung " ${ }^{11}$ $\mathrm{zu}$ sprechen. Zweifellos handelt es sich dabei um eine Spielart literarischen Schreibens unter den Echtzeitbedingungen der Postmoderne, die nicht zuletzt mit der Verwendung digitaler Schreibgeräte und Textverarbeitungsprogramme zu tun hat, wie sie auch im Roman vorkommen. So ist der schreibende Protagonist ständig mit einem Laptop unterwegs, auf dem nicht nur der Roman als Work in progress entsteht, dessen Arbeitstitel deshalb von Zeit zu Zeit wechselt und der im Moment der Gegenwart der erzählten Welt nur in Form eines offenen, unabgeschlossenen Prozesses mit vielen Möglichkeiten der Fortsetzung existiert. Der portable Computer dient ihm auch als EchtzeitKommunikationsmittel (Email), als Zeitgeber und, spätestens seit dem Roman Kurzmitteilung (2007) ist dies bereits so, auch als Planungsinstrument, über das die Welt mit ihm und auch sein Text potenziell mit der Welt vernetzt ist.

Dass im Roman der Prozess seiner Genese simulierbar wird, verdankt sich folglich, wie erläutert, dem Differenzial des Davor/Dabei und Danach, der prozesshaften Wirklichkeit der Diegese bzw. der vom Autor zurückgelegten (Zeichen-)Strecke auf der einen Seite und der performativen Reproduktion des darin enthaltenen zeitlichen Paradoxes durch den Rezipienten andererseits. Dies ist zudem der Grund dafür, warum Kermanis Roman keine Handlung etwa im Sinne realistischen Erzählens entfalten kann. So wie der Protagonist sein Schreiben deskriptiv zu bestimmen und zugleich zu begründen sucht, handelt es sich um eine Aktivität, die eng mit der eigenen Wahrnehmung der Umgebung sowie den daran gebundenen Gedanken- und Vorstellungsinhalten zusammenhängt. Angesichts des selbst formulierten Vorwurfs der Hybris präzisiert er diese Überlegung:

»[...] ich nehme es natürlich ganz anders wahr und interessiere mich gar nicht für mich, sondern schreibe nur auf, was vor und hinter meinen Augen an mir vorüberzieht, und die Anmaßung, die ich zugebe, liegt nicht darin, daß ich mich selbst betrachte, [...] ... die Anmaßung liegt darin, daß ich von mir 
aus - aber von wo sonst?-, nur von mir aus am Schreibtisch vor dem Computer und den vielen Büchern eine ganze Welt sehe, daß ausgerechnet von mir aus die ganze Welt betrachtet werden soll. Die Welt wäre genauso vollständig von jedem anderen Ich aus zu betrachten, daß glaube ich ganz fest, nur daß die wenigsten Bericht erstatten.«

Ein Stück zuvor gibt er zu Protokoll:

»Ich selbst schreibe seit vier Jahren einen Roman, der nichts anderes tut, als meine Gegenwart gegen die Zeit zu imprägnieren und wenn schon nicht die, wenigstens eine untergesunkene Welt aus dem Meeresboden der Vergessenheit heraufzuholen $[\ldots] \ll{ }^{12}$

Dass sich ein Schreiben, welches sich derart als nicht abschließbarer, in der Gegenwart verwurzelter, nach vorn offener Prozess versteht, nicht im Sinne einer geschlossenen Handlung oder Geschichte gestalten lässt, ist leicht nachvollziehbar. Zudem kann ein solcher Schreibansatz, sofern eine ganze Welt im Text erscheinen soll, eher als ultimativer Versuch des Strebens nach epischer Totalität verstanden werden, darin den Vorgängern Vargas Llosa und Doderer vergleichbar, wenn auch unter anderen Bedingungen, nämlich denen der telematischen Echtzeit. Wenn es sich dabei um den Versuch eines Menschen handelt, der am Schreibtisch sitzend eine ganze Welt sieht und dessen ganzes Handeln darauf gerichtet ist, diese innere Weltwahrnehmung in Form des entstehenden Romantextes zu verschriftlichen, so wird auf diese Weise begreifbar, was Kermani meint, wenn er an vielen Stellen des Romans davon spricht, er wolle ein Gedächtnis verrichten, ${ }^{13}$ Bericht erstatten, Zeugnis ablegen. Ein Gedächtnis wäre damit nichts Gegebenes, auf das sich jederzeit erinnernd zurückgreifen ließe, sondern etwas, das in der Arbeit des Schreibens erst noch zu verrichten ist. Wenn zudem Hinweise auf die unterschiedlichen Bearbeitungsstufen im Text selbst gegeben werden, ${ }^{14}$ so deutet dies von neuem auf das unabschließbar Transitorische des gesamten Projekts hin. Zusammen mit der Erfahrung der Kontingenz des im Alltag des Protagonisten begegnenden Geschehens, das ebenso gut, gerade in den geschilderten Details, auch anders hätte verlaufen können, ohne dass sich dadurch an der

\footnotetext{
12 N. Kermani: Dein Name, S. 1121.

13 Vgl. ebd., S. 9 und 10.

14 Vgl. S. Schulte Eickholt: »Dein Name«, in: Hofmann/v. Stosch/Schulte Eickholt, Navid Kermani (2019), S. 111.
} 
Weltwahrnehmung wesentlich etwas ändern würde, resultiert daraus als ästhetische Wirkung der Eindruck großer Authentizität, ja sogar die Evidenz, dass, anders herum, Authentizität, gar Wahrheit, als Effekte dieser Form der Darstellung verbucht werden können.

Wenn es in der unabgeschlossenen Prozessphase der Textentstehung real je unterschiedliche Möglichkeiten der Fortsetzung gibt (»Wüßte er bereits, daß er einen Roman schreibt, würde er an dieser Stelle eine Affäre erfinden. Noch ist Gelegenheit[...] «), ${ }^{15}$ so manifestiert sich darin die Möglichkeit alternativer Chronologien, die bisher weitgehend dem Bereich der Rezeption vorbehalten blieb. Hier zeigt sich nun, dass dieser Aspekt des kreativen Umgangs mit dem literarischen Plot durchaus auch produktionsästhetisch relevant ist, indem nämlich verschiedene Grade der Bewusstheit, worum es sich bei der eigenen Arbeit eigentlich handelt, d.h. als sich ergebende Stufen von Emergenz innerhalb des kreativen Schreibprozesses, von der erzählenden Instanz vorgeführt werden. Nicht zuletzt ist es gerade dieser Einblick in das Schreibgeschehen, in die Werkstatt des Schreibers, die zugleich der erste Schauplatz des Romans ist, wodurch der Eindruck von Authentizität des erzählten Geschehens insgesamt verstärkt wird. Wenn der Leser, wie erörtert, den Vorgang der Textgenese, zumindest in Ausschnitten, miterleben darf, so weist dies auf eine grundlegende Tendenz im Verhältnis von Realität und Fiktion hin, die für die zunehmende Beliebtheit autofiktionaler Schreibweisen gerade auch in der deutschsprachigen Gegenwartsliteratur ausschlaggebend sein mag. Immer mehr scheinen sich die beiden Bereiche ununterscheidbar ineinander zu schieben, mindestens zu überlappen, sodass auf diese Weise eine Grauzone entsteht, in der niemand zu sagen weiß, ob es sich bei einer Aussage, einer Nachricht, einem Bild um eine authentische Information (Fact) oder um eine Erfindung/Fälschung (Fake) handelt.

Dazu mag, vielleicht weniger als vermutet, die Berichterstattung in den Medien selbst beitragen, die sicher in den meisten Fällen seriös ist, sondern ein viel grundlegenderer, medientheoretisch begründeter Zweifel an der Möglichkeit authentischer Darstellung von Wirklichkeit durch Medien generell. So lautet die Kritik Jean Baudrillards an der damit verbundenen Realismus-Vorstellung, dass die Wirklichkeit heute selbst hyperrealistisch 
geworden sei. ${ }^{16}$ Real ist damit "nicht nur das, was reproduziert werden kann, sondern das, was immer schon reproduziert ist. ${ }^{17}$ Unsere Wahrnehmung von Realität - und darauf kommt es an, es geht nicht mehr um Realität als solche (absolute Realität) - ist, mit anderen Worten, immer schon eine medial reproduzierte. Dies hat zur Folge, dass die Realität im Raum reiner Immanenz mit ihrem eigenen Bild verschmilzt. Die Differenz zwischen Gegenstand und Bild, Realität und Repräsentation der Realität sowie die ursprünglich damit verbundene Vorstellung von Wahrheit und Authentizität verschwindet zugunsten dessen, was Baudrillard Hyperrealität nennt. ${ }^{18}$ Folgt man der Kritik, dass das Hyperreale nicht nur den Widerspruch zwischen dem Realen und dem Imaginären, sondern auch die Differenz zwischen Realität und Fiktion auslöscht, so müsste man sich auch der Konklusion anschließen, dass die Kunst im Grunde tot und die Realität nurmehr in ihre eigene mediale Wiederholung eingeschlossen sei. Umgekehrt resultiert daraus jedoch eine Ästhetisierung des Realen, wenn innerhalb einer künstlich verdoppelten Realität nicht mehr zwischen dem Gegenstand und seiner Repräsentation unterschieden wird. Die Simulation des Hyperrealen kann so zur neuen Form von Wirklichkeit erklärt werden, die ihrerseits im Kern gänzlich von einer medientechnisch strukturierten Ästhetik geprägt ist.

An das auf diese Weise medial vermittelte Bild von Realität, Baudrillard selbst spricht auch von ihrer "ästhetischen Halluzination «, ${ }^{19}$ lässt sich nun die weitergehende Kritik anschließen, dass besonders die audiovisuellen Medien, sofern sie Wirklichkeit auf bestimmte vorgegebene Formate und damit auf bestimmte Sichtweisen reduzieren, Realität in ihrer Komplexität, etwa hinsichtlich der Frage, wie alles mit allem zusammenhänge, nicht gerecht $\mathrm{zu}$ werden vermögen. Gerade die audiovisuellen Medien Fernsehen oder Video, können immer nur einen Ausschnitt der Realität präsentieren, indem sie ein Fenster (Bildschirm) öffnen, das zwar vorgibt repräsentativ zu sein, es in einem umfassenden Sinn aber nie sein kann. Dieser Zweifel schlägt in einer weitgehend mediatisierten Gesellschaft auf die Wahrnehmung von Wirklichkeit insgesamt durch und lässt das, was man zu sehen und $\mathrm{zu}$

16 Vgl. Jean Baudrillard: Der symbolische Tausch und der Tod, übers. aus d. Franz. von Gerd Bergfleth, Gabriele Ricke und Ronald Vouillié, München: Matthes \& Seitz 1991 , S. 116.

17 Ebd.

18 Vgl. dazu ebd., S. 112-119.

19 Ebd., S. 116. 
hören bekommt als partikular, ja als Schwundstufe eines technischen Versprechens von höherer Allgemeinheit (gemäß dem Prärogativ der visuellen Wahrnehmung) erscheinen.

Der Literatur hingegen gelingt es umgekehrt, durch Aktivierung des Imaginationsvermögens der Leser (Iser) und weiterer Formen der Partizipation (Hiergeist) das Erlebnis einer intellektuell umfassenderen Realitätserfahrung zu ermöglichen, das sich nicht auf die programmierten Reaktionen einer konnektionistischen Medientechnik reduzieren lässt. Gerade weil die Potenziale des Schriftzeichens und der narrativen Struktur auf die Aktivität des Lesers angewiesen sind, können im literarischen Text an sich getrennte bzw. weit voneinander entfernte Bereiche von Realität eine Verbindung eingehen, sodass selbst das in der realen Wirklichkeit Unwahrscheinlichste, gar Unmögliche in der Welt der literarischen Erzählung zutreffend sein kann. Zwar hat die Literatur die grundlegenden Anschauungsformen von Raum und Zeit (Ausgedehntheit und Folge), der Kausalität, der Prinzipien von Identität und Differenz sowie der semiotischen Strukturen von Sinn und Bedeutung für die Konstruktion narrativer Wirklichkeit(en) übernommen, doch geht es dabei nicht um ihre Wiederholung als Selbstzweck, ${ }^{20}$ sondern vielmehr darum, diese Kategorien im Raum der Fiktion für neue Möglichkeiten der Weltwahrnehmung durchlässig zu machen. Davon sind auch veristische Strömungen wie der Nouveau roman, der Neue Realismus oder die Popliteratur nicht ausgenommen. Schon daraus ergibt sich, dass die Literatur im Grunde von jeher daran interessiert war, die Grenze zwischen Realität und Fiktion ästhetisch, also im Hinblick auf Prozesse der Wahrnehmung in der ambivalenten Interaktion zwischen Text und Leser, durchlässig zu machen. Eine Strategie, die auch für die Autofiktion typisch ist, sofern sich die Autoren dieses Genres als Figur oftmals mit gleichem Namen, wie im Fall von Dein Name, in ihren eigenen Text einschreiben und den irritierten und dadurch aktivierten Leser damit vor die unentscheidbare Frage stellen, ob es sich um einen autobiografisch-veristischen oder um einen fiktionalen Text handelt.

Vor diesem Hintergrund lässt sich nun sagen, dass dies auch als Antwort auf eine zunehmend komplexer werdende Wirklichkeit verstanden werden kann, indem nämlich der Autor einräumt, dass er die eigene Identität keineswegs länger als ausnahmslos kohärent und kontinuierlich erlebt, sondern

20 Iser spricht auch davon, dass das Reale in einem fiktionalen Text keinen Selbstzweckcharakter hätte, sondern als »Zurüstung eines Imaginären« fungiere. W. Iser: Das Fiktive und das Imaginäre, S. 18. 
eher zerfällt in verschiedene Zustandsformen, die situativ gebunden oder, wie bei ANH, fraktal gebrochen auftreten. So erklärt sich nicht nur der Wechsel der Perspektive, der mit dem Switch der Pronomen von der 1. in die 3. Pers. Sg. und vice versa verbunden ist und in analoger Weise sowohl bei ANH als auch bei Kermani zu finden ist, durch eine Öffnung personaler Identität hin zu Alterität, tendenziell Pluralität, sodass der Blick des/der anderen auf sie selbst genauso zur Wirklichkeit einer Person gehört, wie die eigene Sicht auf das, was sie sei. Hinzu kommt, dass der Protagonist in Dein Name je nach Aktivität eine andere Selbstbezeichnung wählt. So wechselt nicht nur der Arbeitstitel des Textes (beginnend mit Totenbuch) ${ }^{21}$ von Zeit zu Zeit, auch die Subjektstelle ist je nach Rolle bzw. Tätigkeit unterschiedlich besetzt. Die Palette reicht vom »Romanschreiber, sonst Sohn, Vater, Mann, Freund, Nachbar oder Handlungsreisender, hin und wieder Enkel, regelmäßig Berichterstatter, dann wieder Orientalist, ein Jahr lang die Nummer zehn und an einigen Stellen Navid Kermani ${ }^{22}$ Es sind dies gewissermaßen je alternative Zustände bzw. Möglichkeiten eines identischen Subjekts, das sich in seiner Rolle als Autor des Romans darüber Rechenschaft gibt, wie verschieden es sich selbst in den unterschiedlichen, damit angesprochenen Kontexten als Mensch erlebt hat. ${ }^{23}$ Dass »ich « viele sind und eben nicht nur ein und derselbe, wie es ein essenzialistisches Verständnis des Eigennamens nahelegt, dafür wäre die Öffnung der Perspektive des erzählten bzw. erzählenden Subjekts ein weiteres Beispiel.

Aus dieser an (subjektiver) Wahrnehmung sich orientierenden Schreibhaltung resultiert nun noch ein weiterer Aspekt, der in Hinsicht auf Komplexität relevant ist. Dies betrifft die damit gegebene, enge Verschränkung des Schreibvorgangs mit der Umwelt, die Gegenstand der Wahrnehmung des schreibenden Subjekts ist. Das Schreiben, das als Akt im Milieu der Umwelt situiert ist, speist sich somit weitgehend aus Impulsen und Signalen (bzw.

\footnotetext{
21 Vgl. N. Kermani: Dein Name, S. 12.

22 Ebd., 1113.

23

Ähnlich argumentiert auch Natalie Knapp in einem Cespräch mit Matthias Eckoldt über integrales Bewusstsein. Ihr Vorschlag, das Ich nicht länger als Einheit, sondern im Sinn einer »Wechselwirklichkeit« zu verstehen, resultiert aus der Wahrnehmung, dass sich das Ich in unterschiedlichen Kontexten wandelt, sodass, wie Knapp sagt: »ich in unterschiedlichen Kontexten immer eine andere bin. «»Natalie Knapp über integrales Bewusstsein, das Denken in Analogien und die Traurigkeit des Hundes«, in: Matthias Eckoldt, Kann sich das Bewusstsein bewusst sein? Gespräche mit Dirk Baecker et al, Heidelberg: Carl-Auer 2017, S. 198-216, hier S. 209.
} 
auch Reizen, die Erinnerungsprozesse auslösen), die aus der Umwelt kommend auf das Bewusstsein des Schreibenden einwirken, mit ihm interagieren und auf diese Weise als Taktgeber der Erzählzeit fungieren. Da es sich bei dieser Interaktion mit der Umwelt zumeist nicht um einmalige Ereignisse handelt, sondern um zyklische, d.h. sich wiederholende Muster der Referenzialisierung/Bezugnahme auf Umwelt, sowohl in ihrer Konstanz wie in ihrer transitorischen Veränderlichkeit, erscheint der literarische Text, sofern es ihm wichtige Impulse verdankt, sozusagen als Abfallprodukt des Alltagslebens. Deswegen hat Swen Schulte Eickholt auch von einer »Poetik des Abfalls« gesprochen. ${ }^{24}$

Möchte man den negativ konnotierten Begriff vermeiden, ließe sich auch von einer Poetik der Verwertung im Sinn des Recyclings sprechen. Mit dieser Metapher aus dem Bereich der Abfallwirtschaft, wo man davon ausgeht, dass einmal verwendete Wertstoffe wieder aufbereitet werden können, soll aufgezeigt werden, dass der literarische Text bzw. in seiner Verlaufsform der Vorgang des Schreibens auch als Interiorisierung (Input) der Umwelt durch das schreibende Subjekt verstanden werden kann. Wird die Rückkopplungsschleife bei Iser als Modus von Emergenz begriffen, die sich im weiteren Sinn durch die Hervorbringung von Kultur (Exteriorisierung) ${ }^{25}$ manifestiert, so kann in einem zweiten Schritt der literarische Text als hervorgebrachtes Produkt analog als Output in die kulturelle Umwelt des Literaturbetriebs aufgefasst werden. Paradoxerweise ist dies jedoch, wie im Fall von Kermanis Dein Name, obwohl der Roman sehr erfolgreich rezipiert und sogar mit dem Friedenspreis des Deutschen Buchhandels ausgezeichnet wurde, nicht zwangsläufig mit einer positiven Rückkopplung verbunden. Positiv ist die Rückkopplung nur dann, wenn, wie Iser schreibt, »die eigenen Vorstellungen massiver Veränderung ausgesetzt sind. $\aleph^{26}$ Alles hängt also davon ab, ob neben den Zielvorgaben, in diesem Fall den Erwartungen des Autors ebenso wie den Kalkulationen des Verlegers, den Vorstellungen des Schreibenden hinsichtlich der ästhetischen Wirkungen durch den Literaturbetrieb bzw. die Aufnahme der Leser widersprochen wird oder nicht. Auch wenn, wie im Fall von Dein Name,

24 Swen Schulte Eickholt: »Über den Zufall. Jean Paul, Hölderlin und der Roman, den ich schreibe«, in: Hofmann/v. Stosch/Schulte Eickholt, Navid Kermani (2019), S. 131-150, hier S. 137 und 143. Vgl. dazu auch N. Kermani: Über den Zufall, S. 47. Auf diesen Text bezieht sich Eickholt mit seiner Wendung.

25 Zum Begriff Exteriorisierung vgl. W. Iser: Emergenz, S. 69.

26 Ebd., S. 72. 
die Erwartungen weit übertroffen werden, das Buch von Kritik und Leserschaft, die Verkaufszahlen können das belegen, als schöner Erfolg gewertet wird, kann die Rückkopplung für den Autor in dem Fall negativ ausfallen, wo er durch die literarischen Öffentlichkeit - im Rahmen von Lesungen oder Fernseh- und Radiointerviews usf. - in seinen Vorstellungen sich nurmehr bestätigt sieht, weil daraus kein Anreiz zu einer neuerlichen Reformulierung seiner Schreibweise/Poetik resultiert. Der Prozess setzt sich jedoch, wie auch im Roman deutlich wird, zumeist ohne Rücksicht auf professionelle Befindlichkeiten des Autors fort, indem jede publizistische Verlautbarung zum Anlass zahlreicher Interventionen seitens der Kritik, der Medien und der Literaturwissenschaft wird, die dem Schreiber seinerseits als Input für weitere Einlassungen dienen. So gesehen lässt sich sagen, dass es durch den literarischen Text komplexitätstheoretisch zu einer Kultivierung von Entropie in der kulturellen Umwelt kommt. Bericht erstatten, Zeugnis ablegen, ein Gedächtnis verrichten wären, mit anderen Worten, auch als Versuche des Autors zu werten, dem entropischen Verschwinden nicht nur geliebter und befreundeter Menschen, sondern auch der eigenen Lebensgeschichte, den damit verbundenen, interkulturellen Beziehungen und der kulturellen Landschaft bundesrepublikanischer Gegenwart der ausgehenden Nullerjahre, besonders seiner Heimatstadt Köln, im Rahmen des Strebens nach epischer Totalität zu begegnen.

Komplexitätstheoretisch ist zudem die in der Sekundärliteratur und auch von Kermani selbst ausgiebig reflektierte Kontingenz des Romangeschehens wesentlich. ${ }^{27}$ Dabei ist von entscheidender Bedeutung, dass das Bildungsgesetz des literarischen Textes erst im Verlauf des Schreibvorgangs selbst generiert wird bzw. daraus im Sinne von Emergenz hervorgeht. Dies betrifft zunächst den Status des mehr als Aktivität denn Werk verstandenen Schreibens, sofern der Autor selbst sich über weite Strecken im Unklaren ist, ob es überhaupt ein Roman werden kann oder nicht doch nur tagebuchartige Aufzeichnungen bleiben sollen. Dann gilt dies auch für das Ziel, die Finalität des Wozu (bzw. das Warum der Begründung) eines so verstandenen Schreibens, das erst im Verlauf des Prozesses gefunden und als Zeugnis ablegen, Bericht erstatten, ein Gedächtnis verrichten benannt wird. Und schließlich bedeutet die Fokussierung des Erzählers auf das, was ihm im Alltag begegnet und wovon er an den unterschiedlichsten Schauplätzen umgeben ist (Umwelt), eine

27 Vgl. dazu N. Kermani: Über den Zufall, 1. und 5. Vorlesung, sowie den bereits genannten Aufsatz von Eickholt über Kermanis Frankfurter Poetik-Vorlesungen, S. 144-147. 
Öffnung und damit Sensibilisierung für die Zufälligkeit des Wirklichen, aus der paradoxerweise das Strukturprinzip des gesamten Romans hervorgeht. Folgt man Baudrillard, so geht es in der Postmoderne, dem Zeitalter der Simulation, um die Erzeugung von Modellen:

»Nur die Zugehörigkeit zum Modell ergibt einen Sinn, nichts geht mehr einem Ziel entsprechend vor, alles geht aus dem Modell hervor, dem ReferenzSignifikanten, auf den sich alles bezieht, der eine Art vorweggenommener Finalität und die einzige Wahrscheinlichkeit hat. ${ }^{28}$

Dies kann, wie am Beispiel von Kermanis Roman zu sehen ist, soweit gehen, dass selbst das Unvorhergesehene, das, was sich eigentlich jeder Berechnung entzieht, nämlich der Zufall, paradoxerweise zum Modell der literarischen Produktion wird. So kann, indem die Poetik des Textes Teil des Romans wird, in ihn eingeschrieben ist, sein Bildungsgesetz nicht als nachträgliche Metaebene der Betrachtung, als Metatext aposteriori isoliert vom Rest dieser großen Erzählung aufgefasst werden, sondern muss als weitere Komponente innerhalb eines komplexen, mehrstelligen Endhorizonts der Rezeption insgesamt mit einbezogen werden. Dadurch wiederum erhöht sich die Komplexität des Romans, sofern der Autor die Gesetzlichkeit seiner Genese in den gleichen Atemzügen mitliefert wie das übrige Romangeschehen. Zwar erscheint auch die Poetik als separate Publikation unter dem Titel Über den Zufall (2012), während Dein Name bereits ein Jahr zuvor publiziert wird. Doch wird im Roman an vielen Stellen darauf, sei es inhaltlich in Form von Reflexionen über Jean Paul und Hölderlin, die dem »Poetologe[n] ${ }^{29}$ wie es an diesen Stellen heißt, als Vorbilder für das eigene Schreibprojekt dienen, sei es in Hinsicht auf den bevorstehenden Termin der ersten Vorlesung im Rahmen der Frankfurter Poetik-Vorlesungen am 11. Mai 2010 und den damit zusammenhängenden Vorbereitungen, als Parallelgeschehen Bezug genommen. ${ }^{30}$

Es zeigt sich nun aber bei genauerer Überlegung, dass das schreibende Subjekt der ihm begegnenden Zusammenhanglosigkeit und Heterogenität alltäglicher Wirklichkeit nicht dadurch Herr wird, dass es gewissermaßen alles, was es nur wahrnimmt in den Text überträgt. Es bedarf schon an dieser

\footnotetext{
28 J. Baudrillard: Der symbolische Tausch, S. 89.

29 N. Kermani: Dein Name, S. 1021.

30 Im Roman gibt es ab S. 1021 (bis S. 1158) zahlreiche Bezugnahmen auf die PoetikVorlesung, die er am »Pult von Theodor W. Adorno, wie ihn der Einladungsbrief einschüchterte«, halten wird. Ebd., S. 1021.
} 
Stelle einer Selektion von begegnender Realität, die vom Erzähler (schreibendes Subjekt) durch die Reflexion des Wahrgenommenen geleistet wird, die sich dann im Sinne einer Interaktion zwischen der fiktiven Welt des Romans und der empirischen Wirklichkeit des realen Autors Navid Kermani entfaltet. ${ }^{31}$ Es kommt auf diese Weise wieder zu einer Austauschbeziehung diesmal zwischen der fiktiven Welt des literarischen Textes in evolutionis und der textexternen Realität, sofern sie in der Wahrnehmung des Autors während des Schreibprozesses selektiert wird. Wenn, wie Baudrillard sagt, die Ästhetisierung des Wirklichen so weit fortgeschritten ist, dass »das Reale und das Imaginäre zu einer gemeinsamen operationalen Totalität verschmolzen sind «, ${ }^{32}$ ist es folglich für den Rezipienten auch nicht mehr nötig, nachträglich die Differenz wieder einzuführen, die es ermöglichen sollte, zwischen den ontologisch verschiedenen Bereichen des Realen und der Fiktion zu unterscheiden. Dies gilt in gleicher Weise für die Komponenten innerhalb des übergreifenden Horizonts der Rezeption, ${ }^{33}$ die im Prozessstrom des Erzählens so miteinander kombiniert werden, dass ihre Herkunft aus dem einen oder anderen Bereich (textintern oder -extern) für den Rezipienten unkenntlich wird. Gerade dies ist der Grund dafür, dass sie in der Fantasie des Lesers zu neuen Konstellationen von Sinn und Bedeutung zusammentreten können.

Auf diese Weise können, worauf bereits Eickholt hingewiesen hat, die eingestreuten Nekrologe als »Texte des empirischen Autors über reale Personen« ebenso auch wie die Reisereportagen, die sowohl im Roman als auch separat in der Presse erschienen sind, ${ }^{34}$ gleichzeitig $»$ innerhalb und außerhalb des Romans $\aleph^{35}$ sein. Sie bilden, mit anderen Worten, einen »Rahmen«, der

31 Auch Iser spricht in diesem Zusammenhang von der »für jeden fiktionalen Text notwendige[n] Selektion aus den vorhandenen Umweltsystemen«. W. Iser: Das Fiktive und das Imaginäre, S. 24. Der Schauplatz für diesen Akt wäre freilich das kognitive System des Schreibenden, indes dieser mit seiner literarischen Produktion befasst ist.

32 J. Baudrillard: Der symbolische Tausch, S. 118.

33 Vgl. W. Iser: Emergenz, S. 40.

34 So begleitet Kermani im Dezember 2006 als Beobachter ein Kontingent der Bundeswehr nach Afghanistan. Vgl. N. Kermani: Dein Name, S. 137-168. Der Text erscheint als Reportage gekürzt und leicht umformuliert zunächst unter dem Titel »Trostlose Normalität«, in: Neue Zürcher Zeitung. Zeitbilder vom 16.01.2007, S. 311 f. Dann mit der Überschrift »Trostlose Normalität. Mit der NATO in Afghanistan «, in: Kursbuch 167 (2007), Heft 2, S. 1-19. Seitenangabe zit. nach https://www.yumpu.com/de/document/read /33080701/trostlose-normalitat-navid-kermani vom 16.02.2021.

35 S. Schulte Eickholt:»Über den Zufall«, in: Hofmann/Stosch/Schulte Eickholt, Navid Kermani (2019), S. 141. 
»aus der Auseinandersetzung mit Jean Pauls Ordnungskonzepten hervorgegangen ${ }^{36}$ ist. Damit ist Jean Pauls Bestreben gemeint, in seiner Poetik die Heterogenität des Wirklichen nicht aufzuheben und damit zum Verschwinden zu bringen, sondern sie im Gegenteil in der ästhetischen Gestaltung des literarischen Textes zu erhalten, ja hervorzukehren. ${ }^{37}$ So gewinnt Kermanis Roman durch den »Realitätsanspruch ${ }^{38}$ der in ihn eingearbeiteten heterogenen Textsorten ein höheres Maß an Authentizität, als er ohne sie hätte. Es resultiert zudem als emergenter Mehrwert eine verschränkte Rahmenstruktur zwischen Realität und Fiktion, die Eickholt folgendermaßen erläutert: »Die Heterogenität des Wirklichen erscheint gerahmt und geordnet durch die Gestaltung der fiktiven Welt, die Gestaltung und Ordnung erscheint gleichzeitig gerahmt durch die Heterogenität des Wirklichen. " ${ }^{39}$ Wenn dadurch eine ästhetische Wirkung erzielt werden kann, die jede Spielart herkömmlichen, realistischen Erzählens an Authentizität übertrifft, so hängt dies damit zusammen, dass es nicht darum geht, die Wirklichkeit als solche (objektiv), d.h.

Ebd.

37 Wie eng Kermanis Schreiben an Jean Pauls Poetik angelehnt ist, zeigt sich an folgender Textstelle: »Ein Romanschreiber, der an einigen Stellen Jean Paul genannt wird, behauptet, daß der Roman kein Roman sei und die darin auftretenden Personen sämtlich wirklich existierten, auch außer seinem Kopfe. Und sein Argument ist, daß ein Roman auf Wahrscheinlichkeiten beruhe, also einer Ordnung, die Unwahrscheinlichkeiten nur in dem Maße zuließen, daß sie nicht als Regel erschienen. Hingegen in der Wirklichkeit geschähen so viele Zufälle, daß es in einem Roman für unwahrscheinlich gehalten würde und also ausgeschlossen sei. In dem Roman, den ich schreibe, behauptet der Romanschreiber, der an einigen Stellen Navid Kermani genannt wird, daß der Roman kein Roman sei und Jean Paul wirklich unter der Tischplatte gelegen habe, auch außer seinem Kopfe. Und sein Argument ist, daß ein Roman auf Wahrscheinlichkeiten beruhe, also einer Ordnung, die Unwahrscheinlichkeiten nur in dem Maße zuließe, daß sie nicht als Regel erschienen. Hingegen in der Wirklichkeit geschähen so viele Zufälle, daß es in einem Roman für unwahrscheinlich gehalten würde und also ausgeschlossen sei.«N. Kermani: Dein Name, S. 1025. Über die leere Verdoppelung von Jean Pauls Poetik hinaus, zielt Kermanis Verfahren auf deren Reflexion unter Bedingungen der Jetztzeit. Dabei erweist sich, dass in der Echtzeit-Darstellung des Romans der Zufall, anders als in der Realität zugunsten des Wahrscheinlichen, d.h. des Anschlussfähigen, verschwindet, das Verhältnis von Zufall und Wahrscheinlichkeit gemessen an realer Erfahrung sich also gewissermaßen umkehrt. S. Schulte Eickholt: »Über den Zufall«, in: Hofmann/Stosch/Schulte Eickholt, Navid Kermani (2019), S. 141. Ebd. 
so, wie sie (in Wahrheit) wäre, darzustellen, sondern so, wie sie vom schreibenden Subjekt wahrgenommen wird. Die Wirklichkeit erscheint daher nicht homogen, kohärent und kontinuierlich (Reduktion), sondern heterogen, polarisiert, transitorisch und überfordernd in der Komplexität ihrer Beziehungen (Komplexion). Sie ist dadurch eher das Resultat der Anschauungsformen und Erlebnisweisen eines individuellen Subjekts denn eine Totalität, die für alle sozusagen gleich bzw. einheitlich wäre. Eine Voraussetzung, die für herkömmliche Realismus-Definitionen grundlegend ist, sofern ein realistischer Text das Wesentliche einer Epoche, einer sozialen Schicht sowie der Lebensund Arbeitsverhältnisse zu einer gegebenen historischen Zeit zum Ausdruck bringen soll. ${ }^{40}$ Dies alles, zusammen mit den zuvor am Beispiel von Virilio und Baudrillard erläuterten Auswirkungen der Echtzeit auf das literarische Schreiben und seine Rezeption, legt es nahe, von einer Ästhetik der Echtzeit zu sprechen. Damit ist nicht nur der Bezug auf die objektiven, gesellschaftlichen Tendenzen umfassender Digitalisierung und Mediatisierung hergestellt, sondern zugleich die veränderte Conditio des Subjekts aufgrund der mit den genannten Tendenzen verbundenen Erfahrung der Disruption des Wirklichen angesprochen.

40 Realismus wird hier aus heuristischen Gründen als Epochenbegriff bzw. literarisches Programm verstanden. Seit dem lingusitic turn in der Literarturwissenschaft ist natürlich davon auszugehen, dass die Sprache (das sprachliche System) unsere Wahrnehmung von Wirklichkeit steuert und aus diesem Grund von einer Vorgängigkeit der Welt, die im Text mimetisch abgebildet wird, nicht länger gesprochen werden kann. Realismus bezeichnet gemäß diesem Verständnis kein Abbildungsverhältnis mehr, sondern einen mit bestimmten künstlerischen Mitteln erzielten Effekt. Dieser Realismus-Effekt (Effet de réel bei Barthes) meint die von literarischen Texten ausgehende Wirkung, die beim Leser den Eindruck von Wirklichkeitsnähe bzw. Authentizität der in ihnen dargestellten fiktionalen Wirklichkeit hervorruft und nicht eine bestimmte literarhistorisch definierte Epoche. Vgl. Richard Humphrey: »RealismusEffekt«, in: Nünning (Hg.), Lexikon Literatur- und Kulturtheorie (2001), S. 540 f. Dieser Effekt ist fraglos auch für Kermanis Dein Name evident. 


\section{Inwiefern komplexes Erzählen zu einer veränderten Wahrnehmung der Lebenswirklichkeit von Lesern beitragen kann}

Hier nun lässt sich das zu Beginn umrissene Zeitgefühl einer verloren gegangenen Ganzheit, das ausgehend von der durch die Komplexitätsforschung bestätigten Prämisse, dass alles mit allem zusammenhänge, zugleich verknüpft war mit einem umfassenden Erklärungsanspruch und der gefühlten Unfähigkeit, die Dinge zum Besseren zu ändern, epistemisch noch einmal präzisieren. Es geht dabei darum, das Prinzip der alternativen Chronologien auf die Biografie, den Lebenslauf von Subjekten zu übertragen. Auf diese Weise wird ein Verfahren, das in Form der Variantenromane, wie z.B. Dieter Kühns $N$, Éric-Emanuel Schmitts Adolf H. Zwei Leben oder jüngst Paul Austers 4321, bereits aus der erzählenden Literatur bekannt ist, mit einer postmodernen Befindlichkeit des Subjekts in Zusammenhang gebracht, dessen Kern Michael Lommel »als Erfahrung einer Verzweigung von Biographien ${ }^{1}$ beschrieben hat und im Sinn von »Erweiterungen oder Vervielfachungen der Identität mit den Mitteln der Einbildungskraft « versteht. ${ }^{2}$ Da das Subjekt der telematischen Echtzeit, folgt man Virilio, immer weniger dazu in der Lage ist, intensive Präsenzerfahrungen zu machen, obwohl man faktisch betrachtet doch nur im Hier und Jetzt zu leben vermag, bleiben ungelebte Möglichkeiten entweder nur für die Zukunft oder das Imaginäre des Augenblicks in Form von möglichen Lebensvarianten und deren Manifestationen in Literatur und Film. Damit verbindet sich mit Lommel die Frage: »Was wäre, wenn ich an dieser oder 
jener Zeitstelle anders gehandelt, wenn ich anders entschieden oder wenn der Zufall mein Leben in eine andere Richtung gelenkt hätte? «3 Somit belagern Lebensvarianten »die aktuelle Biographie, das gelebte und zu lebende Leben im Wartesaal der Möglichkeiten «, ${ }^{4}$ wie Lommel es nennt.

Auch hier kommt es also zu einer Erweiterung bestehender, biografischer Realität in Richtung einer komplexeren Vorstellung des Selbst, die aus der Potenzialität möglicher Varianten im Vergleich zum faktischen Verlauf einer Biografie resultiert. Das Leben wäre also mehr als nur die eine tatsächlich realisierte Version mit einer begrenzten Zahl von Stationen und dazwischen stattfindenden Veränderungen. Es wäre narratologisch gesprochen eine Art von Mehrfach- oder Parallelerzählung, wo viele nicht realisierte Möglichkeiten das reale Geschehen begleiten, es dabei vielgestaltig bereichern oder vielleicht auch bedrängen, um endlich wirklich zu werden. $\mathrm{Zu}$ bedenken ist dabei wieder, dass der Überschuss an realer Komplexität, die überbordende Fülle an Möglichkeiten und Perspektivendifferenzen innerhalb eines tendenziell globalen Geschehens, mit dem die Subjekte als Mediennutzer verbunden bzw. konfrontiert sind, bedingt, dass es bei dem Versuch, das eigene Leben neu oder anders zu lesen, aus Gründen des Kohärenzaufbaus wiederum zur Reduktion kommt.

Dies gilt umgekehrt auch für das schreibende Subjekt, wenn es bei dem Versuch, das, was es bewegt, worum es ihm bei der Darstellung geht, zur Sprache zu bringen, mit einer »Unendlichkeit sprachlicher Möglichkeiten ${ }^{5}$ konfrontiert ist, die naturgemäß nach Reduktion verlangen. Hinzu kommt, dass dies durch den Akt der Versprachlichung Hervorgebrachte, Peter Sloterdijk vergleicht diesen Vorgang am Beispiel von Emile Cioran mit einer Geburt, ${ }^{6}$ nicht so bleibt, wie es einmal geworden ist, sondern stets vom Möglichen begleitet wird, sofern die einmal so zusammen getretenen Komponenten sich rekombinieren und in weiteren, kreativen Akten zum Ausdruck gelangen. ${ }^{7}$ Wie Koschorke weiß, hängt dieses Abarbeiten an den Möglichkeiten der Sprache damit zusammen, »dass zwischen mentalen Prozessen und ihrer sprachlichen Artikulation, anders als man gemeinhin annimmt,

3 Ebd., S. 166.

4 Ebd., S. 14.

5 A. Koschorke: Wahrheit und Erfindung, S. 28.

6 Vgl. Peter Sloterdijk: Zur Welt kommen - Zur Sprache kommen. Frankfurter Vorlesungen. Frankfurt a.M.: Suhrkamp 1988, S. 106-108.

7 Vgl. W. Iser: Emergenz, S. 82. 
kein Abbildverhältnis besteht. $\aleph^{8}$ Dies hat zur Folge, dass es zu unübersetzbaren Überschüssen auf beiden Seiten kommt, Vorstellungen und Worte also nicht in einem mengenmäßigen Verhältnis der Isomorphie zueinander stehen, ${ }^{9}$ wenngleich davon auszugehen ist, dass sie in irgendeiner Weise zusammen hängen. So kommt es, dass es zu immer neuen Anläufen bei der Bearbeitung eines künstlerischen Problems kommt, das sich durch Umformulierungen, Umstellungen und Überformungen eines zunächst gefundenen Ausdrucks Bahn bricht.

Während Koschorke der Überlegung folgt, dass es sich dabei um einen energetischen Vorgang handelt, der, was die Intensität der Rückkopplung zwischen der Sphäre des Bezeichneten und dem bezeichnenden Subjekt betrifft, mit Analogien aus der Thermodynamik (Hitze/Kälte) beschreibbar ist, ${ }^{10}$ soll es hier um eine für die weitere Analyse wichtige Differenzierung der beiden Modalitäten von Emergenz gehen, die bisher kaum unterschieden worden sind. Nennt Iser die Rückkopplungsschleife, die auch als seltsame Schleife bezeichnet wird, weil die durch sie bewirkte Veränderung eine Rückwirkung der Folge (Bedingtes) auf die Ursache (Bedingung) erlaubt, ${ }^{11}$ eine "Manifestation von Rekursivität «, ${ }^{12}$ so ist damit gesagt, dass Rekursion der allgemeinere Begriff ist. Dazu führt er weiter aus: »Der Kontrast zwischen dem Erzielen von Kontrolle und der Emergenz des Selbsterhalts bringt den Unterschied zwischen einer linear operierenden Rückkopplungsschleife und einer nicht-linear verlaufenden Rekursion zur Geltung. ${ }^{13}$ Während also durch Rekursion der Informationsaustausch zwischen allen Ebenen und Elementen eines Systems in Echtzeit gewährleistet wird, fungiert die Rückkopplungsschleife als Kontrollmechanismus innerhalb eines zweckgebundenen, linearen Prozesses, der über die Wechselwirkung/den Abgleich von Input und Output gesteuert wird. Indem Rekursion durch ständigen, wechselseitigen Informationsfluss zum Erhalt operationell geschlossener Systeme in einer sich verändernden Umwelt beiträgt, moduliert sie zugleich deren Verhaltensmuster in einer Weise, die ihr Überleben sichert. Rekursion ${ }^{14}$ ist also nicht nur wesentlich an der Selbst-

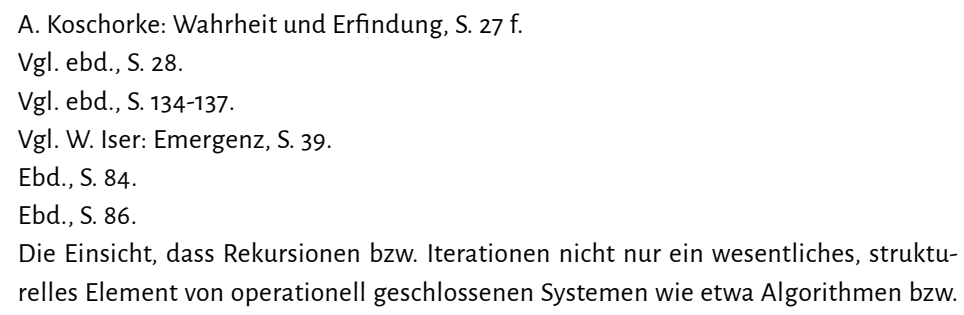


organisation autoreferenzieller Systeme beteiligt, sondern bewirkt zugleich eine beständige Ausdifferenzierung ihrer Verhaltensweisen in der Auseinandersetzung mit der Umwelt.

Um mit Beeinträchtigungen, die von außen einwirken, zurecht zu kommen, bedarf es einer immens großen Zahl von Reaktionsmöglichkeiten, sprich eines Wechsels im Verhalten, der auf die beschriebene Weise durch rekursive Verarbeitung der Störsignale und Neuanpassung des Systemverhaltens zustande kommt. Da dies kein einmaliger Vorgang ist, sondern aus Gründen des Systemerhalts permanent realisiert werden muss, resultiert ein enormes Repertoire von Verhaltensantworten, das sich im Lauf der Zeit weiter ausdifferenziert und insgesamt zur Steigerung der Plastizität des Systemverhaltens beiträgt. Wichtig ist nun, dass eine einmal generierte Verhaltensantwort bei erneuter Umweltänderung und erforderlicher Neuanpassung nicht einfach spurlos verschwindet, also schlicht vergessen wird, sondern in Form »rekursive[r] Geschichten $\aleph^{15}$ nach Varela, d.h. als rekursive Verkoppelungen mit den bereits abgelegten, früheren Verhaltensausprägungen, im System verbleibt. Auf diese Weise kann, je nach Bedarf, gegenwärtiges Verhalten mit systemintern bereits gespeicherten Mustern interagieren, sodass es, ähnlich wie im Roman Buenos Aires. Anderswelt, zu komplex rückgekoppelten Wirk- bzw. Beobachtungszusammenhängen

Programmen sind, sondern auch von literarischen Texten, formuliert Sven Kosub: »lterationen und Rekursionen sind wesentliche operationale Bestandteile in Erzählungen. Insbesondere in künstlerischen Texten, in denen Ceordnetheiten in ihrer Wirkung stets sinnhaft und nicht zufällig erscheinen, ist die Klassifizierung der Wiederholungen entscheidend für die Charakteristik der Textstruktur. Rhythmische und grammatische Wiederholungen zählen dazu. Allgemeiner können bestimmte Figurenkonstellationen (Doppelgänger) ebenfalls als iterativ angesehen werden. «Sven Kosub: »Textuelle Berechenbarkeit«, in: Koschorke (Hg.): Komplexität und Einfachheit (2017), S. 240255, hier S. 251. Dies wiederum führt zu der überraschenden Idee, dass sich literarische Texte in gewissem Sinn als Programme auffassen lassen. Vgl. ebd., S. 240. Grundlage dieser Analogisierung ist der Prozesscharakter sowohl der Programmierung als auch der Erzählung. Schon Georg Lukács hat darauf für den Roman hingewiesen. Bei ihm jedoch wird der Prozess noch verstanden »als Wanderung des problematischen Individuums zu sich selbst [...] [von der, d.V.] sinnlosen Wirklichkeit zu klarer Selbsterkenntnis." Georg Lukács: Die Theorie des Romans. Ein geschichtsphilosophischer Versuch über die Formen der großen Epik [1920], 3. Aufl., Darmstadt et al.: Luchterhand 1976, S. 70. Diese Auffassung rückt ihn wiederum in die Nähe von Vargas Llosas Verständnis des totalen Romans. 
kommt und so ein Überschuss an Möglichkeiten, d.h. möglichem Verhalten gegenüber den aktuellen Erfordernissen, erzeugt wird.

Versucht man nun die beiden Modi von Emergenz, Rückkopplungsschleife und allgemeine Rekursion, den Wechselwirkungen innerhalb der zuvor besprochenen Romane genauer zuzuordnen, so fällt auf, dass ihr unterscheidendes Merkmalspaar Linearität/Nicht-Linearität unmittelbar mit deren je spezifischen textuellen Mustern korrespondiert. Während in Herr Gustafsson persönlich die Erzählung am Ende des Romans in Form einer seltsamen Schleife linear rückgekoppelt an dessen Beginn anschließt, ist das Erzählen, der Erzählprozess als Ebene der Signifikation, in den beiden anderen Narrationen durch nicht-lineare Rekursion in Echtzeit (jedenfalls tendenziell) mit ihren jeweiligen Bezugsebenen verkoppelt. Dies betrifft sowohl die Hereinnahme extradiegetischer Wirklichkeit in den Text, also all dessen, was dem Autor während des Schreibprozesses geschieht bzw. durch das Raster seines Wahrnehmungsapparats selektiert wird, als auch die Interaktion zwischen den virtuellen Realitäten und der Realwelt auf dem Niveau der Intradiegese. In jedem Fall handelt es sich um nicht-lineare, beständig stattfindende Austauschbeziehungen und nicht um eine einmalige Rückkopplungsschleife, bei der eine Verhaltensänderung (bzw. modifizierte Lesart) nicht unmittelbar, sondern erst nach Durchlaufen des gesamten Erzähl-Zyklus in streng linearem Modus eintreten kann. Die nicht-lineare Rekursion erweist sich somit als Komplexion der Rückkopplungsschleife im Sinn ihrer elementaren Funktion, d.h. als ihr verallgemeinerter operativer Modus in Bezug auf den Zusammenhang des Systemganzen literarischer Kommunikation. ${ }^{16}$ Daraus ergibt sich nun,

16 Iser geht sogar so weit, das Modell der generativen Matrix, von der auch ANH im Zusammenhang seiner Poetik-Vorlesungen sagt, dass sie den Verlauf des Schreibprozesses prognostisch bestimme ( $v g l$. Kap VI), durch die universellere Vorstellung allgemeiner Rekursion zu ersetzen. Damit ließe sich vermeiden, dass die durch (Re-)Kombination von Komponenten innerhalb einer Matrix entstehenden Resultate als Produkte verstanden werden können. Eine Vorstellung, die der Definition von Emergenz als ständiges Hervorbringen dynamischer Realitäten, die nicht zum Produkt gerinnen, zuwiderliefe. Vgl. ebd., S. 89. Die Matrix wäre, sofern sie mithilfe der Kombination unterschiedlicher Komponenten prognostische Aussagen darüber macht, was wird, in historischer Perspektive eine Spielart der Ars Combinatoria, wie sie aus dem späten Mittelalter etwa bei Ramón Llull (1232/35-1316) bekannt ist, in neuem Cewand. Bedenkt man die zum Selbsterhalt komplexerer Systeme notwendige »vielgliedrige Rekursion « (ebd., 89) in Echtzeit, erscheint Isers Vorschlag, die generative Matrix zugunsten allgemeiner, nicht-linearer Rekursion zu historisieren, er selbst spricht von ihr als »asylum ignorantiae [Herv. i.O.]«, (ebd.) weitgehend plausibel. Nur so lässt sich Emergenz in ih- 
was den Grad der Interaktivität zwischen den Elementen bzw. Bezugsebenen angeht, auf Basis der besprochenen Romantexte eine aufsteigende Folge hinsichtlich der Zunahme von explizit gemachter Komplexität. Während in Gustafssons Roman die Erfahrung von Kontingenz zu einer tiefgreifenden, existenziellen Sinnkrise führt, die mit sozialer Isolation verbunden ist und erst im Prozess der Versprachlichung und des Hervorbringens der Erzählung bewusst gemacht und weitgehend bewältigt werden kann, wird der Zufall im Fall ANH als systemische Randbedingung der Romanproduktion relevant und mutiert bei Kermani schließlich zum Lebenselixier des Schreibenden in der Auseinandersetzung mit der Wirklichkeit. Der Zufall verbunden mit der Vorstellung, dass alles auch anders sein könnte, als es ist, wird damit sogar zur Grundlage einer Poetik, die auf der tendenziell echtzeitlichen Austauschbeziehung zwischen Realität und Imagination aufruht. Er verliert damit, wie noch bei Gustafsson, an ursprünglicher Bedrohlichkeit, was er nun an produktiver Faszination freizusetzen vermag.

Versteht man des Weiteren Rekursion mit Iser im Sinne Varelas als »recursive histor[y] $\ll^{17}$ eines Systemganzen in Auseinandersetzung mit seiner Umwelt, so kann auf diese Weise verständlicher werden, wie der Zusammenhang von Schreiben und Leben bzw. die Möglichkeit verändernder Intervention literarischer Praxis auf das Leben ihrer Produzenten und Rezipienten unter Bedingungen von Echtzeit vorstellbar wäre. Schreiben/Lesen erscheint dann als Bewusstseinspraxis von Subjekten, die - analog zur Verhaltensmodulation von biologisch oder technisch realisierten Systemen unter veränderten Umweltbedingungen - bestrebt sind, durch ihre Aktivität im Prozess literarischer Signifikation Antworten auf ihnen in der Realität begegnenden Problemen zu generieren. Schreiben/Lesen ließe sich dann nicht nur als reine Bewusstseinspraxis ohne realweltlichen Bezug verstehen, sondern wäre zudem eine Art von Verhalten, um mit veränderten Bedingungen der Umwelt (politisch, gesellschaftlich, ökonomisch und technisch gesehen) zurecht zu kommen. Dass ein so verstandenes Schreiben/Lesen auf der Ebene der mentalen Bewältigung unvorhersehbar erfolgter, disruptiver Verwerfungen und damit zur Genese angemessener Verhaltensantworten durch die betroffenen Subjekte in relevantem Maße beitragen kann, ist auch einer der Ausgangspunkte des kreativen Schreibens (Creative writing). Gemeint ist damit in erster Linie

rer Qualität »als eine dynamische Realität kaleidoskopisch wechselnder Figurationen, die alles andere als ein Produkt sind« angemessen beschreiben. Ebd. 
die aus der kognitiven Psychologie stammende Einsicht, dass die mit dem kreativen Prozess einhergehende Identitätsstabilisation als Resultat der Elaboration schriftlicher Kommunikationsfähigkeit in der Auseinandersetzung des in (Re-)Formation befindlichen psychischen Subjekts mit seiner Umwelt begriffen wird. ${ }^{18}$

Wie verhält sich nun aber diese Möglichkeit der Entwicklung von Verhaltensvarianten, die auch im Rahmen der Adaptation eigenen Verhaltens an eine komplexer gewordene Umwelt (gemäß der empirischen Verstehensforschung) wirksam wäre, angesichts des von Baudrillard postulierten Verschwindens der Differenz zwischen der Realität und ihren medialen Repräsentationen? Wenn die in der Differenz zum Ausdruck kommende Dichotomie zugunsten eines einzigen, umfassenderen Prozesses subjektiver Wahrnehmung verschwände, wobei das Medium nur eine Schaltstelle innerhalb des rekursiven Zirkels der Wahrnehmung wäre, gäbe es prima facie in der Tat keine Möglichkeit der semiologisch-epistemischen Einschließung im Raum reiner Immanenz, dem Hier und Jetzt, zu entkommen. ${ }^{19}$ Alles, was innerhalb dieses großen Zirkels an systemkritischen Gedanken oder subversiven Ideen, an Abweichungen bzw. Alternativen zum gegebenen Status quo in Umlauf gerät und sich damit aus der hermetischen Innerlichkeit des Subjekts löst, wird in kurzer Zeit adaptiert oder verworfen und auf diese Weise konform gemacht. Damit erklärt sich zugleich die mittlerweile eingetretene Insuffizienz der Literatur, ein utopisches Gegenbild zur bestehenden Wirklichkeit zu entwerfen. Wenn aber mit dem Verschwinden der Differenz zwischen den beiden ontologisch verschiedenen Zuständen, der Realität und ihrer Repräsentation, sowie der daraus resultierenden tendenziellen Ununterscheidbarkeit von Realität und Fiktion die Möglichkeit der literarischen Utopie obsolet geworden ist, wie soll dann umgekehrt eine Veränderung des Blicks auf die bestehende Welt und des Lesers auf sich selbst, d.h. eine veränderte Interpretation des eigenen Wirklichkeitsausschnitts, des eigenen Fensters zum großen Ganzen durch komplexes Erzählen möglich sein?

18 Vgl. Lutz v. Werder: Lehrbuch des kreativen Schreibens, Wiesbaden: Marix 2007, S. 33.

19 Wie bereits im Zusammenhang der Blending-Theorie erläutert, handelt es sich um "generelle Wahrnehmungsprozesse [...], Prozesse globaler kognitiver und emotionaler, sogar körperlicher Wirklichkeitskonstruktion«, die auch bei der Beschäftigung mit Literatur bzw. Film eine Rolle spielen und nur auf der Grundlage eines vorgegebenen Medienangebots kaum erklärbar wären. B. Scheffer: Interpretation und Lebensroman, S. 24 . 
Als Domäne, in der noch nicht alle Inhalte anschlussfähig und wiederverwendbar gemacht sind, kommen dafür gestaltbildende Prozesse in Frage, wie sie bei der Produktion und Rezeption von avancierter Literatur und Kunst im Spiel sind. ${ }^{20}$ Dazu sind in jedem Fall Umstände erforderlich, die es den Subjekten erlauben, in kreativen Prozessen der Herstellung und Aneignung mit sich selbst und dem Werk in einen Möglichkeitsraum einzutreten, sodass ihre Identität nicht mehr als eine nur gleichförmig monotone, immer schon gegebene erlebt werden muss, sondern umgekehrt als eine in permanenter Formation befindliche begriffen werden kann. Auf diese Weise erscheint es möglich, der Schimäre endlos bestimmter Identität, deren Entwicklungsmöglichkeiten aufgrund von Systemzwängen still gestellt sind, durch Indetermination zu begegnen, die sich innerhalb kreativer Räume gegen das indifferente System der Überwachung und Kontrolle wenden lässt. ${ }^{21}$ 


\section{Zum Problem der Übertragbarkeit von Begriffen aus der Theorie komplexer Systeme in den literaturwissenschaftlichen Diskurs}

Wie Friedrich Cramer in seiner grundlegenden Monografie Chaos und Ordnung gezeigt hat, hängt der Grad von Komplexität eines Systems eng mit seiner Beschreibbarkeit zusammen. ${ }^{1}$ Je mehr Parameter für seine Beschreibung notwendig sind, umso komplexer ist es. Definiert man Komplexität (K), angelehnt an den Informationsbegriff, ${ }^{2}$ durch die Anzahl der Kombinationsmöglichkeiten, die ein System bei der Anordnung seiner Elemente hat, so lässt sich dies numerisch als Logarithmus der Zahl seiner möglichen Zustände (N) mit der Formel $\mathrm{K}=$ Log $\mathrm{N}$ ausdrücken. Bedenkt man die astronomisch hohe Zahl möglicher Kombinationen sprachlicher Elementareinheiten, aus denen ein literarischer Text hervorgehen kann, so kommt es in einem weiteren Schritt darauf an, welche der realisierbaren Zustände man in Betracht ziehen will. Dies ist notwendig, sobald man über die Ebene rein numerischer Deskription hinausgelangen und die Perspektive sprachlicher Beschreibung von Wirklichkeit (Weltaneignung) durch ein erzählerisches Subjekt (als Bezugssystem) berücksichtigen will. Es ist folglich der Prozess der Versprachlichung von Vorstellungen, also die Konfrontation der potenziellen Unendlichkeit sprachlicher Möglichkeiten mit dem, was der Erzähler sagen, an Wahrnehmungsbildern zum Ausdruck bringen will, der dem fertigen Text als abgeschlossenes Produkt vorangeht. Wenn Koschorke schreibt: »Erzählen ist eine

Vgl. Friedrich Cramer: Chaos und Ordnung. Die komplexe Struktur des Lebendigen, 2. Aufl., Stuttgart: Deutsche Verlags-Anstalt 1989, S. 275.

2 Dieser besagt, dass je komplexer ein System ist, desto mehr Information mit seiner Hilfe kodiert werden kann. 
hochgradig selektive Tätigkeit. Es hebt wenige Einzelzüge als signifikant aus einer Masse von Daten heraus «, ${ }^{3}$ so ist eben dieser Vorgang damit gemeint. Da dieser Prozess aber nicht beobachtbar ist, weil er in der operationell geschlossenen Sphäre subjektiver Kognition (Innerlichkeit) stattfindet, bleibt, abgesehen von den Selbstbeobachtungen der Autoren (einschlägig etwa in ANHs Poetikvorlesung über den Kybernetischen Realismus), neben der produktionsästhetischen Theorie nur die Beschäftigung mit vorliegenden Texten, die in den Bereich wissenschaftlicher Rezeption fällt. Dabei ist zu beachten, dass der Versprachlichungsvorgang nur das in die erzählerische Darstellung hereinlässt, was für den Fortgang der Handlung bedeutsam ist.

An dieser Stelle findet vorab also eine zweifache Reduktion statt. Zunächst einmal ist das menschliche Sensorium aufgrund seiner vielfältigen Modalitäten (visuell, auditiv, taktil, olfaktorisch sowie gustatorisch) und deren simultan erfahrbarer Phänomenologie komplexer, als die dafür zur Verfügung stehenden sprachlichen Ausdrücke. ${ }^{4}$ Dann ist zu berücksichtigen, dass nur ein Bruchteil des Geschehens der Wirklichkeit für den sich konkretisierenden Erzählvorgang in Frage kommt, die überwältigende Menge des potenziellen Gesamtgeschehens jedoch nur im Sinne einer Randbedingung den Vorgang begleitet und damit faktisch ausgeblendet wird. Was schließlich als manifester Text beim Leser ankommt, ruft in vielen Hinsichten unartikuliert bleibendes, aber implizit vorhandenes Weltwissen auf und korrespondiert mit Voreinstellungen, d.h. Erwartungen hinsichtlich bestimmter Erzählmuster, die aus der bisherigen Erfahrung geläufig sind, was sich wiederum komplexitätsmindernd auf die Rezeption auswirkt. ${ }^{5}$

Informationstheoretisch gilt zudem, dass ein System, wie Cramer sagt, dann "fundamental komplex" wird, wenn die algorithmische Beschreibung seiner Struktur "vergleichbar groß wie das System selbst wird. $\aleph^{6}$ Es ist dann nicht mehr programmierbar und seine Struktur wird, wie man sagt, indeterministisch, weil sie sich einer verlässlichen Prognose weitgehend entzieht. Die Grenzen der Beschreibbarkeit sind dann erreicht. Bleibt man bei dieser Definition, ließe sich für das komplexe Erzählen analog davon sprechen, dass fundamentale Komplexität dann gegeben ist, wenn die wissenschaftliche Beschreibung eines komplexen Erzähltextes umfangreicher wird als das litera- 
rische Werk selbst. Das Phänomen ist dann nicht mehr reduzierbar und die Sekundärliteratur überwuchert den Primärtext oft um ein Vielfaches, wie z.B. im Fall von Musils Mann ohne Eigenschaften. Umgekehrt führt dieses Verhältnis dazu, dass die Ansprüche an die Analysefähigkeit eines wissenschaftlichen Beobachters desto größer werden, je komplexer sich ein System gestaltet, das betrachtet werden soll. Es bedeutet zudem, anders als bei subkritischer Komplexität, ${ }^{7}$ dass der Beobachter in eine umfassendere Interaktion mit dem System eintritt, wobei nicht mehr von den kognitiven Prozessen der Wahrnehmung und des Denkens abstrahiert werden kann, die unhintergehbar mit seiner Person verbunden und für die komplexe Beobachtung essenziell sind. Die scharfe Trennung zwischen Subjekt und Objekt der Beobachtung wird somit hinfällig, weil das Objekt sich im Wahrnehmungsfeld der subjektiven Perspektive des Beobachters erst konstituiert und nicht schon vorher in einem absoluten Sinn gegeben ist. Es resultiert also ein Darstellungsproblem, das darin besteht $\mathrm{zu}$ verstehen, worum es sich bei dieser Interaktion überhaupt handelt und auf welche Weise sie angemessen beschreibbar wäre.

Dieser Effekt, der zum ersten Mal im Zusammenhang der Analyse von physikalischen Quantensystemen als Unschärferelation von Werner Heisenberg beschrieben wurde, ist bei der Analyse aller Systeme zu beachten, deren Verhalten sensibel für Einflussfaktoren aus der Umwelt ist, bis hin zur Konstitution des Systemobjekts durch die Wahrnehmung eines Beobachters bzw. deren Erweiterungen durch Messinstrumente oder optische Apparaturen. Hier wird deutlich, dass die verbindende Analogie zum System literarischer Kommunikation und seiner Partitionen, insbesondere der Interaktion zwischen Text und Leser, die hier hauptsächlich interessiert, im Paradigma der Erzeugung des Gegenstands durch Wahrnehmung besteht. Wenn der Text, wie erläutert, erst im Vorgang der Rezeption, also indem der Leser ihn wahrnimmt, Bedeutung erhält, so lässt sich dieser Umstand in systemtheoretischer Perspektive des Weiteren so beschreiben, dass der literarische Text als emergente Realität durch die Aktivität des Lesers als kulturellem Akteur in Auseinandersetzung mit seiner kulturellen Umwelt, dem Text, der vor seiner Rezeption nur potenziell existent ist, erst hervorgebracht wird.

Vergleicht man hochkomplexe Systeme, z.B. das Evolutionsgeschehen der biologischen Arten, die Populationsdynamik bei Insekten, die Strömungsdynamiken und Klima-Modelle der Meteorologie, das Mehrkörperproblem der

$7 \quad$ Zur Definition von subkritischer, kritischer und fundamentaler Komplexität vgl. ebd., S. $279-286$. 
Himmelmechanik oder das Konnektom des menschlichen Gehirns mit dem System literarischer Kommunikation, so wird der Komplexitätsgrad, was komplexes Erzählen betrifft, innerhalb der Partitionen (Produktion, textuelle Struktur, Rezeption) zumeist im Bereich kritischer Komplexität liegen. Dies bedeutet, worauf schon Cramer im allgemeinen Kontext hingewiesen hat, dass der Prognostizierbarkeit des Verlaufs innerhalb dieser Teilbereiche/Subsysteme zwar praktische aber keineswegs grundsätzliche Grenzen gesetzt sind. ${ }^{8}$ Will man allerdings den Gesamtprozess berücksichtigen, so reicht dieser, ausgehend von den Anfangsbedingungen, d.h. der Autorintention, dem $\mathrm{zu}$ behandelnden literarischen Stoff, den $\mathrm{zu}$ beachtenden Gattungskonventionen und den Erwartungen aufseiten von Literaturbetrieb bzw. Leserschaft bis hin zur Distribution und Rezeption des >fertigen aufgrund der Vielzahl der miteinander interagierenden Komponenten und der daraus resultierenden, emergenten Dynamik in den Bereich fundamentaler Komplexität hinein. Dies hat zur Konsequenz, dass trotz weitgehend determinierter Ausgangsbedingungen keine Möglichkeit einer verlässlichen Prognose über den Systemverlauf insgesamt besteht. Vom Initial mit den genannten Komponenten bis zu den möglichen Lesarten in der Rezeption spielen so viele heterogene Faktoren in den Systemprozess hinein, der aus diesem Grund auch nicht als Kontinuum, sondern nur als multifaktorielles, diskontinuierlich verkettetes Geschehen konzipierbar ist, dass sich unmöglich sagen lässt, was schließlich beim Leser ankommen wird. Es ist, was die Aufnahme des Textes angeht, buchstäblich alles möglich. Von weitgehend konformen Lesarten - gemessen an den Erwartungen von Autor und Verlag - bis hin zu unvorhersehbar überraschenden Interpretationen im positiven wie negativen Sinn. ${ }^{9}$

Ein kritischer Einwand, der gegen die Übertragung von Paradigmen aus der Komplexitätstheorie auf das System literarischer Kommunikation erhoben werden könnte, besteht darin, dass es hier keine diskreten Daten in Form von Messergebnissen o.Ä. gibt, sondern in der Hauptsache kreative Prozesse bzw. inferenzielle Operationen des schreibenden, lesenden und deutenden

\footnotetext{
8 Vgl. ebd., S. 280.

9 Dieser Effekt wird sich bei einer nicht-intentionalen Werkgenese noch verstärken, da hier der Zufall als Element des Unvorhersehbaren zum Bestandteil des manifesten Textes wird. Dies wiederum wirkt sich erfahrungsgemäß hemmend auf die Rezeption aus, da nur ein zahlenmäßig geringes Publikum von Spezialisten mit entsprechenden Vorkenntnissen in der Lage ist, solche Texte gewinnbringend zu lesen.
} 
Beobachters, die als solche nicht äußerlich beobachtbar sind. Da aber diese Prozesse im kognitiven System des Produzenten/Rezipienten stattfinden, ist davon auszugehen, dass die betreffenden Formen von Interaktivität (kognitiv, emotional, evaluativ, ästhetisch) in das Feld fundamentaler Komplexität hineinreichen, das mit der Aktivität des menschlichen Gehirns verbunden ist, gleichgültig ob diese Prozesse nun direkt beobachtbar sind oder nicht. Dem Vorwurf, dass Begriffe und Methoden aus der Komplexitätsforschung für den im Vergleich dazu heterogenen literaturwissenschaftlichen Diskurs unangemessen seien, lässt sich zudem verhältnismäßig leicht durch einen Hinweis auf den hohen Grad an Allgemeinheit entkräften, den die Theorie mittlerweile erreicht hat. ${ }^{10}$ Sie ist deswegen auf viele Systeme außerhalb der Naturwissenschaft anwendbar, z.B. die Geschichtsschreibung (unterschiedliche Geschichtssysteme), die mathematische Modellierung und digitale Simulation von Finanzmärkten oder gesellschaftlichen Soziodynamiken und eben auch auf ästhetische Phänomene, wie die Strukturanalysen der zuvor behandelten literarischen Texte gezeigt haben. Dies hat seinen Grund vor allem darin, dass die Eigenschaft fundamentaler Komplexität, tritt sie einmal auf, nicht kompensiert werden kann. ${ }^{11}$ Selbst dann nicht, wenn mithilfe von immer leistungsfähigeren, technischen Ressourcen wie Teilchenbeschleunigern und Hochleistungsrechnern eine Realität - in diesem Fall die Welt der Quarks - sichtbar gemacht werden soll, die anders nicht erkennbar wäre. ${ }^{12}$

10 Vgl. dazu den Abschnitt »Komplexität und Philosophie« in: Mainzer, Komplexität (2008), S. 108-117, hier S. 115-117, der eine kurze Zusammenfassung der ethischen Konsequenzen der Komplexitätsforschung bietet. Vgl. außerdem F. Cramer: Chaos und Ordnung, S. 282-284.

11 Vgl. ebd., S. 281.

12 Es zeigt sich wiederum, dass bei der Auseinandersetzung mit steigender Komplexität aufseiten des Phänomens die Theorie zu ihrer Bewältigung ihrerseits komplexer wird. Dies gilt gerade dann, wenn beteiligte Teiltheorien nicht aufeinander reduzierbar sind, in diesem Fall die Quantenphysik und die Allgemeine Relativitätstheorie. Bisher ist die Beschreibung sehr großer Massen und Beschleunigungen in der Relativitätstheorie nicht kompatibel mit der Quantentheorie der Wechselwirkungen kleinster Teilchen, weil sich die in der makroskopischen Gravitation wirkenden Kräfte nicht in Elementarportionen, den Quanten, einteilen lassen. Die dafür notwendige Theorie einer Quantengravitation steht bisher noch aus. Als aussichtsreichster Kandidat gilt die String-Theorie, deren Lösung nach bisherigem Kenntnisstand jedoch nur in einem Universum mit bis zu 26 Dimensionen möglich scheint. Vgl. dazu https://de.wikipedia.org/ wiki/Quantengravitation vom 06.08.2020. 
Gebraucht man den Begriff Komplexität hingegen pauschal, kommt man nur zu ganz oberflächlichen, redundanten Aussagen, weil Komplexität neben der allgemeinen Relevanz in jedem Bereich kontextspezifische Bedeutungen hat, die nicht in andere Zusammenhänge übertragbar sind. Dies ist auch der Grund dafür, warum die These eines globalen Zusammenhangs der Dinge, von der anfangs die Rede war, kaum über die plakative Formel, dass alles mit allem zusammenhängt, hinausreicht, wenn man sie so stehen lässt. Neben ihrer Kontextsensitivität ist es zudem für komplexe Phänomene typisch, dass sie nur durch teilnehmende Beobachtung beschreibbar sind. ${ }^{13}$ Dass man als unbeteiligter Beobachter nicht an sie herankommt, wird, wie bereits an der Analogie zur Unschärferelation erläutert, an einem wohl noch naheliegenderen Beispiel für fundamentale Komplexität erkennbar. So lässt sich mit Cramer in Hinsicht auf das »Netzwerksystem Leben ${ }^{14}$ sagen, dass jeder Lebende nicht nur ständig von ihm umgeben, sondern auch selbst eines seiner Elemente ist, sofern er mit anderen Organismen bzw. seiner Umwelt interagiert. Daraus resultiert, dass es nicht möglich ist, ein hypothetisches Ganzes, von dem sich herausstellt, dass die analysierende Instanz selbst nur eine seiner Positionen ist, gewissermaßen von außen aus der Perspektive eines unbeteiligten Beobachters als integrales Gesamtgeschehen beschreiben zu wollen. Analog verhält es sich im Fall der Konstitution des Erkenntnisobjekts im Prozess der Wahrnehmung des beobachtenden/rezipierenden Subjekts, sei es nun ein Quantensystem oder ein literarischer Text, die analysiert werden sollen, da auch hier nicht eindeutig entscheidbar ist, welche Anteile der Beschreibung dem Subjekt und welche dem beobachteten System zukommen. Um diese Unterscheidung treffen zu können, müsste das Subjekt aus sich heraustreten und somit von sich in einer Weise abstrahieren können, wie es nur in der literarischen oder filmischen Narration möglich ist, nicht aber in der empirischen Realität des Humanums.

Wie Cramer richtig bemerkt hat, bliebe innerhalb einer solchen epistemischen Konstellation »immer eine Bestimmungsgröße zu wenig «, ${ }^{15}$ um die gesamte Netzwerkstruktur zu erfassen. Diese fundamentale Unbestimmbarkeit resultiert eben daraus, dass das erkennende Subjekt erstens immer nur einen Ausschnitt dieser Gesamtrealität zu erfassen vermag, die sich an den

\footnotetext{
13 Teilnehmende Beobachtung wird hier nicht als Methode der Sozialwissenschaften verstanden, sondern als erkenntnistheoretische Kategorie.

14 F. Cramer: Chaos und Ordnung, S. 281.

15 Ebd.
} 
Rändern potenziell infinit fortsetzt und zweitens, damit zusammenhängend, in dieses System als Position eingebettet ist und sich deswegen nicht im Sinne objektiver Erkenntnis darüber erheben kann. Objektiv erkennbar gemäß diesem Verständnis wären nur Artefakte (z.B. Präparate) bzw. Prozesse innerhalb limitierter, experimenteller Anordnungen, nicht aber der umfassende Prozess des Lebendigen, aus dem diese Erkenntnisobjekte ja gerade herausgelöst und für die wissenschaftliche Betrachtung sozusagen sstillgestellt< sind. Radikal gesprochen gewinnt eine solche Erkenntnisweise ihre Ergebnisse um den Preis des Lebens selbst. ${ }^{16}$ 



\section{Das medial in sich eingeschlossene Subjekt - Zum Verhältnis von Literatur und Leben unter Bedingungen der Echtzeit}

Im gegebenen Zusammenhang liegt es nahe, die vorangegangenen Überlegungen mit einer Reflexion des Verhältnisses von Literatur und Leben fortzusetzen. Ein Problem, das bereits von den historischen Avantgarden gesehen wurde, die angesichts dessen, was Peter Bürger in seiner Theorie der Avantgarde als "Erfahrungsschwund ${ }^{1}$ in der arbeitsteilig gewordenen, bürgerlichen Gesellschaft bezeichnet hat, die Rückführung von Kunst in Lebenspraxis fordern. ${ }^{2}$ Soll damit der gesamtgesellschaftlichen Folgenlosigkeit künstlerischer Produktion angesichts ihrer Segregation aus lebenspraktischen Zusammenhängen in die autonome Sphäre ästhetischer Erfahrung begegnet werden, so fragt sich, ob unter Bedingungen der Postmoderne das Problem der Trennung beider Bereiche fortbesteht oder durch die Entwicklung der Medien und der digitalen Vernetzung der Gesellschaft, d.h. der damit in Aussicht gestellten schrankenlosen Kommunikation, verschwindet.

So ist zu konstatieren, dass die Arbeitsteiligkeit im Vergleich zur Epoche der historischen Avantgarden sich bis in die Gegenwart immer weiter ausdifferenziert hat. Die im Vergleich höhere Spezifizität sowie Dynamik (und damit Komplexität) des gegenwärtigen Arbeitsmarktes ist dafür ein sicheres Indiz. In kurzer Zeit entstehen neue Berufsbilder, ja ganze Berufszweige, beispielsweise die IT-Branche, und verschwinden traditionelle, etwa in der Kohleindustrie. Der damit verbundene Erfahrungsschwund - auch gesamtgesellschaftlich gesehen - und die zunehmende Sprachlosigkeit zwischen den

1 Peter Bürger: Theorie der Avantgarde, Frankfurt a.M.: Suhrkamp 1974, S. 43.

2 Vgl. ebd., S. 29. 
Mitgliedern einer hochspezialisierten Berufs- und Bildungselite sowie der übrigen Gesellschaft, besonders dem Prekariat, dürfte sich im Vergleich nicht wesentlich geändert haben, wenngleich die Gründe dafür sicherlich andere sind. Im Zuge der politischen Demokratisierung der Gesellschaft im Verlauf der zweiten Hälfte des 20. und der digitalen Mediatisierung seit Beginn des 21. Jahrhunderts stehen die meisten Sachinhalte, deren Kenntnis für die Partizipation an den genannten Eliten unumgänglich ist, allen Mediennutzern in öffentlichen Bibliotheken, auf dem freien Markt oder im Internet zur Verfügung. All dies ist frei zugänglich und das Angebot ist sogar unübersehbar vielfältig geworden. Dies gilt in gleicher Weise für literarische Texte und den dazugehörigen wissenschaftlichen Apparat.

Die Inhalte treffen dabei jedoch auf ein Subjekt, das, wie Cramer schreibt, »überwiegend das bleiben [muss], was [es] geschichtlich schon war «. ${ }^{3}$ Damit ist gemeint, dass aufgrund der Selbstbezüglichkeit menschlichen Denkens und Handelns, ${ }^{4}$ neben der aus logischen Gründen ausgeschlossenen, totalen Explikation dieser Fähigkeiten/Eigenschaften, ${ }^{5}$ eine Adaption an veränderte Bedingungen der kulturellen (und natürlichen) Umwelt nicht in beliebigem Umfang möglich ist. ${ }^{6}$ Angesichts der rein funktionalen Betrachtungsweise, die dem Denken alles zutraut, erscheint es hier angemessener, die cartesische Vorstellung des vermittlungsgebundenen Subjekts, wie Hubert Dreyfus und Charles Taylor vorschlagen, zu dekonstruieren und durch ein Konzept verkörperten Verstehens zu ersetzen, bei dem anstelle des Subjekts, das sich beim Denken auf seine mentalen (inneren) Repräsentationen bezieht, ein »verkörperte[r] Akteur[...]« tritt, der »als engagiertes - eingebundenes - Wesen begriffen wird, als jemand, der in eine Kultur, eine Lebensform, eine >Welt $<$ des

3 F. Cramer: Chaos und Ordnung, S. 296.

4 Die Analogie zur Modulation des Verhaltens von biologisch bzw. technisch realisierten Systemen wird im Folgenden zugunsten der Perspektive selbstbewussten, intentionalen Handelns ersetzt (bzw. ergänzt). Deswegen wird im Unterschied zu den vorangegangenen Überlegungen aufgrund des ontologisch differenten Standpunkts der Betrachtung wieder vom Handeln und weniger von Verhalten die Rede sein.

5 Dies lässt sich etwa am Beispiel der mathematischen Logik zeigen, die nach Kurt Cödel unentscheidbare Aussagen enthält, welche nicht selbst wieder beweisbar sind, weil das axiomatische System, in dem sie Bedeutung erhalten, auf sich selbst rückgekoppelt, also selbstbezüglich ist. Vgl. F. Cramer: Chaos und Ordnung, S. 294 f.

6 Vgl. dazu ebd., S. 296 f. 
$\mathrm{Zu}$-tun-Habens eingebettet ist ". $^{7}$ Beide Faktoren, die an eine Herkunft gebundene Geschichtlichkeit und die Einbettung in eine durch Gewöhnung und Routine gekennzeichnete, im Akt der Wahrnehmung konstituierte, alltägliche Umwelt, führen dazu, dass die mit disruptiven Innovationen wie Computer und Internet zusätzlich entstehende Komplexität nicht in willkürlichem Maß durch Änderungen von Denken und Handeln der betroffenen Subjekte kompensiert werden kann. Angesichts dieser Limitationen zeigt sich, dass ein Verständnis des Subjekts als weitgehend abstrakter Schaltstelle oder Funktionseinheit (im Sinne Descartes) innerhalb des Paradigmas schrankenloser Interkonnektion zwischen Computer und humanem Nutzer rein praktisch schnell an seine Grenzen stößt.

Als informatischer Endverbraucher bzw. »Terminal-Bürger[...] «, ${ }^{8}$ wie Virilio sagt, sieht es sich unvermittelt und differenzlos (Baudrillard) mit Inhalten konfrontiert, die vor allem dann Interesse zu wecken vermögen, wenn sie mit seiner Vorstellung schneller Bedürfnisbefriedigung korrespondieren. Sobald es jedoch um intellektuell anspruchsvollere, ästhetische Inhalte und

Hubert Dreyfus/Charles Taylor: Die Wiedergewinnung des Realismus, übers. aus d. Engl. von Joachim Schulte, Frankfurt a.M.: Suhrkamp 2016, S. 171. Anstelle einer »inneren « Repräsentation der Wirklichkeit, die mit der räumlichen Vorstellung einer »inneren Welt« einhergeht, die von der äußeren Wirklichkeit abgegrenzt ist und damit ontologisch verschieden wäre, tritt bei Dreyfus und Taylor die Idee, dass es so etwas wie ein »Kontaktbild« (ebd., S. 174) mit einer dazugehörigen Kontakttheorie der Wirklichkeit (vgl. ebd., 4. Kap.) gibt, »wonach Erkenntnis darin besteht, daß wir mit der erkannten Wirklichkeit unmittelbar in Kontakt treten. «Ebd., S. 38. Dies bezeichnen sie als ursprüngliche Form des Umgangs mit Realität, bevor das reflektierende, sich schließlich auf sich selbst beziehende Denken in Form von Abbildung bzw. Kategorie verzögernd dazwischentritt. Der eigentliche Ort von Erkenntnis bestünde demnach in der Interaktion, dem Dazwischen der Begegnung von erkennendem Subjekt und zu erkennender Wirklichkeit und nicht in einem Jenseits desengagierter, reflexiv-kontemplativer Vernunft irgendwo im Inneren des Subjekts. Folgt man dieser Überlegung, so ließen sich auf diese Weise das Paradox der verschwundenen Welt sowie das HomunkulusProblem, die wesentlich aus dem Paradigma der Vermittlungsgebundenheit des Denkens und Vorstellens resultieren und deswegen in der Neurophilosophie eine wichtige Rolle spielen, vermeiden. Der Versuch eines neuen Framings der beiden Antinomien wird im Kontext fraktalen Erzählens in Kap. XVIII der vorliegenden Arbeit unternommen. Die detaillierte Explikation müsste jedoch aus Gründen der Diskurssystematik an anderem Ort erfolgen. Zu beiden Antinomien vgl. Cerhard Roth: Das Gehirn und seine Wirklichkeit. Kognitive Neurobiologie und ihre philosophischen Konsequenzen, Frankfurt a.M.: Suhrkamp 1997, S. 21 f. 
deren kreative Rezeption geht, kommt es nicht zu zielführenden, intellektuellen Transaktionen, sobald der motivierende Kontext fehlt, also die belebende Begegnung mit Gleichgesinnten oder Personen, die Kenntnisse und Knowhow weitergeben können und deren Beispiel zu eigenen Aktivitäten ermutigt. Obwohl sich im Vergleich die Angebote ja sogar vervielfältigt haben und auch die Verstärker-Relais wie Bücherzeiten in Radio und Fernsehen, Literaturkritik online, Studiengänge an Universitäten oder Unterrichtseinheiten an höheren, allgemeinbildenden Schulen für einen hohen Durchsatz an literarischer Information sorgen, führt das, solange der Nutzer nur Bestätigung für eigene Annahmen und Erwartungen im Netz sucht, nicht zwangsläufig zu mehr Erfahrung, sondern in erster Linie nur zu mehr Konsum. Es resultiert daraus zudem ein neues Problem, das der Entscheidungsfindung.

Wird dieses Problem rein kognitiv-zerebral angegangen, kann der Aspekt des körperlichen Zurechtkommens in der Welt, von dem auch Dreyfus und Taylor sprechen, ${ }^{9}$ nicht angemessen berücksichtigt werden. Im gegebenen Zusammenhang führt die Zunahme von Komplexität, solange sie nicht bewältigt werden kann, zu Orientierungslosigkeit und in der Folge zu Indifferenz, ohne dass daraus ein Handeln im eigentlichen Sinn resultiert. Es entwickelt sich zwar eine Eigendynamik, die jedoch zumeist schon im Vorfeld der genannten sekundären Erscheinungen stehen bleibt. So kommt es nicht zu einer Begegnung mit dem eigentlichen Phänomen, der Literatur, und die kommunizierten Erfahrungen sind nicht die eigenen, sondern sekundär adaptierte. Der angestoßene Prozess besteht phänomenologisch darin, dass redundante Gedankenschleifen solange aneinandergereiht werden, bis es zu einer Unterbrechung des Vorgangs von außen kommt.

Wenn, wie im Fall des komplexen Erzählens, dem literarischen Text die Fähigkeit zugestanden wird, Lesern neue Möglichkeiten des motivierenden Verstehens und des Erfahrungmachens zu eröffnen, so fragt sich hier erneut, wie dessen provokatives Potenzial, von dem auch Mieth spricht, ${ }^{10}$ angesichts eines medial (in sich selbst) eingeschlossenen Subjekts seine Wirkung entfalten soll. Denn neben dieser von Baudrillard und Virilio formulierten These des Einschlusses, der »Kokonisierung ", ${ }^{11}$ wie Virilio sagt, kommt nun sogar eine zweite Weise des Gefangenseins hinzu, die mit dem Scheitern der 
vermittlungsgebundenen Auffassung des Erkennens zusammenhängt. ${ }^{12}$ Weil die fundierungstheoretischen Annahmen der Wahrheitsbegründung, die auf Descartes zurückgehen, sich als inkohärent erweisen, resultiert mit Dreyfus und Taylor im Effekt ein vergleichbares Bild des »ohne Kontakt zu einer transzendenten Welt ${ }^{13}$ in sich gefangenen Subjekts. Um sich von dieser Vorstellung und den damit verbundenen Zuständen der Privation zu befreien, wird vorgeschlagen, den Bildcharakter der Wirklichkeit (Repräsentation) fallen zu lassen und durch ein Verständnis des fortwährenden Austauschs mit ihr zu ersetzen, das ohnehin das ursprünglichere wäre. ${ }^{14}$ Reduktionistisch ist an der ersten Vorstellung, dass es sich bei den mentalen, inneren Repräsentationen weitgehend nur um Zustände unseres Gehirns handelt. ${ }^{15}$ Die zweite geht hingegen von einer komplexeren, kontinuierlichen Interaktion aus, die mit der

Zum Motiv der Gefangenschaft durch das vermittlungsgebundene Bild vgl. Dreyfus/Taylor: Wiedergewinnung des Realismus, S. 113 und 125. Die Ausgangsposition der vermittlungsgebundenen Auffassung (Fundierungsgedanke) beschreiben Dreyfus und Taylor folgendermaßen: »In unserer Kultur ist ein gewaltiger Irrtum wirksam: ein effektives (Miß-)Verständnis dessen, was Erkenntnis eigentlich heißt, das auf vielen Gebieten unheilvolle Auswirkungen auf Theorie und Praxis nach sich gezogen hat. Um es in einer prägnanten Formel zusammenzufassen, könnte man sagen, daß wir das Erkennen als etwas Vermittlungsgebundenes (miß)verstehen. In ihrer ursprünglichen Cestalt ist diese Auffassung in dem Gedanken zum Vorschein gekommen, daß wir die äußere Wirklichkeit durch innere Vorstellungen oder Repräsentationen erfassen. So schreibt Descartes in einem Brief: >lch bin sicher, daß ich von dem, was außerhalb meiner selbst ist, keine Erkenntnis haben kann außer durch Vermittlung der Ideen in meinem Inneren. ¿ Dieser Satz erhält seinen Sinn vor dem Hintergrund einer bestimmten Topologie von Ceist und Welt. Die Wirklichkeit, die ich erkennen will, befindet sich außerhalb des Ceistes; meine Erkenntnis dieser Realität ist im Inneren. Diese Erkenntnis besteht in geistigen Zuständen, die den Anspruch erheben, genau das, was draußen ist, darzustellen. Erkenntnis findet dann statt, wenn diese Zustände die Realität tatsächlich in richtiger und zuverlässiger Form wiedergeben. Zur Erkenntnis der Dinge gelange ich nur durch die Vermittlungsleistung (»par l'entremise«) dieser inneren Zustände, die wir als >Ideen < bezeichnen können.«Ebd., S. 12 f.

14 Vgl. ebd., S. 135. Dreyfus und Taylor folgen in ihrer Kritik der vermittlungsgebundenen Auffassung dem Ansatz Wittgensteins, der in den Philosophischen Untersuchungen (§ 115) davon spricht, dass Wirklichkeit durch Sprache abgebildet wird und dieses Bild wiederum das Denken gefangen hält. Allerdings gehen die Autoren davon aus, dass andersherum das Bild sich in unserer Denk- und Lebensweise manifestiert und deshalb-gewissermaßen sekundär - in unserer Sprache zum Ausdruck kommt. Vgl. ebd., S. $11 \mathrm{f}$. 
Idee des Eingebettet-Seins in einer zuhandenen, unmittelbaren Umgebung und des engagierten Zurechtkommens in ihr korrespondiert. Mein Verständnis der Welt wird somit nicht nur von mir selbst konstruiert, sondern liegt in der Interaktion, dem Zwischenraum des Umgangs mit den Dingen. ${ }^{16}$

Dies bedeutet zudem, dass sowohl die Vorstellung autonomer Selbstgenügsamkeit des literarischen Textes als auch die autonomer Subjektivität hin zu einer stärker an Interaktion und Prozess orientierten, nicht essenzialistischen Konzeption beider Positionen revidiert werden müsste. Dabei muss Autonomie nicht gänzlich nivelliert werden, sondern kann als Verfasstheit von Positionen (hier Text bzw. Subjekt) innerhalb eines umfassenderen Netzwerks kommunikativer Strukturen erhalten bleiben. So kann berücksichtigt werden, dass ästhetische Produktion und Rezeption eigenen Gesetzmäßigkeiten folgen, die sich als Poetiken und Lesarten/Interpretationen nicht ohne Verlust auf allgemeinere, im Verhältnis zu ihnen unterkomplexe Prinzipien reduzieren lassen. Je nach Perspektive kann die komplexe Erzählung dann als kommunikatives, gesellschaftliches Handeln (wie in der ETL) oder als ästhetisches Phänomen verstanden werden, das sich (wie in der Erzählforschung) zuerst an Motiven und Strukturen des Erzählens orientiert. Auch hier wieder ist die eine Sichtweise nicht auf die andere reduzierbar. Vielmehr gehen beide von einem Phänomen aus, das sie in verschiedenen Hinsichten beobachten. Sie folgen dabei durchaus unterschiedlichen Logiken, was zur Folge hat, dass ein Transfer von Information/Wissen zwischen beiden Erkenntnisdomänen nicht ohne weiteres, wenn überhaupt, möglich ist.

Deshalb ist es mit Nassehi erforderlich, Strategien der Übersetzung zu finden, die es erlauben, Forschungsresultate des einen Bereichs für den anderen rekombinierbar zu machen, ${ }^{17}$ d.h., wie im vorliegenden Text mit Ergebnissen aus der Neurowissenschaft, der kognitiven Literaturwissenschaft, der Subjektphilosophie u.a. erfolgt, adaptiv zu >überschreiben<. Dazu gehört »ein Management von Unterbrechungen ${ }^{18}{ }^{8}$ wie Nassehi sagt, weil die einfache Übertragung von Bedeutungen vom einen in den anderen Bereich ohne entsprechende Restrukturierung aus diskurslogischen Gründen ausgeschlossen ist. So versteht sich auch seine These, dass wir nur deshalb kommunizieren müssen, weil wir uns unter komplexer gewordenen Bedingungen nicht (mehr)

\footnotetext{
16 Vgl. ebd., S. 175.

17 Vgl. A. Nassehi: Letzte Stunde der Wahrheit, S. 198 f. sowie Kap. II der vorliegenden Arbeit.

18 Ebd., S. 199.
} 
unmittelbar erreichen können. ${ }^{19}$ Dies gilt für die Teilnehmer verschiedener Diskurse bei dem Versuch, sich zu verständigen, ebenso wie für die Selbstkommunikation des Subjekts. Das jedenfalls dürfte bei der Erörterung von Herbsts Versuch, am Ende des Romans Buenos Aires. Anderswelt zu sich selbst $\mathrm{zu}$ kommen, deutlich geworden sein.

Nimmt man die vorangegangenen Überlegungen des eingebetteten, unmittelbaren, engagierten Zurechtkommens mit der Wirklichkeit hinzu, dann kann Herbsts Flucht aus der skünstlichen`Welt Garrafff (W3) zurück in Deters Realwelt Buenos Aires (W1) nun sogar als Parodie der vermittlungsgebundenen Auffassung verstanden werden. Geht diese davon aus, dass es keinen unmittelbaren Zugang zur Wirklichkeit als solcher gibt, sondern nur zu einer mentalen Repräsentation von ihr im Bewusstsein des Subjekts, so ist es exakt diese Vorstellung, die im Roman zur Schimäre wird. Indem die virtuelle Praxis des Löschens und Kopierens im Rahmen der transitorischen Prozesshaftigkeit personaler Identität ontologisiert wird, gerät das, was sich in der Imagination des Ich-Erzählers Herbst ereignet und als wirklich erlebt wird der Sprung von der einen in die andere Identität/Erzählebene/Realität -, zum intradiegetisch realen Geschehen.

Nun ist jedoch mit der literarischen Erzählung, abgesehen von der Identität des empirischen Autors, die über dessen Intention mit dem Text verbunden ist, die eigene Erfahrung, das gelebte Leben, soweit es zurückliegt und bis in die Gegenwart hineinreicht, erzählerisch zu gestalten und dadurch zu bewältigen, vorderhand gar keine personale Identität gemeint. Zwar lassen sich auch auf der Ebene der Diegese Identitäten postulieren (davon geht etwa die Narratologie aus!), so die des erzählten Geschehens/der erzählten Geschichte, des Erzählers und der erzählten Figuren, doch sind diese fiktiv und verstehen sich im Sinne eines Angebots an den Leser, die in diesen Figurationen dargestellten Erfahrungen zu teilen. Im Akt des Lesens interagieren nun diese in den sprachlichen Bedeutungen des Textes verdichtet dargestellten Inhalte mit der Imagination der Rezipienten, sodass je nachdem, wie der Text im Einzelnen und im Ganzen verstanden wird, die unterschiedlichen Lesarten subjektiv stark voneinander abweichen können. Was dieser Vorgang in seiner ganzen Komplexität für den einzelnen Rezipienten bedeutet, hängt nun entscheidend von der Wirklichkeit ab (der Umwelt und den Beziehungen, in denen er lebt), die als Hintergrund und Projektionsfläche seiner imaginativen Fantasie ausschlaggebend dafür ist, ob die literarische Erzählung für de- 
ren Verständnis, ihre Veränderung oder Bewältigung relevant zu sein vermag. Dies kann jedoch im Einzelfall nur der empirische Leser selbst entscheiden.

Sofern Denken (Theorie) und Handeln (Praxis) interaktiv aufeinander bezogen und nicht im Sinn ontologisch differenter Bezugssysteme voneinander getrennt wären, ließe sich mit Dreyfus und Taylor auch die Lektüre um einen Handlungsaspekt erweitern. Der Vorgang wäre dann nicht nur beschränkt auf handlungsarme Zustände kontemplativer Rezeption, deren Schauplätze sich fernab in den Tiefen entrückter, innerlich-auratischer Subjektivität annehmen ließen, sondern verbunden mit der Frage nach dem eigenen Engagement in einer als veränderungs- resp. verbesserungswürdig erfahrenen, u.U. und zuerst auch >nur< privaten Wirklichkeit. Dazu müssten die Rezipienten sich jedoch als Lesende zunächst erst noch entdecken - in einem performativen Prozess, in dem sie sich als Beobachter/Leser ihrer selbst bewusst werden. Dieser Vorgang ließe sich in etwa so illustrieren, dass dabei Wahrnehmung $\mathrm{zu}$ einem Ereignis/Erlebnis wird, derart, dass das, was das Ich des Lesers jetzt und hier wahrnimmt (innerhalb wie außerhalb des Textes), etwas zu bedeuten habe, denn sonst wäre es, das Ich, gar nicht für sich da, sondern nur Teil einer umfassenderen Verkettung von Umständen und Situationen, die seiner nicht achten. Und nicht nur das. Sie müssten sich nicht nur selbst lesen können, also ihr Handeln und schließlich sogar ihr eigenes Verstehen verstehen, ${ }^{20}$ sondern sich darüber hinaus als Teil einer Simulation begreifen, d.h. als Element eines Modells von Wirklichkeit, das sie sich nicht selber ausgesucht haben, mit dem sie vielmehr konfrontiert sind aufgrund der besonderen historischen Situation, in der sie leben, ganz so wie der Erzähler in Bodo Morshäusers Narration Die Berliner Simulation (1983). Erst dann wären Baudrillards und Virilios Thesen hinsichtlich der Wirklichkeit im Zeitalter von Echtzeit und globaler Vernetzung mit der daran gebundenen, veränderten Rolle des Subjekts angemessen berücksichtigt.

20 Vgl. dazu den Aufsatz von Heinz von Foerster: »Verstehen verstehen«, in Ders., Wissen und Cewissen. Versuch einer Brücke, hrsg. von Siegfried J. Schmidt, übers. aus d. Amerik. von Wolfram Karl Köck, Frankfurt a.M.: Suhrkamp 1993, S. 282-289. 
Der Schwundzustand der Entfremdung, ${ }^{21}$ sich selbst nicht zu fühlen, d.h. sich nicht wahrzunehmen, könnte dabei einem Selbstverhältnis weichen, in dem die präsentische Zeit sich mit der Gegenwart des Subjekts anfüllt. In einer derart als ausgedehnt, untief und zuhanden erfahrbaren Gegenwart wäre es ihm möglich, alle Zeiten, in denen es gelebt hat, in einem bewussten Beisich-Sein zu versammeln, sodass daraus ein Gefühl für die komplexe Fülle des eigenen Lebens zu erwachsen vermag. Auch wenn dies noch kein veränderndes Handeln im starken Sinn wäre, sondern eher eine Spielart ästhetischer Anschauung des eigenen Lebens, möglicherweise verbunden mit Vorstellungen davon, wie etwas anders/besser zu machen, wie anders/besser zu leben wäre - Vorstellungen, die inferenziell durchaus mit Lektüre-Erfahrungen verbunden sein können. Handeln könnte dann erst erfolgen, wenn erkannt ist, dass aus dieser Befindlichkeit allein noch keine sreale Veränderung resultiert. Dabei wiederum spielt das Begehren eine Rolle, also die Frage danach, wer und was ich sein will und wie dies zu bewerkstelligen wäre, solange ich es noch nicht bin. ${ }^{22}$ Leser und Leserinnen könnten auf diese Weise zu Experten

21 Hier lassen sich noch weitere Überlegungen zum Verhältnis von Entfremdung und Zeiterleben anknüpfen, die mit dem Verschwinden zeitlicher Dimensionalität aus dem Bewusstsein des Terminal-Bürgers zusammenhängen. Die wichtigste Ursache dafür kann in den simultan ablaufenden Interaktionen zwischen Sender und Empfänger im Internet gesehen werden. Für diese Art von Konnektivität zählt nur die gegenwärtige Interaktion, der Datenaustausch per Mausklick zwischen IT-Adressen und den damit verknüpften Datenendgeräten. Der zeitliche Horizont der Gegenwart dehnt sich auf diese Weise für das Bewusstsein, das an ein solches Gerät gekoppelt ist, endlos aus. Die Möglichkeit des zeitlichen Differenzials, das über die Formen der Vergangenheit, Gegenwart und Zukunft sowie ihrer Invertierungen bis hin zum Futur II reicht, ist als Struktur des menschlichen Bewusstseins, das die damit verbundenen Erfahrungshorizonte auch leben will, auf der Ebene des maschinellen Informationsaustauschs gar nicht implementiert. Sobald das Bewusstsein sich auf diese Ebene herablässt, also auf die Ebene der Schaltkreise und der damit verbundenen Mechanismen, geht die Dimension zeitlicher Tiefe verloren. Es gibt nur den gegenwärtigen Moment, der von einem nachfolgenden, verstanden als instantane Zustandsänderung des Systems, abgelöst wird. Es folgt phänomenologisch betrachtet ein Vergessen auf das andere. Ein Prozess, der sich auch beschreiben ließe als auf technische Weise implementierte Demenz von IT-Systemen, deren Funktionsabläufe sich nach menschlichen Zeitbegriffen in permanenter Gegenwart endlos nach vorne stürzen oder - im Fall der Störung - unvorhergesehen abbrechen.

22 Das Motiv der Selbstbestimmung, das damit angesprochen wird, ist freilich grundlegend für das menschliche Leben überhaupt. Es ist nicht nur ein Aspekt der Rezeption literarischer Texte, sondern trifft in noch stärkerem Maß für die Produktion zu, sofern 
des eigenen Lebens werden und dadurch das Maß ihrer Selbstwirksamkeit ${ }^{23}$ steigern, das abhängig ist vom Grad ihrer Bewusstheit.

Anders als Baudrillard, der das Vermögen der Einbildungskraft nicht bzw. nur negativ im Sinn der Extermination des linguistischen Wertes in seine Überlegungen einbezieht, ${ }^{24}$ soll im Folgenden mit Bezug auf Markus Gabriel dafürgehalten werden, dass sich menschliches Leben nicht auf die »Anwe-

dabei wesentlich die Identität des schreibenden Subjekts im Spiel ist. Es erweist sich damit, dass die Einbildungskraft, wie Markus Gabriel schreibt, unverzichtbar für unsere Selbstbestimmung ist. Vgl. M. Gabriel: Fiktionen, S. 28. Diese Motivation ist zentral für Autorinnen und Autoren, deren Werk, wie im Fall Wolfgang Hilbig, durch den Kampf um die eigene Identität als Schriftsteller geprägt ist. Bei Hilbig geht es nicht nur real, sondern zugleich auch in seinen Texten um die Konversion vom Arbeiter zum Schriftsteller. Indem das erzählende Subjekt im Schreibprozess sich selbst beobachtet, tritt es in ein existenzielles Verhältnis zum eigenen Sein. So kann der Schreibakt auch als »Sichzusichverhalten« (nach Ernst Tugendhat) verstanden werden, »weil es dem Subjekt hier darum geht, wie und was es sein will, d.h. wie es sich verstehen will, nämlich als Schriftsteller, und diese Intention als Teil der praktischen Frage begreift, wie das zu bewerkstelligen wäre.« André Steiner: Das narrative Selbst - Studien zum Erzählwerk Wolfgang Hilbigs. Erzählungen 1979-1991, Romane 1989-2000, Frankfurt a.M. et al.: Peter Lang 2008, S. 55.

23 Der Begriff der Selbstwirksamkeit spielt in der Bewusstseinsforschung eine wichtige Rolle. Demnach bemisst sich der Crad des Bewusstseins nach dem Grad der Selbstwirksamkeit, mit dem ein System auf sich selbst kausal zurückwirken kann. Vgl. „Christoph Koch über die Fußspuren des Bewusstseins, die Vorgänge in einer Mühle und das Bewusstseinsmeter «, in: Eckholt, Kann sich das Bewusstsein bewusst sein? (2017), S. 179-197, hier S. 186. Entscheidend dabei ist, dass Vergangenheit, Gegenwart und Zukunft eines Systems in Form von internen Systemzuständen miteinander interagieren können müssen (Feedback). Cenau dies bedeutet es für ein System, unabhängig davon, ob es biologisch oder technisch realisiert ist, bewusst sein zu können. Den eigenen Zustand intentional oder kausal beeinflussen zu können, heißt über Selbstwirksamkeit zu verfügen, was wiederum nur möglich ist durch Bezugnahme auf erwünschte, in der Zukunft liegende Verfassungen bzw. durch Abgleich mit Zuständen aus der Systemvergangenheit (vgl. ebd.). Überhaupt ist die Möglichkeit, bewusste Erfahrung zu machen, identisch mit dem Vermögen zu kausaler bzw. intentionaler Selbstwirksamkeit (vgl. ebd.), was wiederum eine Form von Komplexität darstellt, sofern dazu komplexe Rückkopplungen zwischen den verschiedenen zeitlichen Ebenen notwendig sind.

24 Er spricht davon, »daß der Bereich des Poetischen [...] ein Prozeß der Vernichtung des Wertes ist« (]. Baudrillard: Der symbolische Tausch, S. 303), was eine Auflösung des Signifikanten bedeute und damit eine unwiderrufliche Zerstreuung fester Referenzialität zur Folge habe. Vgl. ebd., S. 306. 
senheit in sensorischen Reizszenen ${ }^{25}$ reduzieren lässt, sondern weit darüber hinausreicht. Auf diese Weise besteht Hoffnung, einen Ausweg aus der doppelten Gefangenschaft zu weisen, von der zuvor die Rede war. So liegt für den mit Einbildungskraft Begabten das besondere Vermögen von Fiktionen darin, das Klaustrum des sensorischen Hier und Jetzt in Akten transzendentaler Vorstellungsbildung zu überschreiten. Gemeint ist damit eine »Transzendenz über das Gegebene ${ }^{26}$ hinaus, das uns jeweils im Modus unmittelbarer Anschauung gegenwärtig ist. In diesem Zusammenhang macht uns Gabriel mit Stanley Cavell darauf aufmerksam, dass Fantasie nicht als eine von der Wirklichkeit getrennte Welt zu verstehen sei. Dies sei nur eine reduzierte Auffassung, die mit ihrer eigentlichen Bedeutung kaum noch etwas zu tun habe. ${ }^{27}$ Ihr wichtigstes Vermögen besteht umgekehrt vielmehr darin, dass sie uns den Kontakt zur Welt erst ermöglicht, anstatt uns von ihr zu entrücken, indem sie die Zwischenräume der Bezugnahme auf Gegenstände ausfüllt und damit die Szenen unseres Wahrnehmungsfelds zusammenhält, sodass wir unsere Wahrnehmungsepisoden als stabiles mentales Kontinuum erleben können. ${ }^{28}$ Demnach konstituiert sich Wirklichkeit nicht nur in epistemologischen Bezugnahmen interaktiver Erkenntnis zwischen Subjekt und Umwelt (vgl. Dreyfus' und Taylors Ansatz), sondern besteht wesentlich in der Fähigkeit der Einbildungskraft (Imagination) über das sensorisch unmittelbar Vorhandene hinauszugehen und uns auf diese Weise - über den Zugang zu fiktionalen Gegenständen - mit einer umfassenderen Vorstellung von Wirklichkeit zu verbinden. ${ }^{29}$ So erst kann die Vorstellung einer Welt als ganzer, als "Gesamtlage ${ }^{30}{ }^{30}$ entstehen, in der wir uns als Menschen situiert finden. Im Sinne fiktionaler Gegenständlichkeit ergänzt werden muss also das, was wir nicht unmittelbar wahrnehmen bzw. nicht mehr erinnern können, sodass, wie schon Scheffer gesagt hat, unsere »endlos autobiographische Tätigkeit der Wahrnehmung « ${ }^{31}$ fortgesetzt werden kann.

Kommt es zu einer Kultivierung des inneren Blicks, der anhand von Lektüre sich fortbildenden Einbildungskraft, so ist anzunehmen, dass sich dieses

25 M. Gabriel: Fiktionen, S. 24.

26 Ebd., S. 25.

27 Vgl. ebd.

28 Vgl. ebd., S. $24 \mathrm{f}$.

29 Vgl. ebd., S. 27.

30 Ebd.

31 B. Scheffer: Interpretation und Lebensroman, S. 10, 23, 24 und passim. 
Vermögen, wo es die Möglichkeit hat, sich rein, also ohne störende Ablenkung durch die umgebende Wirklichkeit zu entfalten, bis hin zur Fähigkeit immer komplexerer Visionen entwickelt, ohne dass vorderhand $\mathrm{zu}$ sagen wäre, $\mathrm{zu}$ welchem (weiteren) Zweck eigentlich. Ist die Einbildungskraft so von ihrer Funktion empirischer Weltbildung zeitweilig entbunden, kann sie, wie etwa bei Lommel, dazu dienen, das eigene Leben in Richtung von Möglichkeiten zu erweitern, die ebenso gut realisierbar gewesen wären wie der statsächlicheく Verlauf. Diese Methode der Revision bzw. Umwertung, die bei Lommel anhand zahlreicher Beispiele aus Literatur und Film beschrieben wird, ${ }^{32}$ findet ihre Begründung wie von selbst, sozusagen zwanglos, durch die Erweiterung des Begriffs der Wahrnehmung auf die Rezeption von Literatur und die damit gegebene enge Verflechtung von Literatur/Kunst und Leben im Prozess der endlos autobiografischen Tätigkeit von Wahrnehmung. »Textwahrnehmung besteht aus Lebens-Ideen und nicht aus >Worten pur ; was sich durch Sprache ereignet, übersteigt in jedem Fall den Wortlaut; Literatur wird wahrnehmbar durch die Erweiterung des Konzepts der kognitiven und emotionalen Konstruktion von Wirklichkeit«, so Scheffer im gegebenen Zusammenhang.

Entwickelt sich dieses Vermögen immer weiter, so könnte es sich in seiner (Re-)Kombinationsfähigkeit soweit steigern, dass Möglichkeiten entstehen, die zuvor nicht einmal in Ansätzen gesehen wurden, um der subjektiv real erlebten Ausweglosigkeit zu begegnen, die unter Bedingungen der Echtzeit entstanden ist. Wobei sich Ausweglosigkeit hier versteht als Unmöglichkeit, sinnvoll anders zu handeln, als nur das immerfort Gleiche repetitiv im Sinne schlechter Unendlichkeit fortzusetzen bzw. zu wiederholen. Wenn dem so ist, dann wäre das an die Lektüre gebundene, kreative Vermögen der Neukombination gegebener Konstellationen ein (hoffentlich) wirksames Antidot gegen die herrschende Logik der technisch-digitalen Verschaltung des Körpers, damit der Wahrnehmung und des denkenden Bewusstseins, die objektiv jene Wirkungen zeitigt, von denen Baudrillard und Virilio gesprochen haben. Auf diese Weise könnte Pluralisierung, ungeachtet des schlechten Verständnisses im Sinn von Beliebigkeit, Indifferenz, Desinteresse nunmehr als Vielfalt verschiedener Lesarten eines literarischen Textes, wie der Verschiedenheit der

32 So für die Literatur etwa Borges' Der Garten der Pfade, die sich verzweigen, Cortazars Rayuela, Calvinos Wenn ein Reisender in einer Winternacht und für den Film Kieslowskis Der Zufall möclicherweise, Die zWei Leben der Veronika, Resnais' SMoking/No SMOKINC oder Tykwers LOLA RENNT. 
Weltwahrnehmung differenter Subjekte verstanden, ein befreiendes Potenzial entfalten. Mit dem Ziel, sich gegen die Logik des Gegebenen/Vorherrschenden, das diesmal nur ein technisch Erzeugtes wäre, durch Innovation(en) der Fantasie nicht nur zu behaupten, sondern es zu öffnen auf einen Horizont neuer Erfahrung, der zum gegenwärtigen Stand noch nicht eingeholt ist.

Genau in dieser Dynamik gibt sich neuerlich das Modell der Rekursion zu erkennen, das als allgemeiner Modus des Hervorbringens neuer Möglichkeiten fungiert, die schließlich zu Gegebenheiten werden, bis diese wiederum noch nicht dagewesenen, innovativen Entwürfen weichen müssen. Dieses Modell der Rückkopplung gilt umso mehr auch für die Selbstbestimmung der Subjekte, als nach dem Zerfall universaler resp. totaler Weltbildkonstruktionen als letzte Bezugsinstanz für Sinn nur das eigene Leben geblieben ist, ${ }^{33}$ mit allen Risiken der Selbsttäuschung, die bis zur Selbstschädigung reichen können, wenn sich das Subjekt ernsthaft verkalkuliert und die entstehenden Kosten nicht von einer Gesellschaft übernommen werden können, die mit Ulrich Beck ihrem Namen als Risikogesellschaft alle Unehre macht. ${ }^{34}$ Auch unter diesem Aspekt kann die Literatur sich als Gewinn für diejenigen erweisen, die im Leben (vorgeblich) nicht das erreicht haben, was sie eigentlich intendierten, da das bisher in dieser Sache falsch gelebte Leben ja auch nur eine realisierte Möglichkeit von vielen anderen denkbaren wäre. Jedoch setzt eine solche Betrachtungsweise voraus, dass die betroffenen Subjekte von sich aus ein Interesse an Einsicht in die eigenen Verfehlungen mitbringen oder $\mathrm{zu}$ entwickeln bereit sind, sobald es darum geht, den blinden Fleck der eigenen (Selbst-)Wahrnehmung aus einer anderen Perspektive anzusehen bzw. $\mathrm{zu}$ reflektieren und so Gewohnheiten und Mentalitäten sichtbar werden zu lassen, die man nicht ohne weiteres verändern möchte oder kann, weil sie das eigene Selbstbild geprägt haben. Auf diese Weise wären die mit Lommel und Scheffer im gegebenen Kontext fortgeführten Überlegungen zu Möglichkeitssinn und Lebensalternativen nicht nur eine Kompensation im psychologischen Sinn des Wortes, sondern könnten, sofern es objektiv nicht bereits zu spät dafür ist, zu einer Korrektur begangener Verfehlungen in der Wahl des Berufs oder eines Lebenszieles, sei dies weltanschaulicher oder erotischer Natur, beitragen. Dazu allerdings wäre die Rückgewinnung von Autonomie, zumindest von Teilautonomie in den betreffenden Punkten, nötig, weil das

33 Vgl. B. Scheffer: Interpretation und Lebensroman, S. 184.

34 Gemeint ist Ulrich Beck: Risikogesellschaft. Auf dem Weg in eine andere Moderne, 21. Aufl., Frankfurt a.M.: Suhrkamp 2012. 
Subjekt sonst nicht zum Träger seiner Handlungen wird, sondern, wie Mieth schreibt, »als Vollzugsorgan von Verhaltensschemata erscheint, die ihm selbst undurchsichtig bleiben. $«^{35}$

Wenn Autonomie mit Mieth des Weiteren verstanden wird als Eigengesetzlichkeit der Erfahrung, ${ }^{36}$ d.h. als Freiheit von heteronomer Bestimmung, so korrespondiert dies mit dem Autonomieaspekt der Positionen Text und Subjekt innerhalb des umfassenderen Netzwerks literarischer Kommunikation, von dem bereits die Rede war. Internalisierte Verhaltensschemata und die unbewusste resp. vorpersonale Orientierung an ihnen können nur dann korrigiert werden, wenn das Subjekt sich mit einer Instanz konfrontiert, die ihm die Alternative aufzeigt, sie ihm in Form einer (internen) Differenz wahrnehmbar werden lässt. Wenn wir dem Zwang, uns in Bildern und Vorstellungen zu bewegen, auch nicht entgehen können, ${ }^{37}$ so besteht doch die Möglichkeit, Vorstellungen und Bilder, die während der Lektüre wie außerhalb davon in fremden Kontexten (rückbezüglich und antizipatorisch) entstehen, nicht unreflektiert zu übernehmen, sondern sie vielmehr aposteriori mit der nötigen Distanz beständig aneinander zu korrigieren und auf diese Weise durch Konstruktion (via Identifikation) und Dekonstruktion (via Illusionsbruch) zu neuen Auffassungen/Erfahrungen von Welt und eigener Identität zu gelangen. ${ }^{38}$ Denn beides geht nur zusammen, d.h. in Wechselwirkung miteinander und nicht isoliert voneinander. So erscheint es denkbar, durch ständige Rekursion im fortgesetzten Prozess des bewussten internen Reflektierens wie weltzugewandten Interagierens mit zuvor als unveränderlich gegeben erscheinenden Dispositionen eigenen Denkens und Verhaltens, mit anderen Worten durch die Fähigkeit zur Uminterpretation von in diesem Sinn Gegebenem (Linke), zu neuen Einsichten und den damit verbundenen Verhaltensänderungen zu

35 D. Mieth: Moral und Erfahrung, S. 138.

36 Vgl. ebd.

37 Vgl. ebd., S. 199. Damit wird zudem angesprochen, dass das Subjekt, wie Andreas Reckwitz mit Lacan formuliert, erst durch den Anschluss an die symbolische(n) Ordnung(en), seien es Bilder oder Texte mit den daraus entstehenden Vorstellungen, »zur intelligiblen Instanz wird. «Andreas Reckwitz: Subjekt, Bielefeld: transcript 2008, S. 58. Dies bedeutet, dass jenes Subjekt außerhalb der symbolischen Ordnung - mit Bezug auf die Sprache spricht Reckwitz auch von der »Signifikantenordnung «-nicht existiert, sondern vielmehr von ihr erst hervorgebracht wird. Vgl. ebd. Mieth spricht in diesem Zusammenhang von einer Dialektik zwischen den vielen Bildern des Menschen und dem Menschen selbst, der jeder bildhaften Darstellung entzogen bleibt. Vgl. D. Mieth: Moral und Erfahrung, S. 199. 
gelangen. Dies bedeutet zugleich, dass es analog zum pluralistischen Menschenbild der Gegenwart auch nicht nur ein einzige Vorstellung vom eigenen Selbst gibt, sondern eine Vielfalt von bestehenden Möglichkeiten und Hinsichten, die im Rahmen einer Revision als neu rekombinierbar gedacht werden sollten. 



\section{Zur Interaktion von Jetztzeit und geschichtlicher Vergangenheit - Komplexes Erzählen in Alexander Kluges Lebensläufen}

Anders als im Fall der bisher besprochenen Autoren, geht es bei Alexander Kluge im Grunde nie um die Autopoiese der Identität des Schreibenden (verstanden im Sinn von Selbsterhaltung wie Veränderung) und deren Bezug zur Welt als Gegenstand des literarischen Textes. Fast alle von Kluges Erzählungen sind folglich nicht im gleichen Sinn selbstreferenziell wie die übrigen bisher behandelten Texte. So kommt es, dass im Unterschied dazu die Umstände der Entstehung seiner Texte nicht selbst in das Erzählen einfließen. Sie bleiben sozusagen außen vor und können deshalb nur als hypothetische Annahme mit dem empirischen Autor in Zusammenhang gebracht werden, sofern es sie nach dem bisher Gesagten zwar auch geben muss, ohne dass sie jedoch vonseiten des Autors bewusst in Betracht gezogen werden. Es fehlen aus diesem Grund in seinen Erzähltexten die Strukturen rekursiver Rückkopplung, die für das Schreiben der anderen Autoren prägend waren, und es lassen sich daher für seine Produktion die verschiedenen Modalitäten von Emergenz nicht in gleichem Maße reklamieren. Bleiben die Ermöglichungsbedingungen, die mit der Identität des empirischen Autors zusammenhängen - seiner Intentionalität und seinen Lebensumständen, seiner Biografie usw. -, außer Betracht, so kann per definitionem auch nicht davon die Rede sein, dass die Nullstelle des Diskurses in den manifesten Text der Erzählung, wie in den anderen Beispielen, mit hineingenommen und somit sichtbar werde.

Es wird damit an dieser Stelle offensichtlich, dass das Bezugssystem der erzählerischen Produktion Kluges, wie oben angedeutet, weniger in der von Ungewissheit, Selbstzweifeln oder durch Zugehörigkeit zu einer gesellschaft- 
lichen Minorität bedrohten, subjektiven Identität des Autor-Erzählers zu suchen ist, wenngleich der Stoff seiner Literatur stets auch gekoppelt ist an das eigene Erleben einer vergangenen und immer noch gegenwärtigen Zeit, der Zeit der Geschichte. Dies hat seinen Grund vermutlich darin, dass sich für ihn die Kategorie der Identität nur negativ, also im Sinne von Entzug und Mangel versteht. So schreibt er in Geschichte und Eigensinn gemeinsam mit Oskar Negt:

»Wenn wir von Identität sprechen, so geht es für deutsche Verhältnisse um das Bedürfnis, d.h. den Mangel an Identität. Wir haben die Kategorie bisher mehrdeutig verwendet. Einer kann sich identisch verhalten; dies ist aber die Summe seiner Schwankungen, Nicht-Identitäten, und eines Restes an Notwehr hiergegen, in dem sich beharrliches, identisches Festhalten an einem Rest oder einem Vorbehalt zeigt. [...] Soweit wir von Identität handeln, sprechen wir von einer Eigenschaftskette, die sich im Zustand radikaler Bedürfnisse befindet, also der Substanz nach: von Nicht-Identität. « ${ }^{1}$

Vor diesem Hintergrund wird verständlich, warum der Autor in seiner literarischen Praxis einen anderen Weg gegangen ist als den des selbstreferenziellen Erzählens. Während bei den zuvor behandelten Autoren das literarische Erzählen nicht nur als zurückgelegte Strecke der literarischen Verarbeitung eigenen Lebens, sondern auch durch seine innovativen Strukturformen spezifischer und allgemeiner Rekursivität auf die schockhafte Erfahrung von Nicht-Identität, dem damit verbundenen Sinnentzug, zu antworten sucht und somit als Entzogenes zur Quelle unabschließbarer Produktivität wird, ${ }^{2}$ entscheidet sich Kluge von Anfang an für Form und Perspektive heteroreferenziellen Erzählens.

Unter dem Gesichtspunkt von Komplexität scheint dies prima facie eine Reduktion zu bedeuten, denn damit gerät das Zugleich der Ebenen der Genese im Schreibprozess (mit allen Implikationen von Zeit, Ort und Intentionalität des Autors) sowie des intradiegetischen Geschehens mit den Rückkopplungen zur empirischen Wirklichkeit des Schreibenden außer Betracht. Somit stellt sich die Frage, was Kluges Schreiben im Zusammenhang von Komplexität und Literatur denn dann interessant macht. Der Ansatzpunkt

Oskar Negt/Alexander Kluge: Geschichte und Eigensinn. 7. Aufl. Frankfurt a.M.: Zweitausendeins 1983, S. 376.

2 Diese Erfahrung des Entzugs ist stets verbunden mit der Frage nach dem eigenen Sein: Wer bin ich denn dann eigentlich, wenn ich nicht bin? 
im gegebenen Kontext besteht zweifellos darin, dass sein narratives Verfahren Geschichte nicht als systemisches Kontinuum oder lineare Sukzession von Ereignissen mit den daran beteiligten Akteuren nimmt, sondern fragmentiert in eine Vielzahl von Geschichten, Episoden, theoretischen Einlassungen, die - bis auf wenige Ausnahmen - untereinander weder durch zugleich in ihnen auftretende Figuren noch vermittels durchgängiger Handlungsstränge oder deren Verzweigungen verbunden sind. Kontinuität und Kohärenz des erzählten Geschehens im Ganzen und der erzählten Figuren im Einzelnen als Prinzipien der klassischen Narration werden durch die konkrete Wahrnehmung zeitgeschichtlicher Ereignisse und der daran beteiligten Subjekte durchbrochen, die nicht mittels einer linearen Zeitvorstellung beschreibbar ist, da sie wesentlich instantan verläuft und simultan mit Erinnerungsinhalten interagiert. Die Unidirektionalität der abstrakten zeitlichen Sukzession, bestehend aus Vergangenheit, Gegenwart und Zukunft, die im klassischen Erzählen durch den Kunstgriff von Ana- bzw. Prolepse umgelenkt oder hypothetisch verlängert werden kann, erweist sich folglich für die erzählerische Umsetzung der Interaktion zwischen Jetztzeit und erlebter Vergangenheit, wie sie für Kluges Schreiben typisch ist, als unangemessen.

Dies ist nun der Punkt, wo die Subjektivität des empirischen Autors ins Spiel kommt, denn es sind letztlich seine Erfahrungen, die in den Texten zur Sprache kommen. Dass er sie selbst gemacht hat, wird u.a. in folgendem Hinweis an den Leser deutlich:

»Es hat den Anschein, dass einige Geschichten nicht die Jetztzeit, sondern die Vergangenheit betreffen. Sie handeln in der Jetztzeit. Einige Geschichten zeigen Verkürzungen. Genau dies ist dann die Ceschichte. Die Form des Einschlags einer Sprengbombe ist einprägsam. Sie enthält eine Verkürzung. Ich war dabei, als am 8. April 1945 in 10 Meter Entfernung so etwas einschlug. ॥ $^{3}$

Dies bedeutet mit anderen Worten nun auch, dass die Form der narrativen Adressierung, wie gesagt, zwar heteroreferenziell ist, sofern auf der Ebene der erzählten Figur(en) nicht explizit die Identität des empirischen Autors namentlich genannt und verhandelt wird, sondern literarisch erfundene Personen mit authentischen Anteilen auftreten, wie zu Beginn von Kluges Lebens- 
läufen vermerkt ist. ${ }^{4}$ Was den Erfahrungsgehalt betrifft, ist das Bezugssystem jedoch unverkennbar in der Subjektivität des empirischen Autors AK zu suchen, folglich auf unhintergehbare Weise mit seiner Identität verknüpft und so gesehen durchaus selbstreferenziell. ${ }^{5}$

Wenn dem aber so ist, resultiert daraus eine subjektive Perspektive auf das kollektive Geschehen des geschichtlichen Prozesses. Dies wiederum berechtigt dazu, Kluges erzählerische Strategie als eine des historischen Konjunktivs zu begreifen. Denn ihn interessieren als Subjekt der Geschichte weniger nationalstaatliche Gebilde mit den dazugehörigen Repräsentanten und Ethnien in ihrer objektiven Bestimmtheit, sondern individuelle Akteure, die als Figuren seiner Erzählungen den Zeitläuften ausgeliefert sind und stellvertretend für eine bestimmte Reaktionsbildung, eine bestimmte Haltung oder Mentalität angesichts von ihnen nicht oder kaum beeinflussbarer Umstände stehen, von denen also vorstellbar ist, dass es sie so wie erzählt auch wirklich gegeben haben kann. Daher lässt sich dieses Verfahren auch vor dem Hintergrund der allgemein gewordenen Simulation begreifen, indem die Literatur in der beschriebenen Weise mit möglichen Konstellationen der Figuren und Umstände darauf antwortet. ${ }^{6}$

Geht man von diesem Ansatz aus, dann ergibt sich bei Kluge - bezogen auf den geschichtlichen Prozess - durchaus eine Analogie zu dem, was im Zusammenhang der Sichtbarwerdung der Ermöglichungsbedingungen literarischen Erzählens als Migration der Nullstelle in den Diskurs benannt wurde, wenngleich anders als zuvor gedacht. So spricht er in vergleichbarer Weise

So heißt es in einem den Lebensläufen vorangestellten Kommentar, dass diese »teils erfunden, teils nicht erfunden [sind] «. Alexander Kluge: »10. Lebensläufe [1964]«, in: Ders.: Chronik der Cefühle, Bd. II (2004), S. 675.

Die Implikation der Biografie des Autors ist auch daran erkennbar, dass, wie in den Lebensläufen, nicht nur das berufliche Profil der erzählten Figuren oft im Gerichtsoder allgemeiner gesprochen im Rechtswesen angesiedelt ist (Scheliha, Korti), sondern auch viele Texte Rechtsfragen zum Inhalt haben (vgl. Anita C.), mit denen sich der Autor als promovierter Jurist und Justiziar am Frankfurter Institut für Sozialforschung bestens auskannte.

6 So spricht Thomas Combrink auch vom konstellativen Erzählen Kluges. Vergleichbar den Lebensvarianten bei Lommel ist damit eine Vervielfachung der Identität mit den Mitteln der Einbildungskraft gemeint: »Der Ausdruck konstellativ bezieht sich auf die Einsicht, daß wir gleichzeitig in mehreren Realitäten leben und daß es Aufgabe der Literatur ist, die Tektonik dieser Wirklichkeiten aufzuzeigen.«Alexander Kluge: Theorie der Erzählung. Frankfurter Poetikvorlesungen. Berlin: Suhrkamp 2013, Beiheft zur DVD, S. 18. 
von der »Null-Stellung des Reiches $\aleph^{7}$ und meint damit den Zeitraum zwischen Frühjahr 1945 und der Währungsreform, in dem das Land zerstört und die eigene Staatsgewalt verschwunden war. Der Begriff der Null-Stellung steht dabei für die Vorstellung einer temporären Außerkraftsetzung des Realitätsprinzips der Geschichte für das genannte zeitliche Intervall unmittelbar nach Ende des 2. Weltkriegs. ${ }^{8}$ Gemeint ist damit der Bedeutung nach, wie Kluge ausführt, »eine unerwartete Lücke in dem Drucksystem, das auf der Erfahrung liegt «, ${ }^{9}$ verbunden mit einer Vorstellung geschichtlicher Gegenwart und Zukunft, die sich von der Wiederherstellung mehr oder weniger identischer Zwangsverhältnisse, welche mit dem architektonischen und organisatorischen Wiederaufbau einhergingen, abwendet und in einem radikaleren Sinn neu anfangen möchte. Der Zerstörung des Textgewebes, von der Iser gesprochen hat, entspricht dabei das Aussetzen, die Unterbrechung, einer sich gleich oder ähnlich fortsetzenden Kontinuität des geschichtlichen Verlaufs zugunsten des Einspruchs, dass zumindest der aktive Teil der Bevölkerung die Zeit der offenen Möglichkeiten für eine grundlegende Änderung der Verhältnisse zum Besseren hätte nutzen können. Dies wäre durch Änderung der eigenen Lebensführung, d.h. Neuorientierung im Lebenslauf, zusammen mit alternativen Formen der Sozialisierung denkbar gewesen. ${ }^{10}$

Sichtbar wird somit, ähnlich wie im Fall der Interaktion von Input und Output im kybernetischen Regelkreis, eine Kaskade von Möglichkeiten, also Möglichkeiten der Neukombination von in der Realität bereits vorhandenen, zumindest angelegten Optionen des Handelns. Kluge fokussiert damit vorderhand auf das Handeln empirischer Subjekte, das sich insofern als allgemein begreifen lässt, als es in der Summe geschichtlich determiniert ist, also den jeweils herrschenden Verhältnissen weitgehend unterliegt. Selbst wenn es so etwas wie die subjektive Erfahrung der einzelnen Individuen geben mag, die sich in der Besonderheit, möglicherweise auch Unverwechselbarkeit ihrer

7 O. Negt/A. Kluge: Ceschichte und Eigensinn, S. 378.

8 Vgl. ebd. S. 379.

9 Ebd.

10 Die Bedeutung dieser dann wieder vertanen Möglichkeit für das Verständnis deutscher Ceschichte kommt auch im folgenden Kommentar zum Ausdruck: »Lebensläufe wenden sich, die kollektiven Bewegungen fallen zu den alten Figuren zusammen. Ohne dieses san sich und nicht >für uns` geöffnete Fenster dieses Null-Punkts ist aber deutsche Geschichte nicht zu erfahren. « Ebd. In ähnlicher Weise dürfte dies für die Nachwendezeit ab 1990 zutreffen. 
Lebensläufe zu erkennen gibt, geht Kluge davon aus, dass individuelle Entscheidungsverkettungen, wie sie sich in Lebensläufen manifestieren, durch gattungsgeschichtliche Voraussetzungen in einer Weise vorgeprägt sind, dass die kritische Betrachtung davon nicht dispensieren kann und folglich der Gesichtspunkt des Allgemeinen den des individuell Besonderen als Quantité négligeable erscheinen lässt. Gemeint sind hier die evolutionsgeschichtlichen Ermöglichungsbedingungen individueller Erfahrung, die soweit zurückreichen wie die Geschichte der Menschheit. ${ }^{11}$ Es kommen auf diese Weise anthropologisch bestimmte Zeithorizonte ins Spiel, die aus dem Begriff Geschichte ein komplexes Geschehen mit »einander mehrdimensional überlappenden Geschichtsverläufen ${ }^{12}$ machen. Dabei wird nun die Polysemie des Wortes 'Geschichte erkennbar, das, wie in der folgenden Beschreibung je nach Verwendung bzw. Kontext, eine andere Konnotation erhält:

»Man betrachte nur einen Ausschnitt, der für sich eine Geschichte darstellt-andere Geschichten wären zum Beispiel Hammer, Pflug, Bombe, Familie, Staat, die Beerdigung, die Sexualität, die Sprache -: eine Stadtland-

11 Hier kreuzen sich Human- und Naturgeschichte, die mit jeweils unterschiedlicher Logik den Menschen als sich selbst hervorbringendes, kulturelles Wesen bzw. als Produkt der biologischen Evolution betrachten. In jüngerer Zeit hat sich für den wachsenden Einfluss des Menschen auf die Umwelt, verbunden mit einem immer weiter zunehmenden Grad an Selbstwirksamkeit, der Begriff des Anthropozäns eingebürgert, der von Paul Cruzen und Eugene Stoermer um die Jahrtausendwende in die Diskussion gebracht wurde. Vgl. https://de.wikipedia.org/wiki/Anthropoz\%C3\%A4n vom 27.12.2020. Er ist dann im deutschen Sprachraum besonders von Peter Sloterdijk, im Sinne der anthropotechnischen Wende, aufgegriffen und vor allem in der Untersuchung Du mußt dein Leben ändern. Über Anthropotechnik (2009) in Hinsicht auf die Selbstbildung resp. Selbstwirksamkeit alles Humanen durch Techniken des Übens ausgebaut worden. Dieser Selbstbezug wird auch bereits von Cramer thematisiert: »Mit dem technischen Zeitalter seit hundertfünfzig Jahren und besonders mit dem Eintritt in das biotechnische Zeitalter seit zehn Jahren tritt erstmalig eine bis dahin nicht gekannte Interaktion zwischen dem Reich der Ideen (Poppers Welt III) und der Natur (dem Reich der Evolution) auf. Diese neuartige, vom Menschen hervorgebrachte und von ihm zu verantwortende Rückkopplung kann der Naturgeschichte die gleiche Instabilität, den gleichen Komplexitätsgrad, die gleiche Krisenanfälligkeit aufprägen, wie wir sie in der Geschichte beobachten. Diese Wechselwirkung droht außer Kontrolle zu geraten und zur globalen ökologischen Katastrophe oder zum Atomtod oder zur genetischen Totalmanipulation zu führen.«F. Cramer: Chaos und Ordnung, S. 293.

12 O. Negt/A. Kluge: Ceschichte und Eigensinn, S. 783. 
schaft z.B. ist 5000 Jahre alt, also jünger als die sog. historische Geschichte, aber um eben 5000 Jahre kompakter als ein Lebenslauf. ${ }^{13}$

Schon auf sprachlicher Ebene ist somit das in Frage stehende Phänomen nicht einheitlich und zu erwarten ist, dass es sich empirisch noch viel inhomogener und disparater, und zumindest aus Sicht der handelnden, darin verwickelten Subjekte fundamental komplex und entsprechend inkohärent gestaltet. Damit Geschichte in ihrer Prozesshaftigkeit intelligibel werden kann, braucht es nicht nur die Sprache, sondern den begrifflichen Diskurs mit seinen definitorischen Bestimmungen, der andererseits das Phänomen in seiner überwältigenden Totalität an Fülle, Vielfalt, aber auch Widersprüchlichkeit, gerade aufgrund seiner an Finalität bzw. Teleologie orientierten begrifflichen Zurichtung verfehlt. ${ }^{14}$

Dies ist der Grund dafür, warum Kluge im Vorwort seiner Chronik über die >Unheimlichkeit der Zeit [1977] davon spricht, es handle sich im Folgenden um »Geschichten ohne Oberbegriff «. ${ }^{15}$ Was ihn in seiner literarischen Arbeit interessiert, ist darum, wie Menschen als individuell handelnde Akteure mit dieser nicht vorhersehbaren, sie in ihrer Existenz oft bedrohenden, widersprüchlichen Pluralität umgehen, wie sie sich angesichts schwieriger, mitun-

(1)
tersuchten dynamischen Systeme mit uneinheitlichen Zeitbegriffen arbeiten. Die historische Zeit ist kaum vergleichbar mit den in Physik, Chemie, Biologie oder den Sozial-, Finanz- und Wirtschaftswissenschaften geltenden zeitlichen Parametern. Wesentlich gemeinsam ist ihnen jedoch Instabilität, d.h. grundlegende Unvorhersehbarkeit, und nicht-lineare Dynamik. Dies sind dann die Hinsichten, unter denen sie in der Komplexitätsforschung betrachtet werden. Zudem gilt nach allem bisher Gesagten, dass es die eine, allgemeingültige Theorie der Ceschichtsschreibung nicht mehr geben kann. Dies ergibt sich aus dem Grundsatz, dass Zunahme von Komplexität sich immer auch auf die Strategien ihrer Bewältigung auswirkt, indem diese ihrerseits komplexer werden (müssen). Vgl. dazu Kap. III der vorliegenden Arbeit. Pluralität bedeutet dann in diesem Zusammenhang die Arbeit mit unterschiedlichen theoretischen Ansätzen, die ein identisches Phänomen unter variablen Aspekten visieren. In diesem Sinn formuliert auch Cramer: »Die Frage, ob überhaupt eine allgemeine Theorie der Geschichtswissenschaft möglich ist, läßt sich wissenschaftlich nicht entscheiden. [...] Das heißt aber: Historische Strukturen und Theorien können in pluralistischer Weise nebeneinander bestehen, in gleicher Wertigkeit, friedlich ko-existierend. Es gibt keinen Anspruch, der aus einer einzigen historischen Theorie abgeleitet werden könnte.« F. Cramer: Chaos und Ordnung, S. $290 \mathrm{f}$. 
ter auswegloser politischer und historischer Verhältnisse, für die sie selbst nichts können, verhalten. Wichtig ist ihm dabei, dass die im Textausschnitt beispielhaft genannten Prozesse von Geschichte »durch konkrete Menschen innerhalb ihrer Lebenszeit « »wiederangeeignet werden [können] «. ${ }^{16}$ Gelingt das nicht, dann rächt sich die Geschichte sozusagen als objektiver Vollzug undurchschauter Zusammenhänge und Wirkursachen am Subjekt, ${ }^{17}$ dem es verwehrt bleibt, die einmal auf Ebene der Gattung (allgemein) gemachte Erfahrung zu rezipieren, also davon als Individuum Kenntnis zu nehmen. ${ }^{18}$ Aufgabe und Funktion des Erzählens kann nun darin gesehen werden, die in den Texten - vermittelt über die Subjektivität des Autors - zu Buche schlagenden allgemeinen Erfahrungen an den aufgeschlossenen Leser weiterzugeben. Dies geschieht auf dem Weg der erzählerischen Signifikation eines fiktiven oder halb fiktiven Geschehens in historisch realen Kontexten, wo, wie anhand der Lebensläufe erkennbar, mindestens zur Hälfte fiktive Personen handeln.

Dieses Verfahren wirkt plausibel, wenn man bedenkt, dass angesichts der Komplexität des geschichtlichen Prozesses Kohärenz (Einheit) und Kontinuität (Stetigkeit) subjektiver Identität, wie sie sich in >normalisierten< Lebensläufen ausdrücken, als Fiktion erscheinen müssen. Dies gilt in gleichem Maße für das Kollektiv, zu dem ein betreffendes Subjekt gehört, z.B. dem deutschen Staatsvolk. ${ }^{19}$ Ist aber der lückenlose Zusammenhang, die vermeintliche Einheit des Subjekts - seine Identität -, in der Empirie gar nicht vorhanden, sondern nur als heterogenes Konglomerat aus Selbstprojektionen, einer Pluralität von Einflüssen aus Umwelt und Gesellschaft sowie bewussten Strebungen des intentionalen Willens existent, so leuchtet es ein, dass erst durch Erzählen die Einheit von Identitäten, nämlich narrativ, hergestellt werden kann, die es als substanzielle im Grunde schon lange nicht mehr gibt. Nicht zuletzt beruht diese Fähigkeit der Erzählung auf der anfangs benannten, allgemeinen Eigenschaft der Narration, Zusammenhänge auch dort noch sinnfällig herstellen zu können, wo das wissenschaftliche Denken aufgrund nomothetischer Einschränkungen die Segel streichen muss. ${ }^{20}$

Auch für Kluge stellt sich nun die Frage, wenngleich in anderer Terminologie, nämlich der Dialektik des historischen Materialismus, ob die im Zu-

\footnotetext{
16 O. Negt/A. Kluge: Ceschichte und Eigensinn, S. 783.

17 Vgl. dazu die Vorbemerkung in vorliegender Studie.

18 O. Negt; A. Kluge: Ceschichte und Eigensinn, S. 783.

19 Vgl. ebd., S. 785.

20 Vgl. dazu Kap. I in vorliegender Studie.
} 
sammenhang komplexer dynamischer Systeme unter Bedingungen der Instabilität eintretende Verzweigung der Zustandsentwicklung (Bifurkation) ${ }^{21}$ auf den geschichtlichen Prozess übertragbar ist. Dabei geht er zunächst davon aus, dass die sich dynamisch verändernden Komponenten des geschichtlichen Prozesses (objektiv) sich als Wirkursachen zu den Gründen der innerhalb von individuellen Lebensläufen stattfindenden Veränderungen (subjektiv) inkommensurabel verhalten. Damit erklärt er das Auseinanderfallen der Entstehung individuellen Bewusstseins über den Stand der historischen Entwicklung zu einem gegebenen Zeitpunkt und der Möglichkeit, durch entsprechende Maßnahmen kollektiver Organisation dem sich abzeichnenden historischen Verlauf eine andere Wendung zu geben als die, welche dann faktisch eintritt. ${ }^{22}$

Wie man sich dies vorstellen kann, erläutert er aus Gründen der Anschaulichkeit in Geschichte und Eigensinn am Beispiel einer fiktiven Lehrerin, die im Frühjahr 1945 in einem Keller Zuflucht vor einem britischen Fliegerangriff sucht. ${ }^{23}$ Dabei geht es letztlich um die Frage, ob und wie das konkrete Unten (Lehrerin im Keller) gegen das Oben (Bombergeschwader) als Standpunkte im geschichtlichen Prozess - und die mit diesen Positionen verbundenen geschichtlichen Kräfteverhältnisse - umgekehrt werden kann. Dazu schreibt Kluge:

»Die letzte Chance der Organisierung von Cegenwehr hätte für die Lehrerin wahrscheinlich 1928 bestanden, wenn sie sich damals organisiert hätte: mit anderen Lehrerinnen, Lehrern, Eltern, Kindern, ganzen gesellschaftlichen Klassen, nämlich denjenigen, die an einem Geschichtsverhältnis arbeiten, das nach diesem Zeitpunkt nur noch wie eine Automatik auf das Jahr 1945 hinzueilen scheint. Vielleicht wäre es damals möglich gewesen, die Geschichte noch zu wenden. Es ist ein Quantum an soziologischer Phantasie notwendig, um sich das überhaupt vorzustellen. [...] Es ist ein noch höheres

21 Vgl. dazu K. Mainzer: Komplexität, S. 118 (Clossar).

22 Vgl. O. Negt/A. Kluge: Geschichte und Eigensinn, S. 788.

23 Unwillkürlich denkt man hier an die Geschichtslehrerin Gabi Teichert gespielt von Hannelore Hoger in DiE PATRIOTIN (BRD 1979, R: Alexander Kluge) oder an die Volksschullehrerin Gerda Baethe aus »Heft 2. Der Luftangriff auf Halberstadt am 8. April 1945«, in: Kluge, Chronik der Gefühle, Bd. II (2004), S. 27-82, hier S. 43 ff. In beiden Fällen handelt es sich um das, was Kluge mit Bezug auf die geschichtlichen Kräfteverhältnisse als »Strategie von unten« bezeichnet hat. Ebd., S. 43. 
Quantum an kooperativer Zusammenfassung der Arbeitsvermögen erforderlich, um an der Abwendung eines solchen Verhältnisses zu arbeiten. [...] Der Grund für das Motiv, sich zu organisieren, wird aus eindeutigen Wahrnehmungen im Jahr 1945 geliefert. Die objektive Möglichkeit, sich zu organisieren, besteht $1928 .{ }^{24}$

Das reale Auseinanderfallen der beiden Momente (1) Wahrnehmung der gegenwärtigen Situation (Bewusstsein) und (2) Möglichkeit, den katastrophischen Prozess des realgeschichtlichen Verlaufs abzuwenden (Organisationsfrage), wird damit sinnfällig. Wären Maßnahmen kollektiver Organisation, so Kluge, bereits vor dem Schwarzen Freitag von 1929 erfolgt, ${ }^{25}$ hätte in der Lücke von etwas mehr als eineinhalb Jahrzehnten an Veränderungen zum realen Verlauf gearbeitet werden können, sodass am Ende nicht die Katastrophe von 1945 hätte eintreten müssen. Die Frage aber, ob der Eintritt in eine katastrophische Entwicklung von Personen wie der Lehrerin zu diesem Zeitpunkt schon erkennbar gewesen ist, negiert Kluge mit Einschränkung: "Sie kann im Verhältnis zu den Massenbewegungen im Innern des Landes, zur Geschwindigkeit der Protestgefühle, oder zur Bewegungsform der englischen oder französischen Gesellschaft nicht ohne weiteres ermitteln, daß die Gesamtheit dieser Bewegungen dem Nationalsozialismus und dem Zweiten Weltkrieg zueilt. ${ }^{26}$

Es ergibt sich daraus im Umkehrschluss, dass die zeitlich weit auseinanderliegenden Momente nur unter der Bedingung, dass man sie in eine enge Kooperation bringt, zu einer Verkehrung der geschichtlichen Verhältnisse hätten führen können. Wenn man, wie Kluge, die Positionen von Unten und Oben als Stellungen im Geschichtsverhältnis begreift, »wo die gleichen Kräfte, die dieses Verhältnis erzeugen, in die Gewalt des von ihnen produzierten geraten ${ }^{27}$ so wäre die Möglichkeit einer Umkehr der geschichtlichen Kräfteverhältnisse bei gleichzeitiger Vermeidung eines katastrophischen Verlaufs nur für den Fall denkbar, dass das Bewusstsein nicht um Jahrzehnte hinter der realen Entwicklung zurückbleibt, sondern umgehend, wie bei einer kybernetischen Rückkopplung, mit der sich abzeichnenden Tendenz wirksam

\footnotetext{
24 O. Negt/A. Kluge: Geschichte und Eigensinn, S. 788.

25 Vgl. ebd., S. 787.

26 Ebd.

27 Ebd., S. 789.
} 
$\mathrm{zu}$ interagieren vermag. Für »[d]ie objektive Möglichkeit, sich zu organisieren «, ${ }^{28}$ um gegebenenfalls intervenieren zu können, müssten aber politische Bedingungen vorausgesetzt werden, die eine zeitnahe Interaktion zwischen der gesellschaftlichen Basis (Unten) und der administrativen Ebene (Oben) möglich machen. Es müssten also entsprechend flache Hierarchien vorhanden sein, die selbst unter gegenwärtigen Verhältnissen kaum irgendwo realisiert sind. Neben dieser politischen Vorbedingung, die im Grunde nur als ihr Resultat begriffen werden kann, wäre die zeitliche Umkehr geschichtlicher Kräfteverhältnisse damit nur für den Moment realisierbar, wo im Sinn rückläufiger Kausalität die Wirkungen dieser Kräfteverhältnisse zeitlich rekursiv mit ihrem eigenen Ursprung (den »Organisationsweisen des materiellen Grundverhältnisses « ${ }^{29}$ als Ursache) in Interaktion gebracht werden könnten, und dies zu einem Zeitpunkt, wo ein chaotisch komplexer Verlauf mit katastrophischen Folgen sich abzeichnet, aber noch vermeidbar ist. Andernfalls wäre es dazu einfach zu spät. ${ }^{30}$ Ein vergleichbares Szenario ist gegenwärtig mit der globalen Klimaerwärmung gegeben, wenngleich erste Gegenmaßnahmen bereits getroffen sind.

Was sich damit in recht überraschender Weise abzeichnet, ist, dass sich neben der Möglichkeit des erfolgreichen Transfers ${ }^{31}$ von Begriffen aus der lin-

28 Ebd., S. 788.

29 Ebd., S. 789.

30 Um diesen abstrakten geschichtstheoretischen Zusammenhang anschaulicher zu machen, entlehnt Kluge als Analogie aus der Gravitationslehre den Begriff des abarischen Punktes, der genau den Moment bezeichnet, wo sich die Gravitationskräfte von Erde und Mond aufheben. Diesen Punkt im Schwerefeld zwischen den beiden Himmelskörpern versteht er analog zum Moment zeitlicher Umkehr im geschichtlichen Denken. Es handelt sich, mit anderen Worten, um den Zeitpunkt, wo auf der Bahn eines bewegten Körpers von der Erde zum Mond sich für kurze Zeit die wirkenden Kräfte aufheben und auf diese Weise - wie kurzzeitig immer-ein Moment von Freiheit entstehen kann, das Kluge, wie erörtert, auch in herausgehobenen geschichtlichen Situationen als Potenzial für gegeben hält. Vgl. ebd., S. $789 \mathrm{f}$.

31 Es liegt nahe, den Transfer semantischer Inhalte aus dem Kontext des dialektischen Materialismus in die Begriffswelt komplexer Systeme damit zu erklären, dass die Aufrechterhaltung bzw. Schleifung der Antagonismen im geschichtlichen Prozess formal gedacht auf struktureller Ebene dem hoch geordneten Zustand eines komplexen Systems vergleichbar ist, das durch Störungen instabil werden kann. Ein solches weit vom Gleichgewicht entferntes System gerät an Instabilitätspunkten (Bifurkationen) in eine durch Ungleichgewicht geprägte Dynamik, die durch Input-Output-Rückkopplungen mit der Systemumgebung zu Phasenübergängen in andere Zustandsformen bis hin zum indeterministischen Chaos führt. Vgl. dazu den Abschnitt »Komplexität und Dy- 
ken Theorie materialistischer Dialektik in die Sprache komplexer nicht-linearer Dynamik gerade das Abarbeiten an der Frage, wie sich die Zirkelhaftigkeit der Denkfigur rückläufiger Kausalität, die dem linearen Geschichtsdenken als Petitio principii (Zirkelschluss) erscheinen muss, vermeiden ließe, zu Einsichten in den fundamental komplexen Charakter des Zusammenhangs zwischen menschlicher Wahrnehmungsfähigkeit und geschichtlichem Verlauf führt. Denkt man an dieser Stelle weiter, so wird klar, dass die offenbare Petitio principii nicht als Fehler oder Missgeschick, sondern als neuer Modus der Operationalität geschichtlichen Denkens begriffen werden muss, sobald man die Ebene bloßer Faktizität verlässt, um kontrafaktische, die Bahnen der zu einem gegebenen Zeitpunkt vorherrschenden Handlungsweisen konterkarierende Organisationsformen alternativen Handelns und die damit verbundenen Kaskaden von Möglichkeiten des zukünftigen Verlaufs, sprich alternativen Chronologien, in Betracht zu ziehen.

Es zeigt sich hier abermals, dass Modalitäten von Emergenz wie Nullstellung des geschichtlichen Verlaufs (analog zur Nullstelle bei Iser), Mehrstelligkeit der Komponenten (geschichtliche Kräfteverhältnisse des Oben und Unten sowie deren Voraussetzungen in den materiellen Grundverhältnissen) und rückläufige Kausalität (nachträgliche Veränderung fixierter geschichtlicher Kräfteverhältnisse) auch im Geschichtsprozess erkennbar sind. ${ }^{32}$ Darüber hinaus wird einsichtig, dass neben der Kontextsensitivität des Begriffs ,Geschichte<, verstanden im Sinn eines komplexen, mehrdimensionalen, mit Kaskaden von Möglichkeiten verbundenen Geschehens, die teilnehmende Beobachtung des Erzählers in Kluges Lebensläufen eine weitere Voraussetzung

namik«, in: Mainzer, Komplexität (2008), S. 38-51, hier S. 38-44. Analog dazu kann die Dynamik des geschichtlichen Prozesses mit seinen Krisen (Instabilitätspunkte) und katastrophischen Entwicklungen (indeterministisches Chaos) erklärt werden.

Dies lässt sich sogar, wie man bei Iser lesen kann, für die menschliche Naturgeschichte sagen, indem nämlich die Befreiung der Hand von der Fortbewegung als Effekt des aufrechten Gangs bei den Hominiden im Sinn eines generativen Einschnitts als frühester Modus von Emergenz begriffen werden kann (vgl. W. Iser: Emergenz, S. 38 f.). Wie Iser weiter ausführt, resultiert aus diesem Sprung in der Evolution »die Exteriorisierung menschlicher Organe (ebd., S. 39), die sich von der Hand über die Muskeln bis hin zum Cehirn fortsetzt (vgl. ebd., auch für die folgenden Ausführungen). Indem somit menschliche Organe zur Vorlage von Modellen werden, die es gestatten, über den eigenen Körper hinaus durch extrapolative Erweiterung »auf Umwelt auszugreifen« (ebd.), erweist sich zudem, dass auch die Erzeugung der kulturellen Umwelt seit der Zeit des frühen Menschen durch das Modell emergenten Hervorbringens zuvor nicht dagewesener Eigenschaften und Fähigkeiten der Gattung Homo erklärbar ist. 
dafür bildet, dass komplexe Zusammenhänge, die sich als globale der Beschreibbarkeit weitgehend entziehen, im Medium der literarischen Erzählung zur Anschauung gebracht werden können. So geht Kluge von den konkreten Erfahrungen einzelner Subjekte aus, die als Figuren seiner Erzählungen sich angesichts gesellschaftlicher und beruflicher Missstände Anfang der 6oer Jahre (dies gilt für Anita G., Manfred Schmidt, Schwebkowski und Korti) sowie den Wirren der geschichtlichen Verhältnisse in der Kriegs- und unmittelbaren Nachkriegszeit (Oberleutnant Boulanger, Kriminalrat Scheliha, Fräulein v. Posa und Eberhard Schincke) in bestimmter Weise verhalten und dadurch charakterisierbar sind.

Der beim Leser entstehende Eindruck ist folglich nicht der eines umfassenden zeitgeschichtlichen Panoramas, vor dessen Hintergrund die Figuren agieren würden. Vielmehr wird, wie im Fall der Anita G., ein Einzelschicksal in seinen entscheidenden Wendungen geschildert oder das Porträt von Respektspersonen, wie dem Amtsgerichtsrat Korti, bis in psychologische Einzelheiten der beruflichen und privaten Person gezeichnet. Bleiben die einzelnen Episoden, die im Stil parodistisch zwischen Anekdote und Reportage changieren, aufgrund ihrer Geschlossenheit, d.h. Zentriertheit auf die in ihnen geschilderte Person, ohne weitere Anschlussfähigkeit untereinander, so besteht ihr Zusammenhang allein in der chronikalen Zugehörigkeit zur gleichen Formation von historischer Zeit, als deren literarischer Ausdruck sie in Kluges Erzählungen figurieren. Es wird auf diese Weise sichtbar, wie sich Geschichte als Abstraktum durch gesellschaftliche Strukturen hindurch in Verhalten und Charakter einzelner Subjekte konkretisiert. Das Raster der kriminalistischen und strafrechtlichen Zusammenhänge, das in vielen Geschichten das Verhalten der Figuren determiniert, sei es nun passiv als Flucht davor (Anita G.) oder aktiv als Medium beruflicher Handlungsfähigkeit (Korti), wirft zugleich die Frage nach der Möglichkeit alternativen Verhaltens/Handelns auf. Diese wird im Erzählen selbst nicht beantwortet, stellt sich aber dem Leser implizit als Folge der Konsequenz des Dargestellten, seiner Ausweglosigkeit nämlich, die sich auch in Form des Nicht-anders-Könnens oder -Wollens der Figuren manifestiert.

Diese narrative Strategie wird wiederum vor dem Hintergrund des theoretischen Beitrags von Kluge/Negt verständlich, dem sich entnehmen lässt, wie das Wissen über geschichtliche Verläufe und den gesellschaftlichen Ursachen dafür so umgesetzt werden könnte, dass katastrophische Tendenzen frühzeitig erkannt und ihnen entgegengewirkt werden kann, bevor sie sich unumkehrbar verstetigen. Das Mittel der Verständigung darüber wäre neben 
der wissenschaftlichen Expertise das Erzählen in Form von Lebensläufen, das jedoch nur dann seinen Sinn zu erfüllen vermag, wenn es über die Zeit hinweg die Generationen miteinander verbindet. So müssen die einmal vom Kollektiv gemachten Fehler nicht immer von neuem wiederholt werden, sondern können als in den Lebensläufen kristallisierte Erfahrungen an die nächsten Generationen weitergegeben werden. Auf diese Weise kann bei den Rezipienten ein Lernprozess initiiert werden, der, sofern diese sich dem adressierten Kollektiv zugehörig erkennen, auf kognitiver Ebene bestimmte Haltungen, Mentalitäten, Verhaltensweisen zu identifizieren, kritisieren und womöglich vermeiden hilft, die in der deutschen Geschichte des 20. Jahrhunderts in ihren politischen Konsequenzen zu desaströs katastrophischen Entwicklungen geführt haben. Man kann sich diesen Lernprozess wieder so vorstellen, dass es in der Rezeption zur kognitiven Interaktion zwischen den in der Erzählung erscheinenden zeitgeschichtlichen Hintergründen sowie den darin auf je individuelle Weise verwickelten Figuren und im Familiengedächtnis der Lesenden abgespeicherten Inhalte kommt (Geschichten von Angehörigen, etwa den Großeltern oder Eltern, die innerhalb der Familien an die Generation der Kinder weitergegeben werden). ${ }^{33}$

Der eigentliche Lerneffekt würde nun darin bestehen, dass die Lesenden sich darüber klar werden, wie eine verhängnisvolle Entwicklung aus Umständen resultieren kann, die von den Betroffenen selbst nicht bestimmt und daher auch nicht verantwortet werden können. Es sind dies Umstände, die individuell als Zufälle erlebt werden, die aber, wie am Beispiel des Lebenslaufs der Anita G. deutlich wird, unter gesellschaftlich-ökonomischen Bedingungen, die für das gesamte Kollektiv gelten, in einen für die Betroffene verhängnisvollen Verlauf münden, indem ihre Bedürfnisse mit der geltenden Rechtsordnung kollidieren. Aus der Umgebung von Leipzig kommend, begeht Sie auf ihren Reisen im Westen fortgesetzt Eigentumsdelikte aus Geldnot, entzieht sich der Strafverfolgung durch Flucht, wird durch ein flüchtiges Verhältnis schwanger und stellt sich schließlich, als es nicht mehr anders geht,

Das Familiengedächtnis, das sich generationenübergreifend innerhalb der Familie durch Erzählen ausbildet, ist als Phänomen von der Sozialpsychologie untersucht worden. In Deutschland ist es vor allem im Kontext der Tradierung von Erinnerungen aus der Zeit des Nationalsozialismus zum Gegenstand von Forschungsprojekten geworden, wie Tradierung von Geschichtsbewusstsein unter der Leitung von Harald Welzer. Vgl. dazu die Kapitel »2. Familiengedächtnis« und »8. Erinnern und weitergeben«, in: Harald Welzer et al., »Opa war kein Nazi«. Nationalsozialismus und Holocaust im Familiengedächtnis, 9. Aufl., Frankfurt a.M.: Fischer 2015, S. 18-43 und S. 195-204. 
in hochschwangerem Zustand der Polizei. ${ }^{34}$ Evident ist dabei, dass die Figur aufgrund ihrer Empfindlichkeit, ihrer affektiven und materiellen Bedürfnisse zum Opfer der Verhältnisse wird, die sich in dem Moment gegen sie wenden, wo sie bestehende Verpflichtungen, zumeist Mietverhältnisse, verlängert, ohne dafür aufkommen zu können. Dass sie selbst gewillt ist, sich juristisch aus der Affäre zu ziehen, zeigt sich an dem Bemühen, einen Anwalt zu suchen, das jedoch schon beim Versuch der Kontaktaufnahme scheitert.

Es wird somit deutlich, dass die Möglichkeit $\mathrm{zu}$ alternativem Handeln/Verhalten, das auf einen besseren Ausgang der einmal verhängnisvoll verlaufenden Geschichte hinauswollte, nicht auf der Ebene des individuellen Subjekts der erzählten Figur zu suchen ist, sondern in den gesellschaftlichen Bedingungen, die einen übermächtigen Zwang auf die Betroffene ausüben. Veränderungen, zumindest deren Ermöglichung, müssten folglich auf dieser Ebene angedacht werden. Überlegungen dieser Art sind es, die als Lernprozess während der Rezeption, diese begleitend, ablaufen können. Nun soll ein Lernprozess, indem er, wie hier geschehen, deskriptiv erörtert wird, nicht als solcher den Rezipienten vorgeschrieben werden, sondern müsste im Sinn kommunikativen Handelns nach Habermas, sind die Voraussetzungen dafür tendenziell gegeben, gewissermaßen von selbst resultieren. ${ }^{35}$ Dies erscheint in dem Moment denkbar, wo der literarische Text nicht im Sinn irgendeiner vorgegebenen Ordnung aufgefasst werden soll, sondern gemäß den Regularitäten, die sich aus der kognitiven Aktivität der Rezeption mit ihrer Fähigkeit zur innovativen Erzeugung von Sinn und Bedeutung ergeben. ${ }^{36}$ So ist mit

Zum Inhalt der Erzählung Anita G. vgl. A. Kluge: Chronik der Gefühle, Bd. II, S. 734-748. Habermas selbst hat dies allerdings einschränkend nur für eine ideale Sprechsituation gelten lassen und eingeräumt, dass nur unter der Bedingung einer allgemeinen Symmetrieannahme »der eigentümlich zwanglose Zwang des besseren Arguments« seine Wirkung entfalten könne. Jürgen Habermas: »Vorbereitende Bemerkungen zu einer Theorie der kommunikativen Kompetenz«, in: Jürgen Habermas/Niklas Luhmann (Hg.), Theorie der Cesellschaft oder Sozialtechnologie - Was leistet die Systemforschung?, Frankfurt a.M.: Suhrkamp 1971, S. 101-141, hier S. 137. Zit. nach Michael Niehaus: »Das bessere Argument. Eine Anmerkung zur Logik des Argumentierens bei Jürgen Habermas«, in: Philosophisches Jahrbuch 105 (1998) H. II, S. 412-422, hier S. 413, https://philosophisches-jahrbuch.de/wp-content/uploads/2019/04/PJ105_S412-422_Ni ehaus_Das-bessere-Argument.pdf vom 18.11.2020.

Diese Ausführungen verstehen sich vor dem Hintergrund gleichsinniger Überlegungen, die Francisco Varela mit Bezug auf die Fähigkeit der Kognition zur autopoietischen Genese von Sinn und Bedeutung im Gegensatz zur Vorstellung der abbildhaften Übernahme vorgegebener Information anstellt. Vgl. Francisco J. Varela: Kognitionswissen- 
Fischer-Lichte letztlich von einer Unverfügbarkeit emergenter Rückkopplungsprozesse auszugehen. ${ }^{37}$ Andernfalls würde der Prozess dazulernenden Erkennens nicht wirklich befördert, sondern durch bloßen Nachvollzug nur kopiert und dadurch möglicherweise sogar behindert oder gar blockiert. Er soll deswegen wiederum nur im Sinne einer Möglichkeit - einer denkbaren, möglichen Interpretation - den Lesenden nahegelegt werden. Dies ist nicht gemeint im Sinne von Beliebigkeit und wird - hoffentlich - nicht missverstanden als Belanglosigkeit, sondern verbindet sich mit der Hoffnung, dass in der doppelten Interaktion des Lesers mit dem Text und darüber hinaus mit der Wirklichkeit, in der er lebt, eine Kaskade von Möglichkeiten freigesetzt wird. Möglichkeiten, die in der Realität bereits angelegt und neu rekombiniert in der Zukunft zu anderen Konsequenzen/Ergebnissen führen könnten, als in der Erzählung bereits schwarz auf weiß zu lesen ist.

Wie sich Generationen über ihre Lebensläufe hinweg miteinander verständigen, ist auch Thema von Kluges Frankfurter Poetikvorlesung. Er bezieht sich darin auf die 2012 erschienenen Neuen Lebensläufe, die den Ansatz des Vorbilds von 1962 erweitern. Wie Kluge ausführt, müssen Lebensläufe unter stark veränderten gesellschaftlichen und historischen Bedingungen im 21. Jahrhundert anders geschrieben werden als in seiner ersten Publikation, »weil die Erzählung von Lebensläufen sich verändert, wenn die Zeiten sich verändern «. ${ }^{38}$ Die Neuen Lebensläufe zeichnen sich denn auch dadurch aus, dass Kluge sich in ihnen u.a. auf die eigene Familiengeschichte bezieht, die bis ins 18 . Jahrhundert zurückreicht. ${ }^{39}$ Das rein heteroreferenzielle Erzählen wird, zumindest in den entsprechenden Passagen, selbstreferenziell. ${ }^{40}$ Diese Tendenz

schaft - Kognitionstechnik. Eine Skizze aktueller Perspektiven, 2. Aufl., übers. aus d. Engl. von Wolfram Karl Köck, Frankfurt a.M.: Suhrkamp 1990, S. 121.

So erläutert Fischer-Lichte, dass die rationale Erklärbarkeit von Emergenz nicht bedeute, dass man sie sich auch verfügbar machen könne: »Es sind heute gerade die Wissenschaften wie etwa die Chaosforschung und Mikrobiologie, die immer klarer die Erkenntnis hervortreten lassen, daß die Welt in diesem Sinne sverzaubert ist und sich dem sie zurichtenden Zugriff von Wissenschaft und Technologie - höchstwahrscheinlich zum Vorteil des Menschen - immer wieder entziehen wird, ähnlich unverfügbar wie jene autopoietische feedback-Schleife, die in Aufführungen wirkt.«Fischer-Lichte: Ästhetik des Performativen, S. 361.

38 A. Kluge: Theorie der Erzählung, DVD I, 5. Juni 2012, Kap. 3 »Neue Lebensläufe«, 26:58.

39 Vgl. Alexander Kluge: Das fünfte Buch. Neue Lebensläufe. 402 Geschichten, Berlin: Suhrkamp 2012, S. $115 \mathrm{f}$.

40 Besonders explizit wird diese Tendenz in den Episoden des ersten Abschnitts Die Lebensläufer und ihre Lebensgeschichten, darunter Stärkung mit zeitversetzter Wir- 
gipfelt im Kapitel »Ich« des mit »Chronik des Zusammenhangs« untertitelten Bandes Kongs große Stunde, wo Kluge auf die eigene Lebensgeschichte in Form der Simulation autobiografischen Schreibens Bezug nimmt.

Aufschlussreich ist dabei ein Gespräch mit dem Hirnforscher Wolf Singer bei dem klar wird, dass im Gehirn kein Konvergenzzentrum als Sitz des Ichs existiert. ${ }^{41}$ Vielmehr ist das bewusste Ich eine Art globaler Zustand, der durch eine große Zahl hoch verteilter neuronaler Prozesse vom Gehirn in Interaktion mit seiner Umwelt erzeugt wird. Es ist als Einzelphänomen nirgendwo lokalisierbar, sondern wird vom gesamten Gehirn als emergentes Phänomen hervorgebracht. Gibt es in einem essenziellen Sinn dieses Ich, von dem das autobiografische Schreiben immer ausgegangen war, in Wirklichkeit also gar nicht, so resultiert daraus, dass das erlebende und erzählende Ich, so wie es in der autobiografischen Erzählung auftritt, weitgehend als sprachliche und tendenziell sogar fiktionale Konstruktion begriffen werden muss. ${ }^{42}$ In genau diesem Sinn versteht sich der Begriff der Simulation im vorliegenden Kontext. Der Text nähert sich dabei auch in formaler Hinsicht der technisch emulierten Simulation an. So finden sich am Ende der Kapitel jeweils Verweise in Form von Lese- und Filmlinks, die ihn wie einen Hypertext mit weiteren Einträgen aus Kluges Produktion verbinden..$^{43}$

Das Ich resp. das Bewusstsein entzieht sich folglich in einem radikalen Sinn der Beschreibbarkeit und ist genau aus diesem Grund ein fundamental komplexes Phänomen. Macht man es zum Gegenstand einer Erzählung oder wissenschaftlichen Untersuchung ist zugleich gewiss, dass man es verfehlen wird oder nicht damit nicht zu Ende kommt bzw. ganz neue Denkansätze entwickeln muss und woanders landet als geplant. So bleibt selbst aus der Perspektive des erlebenden Ichs anstelle von Identität Differenz übrig, die in Form transitorischen Wechsels sogar die Innerlichkeit des Subjekts dominiert. Dieser Eindruck herrscht auch bei der Lektüre der genannten Texte von Kluge vor. Das damit aus der Innenperspektive verbundene Erleben be-

kung, Wie Zorn sich wandelt, Eine Kette von Vorfahren, Mein Urgroßvater mütterlicherseits sowie weiteren im Anschluss bis hin zu Wie ich Thomas Manns Villa umschlich. Vgl. ebd., S. 27-151.

41 Vgl. Alexander Kluge: Kongs große Stunde. Chronik des Zusammenhangs, Berlin: Suhrkamp 2015, S. $527 \mathrm{f}$.

42 Vgl. dazu das Kapitel »4.4 Konstruktionen«, in: Martina Wagner-Egelhaaf, Autobiographie, 2. aktual. und erweit. Aufl., Stuttgart: Metzler 2005, S. 60-65.

43 A. Kluge: Kongs große Stunde, S. 563. 
schreibt er in dem folgenden kleinen Abschnitt, der mit dem Titel Mein sogenanntes Bewußtsein überschrieben ist

»Ich schließe die Augen, konzentriere mich darauf, was ich INNEN sehe. Was ist das für ein Raum dort? Ich kann dort nichts ssehen`, höre nichts. Gerade, daß ich das AUSSEN ausgeschlossen habe. Wenn ich mich konzentriere, kann ich das, was ich um mich herum höre, wenn ich mit geschlossenen Augen dasitze, ausgrenzen. Es stürmen Bilder auf mich ein, ich sehe sie nicht in dem Dunkel, das ich INNEN nenne, sie sind auch nicht draußen, stehen nicht vor Augen. Sie sind nirgends GEGENWÄRTIG. Gewaltig rauschen die Assoziationen, die Selbsttätigkeit dessen, was - das ist eine Vermutung meine Hirnareale veranstalten [Herv. i.O.]. « $^{44}$

An dieser Beschreibung des auf sich selbst konzentrierten Bewusstseins fällt auf, dass es ihm gelingt, eine Differenz sowohl zwischen dem Innen und Außen einzuführen, als auch zu dem, was ihm auf der inneren Bühne begegnet. Der dabei entstehende, überwiegende Eindruck ist somit der des NichtIdentischen, bei dem das Bewusstsein nur an der Grenze-zu entsteht. Selbst die Dinge, die ihm in seiner Innerlichkeit begegnen, die Rede ist von »Bildern « und »Assoziationen«, sind als solche unterscheidbar von der Instanz, die diese Unterscheidung trifft, die jedoch dabei als solche nicht weiter analysierbar ist, es sei denn als Person des Autors AK identifiziert werden kann. ${ }^{45}$

Kommt man von hier aus auf das für alle komplexen Phänomene, somit auch auch für das komplexe Erzählen, charakteristische Fehlen einer zentralen Instanz/Funktionsstelle zurück, von dem bereits im ersten Kapitel die Rede war, so bestätigt Kluges Beschreibung der inneren Wahrnehmung und des daran gekoppelten Erlebens diesen Befund recht genau. Dass dabei das Ich, das, essenzialistisch verstanden, zuvor noch als zentrale Instanz in Frage gekommen wäre, nun aufgelöst wird in ein Spiel von Differenzen und eine Selbsttätigkeit, der das Bewusstsein beiwohnt, ohne die Ursachen dafür in den Blick zu bekommen, veranlasst Kluge, sich in dem vorhergehenden $\mathrm{Ab}$ schnitt Durch Armut reich: das Ich mit diesem Verschwinden autonomer Selbstgewissheit hilfesuchend an den Hirnforscher zu wenden:

\footnotetext{
44 Ebd., S. 528.

45 Zur Kritik dieser Art von Nicht-Identität als Widerstandsmoment gegen Formen totalitärer Simulation (Baudrillard) vgl. Michaela Holdenried: Autobiographie, Stuttgart: Reclam 2000, S. 54.
} 
»Was soll dann, fragte ich Wolf Singer, MEIN ICH heißen, wenn doch dessen Elemente und die gewaltigen, inzwischen dem Zufall überantworteten Verwandten meiner Ich-Fragmente, die Dinge, mir kaum noch gehören? Oft sind es Fremde, die ich nicht ganz verstehe, die mich stupsen. Denn meine Diamantschleifmaschine, die Härtestes und Loses stündlich zu einer Kette schmiedet, die, sobald man sie sich um den Hals legt, schon simperfekt $<$ meldet und zergeht, ist mein Eigentum, die Eigensinnigkeit meiner Milliarden Innerlichkeiten. Sie empfinden sich nicht als Vasallen, leben nicht wie Untertanen, aber sie gehören zu mir. Täten sie das nicht, wäre ich tot. Sagen Sie mir, lieber Herr Singer, was soll ich von einer solchen VERFASSUNG DES ICHS halten? [Herv. i.O.] $\ll^{46}$

Wenn der Hirnforscher darauf beipflichtend antwortet: »Sie sagen es, lieber Kollege, nur mit falschen Worten ${ }^{47}$ so ist das auf die Differenz zwischen den Diskursen von Wissenschaft und Erzählung zurückzuführen. Wenn die »Diamantschleifmaschine« des Erzählers übersetzt in die Sprache der Wissenschaft so etwas ergibt wie Bewusstseinsaktivität, so zeigt sich darin die größere Ausdrucksvielfalt, die über mehr Möglichkeiten der Kombination von Wort- und Bedeutungsfeldern verfügt, als es der Zwang zu Systematik und definitorischer Klarheit dem wissenschaftlichen Denken gestattet. Wenn man so will, ist damit von vornherein die Möglichkeit zum Erreichen höherer Stufen von Komplexität eher aufseiten des Erzählens als im wissenschaftlichen Diskurs gegeben, der von sich aus zur Reduktion tendiert, sobald seine Aussagen im Rahmen eines Geltungsbereichs den Charakter des Allgemeinen annehmen.

46 A. Kluge: Kongs große Stunde, S. $527 \mathrm{f}$.

47 Ebd., S. 528. 



\section{Erzählung und simulierte Wirklichkeit - Komplexes Erzählen in Bodo Morshäusers Berliner Simulation}

Es fragt sich nun, wie das Problem der Referenz literarischen Erzählens in jener künstlichen Hyperrealität zu denken ist, in der die Modelle der Simulation zum bestimmenden Schema dessen geworden sind, was als wirklich gilt. So spricht Baudrillard davon, dass die historisch überholten Dispositive der Repräsentation, d.h. des Verhältnisses der Zeichen zur Wirklichkeit, »als gespenstische, marionettenhafte Referenz ${ }^{1}$ in der nachfolgenden ZeichenOrdnung wiederkehren. Auf diese Weise verlieren Theoreme und Theorien wie die des natürlichen Wertgesetzes und der politischen Ökonomie des 19. Jahrhunderts ihre eigenständige Bedeutung als Erklärungsmodelle ökonomischer Realität, erscheinen aber durch ihre zentralen Begriffe, wie Gebrauchsund Tauschwert, als »imaginäres Referential ${ }^{2}$ in der nächst höheren Ordnung der Simulakren wieder. ${ }^{3}$ Innerhalb der Theorie von Komplexität lässt sich das nun auch so beschreiben, dass die revolutionären Veränderungen beim Übertritt von einer Zeichen-Ordnung zur nächsten sich durch Rekombination von Komponenten, die bereits auf der überkommenen Stufe angelegt waren, in einem avancierteren Zustand des gesellschaftlichen Systems realisieren. So verlieren einige dieser Komponenten, wie am Beispiel der Begriffe aus der politischen Ökonomie erläutert, ihre alte Bedeutung, erhalten dafür aber, wenn sie nicht ganz verschwinden, im neuen historischen Kontext einen veränderten Sinn.

\footnotetext{
1 J. Baudrillard: Der symbolische Tausch, S. 9.

2 Ebd.

3 Diese Übernahme ist bis hin zur gegenwärtigen Ordnung des Kodes (Simulakrum 3. Ordnung, vgl. ebd., S. 79) zu verfolgen, wo die Bezüge im Sinn einer »Simulationsreferenz« (ebd., S. 9), wieder erscheinen.
} 
In vergleichbarer Weise kann man nun auch für die Literatur zeigen, dass mit dem Aufkommen der Neuen Medien die überkommene GattungsEinteilung zugunsten intermedialer Formate sich in Auflösung begriffen zeigt. So tauchen der Roman und auch das Drama der ausgehenden Moderne als Hörspiel oder Hörbuch im Rundfunk oder als CD bzw. Stream recycelt wieder auf, während der zeitgenössische postmoderne Roman unter dem Druck einer durch Digitalisierung und globalisierte Ereignisverläufe rasant veränderten Wirklichkeit mit immer neuen Formexperimenten aufwartet, von denen einige in vorliegender Arbeit besprochen sind. Typisch ist dabei auch hier, dass die traditionellen Gattungsschemata, gewissermaßen analog zu ihrer Simulation in der jeweils neuesten Generation von Medien, auch im neueren Erzählen aufgelöst und neu zusammengesetzt werden. Das kann, wie in Bodo Morshäusers Erzählung Die Berliner Simulation, so weit gehen, dass selbst das literarische Erzählen nurmehr als simulatives begriffen wird, weil die Wirklichkeit als ganze aufgrund stetig zunehmender Komplexität längst nicht mehr, zumindest nicht auf herkömmliche Weise, erzählbar ist. An ihre Stelle sind Modelle der Simulation, also Strategien der Reduktion von Komplexität, getreten, die darüber entscheiden, was als wirklich gilt und was nicht.

So ist das Buch eingeteilt in zwei Abschnitte, die, angelehnt an das Prinzip kybernetischer Rückkopplung, mit Input und Output überschrieben sind. Während es im ersten Teil (Input) zu einer ersten flüchtigen Begegnung mit der aus London stammenden Wahlberlinerin Sarah, genannt Sally, kommt, die in einem Berliner Nachtcafé ihren Ausgang nimmt, schildert der zweite Teil (Output) die sich entwickelnde Bekanntschaft zwischen dem namenlosen Ich-Erzähler und der Fremden vor dem Hintergrund der Berliner Hausbesetzer-Szene Anfang der 8oer Jahre. Fungieren die Wahrnehmungen, Gedanken und Gefühle des erzählenden Ichs als Ausgangspunkt der Schreibbewegung, so kann ein solcher Ansatz als Probe aufs Exempel dafür verstanden werden, ob der Versuch, eine sich unter Bedingungen der mediatisierten Gesellschaft mehr und mehr entziehende Wirklichkeit im Erzählen doch noch einzuholen, überhaupt gelingen kann. Dafür steht als Synekdoche die Figur der Sally, die sich ihm am Ende des ersten Teils wieder entzieht, bevor es zu einer Wiederbegegnung im Zoologischen Garten zu Beginn von Teil Zwei kommt. Die Dynamik des Erzählens generiert dabei gewissermaßen zwei Attraktoren, die sich im erotischen Begehren nach Sally und zugleich in dem Verlangen manifestieren, einen adäquaten erzähleri- 
schen Ausdruck für den Kosmos der Berliner Realität zu Beginn der 8oer Jahre zu finden.

In welchem Maß dabei die Wirklichkeit bereits durch Simulation ersetzt ist, wird dem Erzähler klar, als er nach der Rückkehr von einer Kundgebung mit Polizeieinsätzen in der Kreuzberger Bergmannstraße den Fernseher einschaltet:

»Und dann, Sally, gehe ich nach Hause und was sehe ich? Ich sehe das Modell eines Fernsehbeitrags über das Modell einer Demonstration. Was wirklich ist, rutscht, wie üblich, hinten weg [...]. Nicht die Ereignisse, sondern die Modelle werden wiederholt. Längst dominieren sie, was passiert. Die Emotionen sind festgelegt auf jeweils zwei Möglichkeiten. In diesen Modellen sollen wir bleiben wie in einem Hamsterrad, denn in ihnen bleibt nichts wirklich; nur die Simulation. ${ }^{4}$

Wovon der Erzähler hier spricht, ist, mit Baudrillard gesprochen, die Erfahrung, dass die mediale Wiederholung der Wirklichkeit ihre unmittelbare, sozusagen ungefilterte Wahrnehmung substituiert hat. Was an ihre Stelle tritt, sind künstliche, elektronisch generierte Bilder, die das Bewusstsein infiltrieren und die Wahrnehmung steuern. In der aufgeregt-ruhelosen Erzählweise des Textes spiegeln sich sowohl das technisch bedingte >Flackern< dieser medialen Bilder wie die real vorhandene politische Unruhe in der Stadt, während Gegenwart, Vergangenheit und Zukunft (als leerer, unvorhersehbarer Horizont des Jetzt) aus der subjektiven Perspektive des erzählenden Ichs oszillierend ineinander gleiten. Äußere Bezugspunkte bilden dabei neben den Begegnungen mit Sally und ihren Kumpanen, die Straßenkämpfe der Hausbesetzer und ihrer Sympathisanten mit der Polizei sowie Ereignisse der Berliner Zeitgeschichte. ${ }^{5}$ All dies vermischt sich mit privaten Erlebnissen aus der Erinnerung des Erzählers, der in Berlin aufgewachsen ist und Sally, als Adressatin seiner Narration, vor den einschlägigen zeitgeschichtlichen Hintergründen gewissermaßen mit seiner Welt vertraut macht. Dieses Szenario verdichtet sich im Verlauf des Textes, nachdem die Gruppe um Lora, Sally,

4 B. Morshäuser: Berliner Simulation, S. 78.

5 Entfaltet wird ein ganzes Panorama zeitgeschichtlicher Ereignisse vom Arbeiteraufstand 1953, dem Kennedybesuch zehn Jahre später, der Konfrontation der Schutzmächte, die sich 1961 am Checkpoint in der Friedrichstraße mit Panzern gegenüberstehen, bis hin zu den einschüchternden Tiefflugmanövern sowjetischer Jagdflugzeuge über der Potsdamer Straße 1964 und vieles mehr. Vgl. ebd., S. 79-86. 
Terry und Martin, zu der, sozusagen als ihr Chronist, auch der Erzähler gehört, mit einer amerikanischen Limousine am Hohenzollerndamm zufällig in Filmdreharbeiten geraten. Durch das Erzählen werden sie ungeplant zum Teil des filmischen Plots, auch wenn es nach Verhandlungen mit dem Regisseur nicht zu einem Auftritt vor laufender Kamera kommt. ${ }^{6}$

Spätestens hier wird offenkundig, dass Film bzw. mediale Realität und Wirklichkeit sich im Bewusstsein des Erzählers durchdringen und auf diese Weise ununterscheidbar werden. Ereignisse aus der Vergangenheit, wie das Attentat auf Rudi Dutschke, eine Begegnung mit Ella Fitzgerald oder die Vietnam-Demonstrationen während der Studentenbewegung, ${ }^{7}$ werden aus der Perspektive unmittelbarer Teilnahme vergegenwärtigt und durch den Akt der Verschriftlichung in den Ereignishorizont des gelebten Augenblicks hineingezogen. Dabei ist die Wirklichkeitserfahrung des erzählenden Ichs die einer sich endlos fortsetzenden Gegenwart autobiografischer Wahrnehmung (Scheffer), in welcher die Erinnerung an die historischen Ereignisse aus der Berliner Vergangenheit mit dem instantan begegnenden Geschehen interagiert, d.h. sich mit ihm rückkoppelt. Der Input augenblicklicher Wahrnehmung triggert dabei die Gedächtnisinhalte, die als reaktualisierte das Gegenwartsbewusstsein des Erzählers erweitern und sich somit als Output (im Sinn emergenten Hervorbringens) verstehen lassen. Das damit verbundene Zeitgefühl drückt sich für den Erzähler in einer seltsamen Verzögerung aus, die das zeitliche Geschehen von ihm abrückt: »Aber ich weiß, daß ich besetzt bin von einer Gegenwart, die noch nicht eingetroffen ist. ${ }^{8}$ Das, was geschieht, geschieht folglich nicht unmittelbar, sondern mit einem zeitlichen Verzug, der darauf verweist, dass sich die mit der Gegenwart verknüpften Hoffnungen, Erwartungen und Ängste noch nicht erfüllt haben, vielmehr erst dabei sind, sich zu realisieren. Eben gerade daraus ergibt sich ein Spielraum der Reflexion, der es dem Erzähler erlaubt, auf kritische Distanz zu den medialen Simulationen der Realität zu gehen, wobei fraglich bleibt, ob dies letztlich ausreicht, um die Simulation zu durchbrechen.

Der Text, der diesen Prozess in Form der literarischen Erzählung darstellt, wird nun zugleich lesbar als Protokoll der Transgression einer überkommenen Vorstellung von Geschichtsschreibung, die Geschichte Geschichte sein

\footnotetext{
6 Vgl. ebd., S. $100 \mathrm{f}$.

7 Vgl. ebd., S. 104-107.

8 Ebd., S. 25.
} 
lässt, also vergangen, und deren Geschehen deswegen nur in den sprachlichen Zeitformen des Präteritums und des Plusquamperfekts adressierbar ist. Zwar ließe sich prima facie davon sprechen, dass doch Geschichte, wenn auch auf subjektiv gebrochene Weise, im Text durchaus rekonstruiert wird, indem der Erzähler eine Koinzidenz von Ereignissen mit zeitlichen Daten (Jahreszahlen) herstellt. Dabei bliebe jedoch die Selektion und Rekombination der Geschehnisse durch das erzählende Bewusstsein unberücksichtigt. Sofern es dem Autor darum geht, die strukturellen Bedingungen des neuen Paradigmas der Simulation (Simulakrum 3. Ordnung), deren Konsequenzen für die Wahrnehmung von Wirklichkeit sowie ihrer literarischen Erzählbarkeit einsichtig zu machen, bekommt das historische Zitat aber nun eine andere Bedeutung. Es steht nicht mehr für das Ereignis an sich, sondern für die Rekombinierbarkeit der in ihm entworfenen Welt bzw. Weltsicht mit dem Input gegenwärtiger Wahrnehmungsinhalte, welche gemeinsam den Output des Erzählakts erzeugen, in den beide Momente mit eingegangen sind. Geschichte und Gegenwart fallen also zusammen, nicht auseinander, und bilden im Bewusstsein des erzählenden Ichs eine einzige, wenngleich von medial bedingten Diskontinuitäten der Wahrnehmung durchzogene Wirklichkeit.

Was nunmehr als gespenstische, marionettenhafte Referenz im Sinne Baudrillards innerhalb des durch mediale Wirkungen transformierten Wahrnehmungsbewusstseins von neuem wiederkehrt, wäre die traditionelle, sukzessiv-lineare Form literarischen Erzählens ebenso wie die analoge Darstellungsweise von Geschichtsschreibung, mit denen der Text von Morshäuser bricht. Sofern diese historisch überkommenen Formen der Darstellung als Bedingung der Möglichkeit innovativer Schreibweisen erneut sichtbar werden, können sie neben den Erinnerungs- und Wahrnehmungsinhalten aus dem Erzählerbewusstsein, von denen naheliegt, dass sie aus dem autobiographischen Gedächtnis des empirischen Autors stammen, als Nullstelle des Diskurses identifiziert werden. ${ }^{9}$ Man ist hier unwillkürlich an

Auch wenn Morshäuser, indem er diesen, anders als Gustafsson und Kermani, namenlos belässt, eine bewusste Identifizierung des Autors mit dem Erzähler vermeidet, sind die Ähnlichkeiten, wenn nicht Übereinstimmungen zwischen beiden Identitäten kaum zu übersehen. So ist Morshäuser 1953 in Berlin geboren und dort auch aufgewachsen. Er hat folglich, wie der Erzähler, die 6oer Jahre, die als wichtigste Referenz den Text durchziehen, daselbst miterlebt und kennt darüber hinaus den Alltag der Metropole sehr genau, was wiederum den authentischen Charakter der Beschreibungen von Drogenszene, Autostrich und Straßenkampf in der Berliner Simulation erklärt. Wohl 
Lars Gustafssons Verfahren erinnert, den literarischen Text mittels einer seltsamen Schleife mit sich selbst rückzukoppeln, um dadurch als neuen Output eine erweiterte Ebene der Wahrnehmung und des Denkens zu generieren. Die damit verbundene Mise en abyme heißt auf der einen Seite Bedeutungsverlust der überkommenen linearen Erzählweise. Andererseits aber wird eine neue Möglichkeit der Beschreibung/Erzählung von Wirklichkeit erkennbar. Die neue Form mag der beschriebenen/erzählten Welt immer noch ähneln, jedoch nicht mehr im Sinn einer Kopie, sondern der Strukturanalyse, in der zutage tritt, nach welchen Regeln die erzählte Wirklichkeit funktioniert, nämlich denen der technisch realisierten Simulation von Realität.

Noch etwas zeichnet sich an dieser Stelle ab. Das ist der Umstand, dass nicht nur angesichts einer durch Simulation modellierten Wahrnehmung von Wirklichkeit, sondern auch durch deren mediale Erweiterungen in Richtung Virtual Reality, Computer-Gaming, webbasierten sozialen Netzwerken, Online-Dating usw., das, was wirklich, d.h. außerhalb davon, geschieht, mehr und mehr an Interesse verliert, weil es ohne Aufmerksamkeit verbleibt. ${ }^{10}$ Wenn zuvor, etwa im Zusammenhang der Analyse von Gustafssons Poetik, von Sinnentzug und Nicht-wahrgenommen-Werden die Rede war, so ist im Ansatz damit genau dieses Phänomen der Überformung des Sensoriums durch mediale Simulationen gemeint, das den dafür sensibilisierten Autoren mehr und mehr bewusst wurde und wird. Alles, was noch nicht den Modellierungen der Simulation gemäß ist und damit den herrschenden Wahrnehmungsschemata entspricht, fällt unter den Tisch, weil es gar nicht

nicht zufällig ist Berlin auch der Schauplatz weiterer Erzählungen wie Blende (1985), Nervöse Leser (1987) und Der weiße Wannsee (1993).

Anders verhält es sich für den Fall, dass schwerwiegende Konsistenzprobleme zwischen einem in der Wirklichkeit wahrgenommen Geschehen und seiner (oder einer stellvertretenden) Darstellung in den Medien auftreten. So wäre der Tod eines Jungen durch einen Busunfall (vgl. B. Morshäuser: Berliner Simulation, S. 80-82 und 99) unter anderen Umständen bestenfalls in der Kategorie Vermischtes der Lokalzeitungen erwähnt worden. Durch den Kontext der polizeilichen Räumung von besetzten Häusern wird jedoch sofort ein Zusammenhang zu den massiven Polizeieinsätzen bzw. zu staatlicher Cewalt generell hergestellt, d.h. damit in ursächlichen Zusammenhang gebracht, und in den Medien mit großer Wirkung verbreitet. Der Text nimmt hier Bezug auf den Tod des 18-jährigen Hausbesetzers Klaus-Jürgen Rattay, der am 22. September 1981 nach der Räumung von besetzten Häusern durch die Polizei an der Kreuzung Bülowstraße/Potsdamer Straße von einem Bus erfasst und zu Tode geschleift wurde. Vgl. dazu https://de.wikipedia.org/wiki/Klaus-J\%C3\%BCrgen_Rattay vom 30.10.2020. 
mehr in die Wahrnehmung gerät, die in den Schleifen der medialen Wiederholung von Wirklichkeit verblendet um sich selbst zu kreisen beginnt. Ein Effekt, den der Erzähler auch als »Wiederholungsfalle «" ${ }^{11}$ beschreibt. Sobald Wirklichkeit zunehmend auf die Form medialer Formate reduzierbar ist, wird sie in einer Weise manipulierbar, die alternative Möglichkeiten der Interpretation weitgehend ausschließt. Es steht sozusagen schon vorher fest, wie die Dinge zu verstehen sind. Die Medienkonsumenten haben nicht darüber zu entscheiden, sondern die geltende Version einfach nur zur Kenntnis zu nehmen. So konstatiert der Erzähler angesichts der Berichterstattung im Fernsehen:

»Ich mag es nicht mehr sehen. Der Kurzbeitrag über die Demonstration wiederholt nichts weiter als einen Kurzbeitrag über eine Demonstration, das Fernsehen macht, wie üblich, Fernsehen. Ich mag es nicht mehr sehen, und doch schau ich hin. Der Anlaß wird verschwiegen, die Teilnehmerzahl ist getürkt, die Frage, wer die Gewalt begann, war längst vorher geklärt. $\ll^{12}$

Hier wird nicht nur durch die redundante Formulierung erkennbar, sondern auch inhaltlich explizit gemacht, auf welche Weise Wirklichkeit mehr und mehr als medial inszenierte erlebt wird.

Neben der medialen Strategie zur Herstellung von Normalität (Kontrolle des Geschehens) ist daran zudem eine Sehnsucht nach Authentizität ablesbar, die sich durch den ganzen Text zieht. Die Suche des Erzählers nach dem Authentischen in seinem eigenen Leben und in den Beziehungen zu den anderen Figuren, die sich über ihre Qualität als Zufallsbekanntschaften hinaus darin als wesentlich erweisen, dass man sich nichts vormacht, sondern die Dinge beim Namen nennt, kann als Gegenbewegung zur Mediatisierung der Gesellschaft und der damit verbundenen Simulation der Realität verstanden werden. Die daraus resultierende, an sich unentscheidbare Frage, ob nun die subjektiv erlebte Wirklichkeit als Geschichte des eigenen Lebens mit den daran sich knüpfenden Erinnerungen im Sinne endlos autobiografischer Wahrnehmung oder die durch mediale Simulation modellierte Wirklichkeitswahrnehmung wirklicher sei, führt am Ende zu einer überraschenden Wendung in der Erzähldynamik. Ähnlich wie im Roman Buenos Aires. Anderswelt kommt es auch in der Berliner Simulation zur Verschmelzung ontologisch eigentlich getrennter Bezugsebenen. Dabei interagieren die medialen Bilder aus der 
Vergangenheit mit der Wahrnehmung des gegenwärtigen Geschehens. Das Szenario der Studentenrevolte in der zweiten Hälfte der 6oer Jahre tauscht sich im Bewusstsein des Erzählers aufgrund vieler Übereinstimmungen mit den Straßenkämpfen der Hausbesetzer in der erzählten Gegenwart des Jahres 1981 aus. Die medialen Bilder der 68er Revolte, die in der Erinnerung des Erzählers immer noch lebendig sind, gehen dabei in die gegenwärtige Realität über, die als genauso entregelt erlebt wird wie eineinhalb Jahrzehnte zuvor.

So fallen Vergangenheit und Gegenwart zusammen in einen gemeinsamen Horizont des Versuchs der Veränderung gesellschaftlicher Verhältnisse, die sich als veränderungsresistent erwiesen haben. Daher erscheinen auch die Handlungssequenzen am Schluss der Erzählung wie Zitate aus der Zeit der Studentenproteste. Wenn dort von einer Vietnamdemonstration, der »Abschleppveranstaltung « ${ }^{13}$ parkender Autos am Kurfürstendamm sowie einem Kaufhausbesuch die Rede ist, bei der die Sprinkleranlage ausgelöst wird, ${ }^{14}$ so sind dies Anspielungen auf zentrale, politisch bedeutsame Ereignisse dieser Zeit, ${ }^{15}$ die aber in der realen Gegenwart ohne weitergehende Bedeutung bleiben und darum wie Versatzstücke wirken. Auf diese Weise erklärt sich auch der slapstickartige, ironisch augenzwinkernde Stil, der durch uneigentliche Redeweise die Wiederholung der ikonisch gewordenen Ereignisse evozieren möchte und zugleich parodistisch hintertreibt. Wenn die Beschreibung eines Molotow-Cocktails, den Terry mit sich führt, als »Flasche [...] [m]it einem Stück Stoff darin ${ }^{16}{ }^{16}$ wie auch die Kaufhausszene selbst, im Sinne eines parodistischen Verweises auf die historisch gewordenen Frankfurter Kaufhausbrände erscheint, so wird damit zugleich gesagt, dass die im Rahmen des militanten Kampfes gegen die gesellschaftliche Ordnung von der RAF verübten Anschläge ihre substanzielle Bedeutsamkeit als Strategie für die Veränderung von Gesellschaft längst eingebüßt haben.

Auf diese Weise kann das Erscheinen des Protest-Szenarios der 6oer Jahre in der bereits durch Simulation geprägten Gegenwartswahrnehmung des Erzählers als gespenstische, marionettenhafte Referenz im Sinne Baudrillards

\footnotetext{
13 Ebd., S. 107.

14 Vgl. ebd.

15 Vor allem gemeint sind die seit 1966 in West-Berlin stattfindenden Anti-VietnamDemonstrationen und die Brandanschläge der RAF auf zwei Frankfurter Kaufhäuser im April 1968.

16 B. Morshäuser: Berliner Simulation, S. 108.
} 
verstanden werden. Dieser Effekt wird jedoch nicht länger als Schock der Entwirklichung erlebt, der mit dem Verlust authentischer Identität und der Unmöglichkeit überhaupt noch selbstbestimmt in Kontakt zur Wirklichkeit und zu sich selbst treten zu können, wie bei Herbst, sondern als Bedingung zeitgenössischer Weltwahrnehmung und deren Erzählbarkeit anerkannt. Entsprechend ist der Duktus des Erzählens parodistisch, weil sich Situationen, Konflikte und der politische Umgang damit wiederholen, als sei seit den AntiVietnam-Demonstrationen und den Todesschüssen auf Rudi Dutschke und Benno Ohnesorg nichts geschehen. Das Erzählen weiß also nicht anders damit umzugehen, als die aus dem Gedächtnis des Erzählers stammenden zeitgeschichtlichen Inhalte als transparente Folie der Darstellung zu wiederholen und dabei in eine komische Richtung umzulenken, was exakt der klassischen Definition der literarischen Parodie entspricht, wie man sie etwa bei Scaliger findet. ${ }^{17}$ Die Motivation dafür ist jedoch in diesem Fall nicht einfach in dem komischen Effekt als solchem oder der unernsten Kritik an Autor oder Text zu sehen, auf welche sich die Parodie bezieht, sondern vielmehr in der Ratlosigkeit angesichts der Deutungshoheit allgegenwärtiger Simulation, die einem keine Wahl lässt, als sich die eigene Ohnmacht durch eine eher unfreiwillig produzierte Komik einzugestehen. Als Ziel der parodistischen Intention kann somit neben der ubiquitären medialen Simulation das herkömmliche lineare Erzählen angesehen werden, das angesichts der in Echtzeit auftretenden Simulakren scheitern muss, ohne dass, anders als bei Kermani, eine adäquate narrative Antwort schon gefunden wäre. Das gespenstische oder marionettenhafte Wiedererscheinen der genannten Referenz(en) und der literarische Umgang damit ist darum im gegebenen Kontext eher als Kompensation ungelöster gesellschaftlicher Widersprüche und der daraus resultierenden Konflikte zu verstehen, die sich im Bewusstsein des Erzählers wie in einer Endlosschleife wiederholen, während eine definitive Lösung dafür auf Dauer ausbleibt.

Somit kann auch die zuvor vom Personal der Erzählung aufgeworfene Frage, ob es sich nicht umgekehrt bei den in der erzählten Gegenwart stattfindenden Ereignissen um geschichtsträchtige Vorgänge handelt, ${ }^{18}$ durch die Prozessdynamik eines Erzählens beantwortet werden, dem angesichts der verwirrend komplexen Gegenwart nichts anderes übrig bleibt, als Referenzen

17 Vgl. G. Genette: Palimpseste, S. 26.

18 Vgl. B. Morshäuser: Berliner Simulation, S. 85 f. und 99. 
zu einer Vergangenheit herzustellen, die sich als überlebt erweist. Daher entpuppt sich auch die Frage danach, was es ist, das ein Ereignis bzw. Handeln von Personen in der Gegenwart nachträglich historisch bedeutsam werden lässt, vor dem Horizont einer durch Simulation modellierten Wahrnehmung schließlich als Scheinfrage, die den Erzähler zu der lakonischen Feststellung veranlasst: »Längst handelt es sich um Figuren auf dem weiten, alltäglichen Feld der Simulation. ${ }^{19}$ Daran vermag selbst der tragische Todesfall eines Jungen, real gemeint ist hier der 18-jährige Hausbesetzter Klaus-Jürgen Rattay, der am Vortag im Umkreis der Straßenkämpfe von einem Omnibus überfahren worden war, nichts zu ändern. ${ }^{20}$ Die ubiquitäre Logik der "Schaltkreise und Fangnetze« medialer Simulation löscht den Topos vom »Ort der Geschichte ${ }^{21}$ und die ihm zugrundeliegende Semantik des Zentrums, mit dem sich (historische) Bedeutung zuvor noch verband. ${ }^{22}$

\footnotetext{
19 Ebd., S. $85 \mathrm{f}$.

20 Vgl. ebd., S. 99. Zu den zeitgeschichtlichen Hintergründen vgl. auch FN 10 des laufenden Kapitels.

21 B. Morshäuser: Berliner Simulation, S. 99.

22 Vgl. ebd.
} 


\section{Genese von literarischer Bedeutung durch strukturelle Selbstähnlichkeit}

Dekonstruiert werden auf diese Weise Bedeutungen (bzw. semantische Werte), die in modalen Kontexten der Linearität (Schrift) oder der Fläche (Bild) eingebettet sind, zugunsten eines vieldimensionalen, fraktal gebrochenen Raums, in dem sich das, was zuvor als Referenzbeziehung zwischen Zeichen und Bezeichnetem eingeschrieben war, nun in Form der Dynamik emergenter Prozesshaftigkeit entfaltet. Dabei erweisen sich die imaginären Figurationen einer erzählten Welt, die vom Bewusstsein des Lesers in simultaner Interaktion mit dem literarischen Text und der subjektiven Wirklichkeit hervorgebracht werden, durch Strukturen der Selbstähnlichkeit - wie in der fraktalen Geometrie - als mit diesen Bezugssystemen und deren Komponenten rückgekoppelte. Auf diese Weise wird Bedeutung nicht mehr im Sinn einer passiven Abbildung von Merkmalen der Außenwelt verstanden, sondern, wie Francisco Varela schreibt, durch Interaktion des Gehirns mit seiner Umwelt erzeugt. ${ }^{1}$ Das, was dabei als Bedeutung emergiert, ergibt sich, systemtheoretisch gesprochen, als kohärenter Attraktor, der durch übergreifende neuronale Muster vor dem Hintergrund inkohärenter Aktivität entsteht. $^{2}$ Bedeutung kann so als »kreative Dimensionierung [...] auf der Grundlage einer Lebensgeschichte ${ }^{3}$ verstanden werden, die, wie am Beispiel des Erzählers in Morshäusers Berliner Simulation gezeigt, auch für das literarische Erzählen von eminenter Bedeutung ist.

An dieser Stelle ist dem Einwand zu begegnen, dass doch das umfassendere Bezugssystem in dieser Triade, nämlich die subjektiv erlebte Wirklichkeit des Lesers, die beiden anderen Positionen als Elemente bereits enthält,

\footnotetext{
1 Vgl. F. Varela: Kognitionswissenschaft - Kognitionstechnik, S. 109.

2 Vgl. ebd.

3 Ebd.
} 
sodass die topologische Beziehung zwischen ihnen sich gar nicht als Interaktion beschreiben lässt, sondern als Enthalten-sein-in im Sinne mereologischer bzw. mengenmäßiger Definition. Somit wäre sowohl der literarische Text in dieser Wirklichkeit enthalten, also Teil von ihr, wie auch das erlebende Subjekt von (s)einer Wirklichkeit umgeben ist, die, sofern sie von anderen geteilt und nicht solipsistisch hypostasiert wird, dieses gewissermaßen als Bestandteil oder, mit mehr Handlungsorientierung gedacht, als Agent umgibt. Dabei würde jedoch unterschlagen, dass das wahrnehmende Subjekt seine Wirklichkeit durch Interaktion mit der objektiv vorhandenen Umwelt allererst hervorbringt. Der Eindruck, dass es in ihr enthalten sei, weil es von ihr umgeben ist, ebenso wie der Text ja immer nur in einer Umgebung vorkommt, geht darauf zurück, dass die Hintergrund-Prozesse des emergenten Hervorbringens von Wirklichkeit der naiven Beobachtung unzugänglich sind. Sobald diese in den Neuro- bzw. Kognitionswissenschaften der Betrachtung zugänglich gemacht werden, entpuppt sich jener Eindruck jedoch als Schein phänomenaler Wahrnehmung, der sich durch neuronale Aktivitätsmuster erklären lässt, die im Gehirn in netzwerkartig verteilter Form vorkommen. ${ }^{4}$

Logisch gesehen ist zudem mit diesem Eindruck eine Petitio principii verbunden, sofern das Zustandekommen der Wahrnehmung als das zu Erklärende (Explanandum) nicht als Ergebnis eines neuronalen HintergrundProzesses interpretiert, sondern im Sinne einer bloßen Abbildung (Repräsentation) einer bereits gegebenen Wirklichkeit verstanden wird. Der Prozess der Genese von Wahrnehmung resp. Wirklichkeit, der eigentlich erklärt werden soll, wäre somit bereits als Gegebenheit, nämlich als Eindruck des Umgeben-seins, in den Voraussetzungen/Prämissen (Explanans) enthalten. Um diesen Zirkelschluss zu vermeiden, wird das Paradigma der abbildenden Repräsentation durch die Kategorie kybernetischer Interaktion ersetzt, die über performative Input-Output-Rückkopplungen das Phänomen erst hervorbringt, das andernfalls zirkelartig als Voraussetzung und zugleich Ergebnis des unvermittelten Umgeben-seins (von Wirklichkeit) die Einsicht in neuere Formen des epistemischen Designs der Genese von WirklichkeitsWahrnehmung antinomisch verwehren würde.

In Hinsicht auf die Praxis der Lektüre führen diese Überlegungen des Weiteren zu der Frage, ob man als Leser, also während man liest, denn überhaupt mit der Wirklichkeit verbunden ist oder nicht vielmehr mit der Imagibringen von Wirklichkeit. Beispiele«, ebd., S. 97-109. 
nation einer solchen als erzählter Welt. In der Tat scheint es so zu sein, dass wir, indem wir den Text auf unserer mentalen Bühne zur Aufführung bringen, die umgebende Wirklichkeit sozusagen ausblenden. Und solange wir dies tun sind wir gewissermaßen, wie man sagt, aus der Welt, weil die Konzentration auf den Text als Vergegenwärtigung dessen, was in ihm zur Sprache gebracht wird, unsere ganze Aufmerksamkeit verlangt. Dennoch ist ein solches instantanes Verständnis von Wirklichkeit nicht genug, weil damit nicht all das zum Ausdruck gebracht werden kann, was dem Subjekt als wirklich gilt. So kommt es während der Rezeption nicht nur zur oberflächlichen Immersion mit der vom Text evozierten Welt, vielmehr werden auch explanatorisches Hintergrundwissen und vor allem die mit seiner Identität verbundenen Erfahrungen, weltanschaulichen Überzeugungen und sogar die mit früheren Stufen/Zuständen/Befindlichkeiten des eigenen Selbst verknüpften, qualitativen Wahrnehmungsinhalte aus der Erinnerung des lesenden Subjekts durch Interaktion mit der erzählten Wirklichkeit aufgerufen bzw. reaktualisiert. Es lässt sich daher sagen, dass die so verstandene Lektürepraxis nur bei oberflächlicher Betrachtungsweise als Weltflucht charakterisierbar wäre. In Wahrheit verhält es sich aber doch so, dass dem lesenden Subjekt sich erst dadurch Dimensionen von Wirklichkeit erschließen, die andernfalls, also ohne den Akt der inneren Aufführung des Textes und den damit verbundenen Prozessen der Reflexion, der Selbstprüfung bestehender Wissens- und Erfahrungsbestände mit der Möglichkeit ihrer anschließenden Revidierung, überhaupt nicht da wären oder nur rudimentär entwickelt blieben. ${ }^{5}$

Dies gilt freilich mindestens in gleichem Maße für die Seite expressiver Produktion. Auf welche Weise Selbstfindung und Selbsthervorbringung dabei in der »Figur der Selbstbeschreibung « zusammen gehen, präzisiert im Folgenden Emil Angehrn: »Auf der anderen Seite entdecken wir uns selbst im Ausdruck. Wir werden mit uns selbst bekannt, finden die eigene Stimme im Gespräch mit anderen. Wir lernen unsere Leidenschaften und Gefühle kennen, begegnen unseren Ängsten und Phantasien im Versuch, sie auszudrücken und differenziert zu beschreiben. Wir werden uns klarer über uns selbst, können uns im Ausdruck gleichzeitig hinterfragen, Vorurteile aufdecken, uns selbst korrigieren und uns um die Übereinstimmung mit uns bemühen. Der Ausdruck ist Arbeit an uns selbst, eine Tätigkeit des Durchleuchtens und Genauer-Sehens, möglicherweise auch des Zurückkommens und Zurechtrückens. Selbsterkenntnis auf den Wegen des Ausdrucks, auch den erst zu bahnenden, freizulegenden Wegen des Ausdrucks ist nicht nur ein Registrieren, sondern eine Selbstaufklärung und ein Mitsich-ins-Reine-Kommen - wenn auch nie gefeit vor der Gefahr des Sich-Täuschens, ja, des Sich-Verdeckens und Sich-Verstellens. «Emil Angehrn: Sein Leben schreiben. Wege der Erinnerung, Frankfurt a.M.: Klostermann 2017, S. 24. 



\section{Wende zum fraktalen Erzählen - Reinhard Jirgls Roman Abschied von den Feinden}

Hängen die Spielarten der komplexen Erzählung in den bisher behandelten Beispielen mit bestimmten Mustern auf der Ebene der Darstellung des erzählten Geschehens zusammen, so betreffen diese Besonderheiten der Struktur im Fall von Reinhard Jirgl zudem die basalere Ebene des alphanumerischen Kodes, aus dem sich seine Texte, verstanden im Sinn einer Matrix, zusammensetzen. Wohl nicht zufällig ist es, dass auch das Repertoire der Zeichen mit dem die meisten Programmiersprachen arbeiten alphanumerisch ist. ${ }^{1}$ Damit wäre also auch der Zeichensatz konnotiert, mit dem Programmierer Programmroutinen schreiben, die dann in den Binärkode der Maschinensprache übersetzt die digitale Simulation für den Benutzer, Virilios Terminal-Bürger, erzeugen. So nähert sich die Untersuchung abermals, scheint es, dem Szenario des Ich-Erzählers und Programmierers Alban Herbst an, mit dem Unterschied jedoch, dass die Benutzeroberfläche diesmal wieder ein analoger, deswegen nur bedingt als kybernetisch zu verstehender Text ist. Dennoch deutet dies tendenziell auf ein Jenseits der geschriebenen bzw. gedruckten Schrift im Universum der technisch erzeugten Bilder und damit der digitalen Simulation voraus. ${ }^{2}$

Damit wird abermals die Nähe zwischen literarischem Text und der Programmierung von Algorithmen evoziert. Das wiederum rückt die Möglichkeit in den Fokus, »Texte als Programme, Narrative als Algorithmen, Erzählungen als Berechnungen aufzufassen [Herv. i.O.]«, wie Sven Kosub schreibt. S. Kosub: »Textuelle Berechenbarkeit«, in: Koschorke (HC.), Komplexität und Einfachheit (2017), S. 240.

2 Vgl. dazu V. Flusser: Die Schrift, S. 23. Flusser spricht auch davon, dass die Schrift »durch geeignetere Codes« (ebd., S. 13) überholt werden könnte und dadurch die mit dem 
Jirgel hat in dem Essay Das poetische Vermögen des alphanumerischen Codes in der Prosa bis ins Einzelne die von der gewöhnlichen Orthografie abweichende Verwendung der beiden Konjunktionen »und« bzw. »oder« sowie der Numerale erläutert, die in seinen Texten, je nach Schreibweise, unterschiedliche Bedeutung haben. ${ }^{3}$ In diesem Zusammenhang weist der Autor auch darauf hin, dass »es sich keineswegs um eine starre festgezurrte Systematik« handle, sondern vielmehr der »unmittelbare Kontextbezug « ${ }^{4}$ für das Verständnis ausschlaggebend sei. Auch für die Interpunktion, besonders für die Setzung von Frage- und Ausrufezeichen, die bei Jirgl nicht am Ende, sondern am Anfang der entsprechenden Sätze stehen und gewissermaßen als "Notenschlüssel « ${ }^{5}$ wirken sollen, ist die Abbildung »des realen Sprech-Verhaltens von Menschen im Alltag ${ }^{6}{ }^{6}$ ausschlaggebend. Es geht ihm dabei in erster Linie darum, dass »es sich bei Textgebilden (auch) um die Darstellung von Sprach-Gesten und -Gebärden in deren körperlicher Entsprechung handelt [...]. « Er macht somit an vielen Stellen die sprachliche Konvention rückgängig und ersetzt sie durch einen alternativen, autorspezifischen Kode, der die bewusste Erweiterung der Kontextsensitivität des sprachlichen Materials zum Ziel hat. Dies

Schreiben verbundenen Formen des Lebens und des Bewusstseins als historische obsolet würden. Vgl. ebd., S. 7 f. und 13.

3 Die unterschiedlichen Schreibweisen stehen für zeitliche Aufeinanderfolge (und), Cleichzeitigkeit (u), Verflechtung von Cegensatzpaaren (u:), Indikation für Ceschäftliches (\&), Verwendung in Traumtexten ( + ) bzw. Variantenvergleich mit ungleicher (oder) und gleicher Wahrscheinlichkeit (od) sowie den Hinweis auf die wahrscheinlichere Variante (od:). Vgl. Reinhard Jirgl: „Das poetische Vermögen des alphanumerischen Codes in der Prosa«, in: Ders., Gewitterlicht. Erzählung, 5. Aufl., Hannover: Revonnah 2003, S. 50-77, hier S. 68 f. Ähnliches gilt für die Verwendung der Ziffern als Numerale (»1«) oder als ausgeschriebenes Wort (»ein«, »eine«, »eines«), wobei je nach Schreibweise entweder die Knappheit des zeitlichen Bezugs von Vorgängen oder im Cegenteil ihre Langsamkeit und Dauerhaftigkeit gemeint sind (vgl. ebd., 70-73). Weiterhin auch die Charakterisierung von Menschen und Dingen in ihrer optischen Erscheinung und ihrem Habitus als »hager«, »dürr«, »flink«, »beweglich« bzw. »fragil«, »kalt«, »spitz«, »hart« (ebd., S. 71), sowie in ihrer gegenteiligen Bedeutung als »wohl proportioniert«, »genussfreudig«, »gesellig«bzw. »robust, gedrungen«, »warm«, »rundlich«. Ebd., S. 72. Dabei sind die Bedeutungen, ihre Varianten und Oppositionen im Einzelnen noch weitaus differenzierter, als es hier zusammenfassend dargestellt werden kann.

4 Ebd., S. 70.

5 Ebd., S. 74.

6 Ebd., S. 73.

7 Ebd., S. 74. 
jedoch nicht als Selbstzweck, sondern mit der Absicht, die real gegebene Komplexität verbalen wie non-verbalen menschlichen Verhaltens, die in wesentlichen Hinsichten von der konventionellen Orthografie nicht erfasst wird, zu versprachlichen.

Diese phonetische Schreibweise verschiebt den Text in Richtung Lautmaterial und bedeutet zugleich eine »Entscheidung gegen den Vortrags- und für den Lese-Text. « ${ }^{8}$ Der Schritt vom Kode der orthografischen Norm zur Materialität der gesprochenen Sprache bringt an vielen Stellen eine Entregelung bzw. Neuanpassung mit sich, die es dem Autor gestattet, die Sprechweisen von Menschen, die regional spezifisch und zugleich Ausdruck gesellschaftlich wie historisch bedingter, psychosozialer Mentalitäten ist, mimetisch präziser dazustellen, als dies durch die orthografische Norm möglich wäre. Die damit erreichte Nähe zur Wirklichkeit bedeutet anders herum für den Leser und sein Verstehen die Überwindung eines Widerstands, die den Umstellungen und Umformungen durch ein Schreiben, so wie man spricht, gegenüber dem normierten Schriftbild geschuldet sind.

Jirgl arbeitet sich als Autor folglich an neuen Möglichkeiten der narrativen Signifikation ab, die sich, wie im Zusammenhang des emergenten Hervorbringens durch Akte der Versprachlichung (Koschorke) gezeigt, im Sinne einer Rückkopplung zwischen der Sphäre des Bezeichneten und dem bezeichnenden Subjekt (empirischer Autor) beschreiben lässt. Angesprochen wird damit auf die Fähigkeit des literarischen Textes »in mannigfaltigen Bereichen der Wirklichkeit sich ansiedeln und somit immer neue Signifikanten entdecken zu lassen [...]. «" Dabei verknüpft der alphanumerische Kode zwei Weisen der Darstellung von Wirklichkeit, die lineare der Buchstabenwörter und die »insulär[e] $\aleph^{10}$ der Ziffern. Die Fusion der beiden Wirklichkeiten bzw. ihrer Darstellungsweisen findet also primär, anders als bisher gedacht, nicht auf der Ebene der im literarischen Text erzählten Welt(en) statt, sondern setzt darunter auf der Ebene des Kodes an, in dem diese Welt(en) transportiert werden. ${ }^{11}$

\footnotetext{
$8 \quad$ Ebd., S. 69.

9 Ebd., S. 62.

10 Ebd., S. 55.

11 Jirgl begründet dies mit folgendem Argument aus der Neurophysiologie: »Man hat in der Hirnforschung erkannt, daß beispielsweise das Lesen eines von links nach rechts zu einem Satzende hin geradlinig verlaufenden, alphabetischen Textes andere neurophysiologische Vorgänge auslöst, als das Lesen (Entziffern - Erkennen) einer beliebigen mathematischen Formel. Bereits die einzelne Ziffer inmitten eines alphabeti-
} 
Jirgl trägt damit einer Tendenz Rechnung, die sich etwa seit einem halben Jahrhundert in der medientechnischen Entwicklung einer »Rückkehr zum Bild (von den Piktogrammen bis zu den Computeranimationen der virtual reality)« und dem gleichzeitigen »Vorausschritt zu den Zahlen (denn Computer waren und sind bloße Rechenmaschinen) ${ }^{12}$ manifestiert. Die Technik der numerischen Computation ermöglicht dabei sogar die Reversibilität beider Darstellungsformen ineinander. Ein Bild oder auch Musik kann in binär kodierte Ziffernkolonnen rückübersetzt werden und umgekehrt. Dies sind die beiden Ebenen, Imagination und Kalkulation, die in der besonderen Typografie des Schriftbilds ihren Ausdruck finden. ${ }^{13}$ Dadurch, dass der Text einen anderen Zeichenkörper erhält, wird zum einen die in den Buchstaben und Ziffern ursprünglich "geborgene Physis «, ${ }^{14}$ ihr bildhafter Charakter, erneut evoziert, zum anderen die zeitliche Dimension anders artikuliert als durch syntaktische Relationen, temporale Konjunktionen und verbale Beugungsformen, nämlich etwa durch »u« anstelle von »und« bei Gleichzeitigkeit oder der Verwendung von Ziffern, etwa der »1«, allgemein bei Betonung der Eile bzw. der zeitlich knappen Bemessung von Vorgängen. ${ }^{15}$

Textbedeutung wird somit nicht nur auf der Ebene der inhaltlichen Darstellung transportiert, sondern durch die Einführung neuer Zeichenebenen in den Text. Wie Jirgl erläutert, besteht die Motivation für eine solche innovative Umformung des typografischen Erscheinungsbildes der Schrift in dem, was er den »Pathos des Körpers ${ }^{16}$ nennt. Dessen ganzheitliche Vorstellung werde im Prozess des Schreibens zerstört und durch eine libidinöse Besetzung des Schriftkörpers, »die mimetisch veränderte Schriftformen hervorbringt«, die Rede ist auch von »Mutationen «, auf den als »erotischen Körper ${ }^{17}$ begriffenen Text übertragen. Wenngleich mit dem Körper-Term, auch dem des erotischen Körpers, in erster Linie die Entität des Textes mit den darin evozierten fiktionalen Figuren und Wirklichkeiten gemeint ist, wird das damit verbundene

schen Testes zwingt den Leser in seiner Lesetätigkeit zum »Umschalten« von der linearen Wirklichkeit der Buchstabenwörter zur »insulären «der Zahlen. Mit einem Satz gesagt: Der alphanumerische Code widerspiegelt die beiden Wirklichkeitsbedürfnisse des auditiv und visuell in der Welt seienden Menschen.«Ebd., S. $57 \mathrm{f}$.

12 Ebd., S. 63.

13 Vgl. ebd., S. $63 \mathrm{f}$.

14 Ebd., S. 62.

15 Vgl. ebd., S. 70.

16 Ebd., S. 65.

17 Ebd. 
Pathos ganz gewiss über eigene körperliche Erfahrungen und Erlebnisse des empirischen Autors vermittelt sein, denn ohne einen solchen, über den Körper vermittelten Kontakt zur Wirklichkeit wäre es gar nicht denkbar, mit der verwendeten Semantik sinnvoll zu operieren. Erotik kann man sich sozusagen nicht nur vorstellen, man muss sie zuvor auch erlebt haben.

Es muss aus diesem Grund die Kategorie der Nullstelle des Diskurses um einen Aspekt erweitert werden, der mit eben jener Motivation zur Schaffung der spezifischen Typografie und der dazugehörenden Semantik zusammenhängt. Denn anders als in den bisher besprochenen Texten ist diese weder durch eine Mise-en-abyme-Struktur, noch durch die Identität eines Eigennamens als solche im Text erkennbar. Zwar finden sich, ähnlich wie in Morshäusers Berliner Simulation, zahlreiche Bezüge zur Biografie des empirischen Autors, sofern die in seinen Texten etwa Abschied von den Feinden oder Hundsnächte verhandelten Schicksale mit den dazugehörenden politisch-gesellschaftlichen Hintergründen und landschaftlich-topografischen Gegebenheiten aus eigenem Erleben stammende Abbilder der DDR-Vergangenheit darstellen. ${ }^{18}$ So ließe sich auch mit ANH konstatieren, dass das objektive Geschehen im Leben des Autors zu einem miterzählten Gegenstand seiner literarischen Texte wird, doch bliebe dabei unberücksichtigt, dass die sonst kryptisch wirkenden

18 Den authentischen Bezug der beiden genannten Texte stellt Axel Kahrs, der ehemalige wissenschaftliche und künstlerische Leiter des Künstlerhofs Schreyahn, in seinem Nachwort zu Gewitterlicht heraus: „1953 in Ostberlin geboren, kam Jirgl noch im gleichen Jahr zur Großmutter nach Salzwedel. In dieser altmärkischen Stadt, weniger als zehn Kilometer Luftlinie von Schreyahn entfernt und doch durch die Grenze unerreichbar, wuchs er bis zum Umzug nach Ostberlin 1964 auf. In Salzwedel weckte eine Lehrerin sein Interesse für Sprache und Literatur, aufmerksame Leser finden in Jirgls Texten immer wieder Bruchstücke der Erinnerung aus der Stadt an der Jeetze. Die Bombardierung eines Zuges mit KZ- Häftlingen am Kriegsende, für Salzwedel historisch belegt, durchzieht als >Großer Dunkler Zug`den Roman >Hundsnächte` (1997), und auch ein weiteres Motiv stammt aus der Region: Ein sterbender Mann in einem Haus, das vom Abrissbagger bedroht wird. Jirgl verarbeitet hier die im DDR-Sperrgebiet gängige Praxis der Grenztruppen, zu nahe am Zaun gelegene Dörfer vollständig abzureißen, die Bewohner wurden zwangsumgesiedelt. Wer im Grenzgebiet zwischen der Altmark und dem Wendland unterwegs ist, findet auf den Grundstücksflächen der ehemaligen Dörfer Groß-Grabenstedt, Stresow oder Jahrsau (das Jirgl vor Augen stand), Spuren dieser >Grenzsicherung`, Gedenksteine und Mahnmale versuchen die Erinnerung an eine politische Cewaltmaßnahme wachzuhalten, die Jirgl schon in seinem Roman >Abschied von den Feinden< (1995) thematisiert hatte.« Ebd., S. 80 f. 
Formen des typografischen Schriftbilds, wie erläutert, mehr mit den Umständen ihrer Entstehung zu tun haben, als man denken würde. So ist nicht nur das libidinöse Begehren des Autors, wie er selbst erläutert, darüber vermittelt und in einem energetischen Sinn, »im Wiedererscheinen des Körpers (der Lust) als Text «, ${ }^{19}$ im Sinn eines Antriebs für das Schreiben in diesen übertragen, und damit eine Bedingung sine qua non seiner Entstehung. Hinzu kommt ein moralischer Impuls, der sich mit dieser "größtmöglichen Subjektivität des Textes, sowohl dem Inhalt als auch der Form (Erlebnis- und Ereignissubjektivität) gemäß ${ }^{20}{ }^{20}$ verbindet.

Es geht dabei, wie in folgendem Zitat deutlich wird, um den Zusammenhang von authentischer, lebensnaher Darstellung und der Evokation gesellschaftlichen Unrechts, von dem angenommen werden kann, dass die besondere Eigenart von Jirgels Texten sich darin wesentlich begründet:

»Durch Zeichenebenen im Text schaffe ich die Nähe und die Intensität zu meinen Figuren und ihren Wirklichkeiten, die ich, so genau wie möglich, in der Verschriftlichung suche. Nähe und Genauigkeit sind, wie man weiß, Synonyme. Eindeutigkeit der Lebenskonturen, so weit zugespitzte Situationen, um Entscheidungen zu ermöglichen - das sind wichtige Kriterien für meine Arbeit. [...] Ich suche mit meiner Sprache und meiner Text-Machart nach einer Ausdrucksmöglichkeit, das in den sozialen und mentalen Wirklichkeiten bestehende Unrecht zu benennen, zuzuspitzen, um es zu verneinen! « ${ }^{21}$

Musterhaft findet sich diese Intention in dem Nachwende-Roman Abschied von den Feinden am Beispiel eines Bruderpaares realisiert, dessen Lebensgeschichten aus je wechselnder Perspektivik bis in die Zeit nach dem Mauerfall erzählt werden. Es kommt dabei all das zur Sprache, was durch den Anschluss der untergegangenen DDR an die gesellschaftliche Ordnung der Bundesrepublik an geschichtlichen Altlasten und ungelebten persönlichen Utopien in den Köpfen der Menschen durch die Konfrontation mit einer in kurzer Zeit völlig veränderten politischen und ökonomischen Wirklichkeit plötzlich zutage tritt. Dies reicht von der erotischen Konkurrenz der um die gleiche Frau rivalisierenden Brüder, die, wie alle Figuren des Textes namenlos bleibt, und als »Frau mit dem Gesicht einer weißen Füchsin ${ }^{22}$ beschrieben wird, bis hin

\footnotetext{
19 Ebd., S. 62.

20 Ebd., S. 66.

21 Ebd., S. 65 f.

22 Reinhard Jirgl: Abschied von den Feinden, München: dtv 1998, S. 32 und passim.
} 
zur gesellschaftlichen Benachteiligung unangepasster, ihre individuellen Ziele verfolgender Menschen durch den allgegenwärtigen Staat. Es setzt sich fort mit der Vertreibungsgeschichte von Flüchtlingen aus den ehemaligen deutschen Ostgebieten und dem Verfall der durch Umsiedlung entvölkerten Ortschaften im Sperrgebiet der DDR-Grenze zur BRD.

All das wird unter Verzicht auf eine zentrale Instanz aus wechselnden Perspektiven (des jüngeren und des älteren Bruders sowie des kollektiven Wir einer Kleinstadt in Mecklenburg, in dem die Adoptiveltern des Brüderpaars, Flüchtlinge aus dem Sudentenland, gelebt haben) berichtet. Es entsteht auf diese Weise ein Kontinuum, das aus fraktal ineinander geschobenen Schauplätzen mit den dazugehörenden Erzählperspektiven besteht, sich in Analogie zur Chaostheorie gewissermaßen an den Instabilitätspunkten verzweigt und mit einer übergangslosen Transition in einen anderen Phasenraum des Erzählens eintritt. Erzeugt wird so ein Bewusstseinsraum, der vor allem zum Ende des Textes in den Projektionen der Figur des älteren Bruders - dieser war noch vor dem Mauerfall in den Westen ausgereist und befindet sich nun auf der Suche nach seiner Vergangenheit in Person »der Frau mit dem spitzen Füchsinnengesicht ${ }^{23}$ in einem auf freier Strecke stehendem Zug - mit schneller werdendem Rhythmus Orte und Zeiten des Erinnerns wechselt. Indem das physikalische Raum-Zeit-Kontinuum erzählerisch >gelöchert‘ wird, stellt die Literatur - dieses Mal wieder auf der Ebene der erzählten Welt(en) erneut ihre Fähigkeit zur Schaffung bisher nicht gesehener, welterzeugender Zusammenhänge unter Beweis. Der Leser erfährt auf diese Weise nicht nur vom Schicksal der Frau, ihrer Ehe mit dem reichen Chefarzt einer Berliner Klinik, ${ }^{24}$ der zugleich Stasiärzte ausbildet, ihrem Studium der Geschichte von ihm protegiert, der traumatischen Flucht von der Beerdigung ihres Vaters, ${ }^{25}$

23 Ebd., S. 15 und passim.

24 Vgl. ebd., S. 184.

25 Vgl. S. 60 f. und 239. Dies ist zugleich ein Beispiel für eine Mehrfacherzählung, bei der eine Episode, die dadurch eine besondere Bedeutung bekommt, wiederholt erzählt wird. Gleiches gilt auch für die Szenen der Misshandlung der Mutter, den Sturz des jüngeren Bruders von der Klippe, die Rückkehr des älteren Bruders in die Kleinstadt u.a. mehr. Vgl. zum Begriff der Mehrfacherzählung Sabine Sistig: Wandel der Ich-Identität in der Postmoderne. Zeit und Erzählen in Wolfgang Hilbigs »Ich« und Peter Kurzecks »Keiner stirbt«, Würzburg: Königshausen \& Neumann 2003, S. 49. Mehrfacherzählungen sind nicht nur typisch für Reinhard Jirgels Schreiben, sondern auch charakteristisch für das Werk von Wolfgang Hilbig, Peter Kurzeck und Thomas Melle. Es kommt darin das Zeiterleben des erzählenden Subjekts zum Ausdruck, das eine 
der späteren Einweisung in die Psychiatrie, ${ }^{26}$ sondern auch von der merkwürdigen Koinzidenz, die sie durch Vermittlung ihres Ehemannes in die gleiche Kleinstadt führt, in der auch die Adoptiveltern der beiden Brüder gelebt hatten. ${ }^{27}$ Dies kann als Beispiel dafür verstanden werden, wie die herkömmliche erzählerische Kontinuität fraktal gebrochen, die Kohärenz in der Folge an vielen Stellen durch Mehrfacherzählungen und seltsame Verdoppelungen von Identität zunächst unterhöhlt, damit in Frage gestellt, ${ }^{28}$ aber dann wiederum bestätigt wird, indem gerade dieser Effekt als Ausdruck einer erlebten Wirklichkeit fungiert, die sich ihrerseits eben nicht, wie vom Staat propagiert, als einheitlich, sondern als multipel und vielgestaltig erweist.

Der Wunsch nach der Offenheit von Möglichkeiten anstelle des einen zu erreichenden Ziels, das stellvertretend auch für die Geschichtsutopie des marxistisch-leninistischen Denkens steht, wird dem älteren Bruder vom jüngeren an einer Textstelle anlässlich einer erinnerten Reise sogar explizit zugeschrieben. Dort heißt es:

»Über lange Jahre hinweg weiß ich einen Wunsch in ihm: Während einer Reise aus dem Zug, dessen Fahrt auf der Strecke ins Stocken geriet, einfach auszusteigen - an beliebigen Orten, irgendwo dort, wo das Halten grad geschah. Das 1, festgelegte \& geplante Ziel wäre aufgegeben zugunsten der Möglichkeiten von vielerlei Wegen. ${ }^{29}$

Schon darin scheint, hier übertragen auf ein mögliches Handeln, das Programm systemischer Komplexität auf, wie es etwa in der Chaosforschung entwickelt wurde und sich, wie gezeigt, auch in der sprachlichen Gestaltung

Identitätsdiffusion durchmacht und die Zeit nicht linear-chronologisch (historisches Bewusstsein), sondern zyklisch (erlebte Erinnerungsstruktur) erlebt. Dadurch, dass die Episoden in variierenden Kontexten wiedererzählt werden, lässt sich, wie in Kap. VIII erläutert, auch von einer nachträglichen Bearbeitung eines bereits Erlebten sprechen, das jeweils neu getriggert bzw. rückgekoppelt wird. Vgl. R. Jirgl: Abschied von den Feinden, S. $250 \mathrm{f}$.

27 Vgl. ebd., S. $264 \mathrm{f}$.

28 Dies betrifft etwa einen Doppelgänger des jüngeren Bruders, der in der Küstenstadt im Norden der Republik, nachdem er vergeblich versucht hat, die Beziehung zu der Frau mit dem Gesicht einer Füchsin zu erneuern, einen ähnlichen Unfall erleidet wie dieser: „Übrigens lag er damals nicht weit von der Stelle an der Sie von der Steilküste-oben abgestürzt sind \& Wir sie gefunden haben ?!Ist solch 1 Zufall nicht wirklich komisch....«. Ebd., S. 270. 
des Romantextes niederschlägt. Man darf annehmen, dass dieser theoretische Hintergrund dem empirischen Autor, der von Beruf studierter Elektroingenieur ist, zur Zeit der Niederschrift des Romans bekannt gewesen sein dürfte. So verwendet er auch den Begriff der "Fraktale[...] $\aleph^{30}$ bei der Beschreibung einer Wagenspur im Sand, auf die der ältere Bruder nach dem Ausstieg aus dem stillstehenden Zug im ehemaligen Grenzgebiet trifft.

Doch ist es nicht nur dieses Detail, das als Synekdoche für die Anlage des gesamten Romans stehen mag, durch den sich in vielfältiger Weise die Spur der Erinnerung zieht, die nicht zur Ruhe kommen will, das Vergangene nicht gewesen sein lässt, sondern in schockartigen Bildern immer von neuem evoziert. Dieser Prozess beginnt mit einer frühen Kindheitserinnerung, in der die Mutter im Beisein der beiden Brüder von Stasischergen in der Berliner Wohnung der Familie misshandelt und in der Psychiatrie interniert wird, nachdem ihr Mann zum Klassenfeind in den Westen übergelaufen ist. ${ }^{31}$ Jene Episode, die sich mit anderen Erinnerungen an die Wohnung der Adoptiveltern überlagert, und vom jüngeren Bruder als Erzähler dem älteren als dessen eigene Erinnerung zugeschrieben wird, fungiert dabei als Urszene für das, was später von der Stimme des toten Vaters als »Explosion der Erinnerung « ${ }^{32}$ bezeichnet wird. Die damit gemeinte, ungezügelte Proliferation vergangenen Erlebens, die das Bewusstsein überschwemmt und sich dabei assoziativ mit den Komponenten der erzählten Gegenwart verknüpft, führt dazu, dass sich die Erzählsprache monologisierend verselbstständigt und dabei als anund abschwellender Strom von Bildern und Szenen (Satzkaskaden) den Leser, mitunter über ganze Seiten hinweg, bis an die Grenzen seines tropologischen und konkatenativen Vorstellungsvermögens treibt. Die Kaskaden von Möglichkeiten, die durch die Migration der Nullstelle in den Diskurs ausgelöst werden, erscheinen hier nicht als Potenz, die, wie im Fall der alternativen Chronologien, erst in der Interaktion zwischen Text und Leser manifest wird, sondern in den bizarren Wort- und Satzkaskaden, d.h. in der Materialität ihrer textuellen Entfaltung selbst. Dabei erweist sich auf Ebene der Diegese der jüngere Bruder als Quelle der Erzählung, sodass selbst die Episoden, in denen der ältere Bruder spricht und handelt, in der Fantasie des jüngeren ihren

30 Ebd.

31 Vgl. ebd., S. 47-52.

32 Ebd., S. 99. 
Ort haben und von dort diesem zugeschrieben werden. ${ }^{33}$ Damit wird offensichtlich, dass es sich bei dem erzählten Geschehen nicht um Wirklichkeit im Sinn gegebener Realität handelt, die dann nur mimetisch abzubilden wäre. Vielmehr geht es, wie hier deutlich wird, um die Darstellung innerer Wahrnehmungen - Träumen, Fantasien und Erinnerungen -, von denen man sich zuvor noch fragen konnte, wie es dem Autor gelingt, dieser inneren Welt den gleichen oder gar einen höheren Stellenwert einzuräumen als der Außenwelt, die sich als Umgebung objektiv zwischen die Menschen schiebt.

Erzähltheoretisch handelt es sich dabei um eine weitere Komplexitätsform der Metalepse, die Unlogische Heterarchie (verwickelte Hierarchie), bei der, wie Sonja Klimek schreibt, »eine Figur auf einer bestimmten narrativen Ebene [...] durch einen Darstellungsprozess (z.B. durch das Schreiben eines Romans) Macht über Figuren ihrer eigenen diegetischen Ebene aus[übt]. $\aleph^{34}$ In Jirgels Abschied von den Feinden ist es der jüngere Bruder, der durch die Darstellung seiner inneren Wahrnehmungen gewissermaßen Kontrolle über eine andere, präexistente Figur seiner eigenen diegetischen Ebene, in diesem Fall seinen älteren Bruder, erlangt. Dadurch, dass der jüngere Bruder sich in seiner Rolle als Urheber des Erzählvorgangs zu erkennen gibt, kommt es zwar, ähnlich wie bei der Möbiusband-Erzählung, der anderen komplexen Form der Metalepse, zu einer illusionsbrechenden Wirkung, die allerdings durch den fraktalen Perspektivwechsel des Erzählschauplatzes und der grammatischen Person, bei dem auch der ältere Bruder in der Ich-Form zu Wort kommt, nicht dauernd aufrechterhalten wird und deswegen zeitweise aus dem Bewusstsein des Rezipienten verschwindet. Diese Konstruktion ist deswegen paradoxal, weil jene Differenz, die durch die Markierung des Erzählakts formal eingeführt wird, im Erzählprozess selbst zu einer Durchdringung der Erzählebenen führt, die eine klare Identifikation der je mit dem Erzählen verbundenen Identität sehr erschwert. So wird der Unfall des jüngeren Bruders - der Sturz

33 Dies zeigt sich im 4. Kapitel des Romans, wo der jüngere Bruder erklärt: »1. Maßnahme. Halten auf freier Strecke. Erst langsam registrierte das ans Tempo des Zuges gewöhnte Cehirn diese Veränderung, den Stillstand des Zuges. In den vom Dröhnen des Fahrtwindes hypnotisierten Kopf kehrte plötzlich Stille ein. [...] Lasse ich die Schritte seiner Gedanken beginnen, die Leere des Augenblicks betreten.« Ebd., S. 31. Die Rückkehr des älteren Bruders, das Halten des Zuges auf freier Strecke mit allem, was sich daran anschließt, bis hin zu seinen Gedanken, erweist sich somit als Fantasieproduktion des jüngeren.

34 S. Klimek: Paradoxes Erzählen, S. 70. Gemäß der Typologie bei Klimek ist dies eine Metalepse des Typs 3b. Vgl. ebd. sowie ebd., S. 382-385. 
von einer Klippe - und sein Verbleib im Krankenhaus besagter Kleinstadt (als Ort seines Sprechens) zunächst einführend von einem anonym bleibenden Ich-Erzähler berichtet, ${ }^{35}$ was der These vom jüngeren Bruder als Urheber der innerdiegetischen Erzählung(en) zu widersprechen scheint. Dies geschieht jedoch mit den gleichen Mitteln des metaleptischen Hervorbringens und der Distanzierung einer von der erzählenden Instanz verschiedenen Figur, mit denen im weiteren Verlauf der jüngere Bruder die Figur des älteren aus sich erzeugt (»Ich lasse ihn [...]«), ${ }^{36}$ sodass man von einer Verdoppelung, tendenziell gar Vervielfältigung dieser Aussagestruktur bzw. einer Reversibilität der damit verbundenen (Sprecher-)Perspektivik ausgehen kann, ${ }^{37}$ wie sie auch in den miteinander rückgekoppelten Beobachtungskaskaden des Romans Buenos Aires. Anderswelt vorliegt. Später erweist sich dann eben auch genau dies, als der jüngere Bruder im Krankenhaus fantasierend umgekehrt den älteren als seinen Erzeuger konstruiert. ${ }^{38}$

Nun lässt sich diese Konstellation des Erzählens mit einem Paradoxon aus der Hirnforschung in Zusammenhang bringen, dessen Entfaltung die daraus entstehende Verwirrung erklären kann. Es handelt sich dabei um das Paradox der verschwundenen Welt (1) sowie einer zweiten damit zusammenhängenden Antinomie, die als sogenanntes Homunkulus-Problem (2) in der Neurobiologie bekannt ist: ${ }^{39}$ Wenn all das, was ich denke, wahrnehme, mir vorstelle, wie die Neurobiologen glauben, vom Gehirn erzeugt wird, wo ist dann der epistemische Ort dieser inneren Wirklichkeit bzw. der mit diesen Wahrnehmungen und Vorstellungen korrespondierenden Außenwelt? Wenn

35 Vgl. R. Jirgl: Abschied von den Feinden, S. 15 f.

36 Ebd. und passim.

37 Vgl. dazu meine Ausführungen zur Konvertibilität der Sprecherperspektive (veritative Symmetrie) in den Erzählungen von Wolfgang Hilbig, in: A. Steiner: Das narrative Selbst, S. 52-54.

38 Dies wird an folgender Stelle deutlich, wo der jüngere Bruder das eigene Sprechen als Falle empfindet: »]etzt beginne ich zu sprechen. Beginne meine Falle zu stellen für seinen Irrtum. Er hat mich aus seinen Wörtern entworfen. Mich in die Wörter geworfen. Hinabgestürzt diese 1 Klippe. Bis auf den Grund. Mich hineingestellt in die grenzenlosen Cefilde des Raunens + die Maßlosigkeit der Verlockungen seiner Wörter. Die er als die seinen nicht wiedererkennt. Zu einem Double von sich selbst. Blind. Stumm. Unfähig sich zu bewegen. Allein. Zum Hören verdammt. Er wollte durch mich die Frau erfahren. Meine Wörter, aus seinem Sprechen, sollten Das Geheimnis sprechen. Für ihn. Das sie, die Wörter erfahren mußten. «R. Jirgl: Abschied von den Feinden, S. 227.

39 Vgl. G. Roth: Das Gehirn und seine Wirklichkeit, S. 21 f. sowie meinen Aufsatz »Eine andere Gegenwart?«, in: Revista de Filologia Alemana 22(2014), S. 14-16. 
es zwei Welten gibt, eine Außenwelt der Gegenstände und eine zweite Welt der Wahrnehmung in meinem Gehirn, so entspricht dies jedoch überhaupt nicht meinem Erleben. Die Gegenstände sind sozusagen nicht in meinem Gehirn, sondern ich sehe sie draußen, um mich herum wie den Großteil meines Körpers. Entweder ist also die Annahme falsch, dass unsere Wahrnehmung im Gehirn entsteht oder es gibt da draußen gar keine Welt der Gegenstände, die von uns wahrgenommen und erlebt wird (1). Nimmt man nun an, dass unsere Erlebniswirklichkeit tatsächlich im Gehirn entsteht, so müsste aus neurobiologischer Sicht die Wahrnehmung der räumlichen Umgebung mit den Gegenständen und meinem Körper darin sich zugleich als Szene in meinem Gehirn abspielen, das sich in meinem Kopf befindet, der wiederum als Teil meines Körpers in diesem Raum anwesend ist, was wiederum, diesmal als verdoppeltes Szenario, von meinem Gehirn erzeugt wird. Wie kann aber das Gehirn, das unsere Wirklichkeit hervorbringt, zugleich ein Teil der Welt sein, aus der diese Wirklichkeit besteht (2)?

Wie man sieht, berührt sich die Vervielfältigung des Wahrnehmungsszenarios, die nie aus dem geschlossenen, selbstreferenziellen Zirkel der Homunkulus-Struktur entkommt und folglich nie zu einer wahrnehmungsunabhängig bestehenden, sozusagen objektiven Wirklichkeit aufschließt, mit dem Erleben des Erzählers im Roman Herr Gustafsson persönlich. Wie ein mittelalterlicher Homunkulus findet auch dieser keinen Zugang zur Wirklichkeit, bis er den Moment des Wahrgenommenwerdens durch die Philosophie-Dozentin Johanna Becker erlebt. Der glückliche Zufall dieser Begegnung eröffnet einen Weg, um der infiniten Vervielfältigung des Beobachterszenarios, wie sie im Homunkulus-Problem auftritt, zu entgehen nämlich durch rückgekoppelte Interaktion im Sinne allgemeiner Rekursion. In Abschied von den Feinden wird nun die epistemische Differenz zwischen selbst- und fremdreferenziellem Bezug des Erzählens durch entsprechende Einschübe des jüngeren Bruders (»Ich lasse ihn ...«) zwar markiert, doch kommt es, anders als in Gustafssons Roman, nicht zu einer kommunikativen Zuwendung, die den Zustand des Abgetrenntseins tendenziell aufhebt und es dem Erzähler ermöglicht, in eine mit anderen geteilte Welt einzutreten. Stattdessen steht das Bewusstsein von Tod und Zerfall, für das auch das wiederkehrende Motiv der Fliegenschwärme steht, ${ }^{40}$ bereits am Anfang der Erzählung und zieht sich als bestimmendes Sujet durch den gesamten Text. 
Dies betrifft sowohl den Tod der Frau mit dem Gesicht einer weißen Füchsin, die unter ungeklärten Umständen ums Leben kommt, ${ }^{41}$ als auch den Verlust der Adoptiveltern und der Mutter, der jeweils schon länger zurückliegt. Es ist daher nur folgerichtig, wenn der jüngere Bruder als Erzähler des älteren diesen bei seiner Rückkehr in die Kleinstadt im Norden nie ans Ziel gelangen, sondern ihn aus dem stehenden Zug vorzeitig aussteigen lässt, ${ }^{42}$ denn das Motiv seiner Reise, ein Wiedersehen mit der bereits toten Frau, ist damit ja hinfällig. Auf seinem weiteren Weg zu Fuß stößt er dann auf ein zurückgelassenes Dorf, in dem er die zwangsgeräumte und dem Verfall preisgegebene Ortschaft wiedererkennt, wo einst die Adoptiveltern nach ihrer Flucht aus Schlesien bei einem Bauern untergekommen waren, wenngleich es nicht der identische Ort ist. Die damals beschlossenen staatlichen Maßnahmen im Grenzgebiet wirkten sich in ihrer Konsequenz eben flächendeckend aus und waren nicht auf einzelne Ortschaften beschränkt. Damit wird zudem eine textuelle Ebene angesprochen, die in Form von Auszügen aus einem Buch über die spanische Conquista im heutigen Mexiko der Erzählung einen weiteren Schauplatz hinzufügt. In diesem Buch, das die beiden Brüder in ihrer Jugendzeit viel gelesen hatten und in dem der ältere Bruder während der Zugfahrt nun wieder liest, wird geschildert, wie einer der Anführer der Konquistadoren auf dem Feldzug gegen die Azteken von Indios auf grausame Weise zu Tode gemartert wird. ${ }^{43}$ Dabei erscheinen der politische Anschluss und die Übernahme der untergegangenen DDR-Wirtschaft durch westdeutsche Investoren als Replik auf die kolonialistische Landnahme der spanischen Konquistadoren. ${ }^{44}$

Es liegt schließlich nahe, den Tod der erotisch begehrten Person, der Frau mit dem spitzen Füchsinnengesicht, und die dadurch hervorgerufenen Erinnerungskaskaden und Fantasien, welche die genannten Komponenten miteinander verbinden, als Initial bzw. auslösendes Moment des Erzählens zu begreifen. ${ }^{45}$ Dabei wirkt sich die Modalität rückläufiger Kausalität in der Weise auf das Erzählen aus, dass ein bereits stattgefundenes Geschehen (Tod der Frau, Ende der DDR als staatliches Gebilde) nachträglich die Darstellung der

\footnotetext{
41 Vgl. ebd., S. 19 und passim.

42 Vgl. ebd., S. $236 \mathrm{f}$.

43 Vgl. ebd., Kap. 14, S. 206-223.

44 Vgl. ebd., Kap. 5, S. 35-40.

45 Vgl. »Bericht vom Sprechen«, ebd., S. 224-229, hier S. 225 unten.
} 
erzählerischen Gegenwart beeinflusst (Abbruch der Zugfahrt des älteren Bruders, Vorwegnahme des eigenen Todes durch diesen als Mörder, Schilderung der Nachwende-Realität vor dem Hintergrund der Geschichte des Konquistadors). Zugleich kommt durch die Vielzahl der beteiligten Komponenten die Mehrstelligkeit des Diskurses zum Ausdruck. Es zeigt sich damit wiederum, dass Erinnerung, Fantasie und gegenwärtige Wahrnehmung auf der Ebene der Diegese über die Produktion des Textes, verstanden als Sprechen des jüngeren Bruders, im Sinne der retroaktiven, unabschließbaren Besetzung des entzogenen Grundes der ursprünglichen Wirklichkeit (Iser) komplex miteinander rückgekoppelt sind. Das Paradox der verschwundenen Welt betrifft somit nicht nur die gegenwärtige Wahrnehmung, sondern vielmehr auch die mit dem Erinnern verbundenen Eindrücke, die ja selbst dann noch da sind, wenn sich die Welt so sehr verändert hat, dass den Eindrücken in der Wirklichkeit nichts bzw. kaum noch etwas entspricht.

All dies kann als Ausdruck von textueller Komplexität verstanden werden, die sich, wie gezeigt, bereits auf der Ebene der zeichenhaften Materialität des Erzähldiskurses manifestiert und, anders als bei den bisher behandelten Beispielen für komplexes Erzählen, nicht erst in der Interaktion zwischen Textinhalt und Leser entsteht. Es kommt allerdings ein Aspekt hinzu, der mit der selbstreferenziellen Verdoppelung bzw. iterativen Vervielfältigung des Szenarios zu tun hat, wie sie in der Homunkulus-Struktur zutage tritt, und neben der übergangslosen Transition zwischen den Schauplätzen dazu berechtigt, hier vom fraktalen Erzählen zu sprechen. Dies bedeutet umgekehrt für die Rezeption, dass es durch die aus dem Homunkulus-Problem resultierende, verwirrende Frage, wer im Erzählen wen hervorbringt, jeweils darauf ankommt, was das kognitive System des Lesers im Prozess der Textwahrnehmung ergänzt bzw. weglässt. Denn dieses arbeitet selektiv und ist weder für die Erfassung/Vergegenwärtigung einer Vielzahl möglicher Konstellationen noch eines hochgradig parallel verlaufenden Gesamtgeschehens ausgelegt. In einem etwas anderen Sinn, als zuvor im Zusammenhang der Unschärferelation dargestellt, ${ }^{46}$ erweist sich wieder, dass die Beobachtung/Wahrnehmung ihren Gegenstand, hier den literarischen Text, beeinflusst, was bei streng deterministischer Betrachtungsweise zu den Wicked problems führt, auf die im folgenden Abschnitt noch zurückzukommen sein wird. 


\section{Fazit}

Während die Beispiele komplexen Erzählens durch alternative Chronologien und die Rahmenstruktur der Erzählung bei Gustafsson bzw. Kermani sowie die Interaktion zwischen Jetztzeit und erlebter Vergangenheit bei Kluge das Subjekt als erzählendes gewissermaßen sogar konsolidieren und darüber hinaus zu neuen Möglichkeiten der Darstellung gelangen, fällt das Urteil im Fall des ersten und der beiden zuletzt besprochenen Texte weniger positiv aus, wohl weil das in ihnen entwickelte Szenario im Vergleich radikaler ist. Sowohl in ANHs Buenos Aires. Anderswelt als auch in Morshäusers Berliner Simulation und Jirgls Abschied von den Feinden findet das erzählende Subjekt nicht mehr zu einer kohärenten Wirklichkeitswahrnehmung und daher auch nicht zu einer kohärenten Identität zurück. Im Zuge der parodistischen Absage an die Möglichkeit einer irgendwie noch realistischen Darstellung von Wirklichkeit gleitet das Erzählen, besonders bei Herbst, in die Simulation hinüber, die, wie Baudrillard festhält, »sich niemals gegen das Reale austauschen läßt, sondern nur in sich selbst zirkuliert und zwar in einem ununterbrochenen Kreislauf ohne Referenz (référence) und Umfang (circonférence). « ${ }^{1}$

Wie Baudrillard weiter ausführt, wird im Zustandsraum der Simulation die traditionelle Form der Kausalität mit ihrer Unterscheidung zwischen Ursache und Wirkung, aktiv und passiv sowie Subjekt und Objekt obsolet, ${ }^{2}$ weil gewissermaßen alles gleichzeitig geschieht und schon da ist, ohne dass die Möglichkeit besteht, in den Prozess der Simulation einzugreifen oder anders, als von den Programmen vorgesehen, zu reagieren. ${ }^{3}$ Sobald die Differenz

1 Jean Baudrillard: Agonie des Realen, übers. aus d. Franz. von Lothar Kurzawa und Volker Schaefer, Berlin: Merve 1978, S. 14.

2 Vgl. ebd., S. 49.

3 Daraus ergibt sich die Frage, wie man dann überhaupt sprachlich, d.h. mit einer Subjekt-Objekt-Struktur sowie den entsprechenden Strukturen für Kausalität, Finalität und Bedingtheit, auf die Herausforderungen durch Komplexität reagieren soll, 
zwischen Realität und Illusion/Fiktion, zwischen Wahrheit und Schein/Täuschung/Lüge in der Spirale der Simulation verschwindet, ergibt sich auch für die Parodie das Problem der Referenz, ${ }^{4}$ das in der Berliner Simulation nur durch die (gespenstische, marionettenhafte) Bezugnahme auf das Referenzial der zeitgeschichtlichen Berliner Vergangenheit umgangen wird, die sich mit der Biografie des Ich-Erzählers resp. des empirischen Autors deckt. Wenn das, was parodistisch herabgesetzt oder konterkariert werden soll, letztlich selbst nur noch als Simulakrum existiert, läuft die Attacke ins Leere und wird selbstbezüglich wie das, worauf sie gezielt hatte. Dies resultiert daraus, dass das Imaginäre sich nicht länger mit dem Realen, sondern unmittelbar mit der Simulation austauscht. Wie gezeigt, geschieht genau das dem Ich-Erzähler Alban Herbst mit seinem digitalen Double Hans Deters, wie auch dem namenlos bleibenden Ich-Erzähler der Berliner Simulation mit der Berichterstattung in den Medien. Gleiches gilt für den jüngeren Bruder in Abschied von den Feinden, dessen Erzählen aufgrund der Homunkulus-Struktur selbstbezüglich bleibt. Obwohl es die Perspektiven zu vervielfältigen vermag, nimmt das sich entwickelnde Szenario den Charakter eines Simulakrums an, in dem der Erzähler im Sinne eines Phantasmas gefangen bleibt.

Hier zeigt sich, dass die Simulation in gewissem Sinn gefährlicher ist als das Reale, »denn sie läßt über ihr Objekt hinaus die Annahme zu, die Ordnung und das Gesetz könnten selber ebenso gut nur Simulation sein. $\aleph^{5}$ Durch diese erneute Drehung der Spirale droht die Grundlegung dessen, was als politische und gesellschaftliche Realität gilt, im Orkus der Simulation zu verschwinden. Dehnt man diesen Ansatz auf die Grundlagen von Logik, Syntax und Semantik aus, indem man sie mit Nietzsche nicht als Spiegel gegebener Wirklichkeit, sondern als kognitiv programmiertes Produkt auffasst, ${ }^{6}$ wird neben der politischen auch die referenzielle und epistemische Dimension der Simulation offenkundig. Dies wird dann zum unlösbaren Problem, wenn man die

die im gesellschaftlichen Leben heutzutage hauptsächlich in Form der Digitalisierung begegnen. Darauf hat auch Nassehi in der Wissenschaftstalkshow SCOBEL: »Ethik fürs Digitale«, 3sat, Sendung vom 03.09.2020, aufmerksam gemacht.

4 Vgl. J. Baudrillard: Agonie des Realen, S. 35.

5 Ebd., S. 35 f.

6 Mit diesem Aspekt hat sich Ralf Beuthan in seiner Studie zum Undarstellbaren in Film und Philosophie auseinandergesetzt. Ich habe darauf in einem Aufsatz über Annäherungen zwischen Erzähltheorie und Neurowissenschaft Bezug genommen, wo dieser Zusammenhang auch bereits hergestellt wird. Vgl. dazu A. Steiner: »Eine andere Gegenwart?«, in: Revista de Filologia Alemana 22(2014), S. 23. 
Simulation für alle Bereiche des Wirklichen verallgemeinert, eingeschlossen sich selbst. Angesichts der Geschlossenheit dieser Konstruktion, aus der es kein Entkommen gibt, solange man sich innerhalb der Logik von Simulation und Dissimulation bewegt, weil sie jede Äquivalenz, jeden Austausch mit dem Realen unmöglich macht, kommt es wieder darauf an, Kontextsensibilität $\mathrm{zu}$ entwickeln. Würde man umgekehrt der fortgesetzten »Torsion des Sinns ${ }^{7}$ dadurch zu begegnen versuchen, dass man das Möbiusband der Simulation noch einmal teilt, so entstünde »daraus eine zusätzliche Spirale, ohne daß die Reversibilität der Oberflächen [...] aufgelöst würde. $\aleph^{8}$ Wie sich am Beispiel von Gustafssons Roman gezeigt hat, mündet diese Strategie auf der Ebene der Rezeption jedoch auch in die Möglichkeit der operativen Erweiterung einer Geschichte hin zu alternativen Chronologien des erzählten Geschehens.

Man entkommt also nicht, indem man die Struktur als Repräsentation eines gegebenen Sinns einfach nur perpetuiert und dabei innerhalb des Closed circuit der simulierten Wirklichkeit(en) verbleibt. Vielmehr geht es darum zu begreifen, dass das Verständnis der Welt in der Interaktion, d.h. im Zwischenraum des Umgangs mit den Dingen jenseits der Simulation liegt, wie das Beispiel von Gustafsson gezeigt hat. Es käme also darauf an, dass eine Rückübersetzung aus der Komplexität digitaler Simulation bzw. dem Closed circuit der Homunkulus-Struktur in eine analoge Form stattfindet. ${ }^{9}$ Diese Rückübersetzung wird durch die (re-)produktive Aktivität des Subjekts gewährleistet, dem es dabei durch Lernen gelingt, das Paradigma der Repräsentation in Richtung Emergenz zu überschreiten. Intelligenz und damit intelligentes Verstehen und Verhalten wären dann zudem, wie Varela vorgeschlagen hat, »nicht mehr als die Fähigkeit des Problemlösens zu verstehen, sondern

7 J. Baudrillard: Agonie des Realen, S. 32.

8 Ebd.

9 Darauf verweist auch Nassehi in seinem Buch zur soziologischen Theorie der digitalen Cesellschaft allerdings in eher technischer Hinsicht: »]edenfalls ist Digitalität als eine Kombination von Vereinfachung und Komplexitätssteigerung anzusehen. Man bringt analoge Formen in eine digitale Gestalt, rekombiniert diese digitale Cestalt im Hinblick auf Strukturen und wendet diese dann wieder auf die analoge Welt an, aus der die Daten stammen, oder besser: von der her die Welt in Datenform verdoppelt wurde. [...] Jedenfalls setzt die Anwendung von Computern nicht einfach auf die interne Bearbeitung von Daten, sondern auf deren Rückübersetzung in analoge Formen.«Armin Nassehi: Muster. Theorie der digitalen Cesellschaft, München: C.H. Beck 2019, S. 34. 
als die Fähigkeit, in eine mit anderen geteilte Welt einzutreten. ${ }^{10}$ Von hier aus wird auch verständlich, warum die Idee transkultureller Kommunikation bei Welsch sich vom hermeneutischen Modell des Verstehens verabschiedet und sich dabei auf das konnektionistische Modell aus der Neurowissenschaft verlegt. Denn dies demonstriert ja auf der Basis der Informationsverarbeitung im Gehirn, wie auch außerhalb davon, etwa in der künstlichen Intelligenz, an sich unintelligente Bestandteile netzwerkartig »in angemessener Weise verknüpft, interessante übergreifende Eigenschaften zeigen «, ${ }^{11}$ wie Varela schreibt. Anstelle der Reduktion eines Problems auf seine Lösung wird der Möglichkeit des Eintritts in einen intersubjektiv geteilten Raum komplexer Pluralität der Vorzug gegeben.

Rückübersetzung in eine analoge Form bedeutet nun im Zusammenhang komplexen Erzählens, dass man komplexe literarische Formen/Muster, wie an den Beispieltexten erörtert, in eine sinnfällige Anweisung für ihre Lektüre zurückübersetzt. So kann der Begriff des Erlebnismodells, ${ }^{12}$ den Vilém Flusser in Analogie zu den Modellen der Simulation als Zweck literarischer Texte begreift, etwas anders, als vom Autor intendiert, d.h. nicht im Sinn der Ablösung der linearen Darstellungsform durch die digitale Simultaneität der technisch erzeugten Bilder, sondern vor dem Hintergrund einer Rückübersetzung der genannten Phänomene (Echtzeit, Simultaneität, Zeitumkehr) in den Kode der Literatur verstanden werden und so für diese erhalten bleiben. Die Literatur zeigt sich damit durchaus in der Lage, auf die Modelle der Simulation zu antworten, indem sie textuelle Muster entwickelt, die es dem Leser erlauben, die neuen, mit dem digitalen Kode verbundenen Phänomene im Prozess der Lektüre zu reproduzieren. Gelingt es umgekehrt, die alte, an Linearität orientierte Lesart und das daran gekoppelte Verständnis von Geschichte als sukzessiv-lineare Abfolge von Ereignissen in ein kybernetisches Bewusstsein zu transformieren, so wird der Leser dadurch in die Lage versetzt, nicht nur sein eigenes Netz aus Information und Bedeutung sondern auch »seine eigenen Zeitströme zu knüpfen. ${ }^{13}$ Auf diese Weise wäre einer einseitigen Umkodierung literarischer Inhalte in digitale Formate und der damit verbundenen Reduktion menschlicher Gefühls- und Erlebniswelten

\footnotetext{
10 F. Varela: Kognitionswissenschaft - Kognitionstechnik, S. 111.

11 Ebd., S. 58.

12 Vgl. V. Flusser: Die Schrift, S. 66-70.

13 Ebd., S. 136.
} 
vorgebeugt. Vielmehr kommt es so zu einer echten, wechselseitigen Interaktion zwischen Literatur und digitaler Simulation, bei der die Komplexität der in den neuen Medien repräsentierten Wirklichkeit(en) durch Rückübersetzung in komplexe Muster literarischen Erzählens im Sinn von Flussers Erlebnismodell erst erschlossen werden kann. Wenn Flusser im Zusammenhang der Umkodierung/Übersetzung des linearen Kodes der Literatur in den netzwerkartigen der digitalen Simulation gar davon spricht, dass der Kode des Alphabets, mit ihm die lineare Schrift und das daran gekoppelte historische Bewusstsein, aus dem Gedächtnis der Menschheit gelöscht werden müssten, um den neuen digitalen Kodes Platz zu machen, ${ }^{14}$ so haben die Textanalysen gezeigt, dass auch der umgekehrte Weg möglich und sogar sinnvoll ist. Zwar kann dadurch die »Mehrfachkodierung von allem mit der Komplexität der Gleichzeitigkeit unterschiedlicher Hinsichten $\ll,{ }^{15}$ von der Nassehi spricht, nicht völlig desambiguiert werden. Doch durch die Rückübersetzung in den Kode der Literatur wird die Differenz zwischen verschiedenen, möglichen Bedeutungen als interne vom Subjekt im Prozess der Lektüre reproduziert. Sie kann auf diese Weise jedenfalls wahrnehmbar und dadurch womöglich auch besser handhabbar gemacht werden.

Während die Rückübersetzung in sinnförmige Information (für die Lektüre oder das Schreiben) bei Gustafsson in den alternativen Chronologien besteht, für Kermani in der Rahmenstruktur von Fiktion und Realität sowie dem Anspruch des Erzählens in Echtzeit, bei Kluge hingegen in der Interaktion von Jetztzeit und erlebter zeitgeschichtlicher Vergangenheit gekoppelt an die Idee alternativer Chronologien des geschichtlichen Verlaufs, wird die Suche nach einem weiteren, aus dem Schreiben/Lesen hervorgehenden Sinn (Emergenz) bei ANH und Bodo Morshäuser durch eine parodistische Absage suspendiert. Der Sprung zurück aus dem fraktalen Raum der Simulation in die analoge Welt will zumindest auf der Ebene des erzählten Geschehens nicht gelingen oder bleibt defizitär, sodass es nicht zu einer kohärenten Wirklichkeitswahrnehmung und der damit verbundenen Konsolidierung der Identität des erzählenden Subjekts kommen kann. Weder die erotische Strategie der Annäherung an Sally noch der Versuch der Verschmelzung der eigenen Identität mit der von Deters durch die kybernetische Operation des Paste-andCopy-Befehls bewirken für die Erzähler das, was durch sie erreicht werden soll. Gerade dadurch, dass Simulation und Realität ununterscheidbar werden 
und sich das Imaginäre nicht mehr mit der Wirklichkeit, sondern unmittelbar mit der Simulation tauscht, bleibt das Subjekt fragmentiert und findet sich nicht in einer mit anderen geteilten, neuen Wirklichkeit, die sich qualitativ von der überkommenen unterscheiden würde. Es wird vielmehr auf sich zurückgeworfen und kommt nicht in einer Weise zu sich selbst, die es erlauben würde, von einem damit erreichten, neuen Horizont emergenter Erfahrung zu sprechen. Ähnliches gilt für den doppelgesichtigen Erzähler in Abschied von den Feinden, der angesichts einer von Tod und Verfall geprägten Vergangenheit letztlich in den fraktalen Iterationen selbstbezüglichen Erzählens zu verschwinden droht. ${ }^{16}$

Doch was bedeutet dies für die mit dem komplexen Erzählen verbundene Möglichkeit des Lesers aus fixiertem Verstehen und den daran gebundenen Weisen von Erfahrung und Motivation aufzubrechen? Festzuhalten ist, dass auch in den anderen Erzähltexten die Vorstellung kohärenter Ich-Identität im Sinne der Einheit eines durch die Zeit hinweg Identischen als heuristische Fiktion entlarvt und dekonstruiert wird. Wenn Mieth darauf rekurriert, dass eine vereinfachte Sicht der Wirklichkeit durch komplexe Erzählwelten aufgeschlossen werden könne, ${ }^{17}$ so ist zu bedenken, dass es in keinem der besprochenen Erzähltexte darum geht, durch Nachahmung eines Vorbilds das eigene Verhalten (adaptiv) zu verändern. Wie sich in der Analyse herausgestellt hat, handelt es sich vielmehr darum, die in der Rezeption erkannten Modelle für eine neue Interpretation des eigenen Lebens zu nutzen. Dieser Prozess wiederum kann beim Leser durch die Wahrnehmung alternativer Kohärenzen und dem damit verbundenen Clash der Vorstellungen, welche die Lektüre komplexer Erzähltexte gewöhnlich begleiten, angestoßen werden. Wie im Fall der alternativen Chronologien kann dies im Sinn von Lommels Lebensvarianten geschehen oder, indem die Modelle den Leser anregen, das eigene Handeln in bestimmten Hinsichten praktisch zu verändern, sofern Anschlüsse an ursprünglich gar nicht intendierte Kontexte gesucht und gefunden werden.

16 So spricht der Erzähler am Ende des Textes von einer Reduktion des älteren Bruders auf eine punktförmige Existenz: »Er war kein Reisender mehr. Er war an einem Ort, der seinen Namen verloren hatte \& der niemals 1 Namen wieder bekommen würde. Der vielleicht zerfallen, in Dreck Stein \& Staub sich zurückverwandelnd, und schließlich verschwinden würde; ein Ort also, an dem er nicht gewesen wäre. Dort, in diesem langsam erbleichenden Ort, würde er, unvereinbar mit-sich u: seinen Bildern, 1 blinder Punkt sein, 1 Fleck. Der würde bleiben.«R. Jirgl: Abschied von den Feinden, S. 321.

17 Vgl. D. Mieth: Komplexes Erzählen, S. 105. 
In diesem Sinn können die behandelten Texte auch als transformative Erzählungen verstanden werden. Im gegebenen Zusammenhang haben sich so aus der Betrachtung des literarischen Textes als komplexem Phänomen Konnektionen zu verschiedenen Diskursen ergeben, aus der die Öffnung in Richtung Pluralität möglicher Deutungsanschlüsse mit produktiven Rückkopplungseffekten resultiert.

Durch die Erfahrung zunehmender Selbstwirksamkeit gelingt es möglicherweise, den mit Komplexität und deren Bewältigung verbundenen Wicked problems nicht nur innerhalb, sondern vor allem auch außerhalb der Literaturwissenschaft besser zu begegnen als bisher. Gemeint sind damit einmal die Übersetzungsprobleme zwischen den unterschiedlichen Logiken, Intelligenzen und Funktionen, von denen Nassehi mit Bezug auf die Ausdifferenzierung der gesellschaftlichen Verhältnisse und ihrer digitalen Strukturen spricht. Dem entspricht in der vorliegenden Studie die Kopplung verschiedener Diskurse mit je unterschiedlichen Erkenntnisinteressen und Methodiken auf dem Feld des komplexen Erzählens. Dann aber auch das bereits angesprochene Problem, wie man als sprachliches Wesen angemessen auf komplexe Verhältnisse antworten soll, die strukturell durch die Gleichzeitigkeit von Parallelprozessen gekennzeichnet sind. ${ }^{18}$ Dabei geht es sicher weniger

Vgl. FN 3 des laufenden Kapitels. Die disruptive Brechung zwischen programmgetriebener, virtueller und analoger Realität hängt vor allem damit zusammen, dass der Horizont des Wirklichen, der phänomenologisch besonders durch die visuelle und auditive Wahrnehmung erzeugt wird (Wahrnehmungshorizont), im fraktalen Raum der Simulation zerfällt und durch das ersetzt wird, was Virilio als »indirekte[...] Sichtbarkeit [...] des hindurch-sichtbaren Horizonts« bezeichnet. P. Virilio: Fluchtgeschwindigkeit, S. 41. Dieser kann zwar durch Datenhelm und -handschuhe auch dreidimensional und von hoher Auflösung sein, erreicht dabei jedoch nie die echte, direkte Sichtbarkeit des in die geografische Realität eingebetteten, analogen Wahrnehmungshorizonts visuell weitgehend euklidisch orientierter Subjekte. So kann es nicht unmittelbar zu einer Verschmelzung beider Realitäten kommen, denn das Subjekt interagiert zu gegebener Zeit entweder mit der einen oder der anderen »Welt«, wobei die Interaktion mit der einen durchaus Wirkungen in der anderen hervorruft, weil eben beide über das Subjekt, dessen Wahrnehmungen, Aktivitäten und Bedürfnisse miteinander vermittelt sind.

Dass es sprachlich mitunter so schwer fällt zu beschreiben, was an dieser Crenze zwischen analoger und digitaler Wirklichkeit mit einem geschieht, liegt eben daran, dass diese Grenze im bzw. durch das Subjekt hindurch verläuft. Ist das Bewusstsein an die virtuelle Simulation gekoppelt, können sich plötzlich die Verhältnisse zwischen Ursache und Wirkung, Subjekt und Objekt, aktiv und passiv umkehren. Typischerweise macht sich das dadurch bemerkbar, dass einem zumeist zwar nicht die Worte ganz, 
darum, alle komplexen Zusammenhänge, verstanden als Gleichzeitigkeit von parallel ablaufenden Prozessen, auch gleichzeitig denken zu wollen, um auf diese Weise als konvergentes Resultat den verloren gegangenen, umfassenden Überblick zurück zu gewinnen. Wie Friedrich Cramer gezeigt hat, geht dieser Überblick verloren, sobald es darum geht, die Interaktion des Menschen mit der belebten Umwelt zu beschreiben. Nicht nur durch die gleichzeitig parallel zu berücksichtigenden Prozesse und deren Wechselwirkungen, sondern vor allem durch den Umstand, dass das Subjekt selbst Teil des lebendigen Netzwerks ist, das wissenschaftlich erfasst werden soll. ${ }^{19}$ Die Interaktion bzw. ihre wissenschaftliche Darstellung bleibt aus diesen Gründen in wesentlichen Hinsichten unbestimmt, darum unvollständig und kann deswegen nie im Sinn eines Gesamtüberblicks objektiviert werden. Angesichts der Komplexität eines solchen, hochgradig parallelen Prozessgeschehens entpuppt sich

aber die Verben fehlen, die ja zwischen dem Subjekt und dem Objekt einer Aussage vermitteln und in dieser Struktur eine bestimmte Aktivität zum Ausdruck bringen. Kehren sich die Verhältnisse um und das System übernimmt an den Positionen, die zuvor in der Handlungsmacht des Subjekts gelegen waren, den Part des Agenten, kommt es zu entsprechender Verwirrung, die sich darin äußert, dass ein erlebtes Geschehen nicht mehr in herkömmlicher Weise der eigenen Aktivität oder den damit verbundenen Wirkungen zugeordnet werden kann. Der dadurch entstehende Autonomieverlust kann zumeist nicht ad hoc durch eigene Aktivität, sei diese sprachlich oder nicht, kompensiert werden.

Radikalisiert findet sich diese Verdoppelung der Welt in filmischen Szenarien wie Welt AM DRAHT (BRD 1973, R: Rainer Werner Fassbinder), MatriX (USA/AUS 1999, R: Lana und Lilly Wachowski,) und INCEPTION (USA/CB 2010, R: Christopher Nolan). Cezeigt wird darin jeweils, wie virtuelle und analoge Wirklichkeit, Real- und Traumwelt sich an den Schnittstellen per Datenhelm, Bioport oder Traumsharing im Bewusstsein des Subjekts, das daran angeschlossen ist, miteinander tauschen. In der literarischen Erzählung ist dieser Austausch schwerer zu signifizieren, da es im linearen Medium kaum möglich ist, den instantanen Wechsel der Bewusstseinszustände mimetisch, d.h. durch Metapher, Allegorie oder Montage, so zum Ausdruck zu bringen wie im Film. Komplex wird ein solches Szenario dann, wenn, wie in WELT AM DRAHT und INCEPTION, die einfache Verdoppelung sich fraktal fortsetzt. So könnte durch den Supercomputer Simulacron (WELT AM DRAHT) in der Simulation (Ebene 1) eine weitere Simulation (Ebene $n+1$ ) erzeugt werden, wie auch das Unbewusste durch einen Traum im Traum usf. (INCEPTION) Binnenzustandsformen hervorbringt, die wiederum miteinander interagieren, sodass es dabei zu einem Schichtenmodell, einer Mise en abyme oder einer netzwerkartigen Struktur kommt.

Vgl. Kap. XIII. 
schließlich auch die eingangs gestellte Frage, wie alles mit allem zusammenhängen mag, als rhetorische Falle, die sich aus den genannten Gründen gar nicht diskursiv beantworten lässt, weil sie sich der linearen Darstellung weitgehend entzieht.

Daraus lässt sich der Schluss ziehen, dass es vielmehr darum gehen müsste, sich mehr und mehr mit netzwerkartigem Denken vertraut zu machen. Dabei stünde Rekursion nicht nur für eine Rechenvorschrift innerhalb der Programmierung von Algorithmen, sondern vor allem auch für eine Bewegung des Denkens, sowohl in der Beschäftigung mit literarischen Texten, als auch in der Auseinandersetzung mit der Umwelt. Auf diese Weise kann es gelingen, die Interdependenzen zwischen den verschiedenen, simultanen Prozessverläufen besser verstehen zu lernen und Übersetzungsstrategien dafür in linear funktionierende Formate wie die Literatur zu finden. Diese Herausforderung betrifft sicher in gleichem Maße Literaturschaffende wie Literaturwissenschaftler. Daraus wiederum könnte sich angesichts der genannten Wicked problems, die sich eben sicher nicht durch den verloren gegangenen Überblick werden lösen lassen, weil Prozesskontrolle und -prognose streng genommen nur unter linearen Bedingungen gewährleistet werden können, ein neuer Modus operandi ergeben. 



\section{Quellenverzeichnis}

\section{Primärtexte}

Auster, Paul: 4321, London: Faber \& Faber 2017.

Goetz, Rainald: Abfall für alle. Roman eines Jahres [1999]. 3. Aufl., Frankfurt a.M.: Suhrkamp 2015.

Gustafsson, Lars: Herr Gustafsson persönlich [1971], übers. aus d. Schwedischen von Verena Reichel, Frankfurt a.M.: Fischer Taschenbuch 1985.

Herbst, Alban Nikolai: Die Verwirrung des Gemüts, München: List 1983.

Ders.: Buenos Aires. Anderswelt, Berlin: Berlin Verlag 2001.

Ders.: Kybernetischer Realismus. Heidelberger Vorlesungen, Heidelberg: Manutius 2008.

Hilbig, Wolfgang: »Der Heizer [1980]«, in: Ders., Erzählungen, Frankfurt a. M.: Fischer Taschenbuch 2002, S. 72-106.

Ders.: »Der Brief [1981]«, in: Ders., Erzählungen (2002), S. 107-179.

Hoffmann, Ernst Theodor Amadeus: Die Serapions-Brüder, hrsg. von Wulf Segebrecht, Frankfurt a.M.: Deutscher Klassiker-Verlag 2008.

Houellebecq, Michel: Les particules élémentaires, 2 è edition, Paris: Flammarion 1998.

Jirgl, Reinhard: »Das poetische Vermögen des alphanumerischen Codes in der Prosa«, in: Ders., Gewitterlicht. Erzählung, 5. Aufl., Hannover: Revonnah 2003, S. 50-77.

Ders.: Abschied von den Feinden [1995], München: dtv 1998.

Johnson, Uwe: Mutmaßungen über Jakob [1959], Frankfurt a.M.: Suhrkamp 1992.

Kermani, Navid: Dein Name [2011], 3. Aufl., Reinbek: Rowohlt 2015.

Ders.: Über den Zufall. Jean Paul, Hölderlin und der Roman, den ich schreibe. Frankfurter Poetikvorlesungen, München: Hanser 2012. 
Ders.: »Trostlose Normalität«, in: Neue Zürcher Zeitung. Zeitbilder vom 16.01.2007, S. $311 \mathrm{f}$.

Ders.: »Trostlose Normalität. Mit der NATO in Afghanistan«, in: Kursbuch 167 (2007), Heft 2, S. 1-19. Seitenangabe zit. nach https://www.yumpu.com/de/d ocument/read/33080701/trostlose-normalitat-navid-kermani vom 16.02.2021.

Kluge, Alexander: »8. Unheimlichkeit der Zeit. Neue Geschichten. Hefte 1-18« [1977], in: Ders., Chronik der Gefühle, Bd. II, Lebensläufe, Frankfurt a.M.: Suhrkamp 2004.

Ders.: »10. Lebensläufe« [1964], In: Ders., Chronik der Gefühle, Bd. II, Lebensläufe, Frankfurt a.M.: Suhrkamp 2004.

Ders.: Das fünfte Buch. Neue Lebensläufe. 402 Geschichten, Berlin: Suhrkamp 2012.

Ders.: Theorie der Erzählung. Frankfurter Poetikvorlesungen, Berlin: Suhrkamp 2013, Beiheft zur DVD.

Ders.: Kongs große Stunde. Chronik des Zusammenhangs, Berlin: Suhrkamp 2015.

Kühn, Dieter: N [1970], Neuausgabe, Frankfurt a.M.: S. Fischer 2005.

Morshäuser, Bodo: Die Berliner Simulation [1983], Berlin: Berliner Zeitung / Paperview 2007.

Musil, Robert: Der Mann ohne Eigenschaften [1930], 2 Bde, neu durchgesehene u. verbesserte Aufl., hrsg. von Adolf Frisé, Reinbek: Rowohlt 1981.

Negt, Oskar/Kluge, Alexander: Geschichte und Eigensinn. 7. Aufl., Frankfurt a.M.: Zweitausendeins 1983.

Proust, Marcel: Auf der Suche nach der verlorenen Zeit, Frankfurter Ausgabe, Bd.1: Unterwegs zu Swann, hrsg. von Luzius Keller, übers. aus d. Franz. von Eva Rechel-Mertens, revidiert von Luzius Keller, Frankfurt a.M.: Suhrkamp 1994.

Ders.: Auf der Suche nach der verlorenen Zeit, Frankfurter Ausgabe, Bd. 7: Die wiedergefundene Zeit, übers. aus d. Franz. von Eva Rechel-Mertens, revidiert von Luzius Keller. Frankfurt a.M.: Suhrkamp 1994.

Schmitt, Éric-Emanuel: Adolf H. Zwei Leben, übers. aus d. Franz. von Klaus Laabs, Zürich: Ammann 2007. 


\section{Sekundärliteratur}

Angehrn, Emil: Sein Leben schreiben. Wege der Erinnerung, Frankfurt a.M.: Klostermann 2017 (RoteReihe, Band 94).

Baudrillard, Jean: Der symbolische Tausch und der Tod [1976], übers. aus d. Franz. von Gerd Bergfleth et al, München: Matthes \& Seitz 1991 (=Batterien, Band 14).

Ders.: Agonie des Realen, übers. aus d. Franz. von Lothar Kurzawa und Volker Schaefer, Berlin: Merve 1978.

Beck, Ulrich: Risikogesellschaft. Auf dem Weg in eine andere Moderne, 21. Aufl., Frankfurt a.M.: Suhrkamp 2012.

Beuthan, Ralf: Das Undarstellbare. Film und Philosophie, Metaphysik und Moderne, Würzburg: Königshausen \& Neumann 2006 (=Film-Medium-Diskurs, Band 18).

Bobzin, Henning: Von Bremen in die Anderswelt. Über Identität und Realität in Prosahauptwerk, Poetik und Weblog von Alban Nikolai Herbst. Dissertation, Universität Göttingen 2015, http://ediss.uni-goettingen.de/bits tream/handle/11858/00-1735-0000-0022-600B-B/Bobzin2015-Dissertation.pdf? sequence $=3$ vom 28.06.19.

Böhme, Hartmut: Hubert Fichte. Riten des Autors und Leben der Literatur, Stuttgart: J.B. Metzler 1992.

Bürger, Peter: Theorie der Avantgarde, Frankfurt a.M.: Suhrkamp 1974.

Cramer, Friedrich: Chaos und Ordnung. Die komplexe Struktur des Lebendigen, 2. Aufl., Stuttgart: Deutsche Verlags-Anstalt 1989.

Dauner, Dorea: Literarische Selbstreflexivität. Dissertation, Universität Stuttgart 2009, http://dx.doi.org/10.18419/opus-5332 vom 19.05.20.

Dreyfus, Hubert/Taylor, Charles: Die Wiedergewinnung des Realismus, übers. aus d. Engl. von Joachim Schulte, Frankfurt a.M.: Suhrkamp 2016.

Eckholt, Matthias: Kann sich das Bewusstsein bewusst sein? Gespräche mit Dirk Baecker et al., Heidelberg: Carl-Auer 2017.

Emmerich, Wolfgang: Kleine Literaturgeschichte der DDR, erweiterte Neuausgabe, Berlin: Aufbau 2000.

Fauconnier, Gilles/Turner, Mark: The way we think. Conceptual blending and the minds hidden complexities, New York: Basic Books 2002.

Fischer-Lichte, Erika: Ästhetik des Performativen, Frankfurt a.M.: Suhrkamp 2004.

Flusser, Vilém: Die Schrift. Hat Schreiben Zukunft? Frankfurt a.M.: Fischer 1992. 
Foucault, Michel: Die Ordnung der Dinge. Eine Archäologie der Humanwissenschaften, 14. Aufl., übers. aus d. Franz. von Ulrich Köppen, Frankfurt a.M.: Suhrkamp 1997.

Frank, Manfred: Ansichten der Subjektivität, Berlin: Suhrkamp 2012.

Gabriel, Markus: Fiktionen, Berlin: Suhrkamp 2020.

Gadamer, Hans-Georg: Wahrheit und Methode. Grundzüge einer philosophischen Hermeneutik, 3. erweit. Aufl., Tübingen: Mohr-Siebeck 1960.

Genette, Gérard: Palimpseste. Die Literatur auf zweiter Stufe, übers. aus d. Franz. von Wolfram Bayer und Dieter Hornig, Frankfurt a.M.: Suhrkamp 1993.

Gossens, Peter: »NNeue Weltliteratur ? Goethes Weltliteratur-Begriff im Kontext der Globalisierung «, in: Goethe-Jahrbuch, hrsg. von Frieder von Ammon, Jochen Golz, Edith Zehm, 134. Band, Göttingen: Wallstein 2017, S. 39-46.

Habermas, Jürgen/Luhmann, Niklas (Hg.): Theorie der Gesellschaft oder Sozialtechnologie - Was leistet die Systemforschung?, Frankfurt a.M.: Suhrkamp 1971.

Hiergeist, Teresa: Erlesene Erlebnisse. Formen der Partizipation an narrativen Texten, Bielefeld: transcript 2014 (=Lettre).

Hofmann, Michael/v. Stosch, Klaus/Schulte Eickholt, Swen: Navid Kermani, Würzburg: Königshausen \& Neumann 2019 (=Profile der Gegenwartsliteratur, Band 1-2019).

Holdenried, Michaela: Autobiographie, Stuttgart: Reclam 2000.

Igl, Natalia: »Romantische Rahmen-Binnen-Konstruktionen als >Mapping von inner- und außertextuellen Räumen«, in: Mikuláš/Wege (Hg.), Schlüsselkonzepte (2016), S. 81-100.

Iser, Wolfgang: Das Fiktive und das Imaginäre: Perspektiven literarischer Anthropologie, Frankfurt a.M.: Suhrkamp 1991.

Ders.: Emergenz. Nachgelassene und verstreut publizierte Essays, hrsg. von Alexander Schmitz, Konstanz: University Press 2013.

Jürgensen, Christoph: »Ins Netz gegangen - Inszenierungen von Autorschaft im Internet am Beispiel von Rainald Goetz und Alban Nikolai Herbst«, in: Christoph Jürgensen/Gerhard Kaiser (Hg.), Schriftstellerische Inszenierungspraktiken - Typologie und Geschichte, Heidelberg: Winter 2011 (=Beihefte zum Euphorion, Band 62), S. 403-422.

Klimek, Sonja: Paradoxes Erzählen. Die Metalepse in der phantastischen Literatur, Paderborn: Mentis 2010 (=Explicatio). 
Klinkert, Thomas: Epistemologische Fiktionen. Zur Interferenz von Literatur und Wissenschaft seit der Aufklärung, Berlin: De Gruyter 2010 (Linguae \& litterae, Band 2).

Koschorke, Albrecht: Wahrheit und Erfindung. Grundzüge einer Allgemeinen Erzähltheorie, 2. Aufl., Frankfurt a.M.: S. Fischer 2012.

Ders. (Hg.): Komplexität und Einfachheit. DFG-Symposion 2015, Stuttgart: J.B. Metzler 2017.

Kosub, Sven: »Textuelle Berechenbarkeit«, in: Koschorke (Hg.): Komplexität und Einfachheit (2017), S. 240-255.

Kreknin, Innokentij: Poetiken des Selbst. Identität, Autorschaft und Autofiktion am Beispiel von Rainald Goetz, Joachim Lottmann und Alban Nikolai Herbst. Berlin et al.: De Gruyter 2014 (=Studien zur deutschen Literatur, Band 206).

Krohn, Wolfgang/Küppers, Günter (Hg.): Emergenz: Die Entstehung von Ordnung, Organisation und Bedeutung, Frankfurt a.M.: Suhrkamp 1992.

Largier, Niklaus: »Sektion 1: Praktiken der Einfachheit. Einführung«, in: Koschorke (Hg.), Komplexität und Einfachheit (2017), S. 11-18.

Linke, Detlef B: Die Freiheit und das Gehirn. Eine neurophilosophische Ethik. München: C.H. Beck 2005.

Lommel, Michael: Im Wartesaal der Möglichkeiten. Lebensvarianten in der Postmoderne, Köln: Halem 2011.

Lukács, Georg: Die Theorie des Romans. Ein geschichtsphilosophischer Versuch über die Formen der großen Epik [1920], 3. Aufl., Darmstadt et al.: Luchterhand 1976.

Mainzer, Klaus: Komplexität, Paderborn: W. Fink 2008 (UTB Profile, Band 3012).

Martinez, Matias/Scheffel, Michael: Einführung in die Erzähltheorie [1999]. 11. überarb. u. aktual. Aufl., München: C.H. Beck 2020 (=C.H. Beck Studium).

McLuhan, Marshall: Die magischen Kanäle. Understanding Media, 2. erweit. Aufl., hrsg. von Gerti Fietzek und Michael Glasmeier, Dresden et al.: Verlag der Kunst 1995.

Meuter, Norbert: Narrative Identität. Das Problem der personalen Identität im Anschluß an Ernst Tugendhat, Niklas Luhmann und Paul Ricœur, Stuttgart: M \& P Verlag 1995.

Mieth, Dietmar: Moral und Erfahrung I. Grundlagen einer theologisch-ethischen Hermeneutik, 4. überarb. und ergänz. Neuaufl., Fribourg: Universitätsverlag 1999 (=Studien zur theologischen Ethik, Band 2). 
Mikuláš, Roman/Wege, Sophia (Hg.): Schlüsselkonzepte und Anwendungen der Kognitiven Literaturwissenschaft, Münster: Mentis 2016 (=Poetogenesis, Studien und Texte zur empirischen Anthropologie der Literatur, Band 11).

Nassehi, Armin: Muster. Theorie der digitalen Gesellschaft, München: C.H. Beck 2019.

Ders.: Die letzte Stunde der Wahrheit. Kritik der komplexitätsvergessenen Vernunft, Hamburg: Sven Murmann 2017.

Niehaus, Michael: »Das bessere Argument. Eine Anmerkung zur Logik des Argumentierens bei Jürgen Habermas«, in: Philosophisches Jahrbuch 105 (1998) H. II., S. 412-422.

Parfit, Derek: Reasons and Persons, Oxford: University Press 1986.

Reckwitz, Andreas: Subjekt, Bielefeld: transcript 2008 (=Einsichten. Themen der Soziologie).

Richter, Sandra: Eine Weltgeschichte der deutschsprachigen Literatur, München: C. Bertelsmann 2017.

Ricœur, Paul: Zeit und Erzählung, Bd. 1: Zeit und historische Erzählung, übers. aus d. Franz. von Rainer Rochlitz, München: W. Fink 1988 (=Übergänge, Band 18/1).

Rodiek, Christoph: Untersuchungen zur spanischen Kurzgeschichte der Gegenwart (1980-2010). Definitionen, Analysen, Fallstudien, Frankfurt a.M.: Peter Lang 2012.

Roth, Gerhard: »Kognition: Die Entstehung von Bedeutung im Gehirn«, in: Krohn/Küppers (Hg.), Emergenz (1992), S. 104-133.

Ders.: Das Gehirn und seine Wirklichkeit. Kognitive Neurobiologie und ihre philosophischen Konsequenzen, Frankfurt a.M.: Suhrkamp 1997.

Sander, Ulrike-Christine: Ichverlust und fiktionaler Selbstentwurf. Die Romane Lars Gustafssons, Göttingen: Vandenhoeck \& Ruprecht 1998 (=Palaestra. Untersuchungen aus der deutschen und skandinavischen Philologie, Band 305).

Scheffel, Michael: Formen selbstreflexiven Erzählens. Eine Typologie und sechs exemplarische Analysen, Tübingen: Niemeyer 1997 (=Studien zur deutschen Literatur, Band 145).

Scheffer, Bernd: Interpretation und Lebensroman. $\mathrm{Zu}$ einer konstruktivistischen Literaturtheorie, Frankfurt a.M.: Suhrkamp 1992.

Scheerer, Thomas M.: Mario Vargas Llosa - Leben und Werk. Eine Einführung, Frankfurt a.M.: Suhrkamp 1991. 
Schmidt, Siegfried J.: Grundriß der Empirischen Literaturwissenschaft, Frankfurt a.M.: Suhrkamp 1991.

Ders.: »Über die Rolle von Selbstorganisation beim Sprachverstehen«, in: Krohn/Küppers (Hg.), Emergenz (1992), S. 293-333.

Schulte, Joachim: Wittgenstein. Eine Einführung, Stuttgart: Reclam, 2009.

Schulte Eickholt, Swen: »Dein Name«, in: Hofmann/v. Stosch/Schulte Eickholt, Navid Kermani (2019) S. 103-130.

Ders.: »Über den Zufall. Jean Paul, Hölderlin und der Roman, den ich schreibe«, in: Hofmann/v. Stosch/Schulte Eickholt, Navid Kermani (2019), S. $131-150$

Sistig, Sabine: Wandel der Ich-Identität in der Postmoderne. Zeit und Erzählen in Wolfgang Hilbigs »Ich« und Peter Kurzecks »Keiner stirbt«, Würzburg: Königshausen \& Neumann 2003 (=Epistemata. Reihe Literaturwissenschaft, Band 407).

Sloterdijk, Peter: Du mußt dein Leben ändern. Über Anthropotechnik, Frankfurt a.M.: Suhrkamp 2012.

Ders.: Zur Welt kommen - Zur Sprache kommen. Frankfurter Vorlesungen, Frankfurt a.M.: Suhrkamp 1988.

Steiner, André: Das narrative Selbst - Studien zum Erzählwerk Wolfgang Hilbigs. Erzählungen 1979-1991, Romane 1989-2000, Frankfurt a.M. et al.: Peter Lang 2008 (=Europäische Hochschulschriften. Reihe 1, Deutsche Sprache und Literatur, Band 1970).

Ders.: »Filmisch inszenierte Simultaneität als Mittel der Darstellung nichtlinearer Szenarios«, in: Philipp Hubmann/Till Julian Huss (Hg.), Simultaneität. Modelle der Gleichzeitigkeit in den Wissenschaften und Künsten, Bielefeld: transcript 2013, S. 381-399.

Ders.: »Eine andere Gegenwart? - Annäherungen zwischen Erzähltheorie und Neurowissenschaft «, in: Revista de Filologia Alemana 22(2014), S. 11-29.

Ders.: "Zyprian oder Zombie?« - Zur Bedeutung der Rezeption der `Serapionsbrüder E.T.A. Hoffmanns für die Genealogie des Erzählens im Roman >Eine Übertragung «, in: Norman Kasper/Gert Theile (Hg.), Asozialität und Aura. Wolfgang Hilbig und die Romantik, Leiden et al.: W. Fink 2017, S. 71-88.

Varela, Francisco J.: Kognitionswissenschaft - Kognitionstechnik. Eine Skizze aktueller Perspektiven, 2. Aufl., übers. aus d. Engl. von Wolfram Karl Köck, Frankfurt a.M.: Suhrkamp 1990. 
v. Foerster, Heinz: »Verstehen verstehen«, in: Ders., Wissen und Gewissen. Versuch einer Brücke, hrsg. von Siegfried J. Schmidt, übers. aus d. Amerik. von Wolfram Karl Köck, Frankfurt a.M.: Suhrkamp 1993.

Virilio, Paul: Fluchtgeschwindigkeit, 2. Aufl., übers. aus d. Franz. von Bernd Wilczek, Frankfurt a.M.: Fischer Taschenbuch 2001.

v. Werder, Lutz: Lehrbuch des kreativen Schreibens, Wiesbaden: Marix 2007. Wagner-Egelhaaf, Martina: Autobiographie, 2. aktual. und erweit. Aufl., Stuttgart: Metzler 2005.

Wege, Sophia: »Die kognitive Literaturwissenschaft lässt sich blenden. Anmerkungen zum Emergenz-Begriff der Blending-Theorie«, in: Mikuláš/Wege (Hg.), Schlüsselkonzepte (2016), S. 243-260.

Welzer, Harald et al.: »Opa war kein Nazi«. Nationalsozialismus und Holocaust im Familiengedächtnis, 9. Aufl., Frankfurt a.M.: Fischer Taschenbuch 2015.

Welsch, Wolfgang: »Was ist eigentlich Transkulturalität?«, in: Dorothee Kimmich/Schamma Schahadat (Hg.), Kulturen in Bewegung. Beiträge zur Theorie und Praxis der Transkulturalität, Bielefeld: transcript 2012 (=Kultur- und Medientheorie), S. 25-40.

Wrobel, Dieter: Postmodernes Chaos - Chaotische Postmoderne. Eine Studie zu Analogien zwischen Chaostheorie und deutschsprachiger Prosa der Postmoderne, Bielefeld: Aisthesis 1997.

Zink, Dominik: Interkulturelles Gedächtnis. Ostwestliche Transfers bei Saša Stanišić, Nino Haratischwili, Julya Rabinowich, Richard Wagner, Aglaja Veteranyi und Herta Müller, Würzburg: Königshausen \& Neumann 2017(=Epistemata. Reihe Literaturwissenschaft, Band 865).

Zipfel, Frank: »Autofiktion«, in: Dieter Lamping (Hg.), Handbuch der Literarischen Gattungen, Stuttgart: Kröner 2009, S. 31-36.

Ders.: »Autofiktion. Zwischen den Grenzen von Faktualität, Fiktionalität und Literarität?«, in: Simone Winko et al. (Hg.), Grenzen der Literatur. Zu Begriff und Phänomen des Literarischen, Berlin et al.: De Gruyter 2009 (=Revisionen, Band 2), S. 283-314. 


\section{Nachschlagewerke}

Bußmann, Hadumod: Lexikon der Sprachwissenschaft, 2. völlig neu bearb. Aufl., Stuttgart: Kröner 1990.

Der Brockhaus Literatur: Schriftsteller, Werke, Epochen, Sachbegriffe, 2. völlig neu bearb. Aufl., hrsg. von der Lexikonredaktion des Verlags F.A. Brockhaus, Leipzig et al.: Brockhaus 2004.

Krause, Detlev: Luhmann-Lexikon. Eine Einführung in das Gesamtwerk von Niklas Luhmann, 2. vollständig überarb., erweit. und aktual. Aufl., Stuttgart: Enke Verlag 1999.

Nünning, Ansgar (Hg.): Metzler Lexikon Literatur- und Kulturtheorie. Ansätze - Personen - Grundbegriffe, 2. überarb. u. erweit. Aufl., Stuttgart et al.: J.B. Metzler 2001.

WikipediA. Die freie Enzyklopädie: Lemma »Feigenbaum-Konstante«, https:/ /de.wikipedia.org/wiki/Feigenbaum-Konstante vom 16.02.2021.

Ebd.: Lemma »Träges Wissen«, https://de.wikipedia.org/wiki/Tr\%C3\%A4ges Wis sen vom 16.02.2021

Ebd.: Lemma »Quantengravitation«, https://de.wikipedia.org/wiki/Quantengrav itation vom 06.08.2020.

Ebd.: Lemma »Klaus-Jürgen Rattay«, https://de.wikipedia.org/wiki/Klaus-J\%C3\% BCrgen_Rattay vom 30.10.2020.

Ebd.: Lemma »Anthropozän«, https://de.wikipedia.org/wiki/Anthropoz\%C3\%A4n vom 27.12.2020.

\section{Filmografie}

INCEPTION (USA/GB 2010, R: Christopher Nolan)

MATRIX (USA/AUS 1999, R: Lana und Lilly Wachowski)

Die Patriotin (BRD 1979, R: Alexander Kluge)

Vantage Point, dt. 8 Blickwinkel (USA 2007, R: Pete Travis)

WELT AM DRAHT (BRD 1973, R: Rainer Werner Fassbinder) 


\section{Radio- und Fernsehsendungen}

ESSAY UND DiskURS: »Saisonschluss (3/3)«, von Mathias Greffrath, DLF, Sendung vom 22.12.2019. Text online abrufbar unter https://www.deutsc hlandfunk.de/rueckblick-und-ausblick-saisonschluss-3-3.1184.de.html?d ram:article_id=464820 vom 17.05.2020.

SCOBEL: »Systeme auf der Kippe«, 3sat, Sendung vom 10.12.2020, mit Gert Scobel. Gäste: Patrizia Nanz, Timo Goeschl, Stefan Thurner.

SCOBEL: »Ethik fürs Digitale«, 3sat, Sendung vom 03.09.2020, mit Gert Scobel. Gäste: Markus Gabriel, Yvonne Hofstetter, Armin Nassehi.

STUdio LCB, Deutschlandradio Kultur, Sendung mit Navid Kermani vom 27. August 2011, Zitat Andreas Isenschmid. Textauszug online abrufbar unter https:/www.navidkermani.de/dein-name/pressestimmen/vo m 17.06.2020. 


\section{Sachregister}

A

abarischer Punkt, 149

alternative Chronologien, 54, 187

Analogie, 15, 109, 117, 120, 124, 142, $149,179,190$

Aspektsehen, 28

ästhetische Komplexität, 33, 35

Attraktor, 41, 160, 169

autofiktionales Erzählen, 47, 92, 97, 99

Autonomie, 30, 31, 128, 135, 136, 194

Autopoiese, 67, 139

autopoietisch, 13, 69, 94, 153, 154

\section{B}

Beobachtung dritter Ordnung, 29

Beobachtung zweiter Ordnung, 29, 37

Beobachtungskaskade, 36, 183

Berechenbarkeit, 84

Bezugssystem, 8, 25, 30, 86, 115, $139,142,169$

Bifurkation, 15, 40, 147

Blending, 14, 82, 83, 94, 113

C

Chaos, 36, 40, 88, 115, 149

Chaostheorie, 14, 15, 37, 179

Clash, 36, 37, 70, 192
Climate fiction, 42

Closed circuit, 189

Creative writing, 112

D

Dekonstruktion, 25, 136

Dezentrierung des Subjekts, 70, 71

Differance, 77, 84

doppelte Konditionierung, 76

E

Echtzeit, 91, 93-96, 105-107, 109, 111, 112, 130, 134, 167, 190, 191

Echtzeitroman, 91

Effet de réel, 80, 106

Embedded knowledge, 76, 77

Emergenz, 17, 43, 52, 56, 59, 60, 63, $68,81,97,101,102,109$, 111, 139, 150, 154, 189, 191

emotionale Partizipation, 77

empirischer Autor, 25, 175

Endhorizont der Rezeption, 68, 103

endlos autobiographische Tätigkeit der Wahrnehmung, 83, 133, 162

Erinnerungskaskade, 61, 185

Erlebnismodell, 55, 190, 191 
$\mathbf{F}$

Fact, 97

Fake, 97

Familiengedächtnis, 152

Fehlen einer zentralen Instanz, 27, 39, 156

Fluktuation, 40

fraktal, 71, 100, 169, 179, 182, 194

Fraktale, 15, 181

fraktales Erzählen, 125, 186

fundamentale Komplexität, 116-

120

\section{G \\ generativer Einschnitt, 52, 150 \\ globale Klimaerwärmung, 149 \\ Globalisierung, 85, 86}

\section{$\mathbf{H}$}

Herausforderung durch Komplexität, 14, 28, 41, 42

Hermeneutik, 77, 78, 86, 88

hermeneutisch, 72, 75, 86, 88, 190

heteroreferenziell, 141

heteroreferenzielles Erzählen, 140, 154

Histoire croisée, 86

Homunkulus-Problem, 125, 183, 184, 186

Homunkulus-Struktur, 184, 186, 188, 189

\section{I}

Identität, 9, 14, 19, 20, 22, 24, 28, $35,44,45,47-49,51-53$, $57,61,64-66,69,71,85$, $92,99,107,114,129,132$, 136, 139-142, 146, 155,
$156,163,167,171,177$, $180,182,187,191,192$

Identitätsdiffusion, 180

Inert knowledge, 77

interkulturelles Gedächtnis, 89

Intervall, 91, 93

Iteration, 29, 109, 192

iterativ, 40, 69, 110, 186

K

Kaskade von Möglichkeiten, 53, 143, 150, 154, 181

Kippfigur, 29

Klimakatastrophe, 42

Klimawandel, 42

Kognition, 75, 116, 153

Kognitionsforschung, 57, 83

kognitiv, 13, 36, 38, 44, 79, 119, 126, 134, 188

kognitive Erkenntnis, 81

kognitive Interaktion, 152

kognitive Literaturwissenschaft, 17, 83,128

kognitive Operation, 57, 72

kognitive Partizipation, 79

kognitive Psychologie, 113

kognitive Repräsentation, 82

kognitiver Prozess, 15, 65, 83, 117

kognitives System, 16, 17, 57, 65, $67,70,75,104,119,186$

kognitives Verstehen, 72

Kohärenz, 14, 36, 38, 71, 141, 146, 180

Kokonisierung, 126

komplex rückgekoppelte Beobachtung, 25, 28, 35, 110

komplexe Metalepse, 48, 182 
komplexes Erzählen, 7, 10, 20, 26,

$33,34,41,63,69,113$, 118,186

Komplexität, 9, 10, 13, 15, 19, 21, $23,28-30,33,39,41,42$, $72,75,79-82,86-88$, 91, 94, 98, 100, 103, 106, $108,112,115,118-120$, $123,125,126,129,132$, 140, 144-146, 157, 159, $160,175,180,186,187$, 189, 191, 193

Komplexitätsforschung, 7, 14, 17, $41,42,84,107,119,145$

Komponente, 17, 22, 28, 52, 67-70, $72,81,94,103,104,108$, $111,118,147,150,159$, $169,181,185,186$

Konnektom, 118

Kontakttheorie der Wirklichkeit, 125

Kontinuität, 14, 48, 141, 143, 146, 180

Konvergenzzentrum, 55, 155

Kreativitätsforschung, 17

kybernetisch, 20, 22, 30, 35, 63, 64, $71,170,173,190,191$

kybernetische Rückkopplung, 26, $56,59,148,160$

Kybernetischer Realismus, 25, 27, $44,66,72,116$

\section{L}

Lebensvarianten, 107, 142, 192 literarische Komplexität, 17, 84 Literatur auf zwei plus n-ter Stufe, 10
M

Matrix, 22, 67, 69, 72, 84, 111, 173

mediale Repräsentation, 113

Mehrfacherzählung, 179, 180

Mehrfachkodierung, 16, 28, 29, 76, 191

Mehrstelligkeit, 52, 59, 63, 68, 150, 186

mentale Repräsentation, 14, 75, 79, $83,93,124,127,129$

Mereologie, 88

mereologisch, 88, 170

metaleptisch, 26, 29, 35, 51, 53, 63, $83,92,183$

Mise en abyme, 52, 164, 194

Möbiusband, 189

Möbiusband-Erzählung, 48, 51, 64, 182

Modelle der Simulation, 55, 103, $130,159,160,164,190$

$\mathbf{N}$

negative Rückkopplung, 102

Netzwerk, 27, 29, 40, 64, 120, 128, $136,164,194$

netzwerkartig, 170, 190, 191, 194, 195

nicht-lineare Dynamik, 145, 150

Nullstelle des Diskurses, 51, 65, 139, 163, 177

$\mathbf{P}$

Paradox der verschwundenen Welt, 125,183

Parodie, 31, 129, 167, 188

parodistisch, 151, 166, 167, 187, 191

Perspektivendifferenz, 8, 17, 22, 34,108 
Perspektivenwechsel, 35

Polykontexturalität, 28

polyphones Erzählen, 15

positive Rückkopplung, 101

Programmierung, 19, 64, 110, 173

\section{$\mathbf{R}$}

Randbedingung, 14, 26, 35, 40, 43, $83,112,116$

recyceln, 160

Recycling, 101

Reduktion, 34, 39, 43, 106, 108, $116,127,140,157,160$, 190, 192

Regelkreis, 25, 64, 80, 143

Rekombination, 159, 163

Rekursion, 61, 109, 111, 112, 135, $136,184,195$

Rekursivität, 52, 109, 140

Reversibilität der Sprecherperspektive, 183

Rezeption, 14-16, 19, 38, 39, 41, $43,55,57,62,67,68,71$, $72,77,78,81,82,84,97$, 104, 106, 114, 116-118, $126,128,130,131,134$, $152,153,171,186,189$, 192

Rezeptionsästhetik, 17

rezeptionsästhetisch, 29

rezeptionstheoretisch, 37,79

Risikogesellschaft, 135

Rückkopplung, 40, 52, 53, 56, 59, $63,65,109,132,135,139$, 140, 144, 149, 154, 170, 175

Rückkopplungseffekt, 193
Rückkopplungsschleife, 101, 109, 111

rückläufige Kausalität, 52, 64, 65, 150

Rückübersetzung, 189-191

S

Selbstähnlichkeit, 69, 169

Selbstermächtigung, 30, 31

Selbstorganisation, 57, 110

selbstreferenziell, 13, 57, 139, 142, 154

selbstreferenzielle Operation, 57, 65,67

selbstreferenzielle Prozesse, 83 selbstreferenzielle Verdoppelung, 186

selbstreferenzieller Zirkel, 70, 184 selbstreferenzielles Erzählen, 140 selbstreflexiv, 13

Selbstreflexivität, 13

Selbstwirksamkeit, 42, 132, 144, 193

Selektion, 104, 163

seltsame Schleife, 109

Shift, 23, 25

Simulakrum, 159, 163, 188

Simulation, 21, 22, 25, 31, 55, 63, $93,98,103,119,130,142$, $155,156,160-166,168$, 173, 187-189, 191, 193, 194

simultan, 16, 22, 35, 39, 54, 64, 65, $116,131,141,169,195$

Simultaneität, 81, 190

Situated learning, 77

Strategie von unten, 147

Switch, 100 


\section{$\mathbf{T}$}

totaler Roman, 44, 110

Trajektorie, 93

Transkulturalität, 85, 91

transkulturell, 75, 87, 89

Transkulturelle Germanistik, 87

transkulturelle Kommunikation, 190

\section{U}

Übersetzung, 30, 191

Übersetzungskonflikt, 29

Übersetzungsproblem, 193

Übersetzungsstrategie, 31, 128, 195

Uminterpretation, 35, 72, 136

unendliche Semiose, 77, 84

Universalie, 59

Unlogische Heterarchie, 182

Unschärferelation, 53, 117, 120, 186

Unvorhersehbarkeit, 42, 118, 145

\section{V}

Verhaltensänderung, 47, 111, 136

veritative Symmetrie, 183

verkörperter Akteur, 124

vermittlungsgebunden, 124

vermittlungsgebundene Auffassung, 127, 129

Vermittlungsgebundenheit, 125

Versprachlichung, 9, 108, 112, 115, 116, 175

verwickelte Hierarchie, 182

virtuell, 8, 69, 92, 129, 193

virtuelle Realität, 64, 111, 193, 194

W

Wicked problems, 186, 193, 195

\section{$\mathbf{Z}$}

Zeitumkehr, 54, 93, 190

Zufall, 9, 68, 103, 105, 108, 112, 118,

$157,180,184$ 


\section{Literaturwissenschaft}

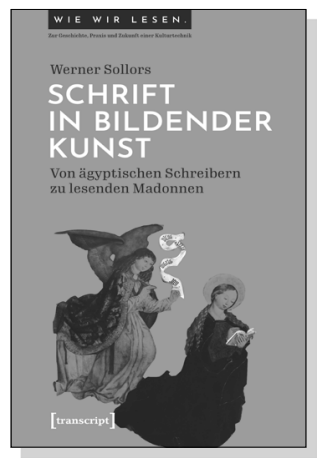

Werner Sollors

\section{Schrift in bildender Kunst}

Von ägyptischen Schreibern zu lesenden Madonnen

September 2020, 150 S., kart.,

14 Farbabbildungen, 5 SW-Abbildungen

16,50€ (DE), 978-3-8376-5298-7

E-Book:

PDF: $14,99 €$ (DE), ISBN 978-3-8394-5298-1

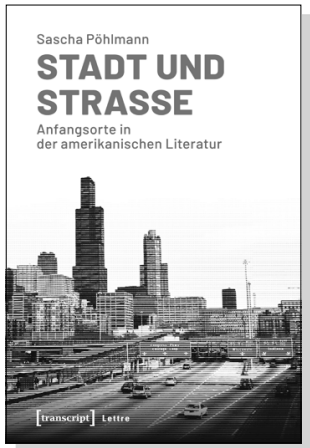

Sascha Pöhlmann

\section{Stadt und Straße}

Anfangsorte in der amerikanischen Literatur

2018, 266 S., kart.

29,99€ (DE), 978-3-8376-4402-9

E-Book:

PDF: $26,99 €$ (DE), ISBN 978-3-8394-4402-3

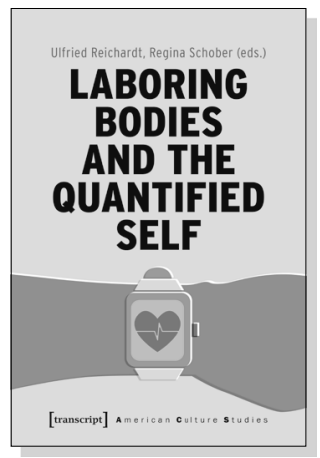

Ulfried Reichardt, Regina Schober (eds.)

Laboring Bodies and the Quantified Self

October 2020, 246 p., pb.

40,00€ (DE), 978-3-8376-4921-5

E-Book:

PDF: 39,99 € (DE), ISBN 978-3-8394-4921-9 


\section{Literaturwissenschaft}
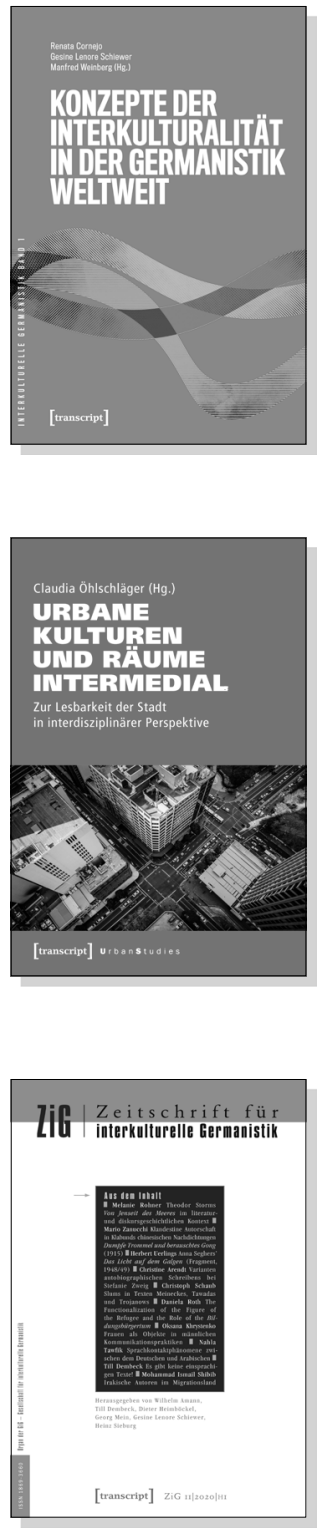

Renata Cornejo, Gesine Lenore Schiewer, Manfred Weinberg (Hg.)

Konzepte der Interkulturalität in der Germanistik weltweit

August 2020, 432 S., kart., 6 SW-Abbildungen 50,00€ (DE), 978-3-8376-5041-9

E-Book: kostenlos erhältlich als Open-Access-Publikation PDF: ISBN 978-3-8394-5041-3

Claudia Öhlschläger (Hg.)

\section{Urbane Kulturen und Räume intermedial}

Zur Lesbarkeit der Stadt in interdisziplinärer Perspektive

Juli 2020, 258 S., kart., 10 SW-Abbildungen

40,00€ (DE), 978-3-8376-4884-3

E-Book:

PDF: 39,99€ (DE), ISBN 978-3-8394-4884-7

Wilhelm Amann, Till Dembeck, Dieter Heimböckel, Georg Mein, Gesine Lenore Schiewer, Heinz Sieburg (Hg.) Zeitschrift für interkulturelle Germanistik 11. Jahrgang, 2020, Heft 1

August 2020, 226 S., kart.

$12,80 €(D E), 978-3-8376-4944-4$

E-Book:

PDF: $12,80 €(D E)$, ISBN 978-3-8394-4944-8 
